\title{
ESTIMATION AND CORRECTION OF GEOMETRIC \\ DISTORTIONS IN SIDE-SCAN SONAR IMAGES
}

by

\section{Daniel TÁvora dé QueIROZ Cobra}

S.B., Universidade de Brasília, Brazil (1983)

S.M., Massachusetts Institute of Technology (1986)

Submitted in partial fulfillment of the requirements for the degree of

DOCTOR OF PHILOSOPHY

at the

MassachusetTs Institute of TechNology

and the

WOODS HOLE OCEANOGRAPHIC INSTITUTION

March 1990

(C) Massachusetts Institute of Technology and

Woods Hole Oceanographic Institution, 1990

All rights reserved.

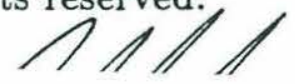

\begin{tabular}{|c|}
\hline $\begin{array}{c}\text { MARINE } \\
\text { BIOLOGICAL } \\
\text { LABORATORY }\end{array}$ \\
\hline LIBRARY \\
\hline $\begin{array}{l}\text { WOODS HOLE, MASS. } \\
\text { W. H. O. I. }\end{array}$ \\
\hline
\end{tabular}

Signature of Author

Joint Program in Oceanography and Oceanographic Engineering

Massachusetts Institute of Technology / Woods Hole Oceanographic Institution

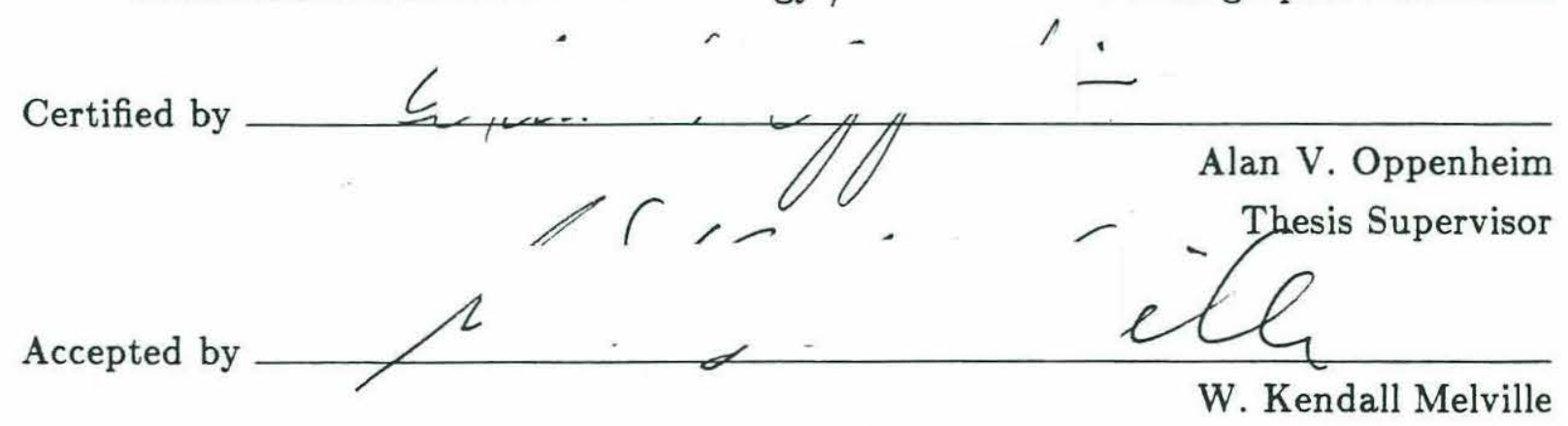

Chairman, Joint Committee for Ocean Engineering Massachusetts Institute of Technology / Woods Hole Oceanographic Institution

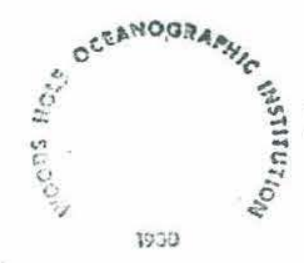




\title{
ESTIMATION AND CORRECTION OF GEOMETRIC \\ DISTORTIONS IN SIDE-SCAN SONAR IMAGES
}

\author{
by \\ Daniel Távora de Queiroz Cobra
}

\begin{abstract}
Submitted to the Joint Program in Oceanography and Oceanographic Engineering of the Massachusetts Institute of Technology and the Woods Hole Oceanographic Institution on March 30, 1990 in partial fulfillment of the requirements for the Degree of Doctor of Philosophy in Electrical and Oceanographic Engineering.
\end{abstract}

\begin{abstract}
This thesis introduces a new procedure for the enhancement of acoustic images of the bottom of the sea produced by side-scan sonars. Specifically, it addresses the problem of estimating and correcting geometric distortions frequently observed in such images as a consequence of motion instabilities of the sonar array. This procedure estimates the geometric distortions from the image itself, without requiring any navigational or attitude measurements. A mathematical model for the distortions is derived from the geometry of the problem, and is applied to estimates of the local degree of geometric distortion obtained by cross-correlating segments of adjacent lines of the image. The model parameters are then recursively estimated through deterministic least-squares estimation. An alternative approach based on adaptive Kalman filtering is also proposed, providing a natural framework in which a priori information about the array dynamics may be easily incorporated. The estimates of the parameters of the distortion model are used to rectify the image, and may also be used for estimating the attitude parameters of the array. A simulation is employed to evaluate the effectiveness of this technique and examples of its application to high-resolution side-scan sonar images are provided.
\end{abstract}

Thesis Supervisor: Alan V. Oppenheim

Title: Professor of Electrical Engineering

This work was produced under sponsorship of the Conselho Nacional de Desenvolvimento Científico e Tecnológico ( $C N P q$ ), an agency of the Government of the Federative Republic of Brazil, and was supported in part by the Defense Advanced Research Projects Agency monitored by the Office of Naval Research under Grant No. N00014-89-J-1489, in part by the National Science Foundation under Grant No. MIP 87-14969, and in part by Sanders Associates, Incorporated. 
To my parents, Rubem and Maria José. 


\section{ACKNOWLEDGMENTS}

My participation in the MIT/WHOI Joint Program and this thesis itself are a direct consequence of an invitation extended to me by Prof. Alan Oppenheim to work on the application of signal processing techniques to side-scan sonar data. Working with Al has been a privilege and a very rewarding experience. I am indebted to him for all the guidance and support he has offered me as my thesis supervisor. Dr. Jules Jaffe was my supervisor at WHOI during the initial stages of the development of this thesis and helped determine the direction of my investigations. He also had an important role in the acquisition of our data set. I would also like to thank Prof. Bruce Musicus and Dr. W. Kenneth Stewart, Jr., for being on my thesis committee and for their many helpful comments. Bruce had also supervised my previous graduate work at MIT and I am grateful for the support and personal concern he showed during the less blissful moments of my graduate student experience. Many thanks also to Visiting Prof. Sidney Burrus, chairman of my thesis defense.

Students of the Digital Signal Processing Group will often state in their theses that working in DSPG constitutes a challenging and intellectually stimulating enterprise. I would add that it has also been a privilege to belong to this distinguished group of researchers. I will remember as part of my life at MIT the interaction with many of the students in the group, and in particular with Greg Wornell, my officemate, and Michele Covell and Tae Joo, with whom I had a friendly competition to see who would graduate first (they did). My thanks go to Giovanni Aliberti and Giampiero Sciutto, our system managers, for keeping our computer facility in excellent shape at all times, as well as for helping me brush up on my Italian. I also thank our administrative assistants, Deborah Gage and Cindy LeBlanc, for all their help throughout my years at DSPG. Deborah and Cindy helped create an enjoyable atmosphere at DSPG through many concrete gestures, such as the home-baked breads, cakes, and other treats they often brought us. Marilyn Pierce and the staff of the EECS Graduate Office were always very helpful and did a great job at tactfully dissuading me from becoming a tenured graduate student.

The best part of being in the MIT/WHOI joint program, of course, was working at Woods Hole during the summer. The Department of Ocean Engineering, my home at WHOI, provided a stimulating working environment. During my first two summers at WHOI I worked closely with two of Jules Jaffe's graduate students, U.S. Navy Lieuts. Jack Nicholson and John Richardson. They had an important role in the acquisition of the data used in this thesis. I would like to thank A. D. Colburn, Jr., skipper of the R. V. Asterias for skillfully taking us through the waters of the Vineyard Sound on the cruise during which we collected the data. I am also indebted to several engineers at WHOI for lending me the equipment I used to build the system for digitizing the sonar images. Many thanks also to the staff of the Ocean Engineering Department, the Joint Program and Education Offices, as well as the Housing Office and all others who helped make working at WHOI a rewarding and enjoyable experience.

I owe my first hands-on experience with sonar to the late Prof. Harold "Doc" Edgerton through the short course on sonar he taught every winter at MIT. Pinging the bottom of Boston Harbor with Doc in a freezing January day was an experience I will not soon 
forget. He also helped me establish helpful contacts at EG\&G, Inc., of Waltham, Mass., and at Klein Associates, Inc., of Salem, NH, both manufacturers of side-scan sonars. Part of the equipment used for acquiring our data was lent to us by the United States Geological Survey facility at Cape Cod, and Klein Associates lent us one of their $500 \mathrm{kHz}$ high-resolution towfish. I would like to thank Martin Klein, president of Klein Associates, for all his support and for his interest in the development of our work.

During most of my graduate program at MIT, I was supported by a scholarship from the Brazilian Government, through its Conselho Nacional de Desenvolvimento Científico e Tecnológico $(\mathrm{CNP} q)$. The facilities used at MIT were maintained in part by grants from the National Science Foundation and the Office of Naval Research of the U.S. Navy. I thank these institutions for their support. I am also grateful to the Advanced Television Research Project at MIT for the use of their facilities for producing the pictures in this thesis, and to several graduate students in that group for their help.

Throughout my studies at MIT I was backed up by the love, support and encouragement of my family in Brazil, and, more recently, also in Boston, where my sister Eveline now attends graduate school. My parents, Rubem and Maria José, encouraged me from an early age to strive for academic excellence, setting me on the path that eventually led to my graduate studies at MIT. One of the most significant aspects of my experience here was the realization that there are other equally important, and ultimately even more important, goals to pursue in life. I am grateful to several friends who helped me come to that realization, especially Fr. Bernard Campbell, Hugh Moran, Fritz Züger, Bill Neu, Siegfried Hitzler, Julian Ciabattini, my E-mail pal Allan Cargille, and many others. Many friends at MIT also contributed indirectly to this thesis by helping me preserve the sanity necessary to finish it. In particular, I would like to thank Henrique and Regina Malvar, Eduardo and Sonia Misawa, Fernando and Rosinha Frimm, José Jaime da Cruz, Nilton Rennó, Maria Carmen Lemos, João and José Rosa, Simona Piattone and Sang-Mook Lee. I will remember them all as an integral part of my life at MIT. 


\section{Contents}

$\begin{array}{ll}\text { Abstract } & 3\end{array}$

$\begin{array}{ll}\text { Acknowledgments } & 7\end{array}$

$\begin{array}{lr}\text { List of Figures } & 11\end{array}$

1 Introduction $\quad 13$

1.1 An Introduction to Side-Scan Sonar . . . . . . . . . . . . . . . . . 14

1.1 .1 History . . . . . . . . . . . . . . . . . . 15

1.1.2 Principles of Operation . . . . . . . . . . . . . . . 16

1.1.3 Types and Applications of Side-Scan Sonar . . . . . . . . . . . . 19

1.2 Sonograph Distortions . . . . . . . . . . . . . . . . . . . . . . . . . . . . .

1.2.1 Intensity Distortions . . . . . . . . . . . . . 20

1.2.2 Geometric Distortions . . . . . . . . . . . . . . . 21

1.3 Digital Processing of Sonographs . . . . . . . . . . . . . . . . . . . . . . . . . . . . .

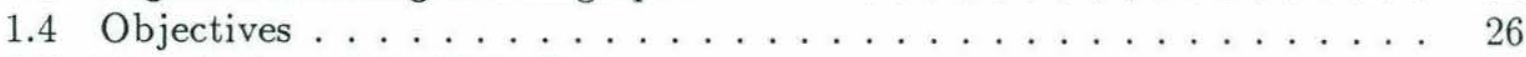

1.5 Description of the Data Set . . . . . . . . . . . . . 27

2 Mathematical Analysis of the Problem $\quad 29$

2.1 Geometric Distortions in Sonographs . . . . . . . . . . . . . . . 29

2.2 Mathematical Model for the Geometric Distortions . . . . . . . . . . . 33

2.2.1 Location of Sampling Points . . . . . . . . . . . . . . . . . 34

2.2.2 Sampling Displacements . . . . . . . . . . . . . . . 37

2.2.3 Linear Model for the Sampling Displacements . . . . . . . . . . . 39

3 Measures of Geometric Distortion in Sonographs 45

3.1 Assumptions on the Statistical Properties of the Sonograph Image . . . . 46

3.2 Measuring Distortion in the Cross-Track Direction . . . . . . . . . . . . 48

3.3 Measuring Distortion in the Along-Track Direction . . . . . . . . . . . 52

3.3.1 Relation Between Correlation Lengths and Sampling Displacements 54

3.3.2 Correlation Lengths in the Absence of Geometric Distortion . . . 59

3.3.3 Detection of Backscanning ............... 64

4 Estimation of Distortion Parameters 69

4.1 Selection of Observation Points . . . . . . . . . . . . . . . . . 69

4.2 Deterministic Least-Squares Estimation . . . . . . . . . . . . . 72 
4.2.1 Estimating the Distortion Parameters . . . . . . . . . . . . 72

4.2.2 Estimating the Attitude Parameters . . . . . . . . . . . . . 75

4.3 Bayesian Linear Least-Squares Estimation . . . . . . . . . . . . . 81

4.3.1 Line-by-Line Estimation . . . . . . . . . . . . . . . . . . 81

4.3 .2 Recursive Estimation . . . . . . . . . . . . . . . . . . \$2

5 Correction of Geometric Distortions $\quad 97$

5.1 Correction of Cross-Track Distortions . . . . . . . . . . . . . . . 97

5.2 Estimating the Sampling Point Coordinates . . . . . . . . . . . . . . 99

5.3 Correcting Geometric Distortions . . . . . . . . . . . . . . . 100

5.3.1 Interpolation in the Cross-Track Direction . . . . . . . . . . . 102

5.3.2 Interpolation in the Along-Track Direction . . . . . . . . . . . 103

5.4 Simulation . . . . . . . . . . . . . . . . . . . . . . 108

5.5 Further Examples . . . . . . . . . . . . . . . . . . . . . . . . 114

6 Final Considerations 125

6.1 Evaluation of Results . . . . . . . . . . . . . . . . . . . . 125

6.2 Suggestions for Future Research . . . . . . . . . . . . . . . 126

6.3 Conclusions . . . . . . . . . . . . . . . . . . . 127

$\begin{array}{lr}\text { A Summary of Notation } & 129\end{array}$

B Derivation of Results from the Geometry of the Problem 133

$\begin{array}{ll}\text { Bibliography } & 137\end{array}$ 


\section{List of Figures}

1.1 Typical operational setting of a side-scan sonar. . . . . . . . . . . . 17

1.2 Example of a side-scan sonar image. . . . . . . . . . . . . . . 18

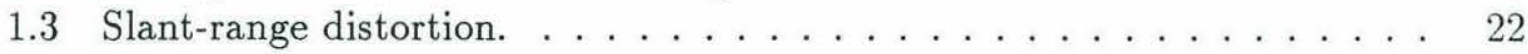

1.4 Rotational instabilities of the towfish. . . . . . . . . . . . . 24

1.5 Location of the survey conducted in the summer of $1987 \ldots \ldots . \ldots 28$

2.1 Coordinate systems defined on the seabed and on the sonograph. . . . . 30

2.2 Sampling patterns on the bottom. . . . . . . . . . . . 32

2.3 Position and orientation of the towfish. . . . . . . . . . . 34

2.4 Relation between a sampling point on the seabed and the location and orientation of the effective beam. . . . . . . . . . . 35

2.5 Sampling displacements in the $(x, y)$ coordinate system. . . . . . . . 38

2.6 Sampling displacements in the $\left(x^{\prime}, y^{\prime}\right)$ coordinate system. . . . . . . . 42

2.7 Technique for estimating sampling point coordinates. . . . . . . . . 44

3.1 Calculation of the sample cross-correlation sequence. . . . . . . . . . 48

3.2 Effect of lateral towfish displacements on the sampling pattern. . . . . . 49

3.3 Estimation of sampling displacements in the cross-track direction. . . . . 50

3.4 Sonograph of Fig. 1.2 after correction of the slant-range distortion. . . . . 51

3.5 Estimated sampling intervals in the cross-track direction. . . . . . . . . 51

3.6 Effect of variations in speed and pitch angle and yaw angle on the sampling pattern. . . . . . . . . . . . . . . 52

3.7 Calculation of positive- and negative-lag correlation lengths. . . . . . . 53

3.8 Examples of the effect of geometric distortions on the correlation length. $\quad 55$

3.9 Relation between the sampling interval and sampling displacements. . . . 56

3.10 Effect of the beam pattern on the correlation length. . . . . . . . . 61

3.11 Estimate of the average correlation length and effect of the sonar beam pattern. . . . . . . . . . . . . . . . 63

3.12 Overall technique for estimating sampling displacements. . . . . . . . 65

3.13 Estimated sampling intervals in the along-track direction. . . . . . . . . 66

3.14 Example of a line affected by backscanning. . . . . . . . . . 67

3.15 Detected backscanning. . . . . . . . . . . . . . . . . . 68

4.1 Example of a line affected by undersampling. . . . . . . . . . . . . 71

4.2 Technique for estimation of the distortion and attitude parameters. . . . 73 
4.3 Estimates of distortion parameters obtained through deterministic leastsquares approximation. . . . . . . . . . . . 76

4.4 Estimates of attitude parameters obtained through deterministic leastsquares approximation. . . . . . . . . . . . . . 80

4.5 The extended least-squares algorithm. . . . . . . . . . . . . . . 88

4.6 Estimates of distortion parameters for the extended least-squares algorithm. 91

4.7 Estimates of attitude parameters for the the extended least-squares algorithm. . . . . . . . . . . . . . . . . . . 92

4.8 Estimates of state-space model parameters for the extended least-squares algorithm. . . . . . . . . . . . . . . . . . . 93

4.9 Estimates of attitude parameters obtained through adaptive Kalman filtering with $N=4$ and $\beta=0.987 \ldots \ldots \ldots \ldots \ldots \ldots$

4.10 Estimates of state-space model parameters for the extended least-squares

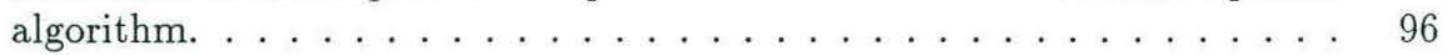

5.1 Height of the water column measured from the sonograph. . . . . . . . 98

5.2 Mesh plot illustrating the geometric distortion in the sonograph. . . . . . 101

5.3 Determining the points for interpolation in the cross-track direction. . . . 102

5.4 Determining the points for interpolation in the along-track direction. . . 104

5.5 Sonograph of Fig. 1.2 after correction of geometric distortions. . . . . . 106

5.6 Comparison of a detail of the original and corrected sonographs. . . . . . 107

5.7 Bilateral estimation of distortion parameters. . . . . . . . . . . . . 109

5.8 Result of independently processing the starboard and port sides of the sonograph. . . . . . . . . . . . . . . . . . . . . 110

5.9 Image used in the simulation. . . . . . . . . . . . . . . . . 111

5.10 Sonograph with simulated geometric distortions. . . . . . . . . . . 112

5.11 Simulated and estimated attitude parameters. . . . . . . . . . . . 113

5.12 Comparison of original, distorted and reconstructed images . . . . . . . 115

5.13 A second sonograph from our data set. . . . . . . . . . . . 116

5.14 Sonograph of Fig. 5.13 after backscanning detection. . . . . . . . . 117

5.15 Sonograph of Fig. 5.13 after correction of geometric distortions. . . . . . 118

5.16 Estimates of attitude parameters for the sonograph of Fig. 5.13. . . . . 119

5.17 A third sonograph from our data set. . . . . . . . . . . . . 121

5.18 Sonograph of Fig. 5.17 after backscanning detection. . . . . . . . . . . . 122

5.19 Sonograph of Fig. 5.17 after correction of geometric distortions. . . . . . 123

5.20 Estimates of attitude parameters for the sonograph of Fig. 5.17. . . . . . 124 


\section{Chapter 1}

\section{Introduction}

This thesis presents a new procedure for the enhancement of acoustic images made by side-scan sonars in the sea and other bodies of water. Specifically, a solution is proposed to the problem of correcting geometric distortions often observed in those images as a result of sonar motion instabilities. The techniques introduced here are unique in that they require no navigational data or attitude measurements for the sonar array to correct the geometric distortions in the image.

The remainder of this chapter presents a more detailed description of the subject of this thesis. An introduction to side-scan sonar is given in Section 1.1, including a brief review of the history of its development, its principles of operation and applications. An example is shown of a side-scan sonar image taken from our data set. Section 1.2 gives a description of the various types of distortions frequently observed in side-scan sonar records, with emphasis on the geometric distortions on which this thesis is focused. The current state of research on digital processing of side-scan sonar data is reviewed in Section 1.3, providing a framework in which the contributions of this thesis can be situated. Section 1.4 states the goals of our work. Finally, Section 1.5 describes the survey during which the data was collected.

Chapter 2 presents the theoretical development of a mathematical model relating geometric distortions in the images to fluctuations in the sonar trajectory and heading. The measurement of the local degree of geometric distortion in small areas of the image from the cross-correlation of line segments is discussed in Chapter 3. Chapter 4 treats 
the estimation of distortion parameters using the model derived in Chapter 2 and the measurements obtained in Chapter 3. In Chapter 5 the parameter estimates are used for correcting the geometric distortions in sonographs and examples of processed images are given. A simulation is used for evaluating the accuracy of the geometric correction. Chapter 6 presents an evaluation of the results obtained in this thesis, along with concluding remarks and suggestions for future research on this problem. Finally, Appendix A presents a summary of the variables defined throughout the thesis, and Appendix B contains the derivation of some of the results from the geometrical analysis carried out in Chapter 2.

\subsection{An Introduction to Side-Scan Sonar}

The development of diving and submersible technology has made the bottom of the sea a stage for a large variety of enterprises. Experiments and studies in marine biology, seabed geology, physical oceanography, and other scientific disciplines are routinely carried out on the seafloor. Archeologists and adventurers alike seek and explore old ship wrecks. Communication and power cables are laid on the bottom while engineering structures are built or deployed on the seabed for extracting oil, gas, and other minerals, or for logistic support of divers. Many of these activities are carried out not only in the sea, but on the bottom of lakes and rivers as well.

The establishment of all these underwater activities has generated an increasing need for underwater remote sensing. This is often provided by acoustic bathymetric devices, such as the basic echo sounder and its more sophisticated multi-beam versions, which have been extensively used to produce bathymetric charts of the oceans. There are, however, many underwater enterprises that require remote sensing at a much finer resolution than can be achieved with bathymetric devices. In some cases, visualization of the bottom can be achieved through underwater photography, filming, or television. However, these means are severely limited by the rapid absorption of light by the water, resulting in maximum ranges of at most a few tens of meters, and sometimes as little as less than a meter, depending on the condition of the water. 
Side-scan sonar bridges the gap between sight and sound in the underwater environment by providing visualization of the bottom through acoustic remote sensing at ranges far beyond those afforded by optical means. It has acquired an increasingly important role in underwater exploration and its wide range of applications includes mapping the seabed as an aid to the production of nautical charts and for oceanographic and underwater archeologic research, search and location of objects on the bottom, fishery studies, support for submersible operations, and off-shore mining and engineering surveys. The history, principles of operation, and applications of side-scan sonars are reviewed in [58], [31], [7], [48], [52], and [3].

\subsubsection{History}

The origins of side-scan sonar can be traced back to the submarine research conducted after World War II in England, where sonar operators of the Allied Submarine Devices Investigation Committee (ASDIC) observed that the intensity of backscattering of highfrequency sonar pulses from the bottom appeared to present a consistent correlation with seabed morphology [24]. Kunze, in 1957 [35], and Chesterman et al., in 1958 [9], were the first to explore this phenomenon in an attempt to produce a representation of the seabed topography. By 1961 the first operational side-scan sonar had been built by the National Institute of Oceanography (NIO) in England, and mounted on the hull of its research ship, RRS Discovery II [59]. The first major seabed survey conducted with that sonar was reported in 1961 [19].

The development of side-scan sonar in the United Kingdom has been carried out by the scientific community, especially by the NIO, now known as the Institute of Oceanographic Sciences (IOS). Their most notable achievement was the Geological Long-Range Inclined Asdic (GLORIA) Project ${ }^{1}$, responsible for the development of a long-range side-scan sonar completed in 1969 [47]. In 1977 the IOS built a second version, the GLORIA II, which incorporated a series of improvements suggested by the experience gained in the operation of the original GLORIA sonar [51].

\footnotetext{
${ }^{1}$ In its early days, side-scan sonar was sometimes called Asdic sonar, because of its association with the Allied Submarine Devices Investigation Committee.
} 
In the United States, the development of side-scan sonar was carried out not only by scientific institutions but also by the industry, and American companies have held a prominent position in the world market from the beginning. Notable examples of sidescan sonars developed by scientific institutions in the United States are the SeaMARC (Sea Mapping and Remote Characterization) sonar and its successor, the SeaMARC II, jointly developed by the University of Hawaii and International Submarine Technology, Ltd.[6]

\subsubsection{Principles of Operation}

Figure 1.1 depicts a typical operational setting for a conventional side-scan sonar. The main components of the system are the so-called towfish, a cylindrical towed body on whose sides are mounted two linear arrays of hydro-acoustic transducers, and the recorder, a piece of equipment containing the circuitry that generates the sonar pulses and detects the returned signal, producing a graphic record of its strength either on paper or on a cathode-ray tube. The arrays of transducers produce two sound beams with very narrow main lobes (typically only 0.2 to 2.0 degrees wide) on each side of the towfish, pointing laterally and down as depicted in the figure. Each sonar pulse ensonifies a narrow strip of the seabed on each side, to a distance of up to 30 kilometers, depending on the operating frequency of the sonar. As the towfish is towed through the water, the lateral sound beams progressively scan a swath of the seabed (thus the name side-scan sonar), while the recorder produces a line-by-line record of the backscattered signal [25].

The visual record produced by a side-scan sonar is called a sonograph. Figure 1.2 shows an example of a sonograph covering an area of $100 \mathrm{~m} \times 200 \mathrm{~m}$ of a fairly flat stretch of seabed consisting of a field of boulders and cables laid on the bottom. The towfish may be pictured moving along the center of the image from bottom to top, so that the right-hand and left-hand halves of the image correspond to the starboard and port sides, respectively. The two arrays of transducers fire a sequence of sound pulses at regular intervals as the towfish is towed above the seabed. After each pulse is emitted, the transducers receive the signals returned from the bottom on the starboard and port sides, before the next pulse is fired. In the case of a digitized sonograph, the returned 


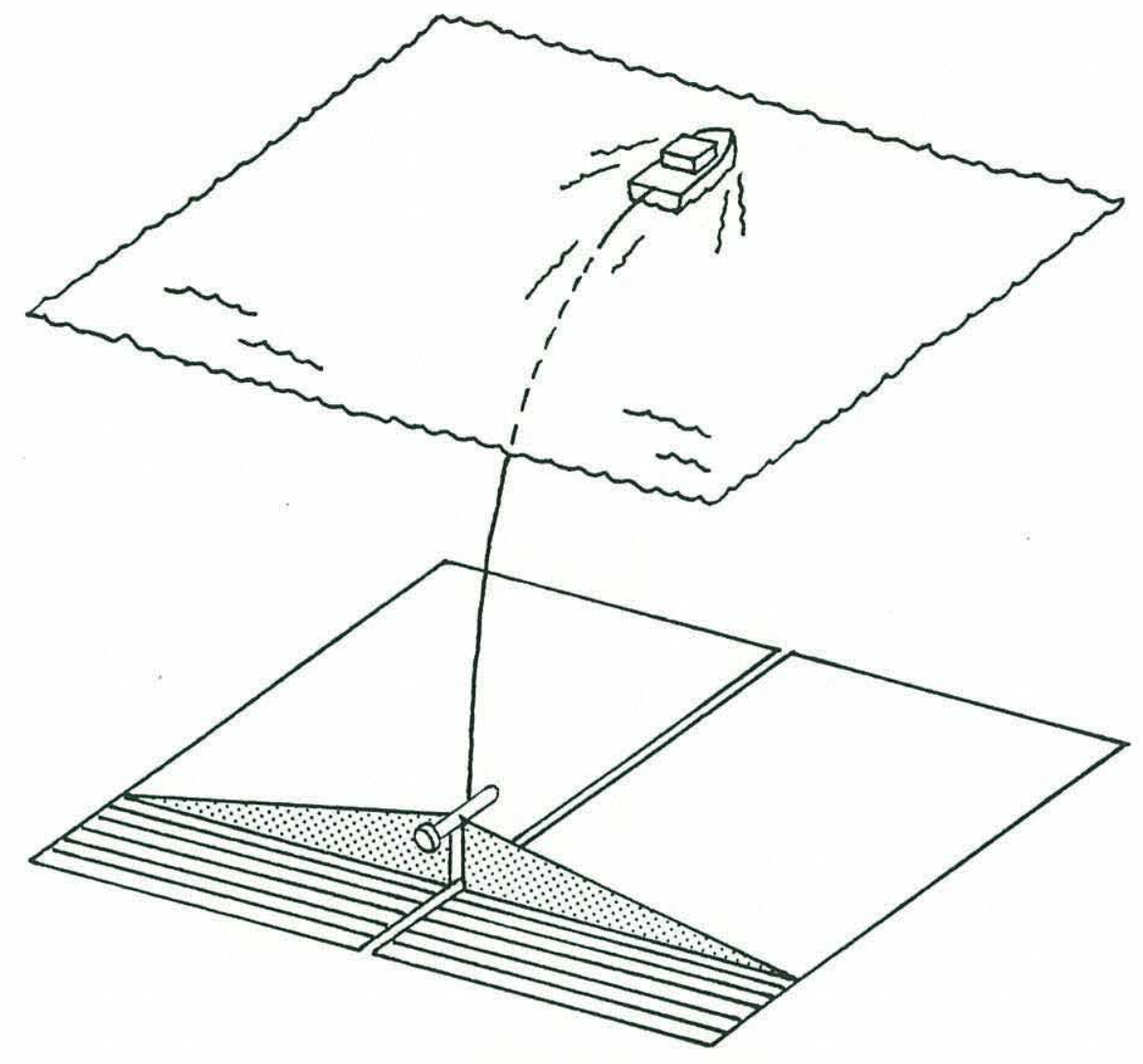

Figure 1.1: Typical operational setting of a side-scan sonar. Lateral sound beams scan the seabed as the sonar towfish is towed above the bottom by a deploying vessel.

signals are sampled at a constant rate to form one line of the image, starting from its middle point and moving towards the sides. The image intensity is proportional to the strength of the returned signal, and the horizontal coordinate measured from the middle of the line corresponds to the time elapsed since the pulse was sent out. Equivalently, the horizontal coordinate may be viewed as range from the towfish, assuming the sound pulse travels at a constant speed. The vertical coordinate of the sonograph corresponds to the distance traveled by the towfish. The dark stripe seen in the middle of the image corresponds to the interval of time between the emission of the sound pulse and the instant it first reaches the seabed, during which there are no reflections except those from fish or other objects in the water column.

As a visual record of backscattered sound, a sonograph depicts a combination of the 

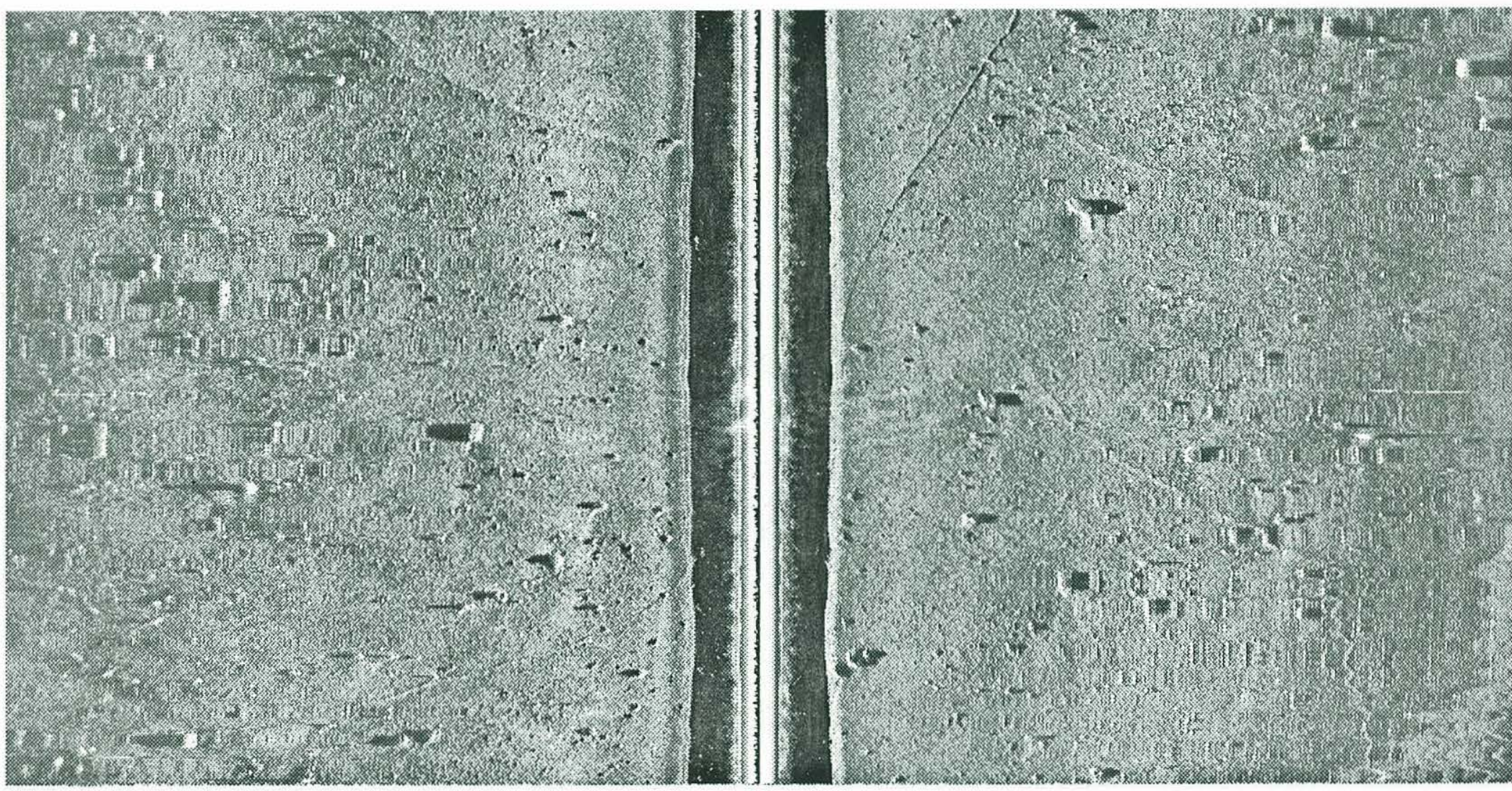

Figure 1.2: Example of a side-scan sonar image, after processing for correction of intensity distortions. Geometric distortions are responsible for the jagged appearance of the cable seen in the lower right-hand corner and for multiple images of several rocks in that area. 
seabed topography and its geological constitution. This is a consequence of the dependence of backscattering strength both on the angle of incidence at which the sound waves reach the target (called the grazing angle) and on the target's ability to reflect them back, that is, its reflectivity [11]. As a result, sonographs resemble aerial photographs, with the difference that the coordinates of a point on a sonograph correspond to horizontal distances in one direction (along-track) and to range to the towfish in the other direction (cross-track), whereas in aerial photographs the coordinates of a point in both directions correspond to horizontal distances on the ground. A closer analogy exists between sonographs and side-looking radar images, which are also formed line-by-line and whose coordinates are also distance and range [50].

\subsubsection{Types and Applications of Side-Scan Sonar}

The most relevant parameter in the characterization of a side-scan sonar is its operating frequency, which fixes its maximum range and, together with the array length and pulse width, determines its resolution. Sound absorption in water increases with frequency, resulting in shorter ranges [11]. However, the beam width and pulse length can be made smaller at higher frequencies, providing increased resolution [60]. Thus, side-scan sonars with low operating frequencies (3.5 to $40 \mathrm{kHz}$ ) have very long ranges (1 to $30 \mathrm{~km}$ on each side) but lower resolution, while high-frequency units (100 to $500 \mathrm{kHz}$ ) have resolutions as fine as a few centimeters at the expense of shorter maximum ranges $(100 \mathrm{~m}$ to $1 \mathrm{~km})$ [24]. The former class of sonars is mostly employed for marine geological and mapping surveys, where it is necessary to cover very large areas of the seabed and only the large-scale topography is of interest. The latter class is better suited to applications such as the visualization of objects on the bottom, which require increased resolution over smaller areas.

A notable example of a long-range side-scan sonar is the GLORIA system, which, with its long total coverage of up to $60 \mathrm{~km}$ (at an operating frequency of $6.5 \mathrm{kHz}$ ) has produced a wealth of data over 15 years of operation by the IOS and on charters to foreign organizations [36]. The United States Geological Survey has employed GLORIA for mapping extensive portions of the US Exclusive Economic Zone (EEZ). An example 
of a high-frequency unit is the $500 \mathrm{kHz}$ Klein Hydroscan sonar, which, with its very fine resolution of less than $5 \mathrm{~cm}$, has produced some striking images of shipwrecks and other objects on the bottom [21].

A number of variations have evolved from the conventional side-scan sonar. Perhaps the most significant of these is the bathymetric side-scan sonar, which uses the phase of the backscattered signal at each moment for calculating the seafloor elevation as a function of range for each line of the sonograph [15] [16] [17]. An example of a bathymetric side-scan sonar is the SeaMARC II [6]. Another variation is the interferometric side-scan sonar [33]. The application of synthetic-aperture techniques to side-scan sonar has also been proposed, but success in this area has been limited [49] [27].

\subsection{Sonograph Distortions}

The distortions that affect sonographs are of two kinds: intensity distortions, which are deviations from the ideal linear relation between image intensity and backscattering strength of the materials on the bottom, ${ }^{2}$ and geometric distortions, which correspond to discrepancies between the relative location of features on the image and their true location on the seabed. Various specific forms of distortions can be identified in each of these two categories [56] [23] [8]. The different types of distortion will be discussed in the remainder of this section.

\subsubsection{Intensity Distortions}

Several types of intensity distortions can be observed in sonographs. One of them is caused by power drop-off in the returned signal, resulting from three factors: The first two are spherical spreading loss and sound absorption by the water, both of which cause the sound wave to be attenuated as it travels away from the towfish and back. The third factor is the decrease in backscattering due to diminishing grazing angles at increasingly longer ranges. Most side-scan sonars attempt to correct power drop-off by applying a

\footnotetext{
${ }^{2}$ These are sometimes called radiometric distortions, a term apparently borrowed from the radar remote sensing nomenclature.
} 
time-varying gain to the returned signal, which the user can adjust to obtain an image intensity as uniform as possible. In the process, one may eliminate some legitimate largescale intensity variations caused by a sloping bottom or by differences in the geological constitution of the seabed.

A source of intensity distortions inherent to the sonar itself is the effect of the side lobes of the beam pattern. There are both vertical sidelobes, i. e., those on the plane perpendicular to the axis of the towfish, and horizontal sidelobes, i. e., those outside that plane. The vertical sidelobes beneath the main lobe may be responsible for variations in intensity in the first portion of the returned signal, while those above the main lobe may cause spurious surface reflections. In the cross-track direction, each line of the sonograph is produced by the convolution of the acoustic beam function, defined by the main lobe and horizontal sidelobes, with the backscattering function of the seabed. As a result, each line is not the perfect sample of the bottom that one would desire. This effect is usually more noticeable at far ranges, where the beam spreading may cause the features to appear smeared in the along-track direction. Pitching and yawing of the towfish also result in variations in image intensity by causing misalignment of the transmitting and receiving beams, with a consequent drop in the power level of the returned signal, which translates into striping of the sonograph in the cross-track direction.

Other types of intensity distortions are ultrasonic interference generated by passing ships, electrical interference from other instruments on the ship, and various kinds of artifacts caused by shoals of fish, dense particle suspension in the water, or other disturbances of the medium.

\subsubsection{Geometric Distortions}

One type of geometric distortion is inherent to side-scan sonar: the so-called slantrange distortion, a consequence of the cross-track coordinate of sonographs being range to the towfish rather than horizontal distance on the bottom. This distortion amounts to a range-dependent coordinate transformation that compresses the scale towards the shorter ranges and causes the water column to appear on the sonograph as depicted in Fig. 1.3. If the bottom is assumed to be flat, correcting the slant-range distortion is a 


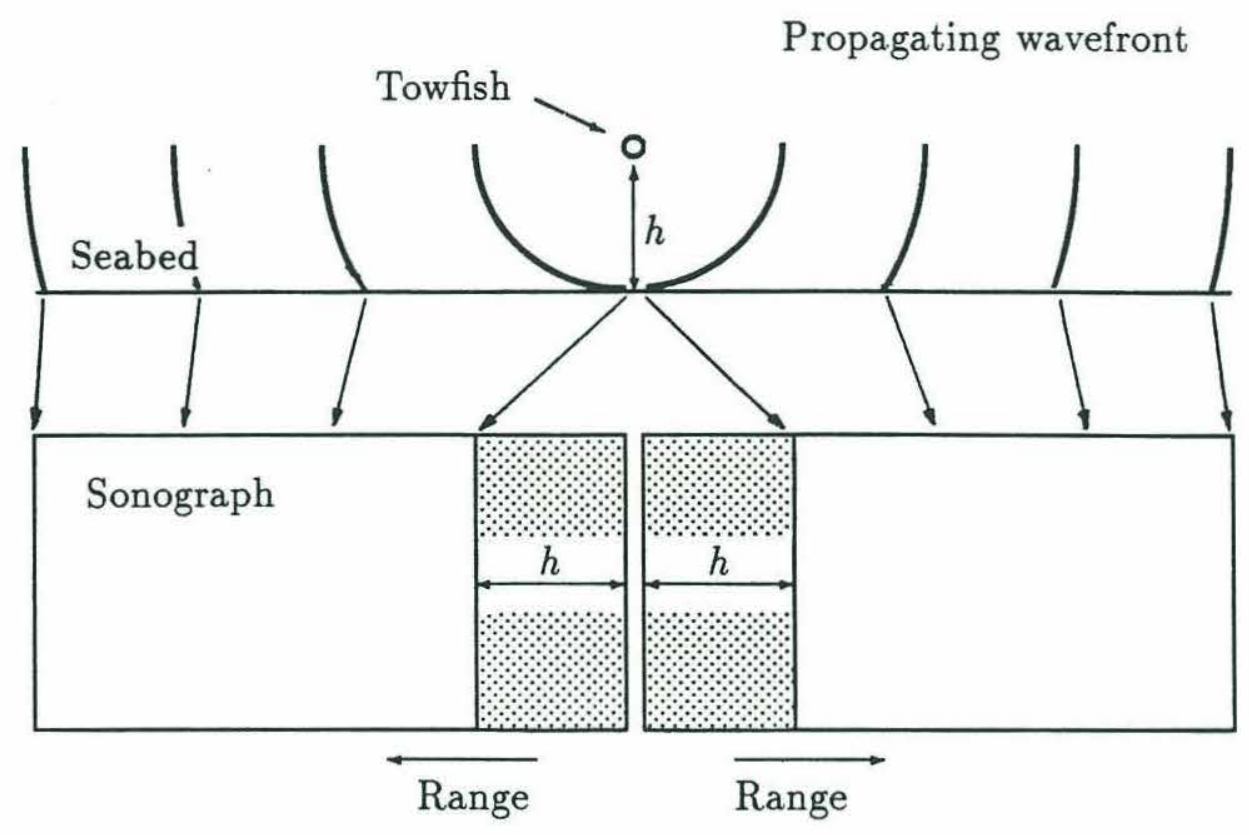

Figure 1.3: The slant-range distortion is caused by the fact that horizontal distances in the sonograph correspond to ranges to the towfish, rather than distances on the seabed.

fairly simple operation that only requires knowledge of the height of the towfish above the bottom. That height can be measured from the sonograph itself as the width of the water column at each line, and then be used to convert ranges to horizontal distances on the bottom. This procedure will be described in greater detail in Section 6.1.

Another source of geometric distortions is variations of the speed of sound in the water, caused by differences in water temperature, pressure, or salinity [11]. Gradients in the velocity of sound in the water also cause the sound waves to be refracted, a phenomenon known as ray bending [18]. Both these phenomena preclude an exact correspondence between sound-wave travel time and actual range to the towfish, with the result that cross-track distances on the sonograph no longer represent actual ranges. Under extreme conditions, ray bending may cause the beam to miss some target areas altogether, leaving shadow zones in the sonograph. These kinds of distortion are usually observed only at longer ranges and are uncommon in high-frequency sonographs.

Still another source of geometric distortion in sonographs is variations in the trajec- 
tory, speed, or orientation of the towfish. Ideally, the towfish would be towed above the bottom at a constant speed, on a straight path, and with the heading always aligned with the trajectory. In practice, however, the towfish is often subject to motion instabilities. Wind and sea currents, for instance, often prevent the deploying vessel from maintaining a constant speed and heading. As a result, the sonograph will display variations in aspect-ratio and other large-scale geometric distortions. These can be corrected by using navigational measurements to determine the true location of the ship and towfish and then resampling the sonograph so as to produce an isometric image. Towfish motion instabilities may also result from the action of underwater currents on the towfish or from the ship sway being communicated to the towfish through the tow cable.

Motion instabilities may be divided into two types: translational and rotational. Translational instabilities correspond to lateral and vertical displacements of the towfish from the desired straight path, and to variations in speed. Rotational instabilities are illustrated in Fig. 1.4. Pitching and yawing produce geometric distortions by causing the beams to scan ahead or back, either simultaneously on both sides, in the case of pitching, or alternating between the port and starboard sides, in the case of yawing. It is common for pitching and yawing to be severe enough to cause the sonar beam to scan backwards over a previously covered area. Objects in a back-scanned area appear in triplicate on the sonograph: one image corresponding to the first time they were scanned, followed by their mirror image reversed in the along-track direction resulting from the back-scanning, and, finally, a third image produced when the beam starts scanning forward again. ${ }^{3}$ Rolling does not produce geometric distortions, but it causes variations in image intensity in the area immediately below the towfish due to the rotation of vertical sidelobes. It can also result in the near-field portion of one side of the sonograph being folded back on itself by allowing the lower part of the corresponding beam to cross over into the opposite side under the towfish.

\footnotetext{
${ }^{3}$ Back-scanning does not occur in the cross-track direction, since it is impossible for the propagating wavefront to reverse its course as a result of towfish instabilities.
} 

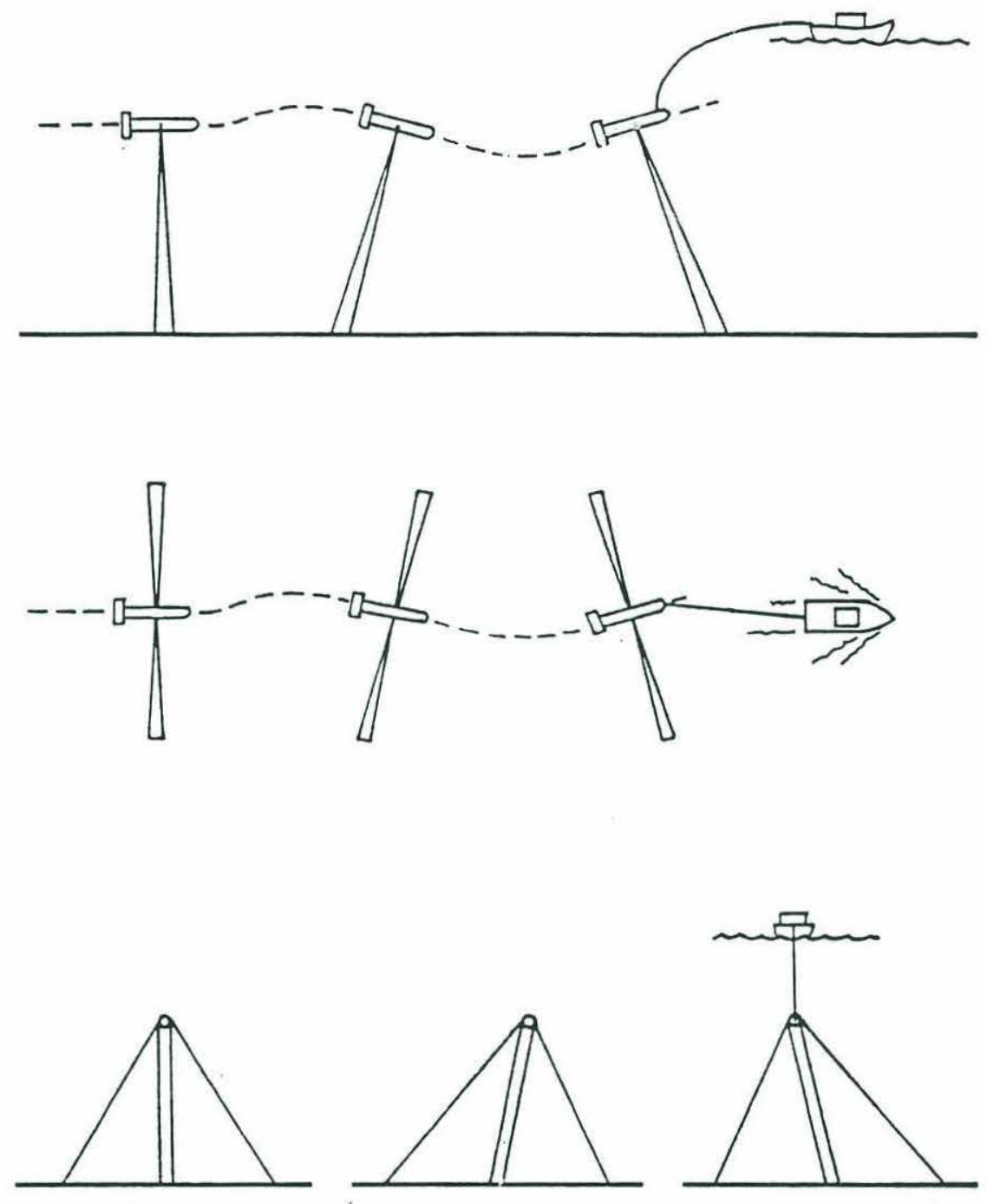

Figure 1.4: Rotational instabilities of the towfish: pitching (top), yawing (middle), and rolling (bottom).

\subsection{Digital Processing of Sonographs}

The various types of distortions described in Section 1.2 can sometimes significantly limit the accuracy of sonographs as visual representations of the seabed. It was not long after the invention of side-scan sonar that the first attempts were made at correcting sonograph distortions. For about a decade, the only types of distortions that were corrected were those caused by variations in ship speed and by the slant-range effect. In [10], 
for instance, a systern was described in which the slant-range distortion was removed by using a nonlinear helix in the recorder, and variations in ship speed were compensated for by using a continuous flow camera to change the along-track scale of the sonograph. The same results were accomplished by fiber optic recorders described in [53] and [61], and by a system reported in [4] that employed a photo-electrical scanner in conjunction with a recorder whose stylus was driven by a stepping motor in a non-linear sweep. Commercial systems that correct for slant range and for variations in ship speed were later developed, such as the ones described in [13] and [32].

Apparently, the first system reported in the literature that departed from the traditional method of recording the sonar signal on paper was the one described in [28] and [29]. In that system the sonar signal was recorded on magnetic tape during the survey and was later displayed on a cathode-ray tube, affording a higher dynamic range than the paper record and at the same time allowing the operator to correct for variations in ship speed and heading by manually adjusting the vertical time base and the slope of the scanning lines.

From the beginning, recorders have incorporated a time-varying gain (TVG), which is applied to the sonar signal in an attempt to compensate for the power drop-off with range caused by sound attenuation, spreading loss, and decreasing grazing angles, as explained in Section 1.2.1. Beyond that, the only other instance of analog processing of sonographs appears to have been the use of analog filtering for selective textural enhancement reported in [34].

Correcting for these simpler geometric and intensity distortions was all that could be accomplished without the recourse of digital processing. The first steps in that direction were taken in 1976, when reports appeared in the literature concerning the digitizing of sonographs [62] and their processing by computers. Paluzzi et al. seem to have been the first to report digital processing of sonographs [41]. Basically, they used existing software developed at the Jet Propulsion Laboratory of the California Institute of Technology for processing images from unmanned planetary exploration missions, and adapted it for sonograph processing. Use of navigational data for correction of large-scale geometric distortions and production of sonograph mosaics was later added to that system, and 
it was employed for processing GLORIA sonographs in a joint project with the British Institute of Oceanographic Sciences [42]. That same software package was later used and extended by Luyendyk, Hajic, and Simonett at the University of California, Santa Barbara [39]. Similar sonograph-processing systems have since been developed at the National Mapping Division of the U. S. Geological Survey as reported by Chavez in [8] and at France's Institut Français de Recherche pour l'Exploitation de la Mer as reported by Augustin in [2]. The systems described in [22], [20], and [46] incorporate techniques for feature extraction and image segmentation and classification. Other instances of digital processing of sonographs are described in [12], [45], [49], [57], [55], and [30].

In general, one might say that considerable improvements are still possible in the field of digital processing for sonograph enhancement. In particular, an effective technique for correcting the geometric distortions caused by towfish instabilities without requiring navigational or attitude measurements undoubtedly constitutes a significant new development.

\subsection{Objectives}

This thesis explores the problem of digitally processing sonographs for the correction of geometric distortions. Its goal is to introduce techniques for estimating and correcting these distortions using solely the information contained in the image itself, without requiring navigational or attitude measurements.

Geometric distortions in the digitized images are interpreted as the result of variations in sampling period and, from the geometry of the problem, a model is derived that relates local variations in the sampling period to parameters that describe deviations in the trajectory and orientation of the towfish. A linearized version of the model is then used for the estimation of these parameters.

Measures of the local degree of geometric distortion of the image are derived from the cross-correlation of segments of adjacent lines. These measurements are not used directly as input to the model, but rather are first processed to yield estimates of the sampling intervals on the seabed, which are in turn used for the estimation of distortion 
parameters. We also examine practical issues concerning the calculation of the sampling period from the correlation length, such as the detection of back-scanning, and the effect of the sonar beam pattern.

Estimation of the distortion parameters from the correlation length is accomplished through deterministic least-squares estimation on a line by line basis. An alternative technique, based on an adaptive Kalman filtering algorithm known as the extended leastsquares method, provides a framework in which a priori knowledge about the towfish dynamics may be easily incorporated in the future. Once the motion parameters are estimated, the geometric distortions are corrected by resampling the image appropriately.

\subsection{Description of the Data Set}

The sonographs used in this thesis, including the one shown in Fig. 1.2, were collected during a survey conducted out of the Woods Hole Oceanographic Institution (IVHOI) in the summer of 1987 . Figure 1.5 shows the location of the survey area off the island of Martha's Vineyard, Massachusetts. That area was chosen because of the existence of utility cables on the bottom, which were expected to facilitate the perception of geometric distortions and thus provide some visual indication of the effectiveness of the technique developed for correcting them. (The technique presented in this thesis, however, does not make any assumptions about or rely in any way on the presence of such objects in the image.)

The survey was conducted aboard WHOI's R.V. Asterias with a high-resolution 500$\mathrm{kHz}$ Klein model $422 \mathrm{~S}-001 \mathrm{E}$ towfish and a Klein model 521 recorder operating at a range of $100 \mathrm{~m}$, the maximum afforded by that sonar unit. The demodulated signal was recorded on magnetic tape and was later digitized through a personal-computer system equipped with a Data Translation DT-2851 "frame-grabber" card. The size of the digitized images is $512 \times 1024$ pixels. Assuming a sound speed of $1500 \mathrm{~m} / \mathrm{s}$, the round-trip time of the sonar pulse corresponding to the maximum range of $100 \mathrm{~m}$ is $133.33 \mathrm{~ms}$, which determined a sampling frequency of $3.9 \mathrm{kHz}$ to produce 512 pixels per line per channel. The resulting sampling distance in the across-track direction is 


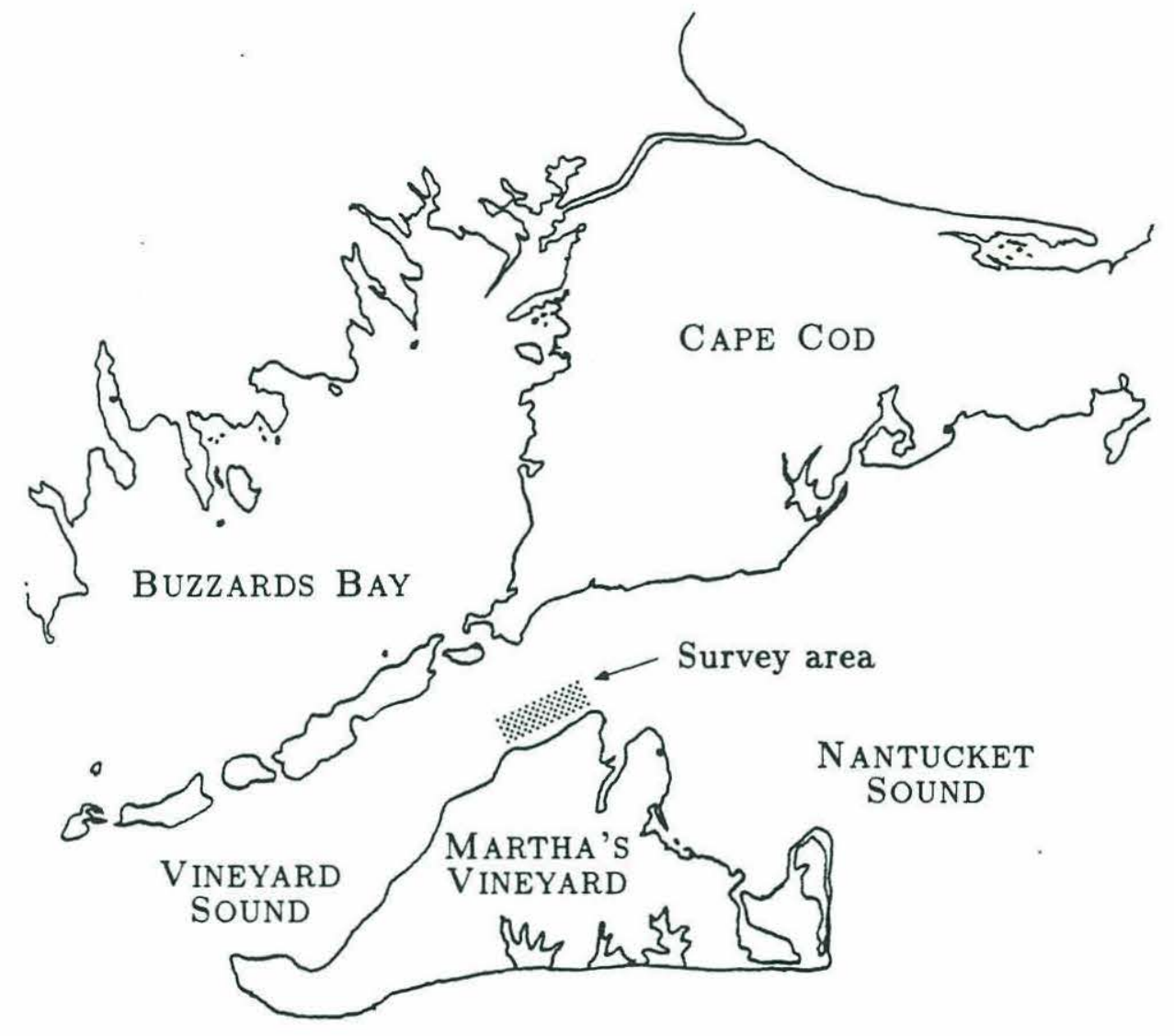

Figure 1.5: Location of the survey conducted in the summer of 1987, during which the sonographs presented in this thesis were collected.

approximately $20 \mathrm{~cm}$. The sonar firing rate was set to 7.5 pulses $/ \mathrm{sec}$, the maximum possible value given the total round-trip travel time of $133.33 \mathrm{~ms}$. The vessel speed was maintained at approximately $3 \mathrm{knots}$, in order to attain the same sampling distance of approximately $20 \mathrm{~cm}$ in the along-track direction as in the cross-track direction, at the selected firing rate.

Though the sea condition during the survey could be described as only moderately rough, the small size of the vessel ( $46 \mathrm{ft}, 20$ long tons loaded displacement) resulted in enough swaying to generate a significant degree of geometric distortion in the images. To that was added the effect of fairly strong undercurrents prevailing in Vineyard Sound. From the resulting set of digitized sonographs a number of images were chosen to be used in the development and evaluation of the techniques described in this thesis. 


\section{Chapter 2}

\section{Mathematical Analysis of the Problem}

This chapter presents a mathematical analysis of geometric distortions in sonographs, leading to the development of a linear model that will be used in Chapter 4 for estimating the location of the sampling points on the seabed. The analysis presented here takes into account the misalignment of the transmitting and receiving beams of the sonar, an effect that is not incorporated in previous studies found in the side-scan sonar literature, such as [37], [14], and [44].

\subsection{Geometric Distortions in Sonographs}

A digitized sonograph can be viewed as a mapping of the backscattering strength of materials on the seafloor onto a monochrome digital image. In order to derive a mathematical description of this transformation it is necessary, first of all, to introduce a three-dimensional system of rectangular coordinates $(x, y, z)$ on the seabed and a two-dimensional rectangular coordinate system $[m, n]$ on the sonograph, ${ }^{1}$ as depicted in Fig. 2.1. For our purposes, the backscattering strength of the bottom can be completely described by a function $b(x, y, z)$ defined on the seabed, and representing the backscattering strength at point $(x, y, z)$. As discussed in Chapter 1 , the backscattering strength

\footnotetext{
${ }^{1}$ The square brackets are used to indicate that $m$ and $n$ are discrete rather than continuous variables.
} 


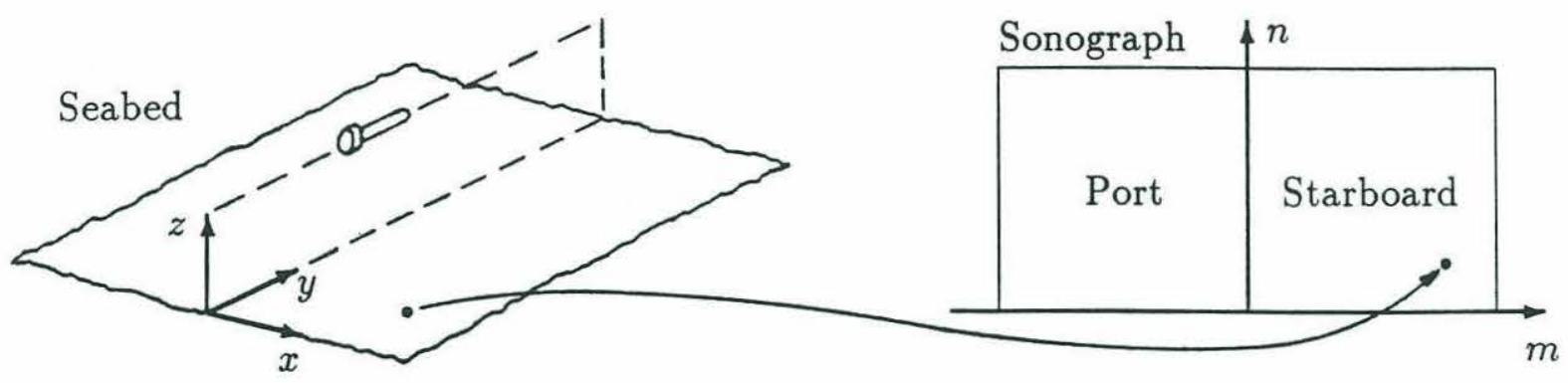

Figure 2.1: Coordinate systems defined on the seabed and on the sonograph.

is also a function of the grazing angle at which the towfish detected the portion of the signal reflected by point $(x, y, z)$ and of the operating frequency of the sonar, although these dependences are not explicitly indicated. The sonograph, on the other hand, can be described by a two-dimensional sequence $s[m, n]$ corresponding to the image intensity at coordinates $[m, n]$. Here $m$ denotes the image coordinate in the cross-track direction, which corresponds to lateral ranges to the towfish, and $n$ denotes the image coordinate in the along-track direction, corresponding to the time elapsed since the beginning of the acquisition of the sonograph. In all sonographs shown in this thesis, the direction of scanning is from bottom to top, so that $n$ is pointing up, while $m$ is positive on the right or starboard side and negative on the left or port side of the image.

The production of a sonograph is then denoted by

$$
b(x, y, z) \mapsto s[m, n] .
$$

This operation actually comprises an intensity transformation $b \mapsto s$ and a sampling $(x, y, z) \mapsto[m, n]$. The intensity transformation is not a simple function of the backscattering coefficient, being affected by a series of factors such as sound attenuation and absorption by the water, the angle of incidence of the wavefront on the bottom, acoustic and electrical noise, and the sonar beam pattern and time-varying gain [52], making it very difficult to describe mathematically. The sampling operation, on the other hand, is considerably easier to describe since the only factors that it involves in addition to the point coordinates are the position and orientation of the towfish. To calculate the true location of points of the sonograph on the seabed, it is necessary to determine an expression not for the direct sampling operation, but for the inverse mapping $[m, n] \mapsto(x, y, z)$. 
Before proceeding, we make a simplifying assumption. Standard side-scan sonars produce no information on the bearing of the returned signal, and it is impossible to determine all three of $x, y$, and $z$ from $m$ and $n$ alone. We therefore make the same assumption generally made for correction of large-scale geometric distortions in sonographs: that the variations in seabed elevation can be disregarded, which corresponds to assuming a flat, horizontal bottom. In that case the variable $z$ can be eliminated, and the geometric mapping is completely described by the two functions,

$$
x=x_{s}[m, n] \text { and } y=y_{s}[m, n],
$$

where $x_{s}[m, n]$ and $y_{s}[m, n]$ are the coordinates of the point on the seabed that was sampled to produce the pixel located at $[m, n]$ in the image. Another simplification is to assume that the image intensity corresponds exactly to the backscattering coefficient, since we are not concerned here with the intensity transformation itself. We can then write

$$
s[m, n]=b(x, y) \mid \begin{aligned}
& x=x_{s}[m, n] \\
& y=y_{s}[m, n]
\end{aligned} .
$$

For the sonograph to provide an undistorted representation of the bottom, $x_{s}[m, n]$ and $y_{s}[m, n]$ would have to be linear functions of $m$ and $n$ with identical linear coefficients. In practiçe, however, they turn out to be nonlinear, due to both the geometry of the physical system and the effect of towfish instabilities. In the next section, explicit formulas will be derived for $x_{s}[m, n]$ and $y_{s}[m, n]$, involving parameters describing the position and orientation of the towfish.

Since $x_{s}[m, n]$ and $y_{s}[m, n]$ are, in general, nonlinear functions, Eq. (2.1) can be interpreted as describing a non-uniform sampling of $b(x, y)$. In fact, plotting the sample locations on the seabed plane does not result in a perfect rectangular grid, but rather in a sampling pattern such as the one shown in Fig. 2.2(a). This figure depicts the case where the towfish follows an ideal straight trajectory at a constant speed and with no pitching or yawing. The dashed line running vertically in the figure represents the projection of the towfish trajectory on the bottom, usually called the bottom track. The other dashed 


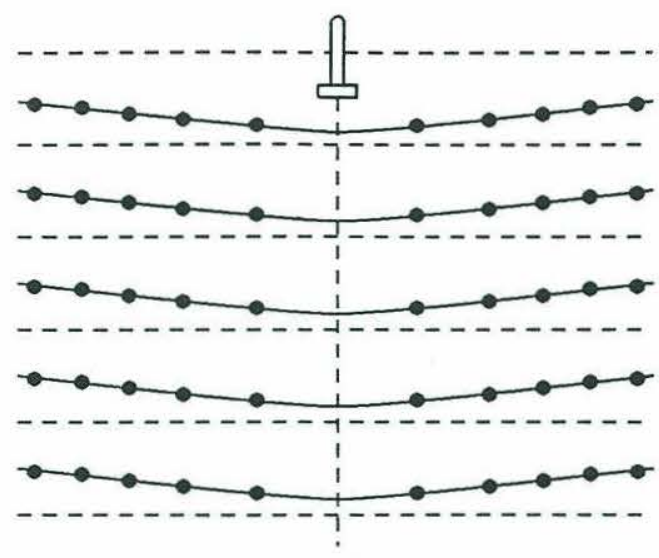

(a)

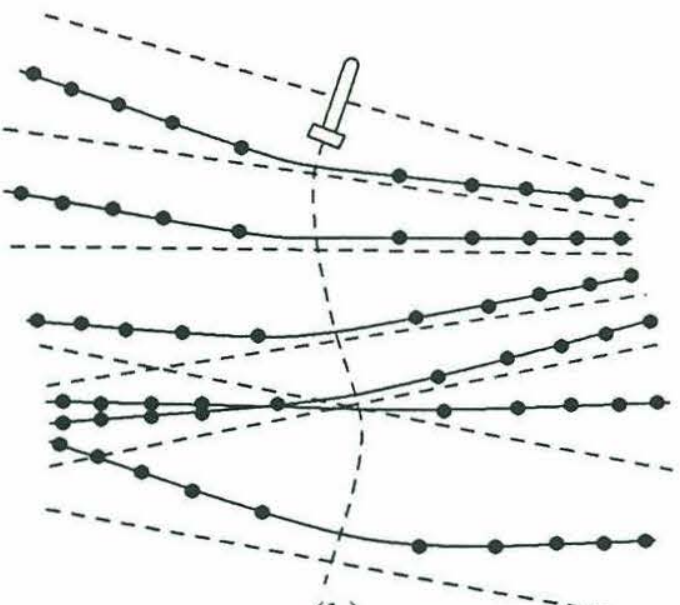

(b)

Figure 2.2: Sampling patterns on the bottom, in the ideal case (a) and in the presence of towfish instabilities (b).

lines running perpendicularly to the bottom track represent the intersection of the axial plane of the transmitting beams with the seafloor. These have fixed positions in space because each sound pulse is emitted at a certain instant in time and then propagates along the direction that the sonar beam had at the instant the pulse was fired. On the other hand, the return from each pulse is received over a period of time during which the receiving beam is not stationary but rather keeps moving along with the towfish at a constant speed. The effective beam pattern is the product of the transmitting and the receiving beam patterns, and if these are the same, which is usually the case for side-scan sonars, then the effective beam can be assumed to lie on the bisecting plane of the axial planes of the transmitting and receiving beams. In other words, each point on the axial plane of the effective beam is assumed to be equidistant to the axial planes of the transmitting beam (which is fixed for each pulse) and of the receiving beam (which keeps moving along with the towfish). As a result, during the time between successive sonar pulses the effective beam scans the bottom at half the speed of the towfish. Each time a pulse is emitted, however, the effective beam jumps ahead to the location of the new transmitting beam, so that its average speed still equals that of the towfish.

As the effective beam moves across the seabed, the signal returned from the bottom 
is sampled at a constant rate as it reaches the transducers. The resulting samples come from points on the bottom that are equally spaced in the along-track $(y)$ direction, since the effective beam is assumed to move at a constant speed between pulses. However, the spacing between sampling points in the cross-track $(x)$ direction varies as a result of the slant-range effect. As a consequence, the sampling points lie on a path that is initially curved, but approaches a straight line at longer ranges, with the decrease of the slant-range effect, as shown in Fig. 2.2(a). Part (b) of the figure shows what the sampling pattern on the bottom might look like when the towfish is subject to translational and rotational instabilities. Because the bottom is not sampled on an ideal rectangular grid but on an irregular pattern, the resulting image will suffer from geometric distortions, in addition to those caused by the slant-range effect. The sampling interval along each scan line and between corresponding points of successive scan lines varies from point to point. Areas where the sampling interval is smaller will look "stretched" with respect to areas where the sampling interval is larger. Furthermore, the image will look skewed whenever scan lines are shifted in the cross-track direction. The figure also indicates how the curves where the sampling points are located may cross, resulting in backscanning.

\subsection{Mathematical Model for the Geometric Distor- tions}

In this section two models will be derived from the geometry of the problem. The first one will express the absolute position of the sampling points on the bottom, $\left(x_{s}[m, n], y_{s}[m, n]\right)$, as a function of the instantaneous values of the towfish attitude parameters, and will be derived with respect to the fixed coordinate system $(x, y, z)$. If measured values of the attitude parameters were available from sensors mounted on the towfish, we would need only substitute these measurements into this first set of equations in order to calculate the coordinates of the sampling points on the bottom and then correct the geometric distortions by resampling the image. In our case, such measurements of the attitude parameters are not available and we will have to seek an alternative way of estimating the sampling point coordinates. To that end, a second model will be 


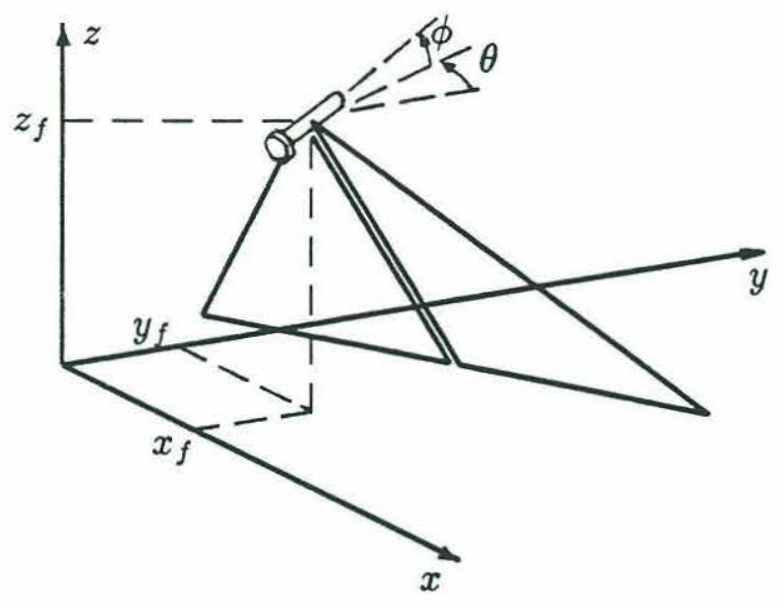

Figure 2.3: The position of the towfish is denoted by $\left(x_{f}, y_{f}, z_{f}\right)$, its pitch angle by $\phi$, and its yaw angle by $\theta$.

derived expressing the relative position of the sampling points as a function of a set of parameters which are closely related to the increments of the attitude parameters between successive firings. This second model will be used in Chapter 4 for estimating the attitude parameters.

\subsubsection{Location of Sampling Points}

The geometry of the problem is depicted in Fig. 2.3. The origin of the coordinate system $(x, y, z)$ is located on the seabed plane, with $y$ pointing in the average direction of the trajectory of the towfish, $x$ pointing to the starboard side, and $z$ in the vertical direction. ${ }^{2}$ The position of the towfish at time $t$ is $\left(x_{f}(t), y_{f}(t), z_{f}(t)\right)$ and its orientation is described by its pitch and yaw angles, denoted $\phi(t)$ and $\theta(t)$, respectively. The pitch angle $\phi(t)$ corresponds to the elevation measured with respect to the horizontal seabed plane and the yaw angle $\theta(t)$ corresponds to the azimuth measured counterclockwise from the $y$ direction. It will not be necessary to consider the towfish roll angle, since, as explained in Chapter 1, rolling does not contribute to geometric distortions. Time $t=0$ corresponds to the instant at which acquisition of the sonograph was initiated, and thus

\footnotetext{
${ }^{2}$ Although in principle this coordinate system may have any arbitrary orientation, it is useful to align the $y$ direction with the trajectory of the towfish so that it may be referred to as the along-track direction and $x$ as the cross-track direction.
} 


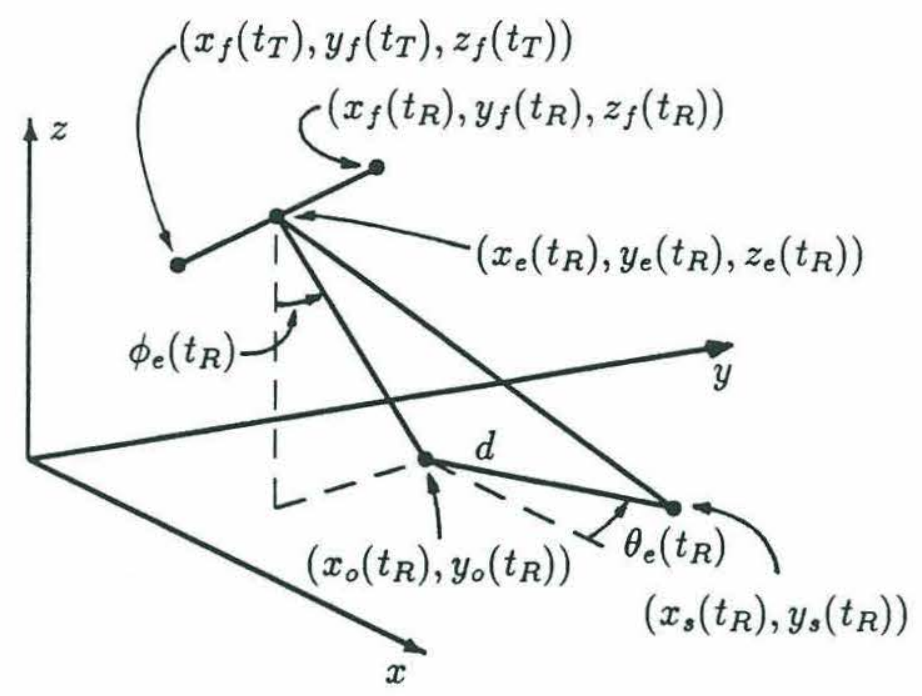

Figure 2.4: Relation between a sampling point on the seabed and the location and orientation of the effective beam.

$\left(x_{f}(0), y_{f}(0), z_{f}(0)\right)$ is the starting point of the towfish trajectory.

Assume a sonar pulse is transmitted at time $t=t_{T}$ and that the returned signal is sampled at time $t=t_{R}$. The effective beam at time $t_{R}$ is the product of the transmitting beam at time $t_{T}$ and the receiving beam at time $t_{R}$, and, as a consequence, its axial plane is located between the axial planes of the transmitting and receiving beams. Therefore, the orientation of the axial plane of the effective beam at time $t_{R}$ is completely defined by the angles

$$
\phi_{e}\left(t_{R}\right) \triangleq \frac{\phi\left(t_{T}\right)+\phi\left(t_{R}\right)}{2} \quad \text { and } \quad \theta_{e}\left(t_{R}\right) \triangleq \frac{\theta\left(t_{T}\right)+\theta\left(t_{R}\right)}{2}
$$

which we call the effective pitch and yaw angles, respectively. Additionally, that plane contains the point $\left(x_{e}\left(t_{R}\right), y_{e}\left(t_{R}\right), z_{e}\left(t_{R}\right)\right)$, where

$x_{e}\left(t_{R}\right) \triangleq \frac{x_{f}\left(t_{T}\right)+x_{f}\left(t_{R}\right)}{2}, \quad y_{e}\left(t_{R}\right) \triangleq \frac{y_{f}\left(t_{T}\right)+y_{f}\left(t_{R}\right)}{2}, \quad$ and $\quad z_{e}\left(t_{R}\right) \triangleq \frac{z_{f}\left(t_{T}\right)+z_{f}\left(t_{R}\right)}{2}$.

In Fig. 2.4 the effective beam is represented by a triangular section of its axial plane. For simplicity, only the starboard beam is shown. The point of the seabed from which the signal sampled at time $t_{R}$ was reflected is the sampling point $\left(x_{s}\left(t_{R}\right), y_{s}\left(t_{R}\right)\right)$. For now $x_{s}$ and $y_{s}$ will be considered as continuous functions of time. This sampling point 
is located on the line defined by the intersection of the axial plane of the effective beam with the seabed plane. The projection of point $\left(x_{e}\left(t_{R}\right), y_{e}\left(t_{R}\right), z_{e}\left(t_{R}\right)\right)$ onto that line is denoted by $\left(x_{o}\left(t_{R}\right), y_{o}\left(t_{R}\right)\right)$, and the distance between this point and the sampling point $\left(x_{s}\left(t_{R}\right), y_{s}\left(t_{R}\right)\right)$ is denoted by $d$. From the geometric arrangement depicted in Fig. 2.4 we see that the equations we seek are

$$
\begin{aligned}
& x_{s}\left(t_{R}\right)=x_{o}\left(t_{R}\right)+d \cos \theta_{e}\left(t_{R}\right) \\
& y_{s}\left(t_{R}\right)=y_{o}\left(t_{R}\right)+d \sin \theta_{e}\left(t_{R}\right) .
\end{aligned}
$$

Now we need only write $x_{o}\left(t_{R}\right), y_{o}\left(t_{R}\right)$ and $d$ as a function of the towfish attitude parameters at times $t_{T}$ and $t_{R}$.

From Fig. 2.4 it is easy to see that

$$
\begin{aligned}
& x_{o}\left(t_{R}\right)=x_{e}\left(t_{R}\right)-z_{e}\left(t_{R}\right) \tan \phi_{e}\left(t_{R}\right) \sin \theta_{e}\left(t_{R}\right) \\
& y_{o}\left(t_{R}\right)=y_{e}\left(t_{R}\right)+z_{e}\left(t_{R}\right) \tan \phi_{e}\left(t_{R}\right) \cos \theta_{e}\left(t_{R}\right) .
\end{aligned}
$$

As for $d$, in Appendix B it is shown that ${ }^{3}$

$$
d=\frac{e f-g r^{2} \pm 2 r \sqrt{16 r^{4}+4\left(g^{2}-e^{2}-4 h^{2}\right) r^{2}+f^{2}+2 e f g+4 e^{2} h^{2}}}{2\left(r^{2}-e^{2}\right)},
$$

where

$$
\begin{aligned}
r \triangleq & \frac{c\left(t_{R}-t_{T}\right)}{2} \\
e \triangleq & {\left[x_{f}\left(t_{R}\right)-x_{f}\left(t_{T}\right)\right] \cos \theta_{e}\left(t_{R}\right)-\left[y_{f}\left(t_{R}\right)+y_{f}\left(t_{T}\right)\right] \sin \theta_{e}\left(t_{R}\right) } \\
f \triangleq & {\left[x_{f}\left(t_{T}\right)-x_{o}\left(t_{R}\right)\right]^{2}+\left[y_{f}\left(t_{T}\right)-y_{o}\left(t_{R}\right)\right]^{2}+z_{f}^{2}\left(t_{T}\right) } \\
& \quad-\left[x_{f}\left(t_{R}\right)-x_{o}\left(t_{R}\right)\right]^{2}-\left[y_{f}\left(t_{R}\right)-y_{o}\left(t_{R}\right)\right]^{2}-z_{f}^{2}\left(t_{R}\right) \\
g \triangleq & {\left[x_{f}\left(t_{R}\right)+x_{f}\left(t_{T}\right)-2 x_{o}\left(t_{R}\right)\right] \cos \theta_{e}\left(t_{R}\right)+\left[y_{f}\left(t_{R}\right)+y_{f}\left(t_{T}\right)-2 y_{o}\left(t_{R}\right)\right] \sin \theta_{e}\left(t_{R}\right) } \\
h^{2} \triangleq & \frac{1}{2}\left(\left[x_{f}\left(t_{T}\right)-x_{o}\left(t_{R}\right)\right]^{2}+\left[y_{f}\left(t_{T}\right)-y_{o}\left(t_{R}\right)\right]^{2}+z_{f}^{2}\left(t_{T}\right)\right. \\
& \left.+\left[x_{f}\left(t_{R}\right)-x_{o}\left(t_{R}\right)\right]^{2}+\left[y_{f}\left(t_{R}\right)-y_{o}\left(t_{R}\right)\right]^{2}+z_{f}^{2}\left(t_{R}\right)\right) .
\end{aligned}
$$

\footnotetext{
${ }^{3}$ In these, as in all subsequent equations, in any compound signs (i. e., \pm or $\mp$ ) the upper sign applies to the starboard side and the lower one to the port side of the sonograph.
} 
The sampling coordinates corresponding to the points of the digitized sonograph can be obtained from Eqs. (2.4a) and (2.4b) by taking $t_{T}=n T_{f}$ and $t_{R}=n T_{f}+m T_{s}$, for $n=0,1, \ldots N_{n}-1, m=0, \pm 1, \ldots, \pm\left(N_{m}-1\right)$, where $T_{f}$ is the sonar firing period, $T_{s}$ is the sampling period used in digitizing the returned signals, $N_{n}$ is the number of lines in the sonograph, and $N_{m}$ is the number of pixels per line on each half of the sonograph. As previously stated, if the towfish attitude parameters are available, as is the case when the towfish is equipped with attitude sensors, the equations derived in this section can be used to calculate the true coordinates of each sampling point on the seabed and the image may then be resampled to rectify the geometric distortions.

\subsubsection{Sampling Displacements}

In the absence of measured values of the towfish attitude parameters, we will have to rely on estimates of the local degree of geometric distortion obtained from the image itself. The slant-range distortion can be easily corrected, as we will see in Section 5.1, and, therefore, we need only be concerned with correcting the distortions due to towfish instabilities. For the remainder of this chapter, we will assume that the slant-range effect has been corrected to produce from the original sonograph a new image $\bar{s}[l, n]$, whose cross-track coordinate is no longer range to the towfish but rather horizontal distances on the bottom. The $l$ coordinate thus corresponds to the value in pixels of the distance $d$ defined in Fig. 2.4 and given by Eq. (2.6), in the same way as the $m$ coordinate of the original image corresponded to range to the towfish in pixels.

The coordinates of the sampling points on the seabed associated with the pixels of $\bar{s}[l, n]$ are denoted by $x_{s}[l, n]$ and $y_{s}[l, n]$. Our measures of geometric distortion in the new image will be estimates of the differences in the values of $x_{s}[l, n]$ and $y_{s}[l, n]$ between sampling points at fixed values of $l$ from one line of the image to the next. These differences are denoted by

$$
\begin{aligned}
& \Delta_{n} x_{s}[l, n] \triangleq x_{s}[l, n+1]-x_{s}[l, n] \\
& \Delta_{n} y_{s}[l, n] \triangleq y_{s}[l, n+1]-y_{s}[l, n]
\end{aligned}
$$

and are indicated in Fig. 2.5. Henceforth $\Delta_{n} x_{s}[l, n]$ and $\Delta_{n} y_{s}[l, n]$ will be referred to 


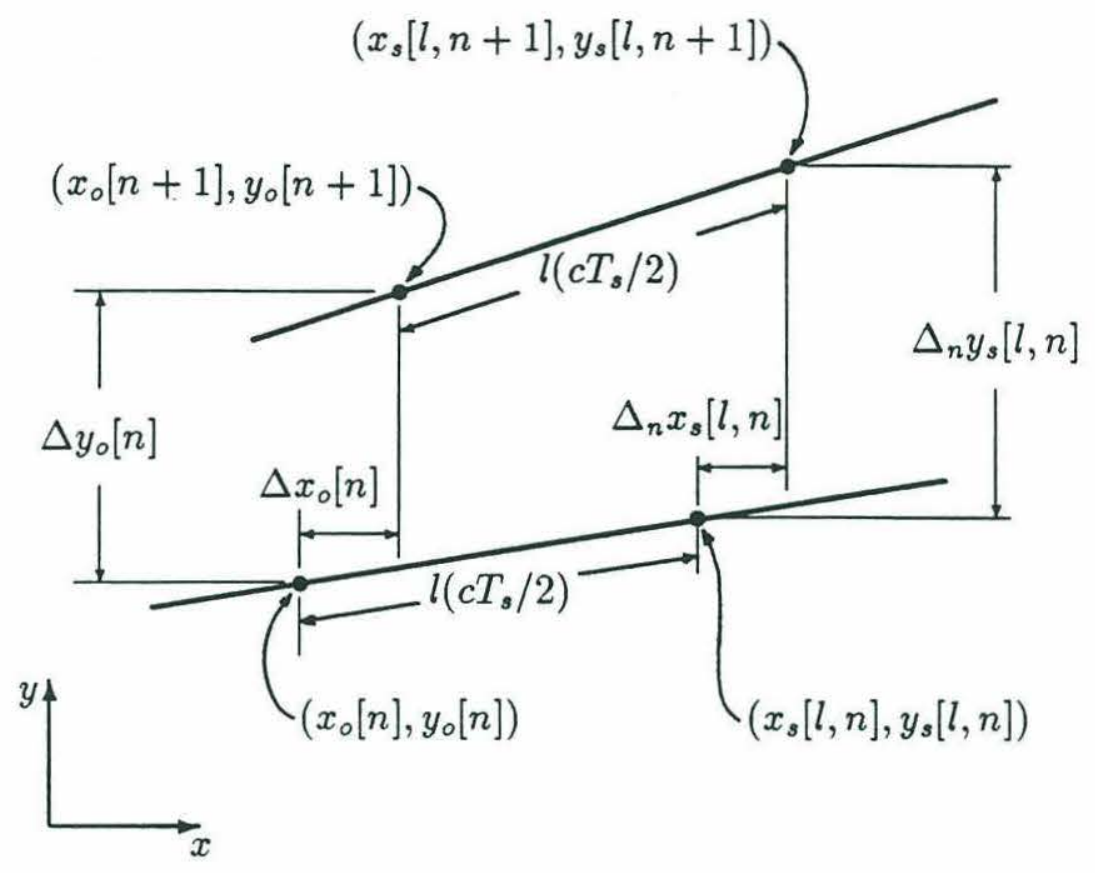

Figure 2.5: The variation in sampling coordinates from one line of the image to the next is described by the sampling displacements $\Delta_{n} x_{s}^{\prime}[l, n]$ and $\Delta_{n} y_{s}^{\prime}[l, n]$, shown here in the $(x, y)$ coordinate system.

as the sampling displacements in the $x$ and $y$ directions, respectively. We will see in Chapter 3 how they can be estimated from the image.

If the estimates of $\Delta_{n} x_{s}[l, n]$ and $\Delta_{n} y_{s}[l, n]$ obtained through the procedure developed in Chapter 3 were accurate enough, then the geometrical analysis of the preceding section would not be necessary for the correction of the geometric distortions in the sonograph. Indeed, starting from an initial guess of the value of the sampling coordinates for the first line of the sonograph, $x_{s}[m, 0]$ and $y_{s}[m, 0], m=0, \pm 1, \ldots, \pm\left(N_{m}-1\right)$, the sampling coordinates of the other lines could be calculated on a line-by-line basis by recursively adding the estimates of sampling displacements $\Delta_{n} x_{s}[l, n]$ and $\Delta_{n} y_{s}[l, n]$. In that way, the sampling coordinates $x_{s}[l, n]$ and $y_{s}[l, n]$ would be calculated for all points of the sonograph, and could then be used for correcting the geometric distortions. However, we will see in Chapter 3 that the estimates of sampling displacements are not accurate enough to be used in this way, and that in order to achieve accurate correction of the 
geometric distortions it will be necessary to apply to these estimates a lower-dimensional model derived from the geometry of the problem. If, from the estimates of sampling displacements, we then estimate the parameters of this lower-dimensional model, the decrease in the dimensionality of the model can be expected to reduce the estimation error of the sampling point coordinates. That amounts to applying to the data our a priori knowledge of the structure imposed on the sampling operation by the side-scan sonar geometry.

In order to apply the model derived in the previous section to the measures of sampling displacements, it would be necessary to combine Eqs. (2.7a) and (2.7b) with Eqs. (2.4a) and $(2.4 \mathrm{~b})$, after making the conversion from $m$ to $l$ prompted by the correction of the slant-range distortion. This operation would result in a set of non-linear equations expressing $\Delta_{n} x_{s}[l, n]$ and $\Delta_{n} y_{s}[l, n]$ as a function of the towfish attitude parameters at times $t_{T}$ and $t_{R}$. At that point, those parameters could be assumed to vary linearly between pulse firings, so that the sampling displacements for each line of the sonograph may be expressed as a function of the increments of the five attitude parameters between successive pulses. With that, the dimensionality of our model would be reduced from $N_{m}$, the number of samples per line of the sonograph, to only five. Deriving these non-

linear equations, however, would be quite involved and using them for estimating the increments of attitude parameters would pose a costly non-linear estimation problem. A more practical approach is to derive an approximate linear model, whose parameters can be more easily estimated through linear recursive estimation techniques. Such a model is derived next.

\subsubsection{Linear Model for the Sampling Displacements}

The first approximation required for deriving a linear model for the sampling displacements is to assume that the towfish remains stationary during the time it receives the returned signals for each pulse. In that case the effective beam also remains fixed during that interval of time and all the sampling points fall on the line defined by the intersection 
of its axial plane with the seabed plane. We then have, for $n T_{f} \leq t_{R}<(n+1) T_{f}$,

$$
\begin{gathered}
\phi\left(t_{T}\right)=\phi\left(t_{R}\right)=\phi_{e}\left(t_{R}\right) \triangleq \phi[n] \\
\theta\left(t_{T}\right)=\theta\left(t_{R}\right)=\theta_{e}\left(t_{R}\right) \triangleq \theta[n] \\
x_{f}\left(t_{T}\right)=x_{f}\left(t_{R}\right)=x_{e}\left(t_{R}\right) \triangleq x_{f}[n] \\
y_{f}\left(t_{T}\right)=y_{f}\left(t_{R}\right)=y_{e}\left(t_{R}\right) \triangleq y_{f}[n] \\
z_{f}\left(t_{T}\right)=z_{f}\left(t_{R}\right)=z_{e}\left(t_{R}\right) \triangleq z_{f}[n] .
\end{gathered}
$$

The point $\left(x_{o}\left(t_{R}\right), y_{o}\left(t_{R}\right)\right)$ also remains stationary for each pulse and is now denoted by $\left(x_{o}[n], y_{o}[n]\right)$. With this, Eqs. (2.5a) and (2.5b) become

$$
\begin{aligned}
& x_{o}[n]=x_{f}[n]-z_{f}[n] \tan \phi[n] \sin \theta[n] \\
& y_{o}[n]=y_{f}[n]+z_{f}[n] \tan \phi[n] \cos \theta[n] .
\end{aligned}
$$

The expression for the distance $d$ in this case is considerably simplified. In fact, by making the values of the attitude parameters at times $t_{R}$ and $t_{T}$ coincide, and taking $t_{R}=t_{T}+m T_{s}$, we see from the equations defining the variables that appear in (2.6) that we now have

$$
r=m\left(c T_{s} / 2\right), \quad e=f=g=0
$$

and

$$
h^{2}=\left(x_{f}[n]-x_{o}[n]\right)^{2}+\left(y_{f}[n]-y_{o}[n]\right)^{2}+z_{f}^{2}[n] .
$$

Thus, $h$ is the distance from point $\left(x_{f}[n], y_{f}[n], z_{f}[n]\right)$ to point $\left(x_{o}[n], y_{o}[n]\right)$, and corresponds to the distance the sonar pulse travels before it first reaches the bottom. This distance can be measured directly in the sonograph as the height of the water column in each line. Henceforth, we will denote it by $h[n]$ in order to explicitly indicate the time dependence. Proceeding with our analysis, we see that Eq. (2.6) now reduces to

$$
d= \pm \sqrt{m^{2}\left(c T_{s} / 2\right)^{2}-h^{2}[n]},
$$

which is exactly the relation prompted by the slant-range distortion. Finally, the expressions for the sampling coordinates become

$$
\begin{aligned}
& x_{s}[m, n]=x_{o}[n] \pm \sqrt{m^{2}\left(c T_{s} / 2\right)^{2}-h^{2}[n]} \cos \theta[n] \\
& y_{s}[m, n]=y_{\circ}[n] \pm \sqrt{m^{2}\left(c T_{s} / 2\right)^{2}-h^{2}[n]} \sin \theta[n] .
\end{aligned}
$$


The equivalent expressions for the sampling points of the slant-range corrected image are

$$
\begin{aligned}
& x_{s}[l, n]=x_{o}[n]+l\left(c T_{s} / 2\right) \cos \theta[n] \\
& y_{s}[l, n]=y_{o}[n]+l\left(c T_{s} / 2\right) \sin \theta[n] .
\end{aligned}
$$

The first-order Taylor-series expansion of these equations yields expressions for the sampling displacements as

$$
\begin{aligned}
& \Delta_{n} x_{s}[l, n] \approx\left(x_{o}[n+1]-x_{o}[n]\right)-l\left(c T_{s} / 2\right) \sin \theta[n](\theta[n+1]-\theta[n]) \\
& \Delta_{n} y_{s}[l, n] \approx\left(y_{o}[n+1]-y_{o}[n]\right)+l\left(c T_{s} / 2\right) \cos \theta[n](\theta[n+1]-\theta[n])
\end{aligned}
$$

or

$$
\begin{aligned}
& \Delta_{n} x_{s}[l, n] \approx \Delta x_{o}[n]-l\left(c T_{s} / 2\right) \sin \theta[n] \Delta \theta[n] \\
& \Delta_{n} y_{s}[l, n] \approx \Delta y_{o}[n]+l\left(c T_{s} / 2\right) \cos \theta[n] \Delta \theta[n],
\end{aligned}
$$

where

$$
\begin{aligned}
& \Delta x_{o}[n] \triangleq x_{o}[n+1]-x_{o}[n] \\
& \Delta y_{o}[n] \triangleq y_{o}[n+1]-y_{o}[n] \\
& \Delta \theta[n] \triangleq \theta[n+1]-\theta[n] .
\end{aligned}
$$

Equations (2.12a) and (2.12b) may be modified to eliminate the dependence on the instantaneous value of the yaw angle. Consider the geometrical arrangement for the $n$th sonar pulse, shown in Fig. 2.6. Let us introduce an auxiliary rectangular coordinate system, $\left(x^{\prime}, y^{\prime}\right)$, shown in the figure, with origin at point $\left(x_{o}[n], y_{0}[n]\right)$, with the $x^{\prime}$ axis located on the line defined by the intersection of the axial plane of the effective beam with the seabed plane, and with the $y^{\prime}$ axis pointing in the direction of the present heading of the towfish. Thus, the axes of this new coordinate system are rotated with respect to the original coordinate system by an angle equal to $\theta[n]$, the current yaw angle of the towfish. 


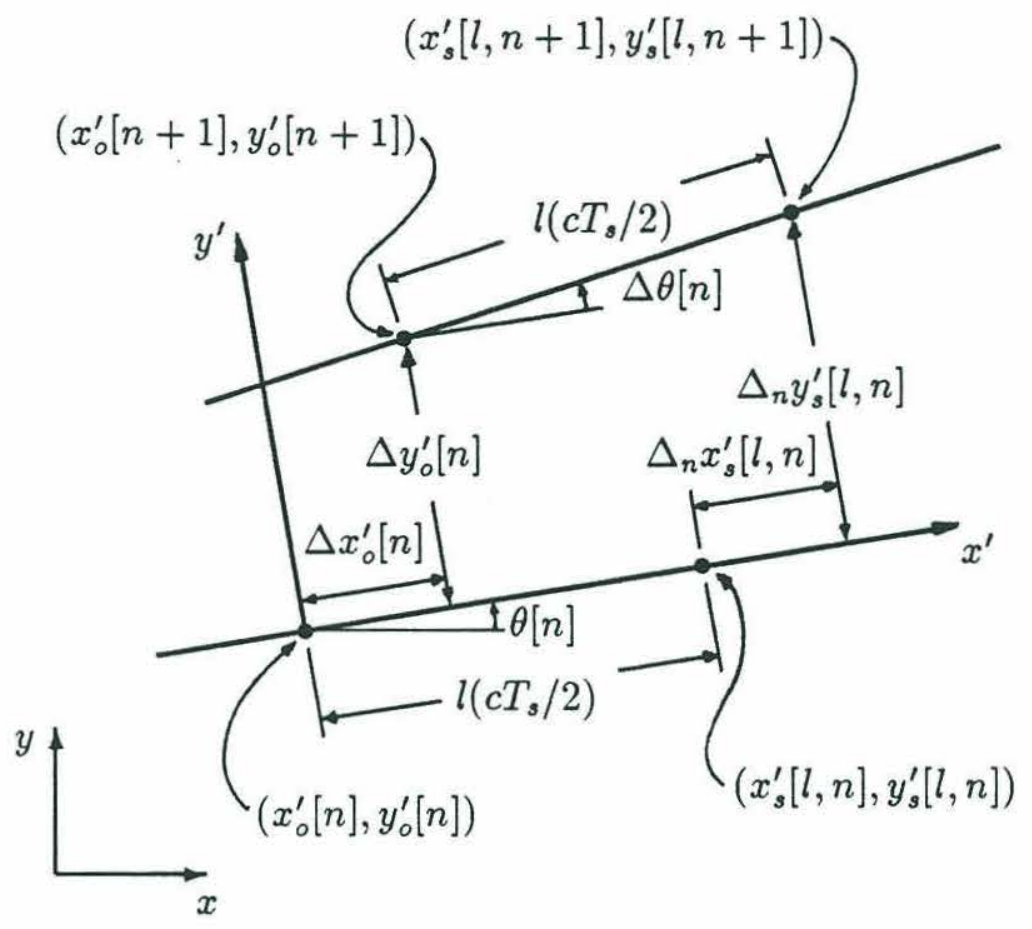

Figure 2.6: Sampling displacements in the $\left(x^{\prime}, y^{\prime}\right)$ coordinate system, defined to have the same orientation as the effective beam for each pulse.

The new and the original coordinate systems are related through

$$
\begin{aligned}
& x^{\prime}=\left(x-x_{\circ}[n]\right) \cos \theta[n]+\left(y-y_{\circ}[n]\right) \sin \theta[n] \\
& y^{\prime}=\left(y-y_{\circ}[n]\right) \cos \theta[n]-\left(x-x_{\circ}[n]\right) \sin \theta[n] .
\end{aligned}
$$

Therefore, the sampling displacements may be converted from one coordinate system to the other through

$$
\begin{aligned}
& \Delta_{n} x_{s}^{\prime}[l, n]=\Delta_{n} x_{s}[l, n] \cos \theta[n]+\Delta_{n} y_{s}[l, n] \sin \theta[n] \\
& \Delta_{n} y_{s}^{\prime}[l, n]=\Delta_{n} y_{s}[l, n] \cos \theta[n]-\Delta_{n} x_{s}[l, n] \sin \theta[n]
\end{aligned}
$$

From these equations and $(2.12 \mathrm{a})$ and $(2.12 \mathrm{~b})$ we obtain the expressions for the sampling displacements in the $\left(x^{\prime}, y^{\prime}\right)$ coordinate system,

$$
\begin{aligned}
& \Delta_{n} x_{s}^{\prime}[l, n]=\Delta x_{o}^{\prime}[n] \\
& \Delta_{n} y_{s}^{\prime}[l, n]=\Delta y_{o}^{\prime}[n]+l\left(c T_{s} / 2\right) \Delta \theta[n],
\end{aligned}
$$


where $\Delta x_{o}^{\prime}[n]$ and $\Delta y_{o}^{\prime}[n]$ correspond to $\Delta x_{o}[n]$ and $\Delta y_{o}[n]$ converted to the new system. This is the lower-order model mentioned before. We refer to $\Delta x_{o}^{\prime}[n], \Delta y_{\circ}^{\prime}[n]$ and $\Delta \theta[n]$ as the distortion parameters to differentiate them from the towfish attitude parameters $x_{f}, y_{f}, z_{f}, \theta$ and $\phi .^{4}$

With the linear model of Eqs. (2.14a) and (2.14b), our task is now reduced to the estimation of three parameters, namely $\Delta x_{o}^{\prime}[n], \Delta y_{o}^{\prime}[n]$, and $\Delta \theta[n]$, for each line of the sonograph from the estimates of sampling displacements. We will see in Chapter 4 how that can be accomplished. The resulting estimates of distortion parameters will then be used for estimating the sampling point coordinates for all points in the image, as explained in Section 5.2. Figure 2.7 illustrates the overall process of estimating the sampling point coordinates. First, the height of the water column is measured in the original sonograph $s[m, n]$ and is then used for correcting the slant-range distortion to produce a new image $\bar{s}[l, n]$. From this new image, estimates of the sampling displacements $\Delta_{n} x_{s}^{\prime}[l, n]$ and $\Delta_{n} y_{s}^{\prime}[l, n]$ are obtained by techniques introduced in Chapter 3 . In order to reduce the estimation error, the linear model of Eqs. (2.14a) and (2.14b) is then applied to the estimates of sampling displacements, yielding estimates of the distortion parameters $\Delta x_{o}^{\prime}[n], \Delta y_{o}^{\prime}[n]$ and $\Delta \theta[n]$. The process is then reversed to yield new estimates of the sampling displacements, which are then converted to the $(x, y)$ coordinate system and added recursively to yield the final estimates of sampling point coordinates. As indicated in the figure, it is also possible to use the estimates of $\Delta x_{o}^{\prime}[n], \Delta y_{o}^{\prime}[n]$ and $\Delta \theta[n]$ for obtaining estimates of the towfish attitude parameters. Though this is not necessary for correcting the geometric distortions, it is convenient to obtain estimates of parameters that describe more directly the location and orientation of the towfish. The estimation of attitude parameters is discussed in Section 4.2.2.

In the next chapter we will consider how to obtain the estimates of sampling displacements from which the distortion parameters and attitude parameters will be estimated in Chapter 4.

\footnotetext{
${ }^{4}$ It might seem more appropriate to call them simply the model parameters, but we will save that designation for the parameters of the state space model introduced in Chapter 4.
} 
Original sonograph

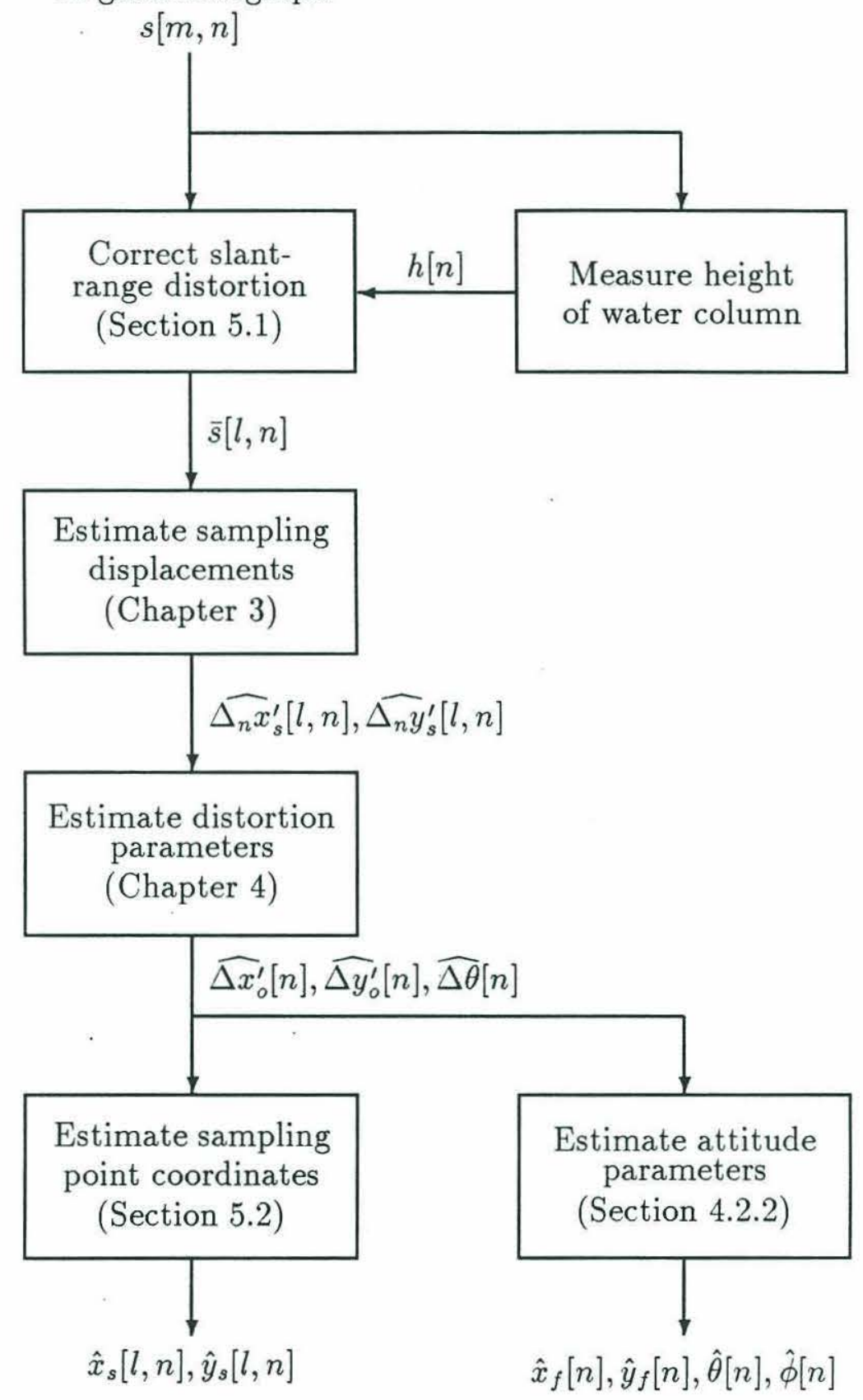

Figure 2.7: Overview of the technique for estimating sampling point coordinates. 


\section{Chapter 3}

\section{Measures of Geometric Distortion}

\section{in Sonographs}

In order to estimate variations in the towfish attitude parameters, it is necessary to extract from a sonograph some measure of local geometric distortion. In this chapter we consider how to obtain such measures and how to estimate from them the sampling displacements $\Delta_{n} x_{s}^{\prime}[l, n]$ and $\Delta_{n} y_{s}^{\prime}[l, n]$ introduced in Chapter 2, from which in turn the distortion parameters $\Delta x_{o}^{\prime}[n], \Delta y_{o}^{\prime}[n]$ and $\Delta \theta[n]$ will be estimated in Chapter 4. Because of the way the seabed is sampled on a raster-scan basis, the predominant type of geometric distortion in the cross-track direction is "skewing," that is, lateral shifting of the lines so that they are not properly aligned. On the other hand, in the along-track direction the predominant type of geometric distortion is "compression" or "stretching" of the image due to variations in the spacing between scan lines on the bottom. Both types of distortions will be measured by cross-correlating segments of adjacent lines of the sonograph. 


\subsection{Assumptions on the Statistical Properties of the Sonograph Image}

Estimating geometric distortions in an image from the image alone requires that certain assumptions be made about the imaged scene. The basic assumption that allows us to obtain measures of geometric distortions in sonographs is that $b(x, y)$, the two-dimensional backscattering function of the bottom introduced in Section 2.1, is a wide-sense stationary random process with isotropic autocorrelation function. Thus, if $R_{b}(\Delta x, \Delta y)$ denotes the autocorrelation function of $b(x, y)$, we have

$$
R_{b}(\Delta x, \Delta y)=R_{b}\left(\sqrt{(\Delta x)^{2}+(\Delta y)^{2}}\right) .
$$

The assumption that the backscattering function is wide-sense stationary may not hold if there are significant variations in the morphology of the bottom. However, the techniques developed here may still be applied to such images if they are divided into areas where the morphology is uniform enough to justify the assumption of wide-sense stationarity. The assumption of isotropism is valid if there is no systematic orientation of the features on the bottom in some direction. Thus, it may not hold in areas containing sand dunes or other formations shaped by undercurrents.

Assuming that the backscattering function is wide-sense stationary and that its autocorrelation function is isotropic, the degree of geometric distortion in the image can be estimated by measuring variations in the shape of the sample autocorrelation sequence of small areas of the image. As explained in the next sections, the sampling displacement $\Delta_{n} x_{s}^{\prime}[l, n]$ is estimated from the degree of skewing in the $l$ direction, of the local sample autocorrelation sequence at point $[l, n]$, and $\Delta_{n} y_{s}^{\prime}[l, n]$ is estimated from the width of the main peak of that sequence in the $n$ direction. The sampling displacements may be expected to be comparable to the inter-pixel distance, and, consequently, computing the autocorrelation sequence only at lags equal to an integer number of pixels would result in too coarse a quantization of their estimates. To achieve greater accuracy in the estimates, the image is upsampled in both directions so that the autocorrelation function may be computed at non-integer lags. The upsampling operation is carried out through 
zero-padding of the discrete Fourier transform of the image. Denoting by $s_{u}\left[l^{\prime}, n^{\prime}\right]$ the upsampled image, by $N_{u}$ the upsampling factor, and by $\mathcal{F}_{N_{1}, N_{2}}$ the $\left(N_{1} \times N_{2}\right)$-point Fourier transform, we have ${ }^{1}$

$$
s_{u}\left[l^{\prime}, n^{\prime}\right]=\mathcal{F}_{N_{u} N_{l}, N_{u} N_{n}}^{-1}\left\{\mathcal{F}_{N_{l}, N_{n}}\{\bar{s}[l, n]\}\right\} .
$$

The local autocorrelation sequence of the upsampled image at point $\left[l^{\prime}, n^{\prime}\right]$ is estimated through the sample autocorrelation sequence of a block of pixels centered at $\left[l^{\prime}, n^{\prime}\right]$. In principle, the averaging could be carried out over blocks extending over two or more lines of the image, but single-line segments are used because geometric distortions can be expected to cause greater variation of the autocorrelation in the along-track direction than in the cross-track direction, as a result of the side-scan sonar geometry. To compensate for local variations in the mean value and standard deviation of the image, the sample correlation coefficient between line segments is used instead of their inner product. Assume that the line segment used in computing the correlation coefficient extends for $L$ pixels on each side of the point being considered (in the original image, before upsampling). Then the local sample mean and standard deviation of the image over that segment are given by ${ }^{2}$

$$
\mu_{s}\left[l^{\prime}, n^{\prime}\right]=\frac{1}{2 L+1} \sum_{k=-L}^{k=L} s_{u}\left[l^{\prime}+k N_{u}, n^{\prime}\right]
$$

and

$$
\sigma_{s}\left[l^{\prime}, n^{\prime}\right]=\left[\frac{1}{2 L+1} \sum_{k=-L}^{k=L}\left(s_{u}\left[l^{\prime}+k N_{u}, n^{\prime}\right]-\mu_{s}\left[l^{\prime}, n^{\prime}\right]\right)^{2}\right]^{\frac{1}{2}} .
$$

The normalized sample cross-correlation between a line segment centered at point $\left[l^{\prime}, n^{\prime}\right]$

\footnotetext{
${ }^{1}$ In practice, only a few rows and columns of the image are upsampled at a time, to reduce the computer memory usage.

${ }^{2}$ Because of the upsampling operation, the sample mean and standard deviation do not change much whether the sums are carried out over a continuous line segment of the upsampled image or only over a smaller number of pixels of that segment separated by the upsampling interval $N_{u}$, as in these equations, resulting in computational savings.
} 


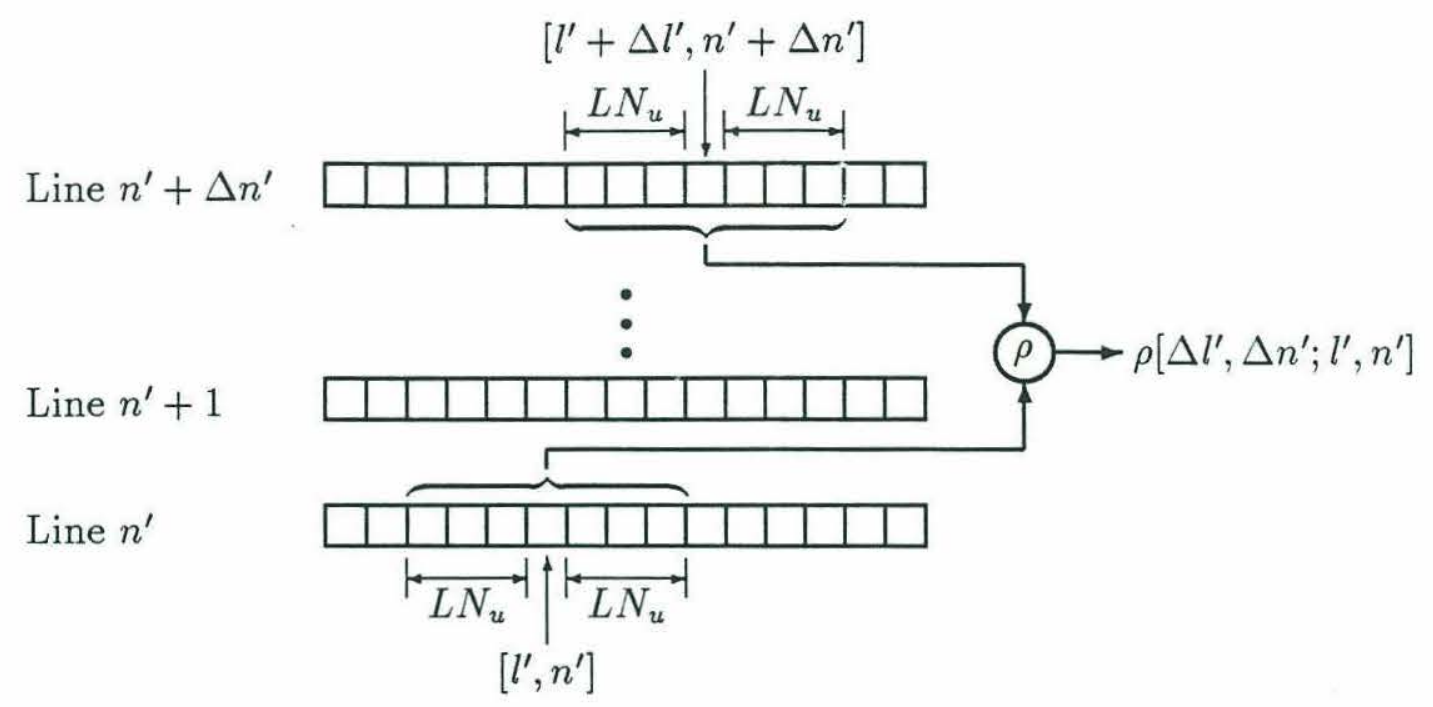

Figure 3.1: Calculation of the sample cross-correlation sequence between line segments, that is used as an estimate of the local autocorrelation sequence of the image. Only every $N_{u}$ th pixel is taken in the segments indicated by braces, so that the averaging is done over $(2 L+1)$ points, for computational savings (see Footnote 2 on page 47 ).

and another segment centered at $\left[l^{\prime}+\Delta l^{\prime}, n^{\prime}+\Delta n^{\prime}\right]$ is given by

$$
\begin{aligned}
\rho\left[\Delta l^{\prime}, \Delta n^{\prime} ; l^{\prime}, n^{\prime}\right]= & \frac{1 /(2 L+1)}{\sigma_{s}\left[l^{\prime}, n^{\prime}\right] \sigma_{s}\left[l^{\prime}+\Delta l^{\prime}, n^{\prime}+\Delta n^{\prime}\right]} \sum_{k=-L}^{k=L}\left[\left(s_{u}\left[l^{\prime}+k N_{u}, n^{\prime}\right]-\mu_{s}\left[l^{\prime}, n^{\prime}\right]\right)\right. \\
& \left.\left(s_{u}\left[l^{\prime}+\Delta l^{\prime}+k N_{u}, n^{\prime}+\Delta n^{\prime}\right]-\mu_{s}\left[l^{\prime}+\Delta l^{\prime}, n^{\prime}+\Delta n^{\prime}\right]\right)\right] .
\end{aligned}
$$

This normalized sample cross-correlation sequence is used as an estimate of the autocorrelation sequence of the image at point $\left[l^{\prime}, n^{\prime}\right]$ and at lags $\Delta l^{\prime}$ and $\Delta n^{\prime}$. A graphical representation of its calculation is given in Fig. 3.1.

\subsection{Measuring Distortion in the Cross-Track Direc- tion}

According to the linear model model derived in Section 2.2.3, the sampling locations corresponding to points of the slant-range corrected image $\bar{s}[l, n]$ are equally spaced along straight lines on the seabed plane. As indicated in that section, this is an approximation 


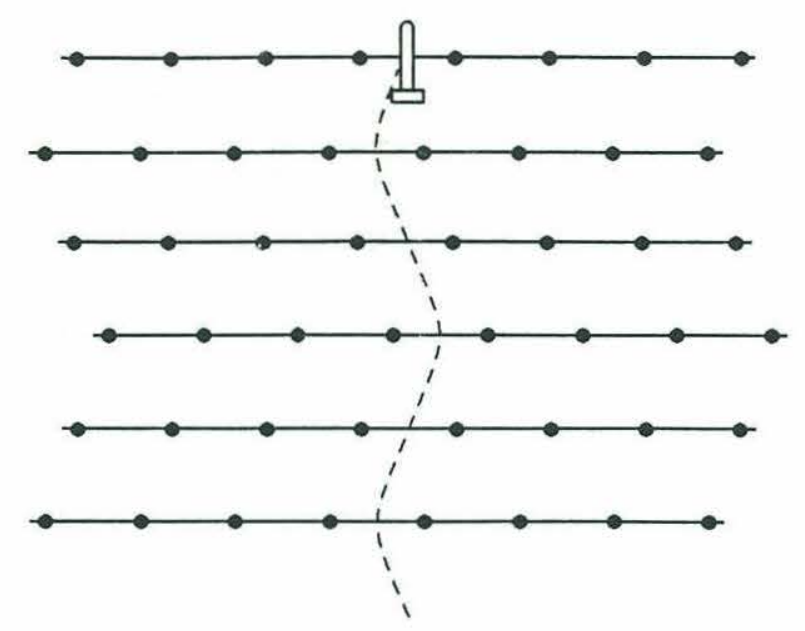

Figure 3.2: Effect of lateral towfish displacements on the sampling pattern. According to the linear model derived in Section 2.2.3, every sampling point on each scan line is shifted laterally by a distance equal to the displacement of the towfish at that line.

that assumes that the towfish remains stationary while receiving the returned signals of each pulse and that the increment in yaw angle between successive pulses is small. The slant-range correction also assumes that the seabed is planar and horizontal. Within the limits of these approximations, distortions in the $l$ (cross-track) direction are caused mainly by lateral displacement of the towfish, and translate into a shift of the sampling points from one line to the next that was described in Chapter 2 by the sampling displacement $\Delta_{n} x_{s}^{\prime}[l, n]$. The resulting geometric distortion in that direction is in the form of skewing rather than compression or stretching of the image, as illustrated in Fig. 3.2. The sampling pattern shown in that figure assumes that the towfish bearing remained fixed in the $y$ direction as it swayed laterally from side to side.

In the absence of geometric distortions, the autocorrelation of the image equals the autocorrelation of the backscattering function of the seabed, which is assumed to be isotropic. In that case its contour lines are concentric circles. However, when the towfish is subject to lateral displacements, the autocorrelation function becomes skewed in the cross-track direction, and its contour lines are turned into ellipses, as shown in Fig. 3.3(a). The sampling displacement $\Delta_{n} x_{s}^{\prime}[l, n]$ determines by how much line $(n+1)$ of the image is shifted with respect to line $n$, and, therefore, is reflected on the displacement of the 

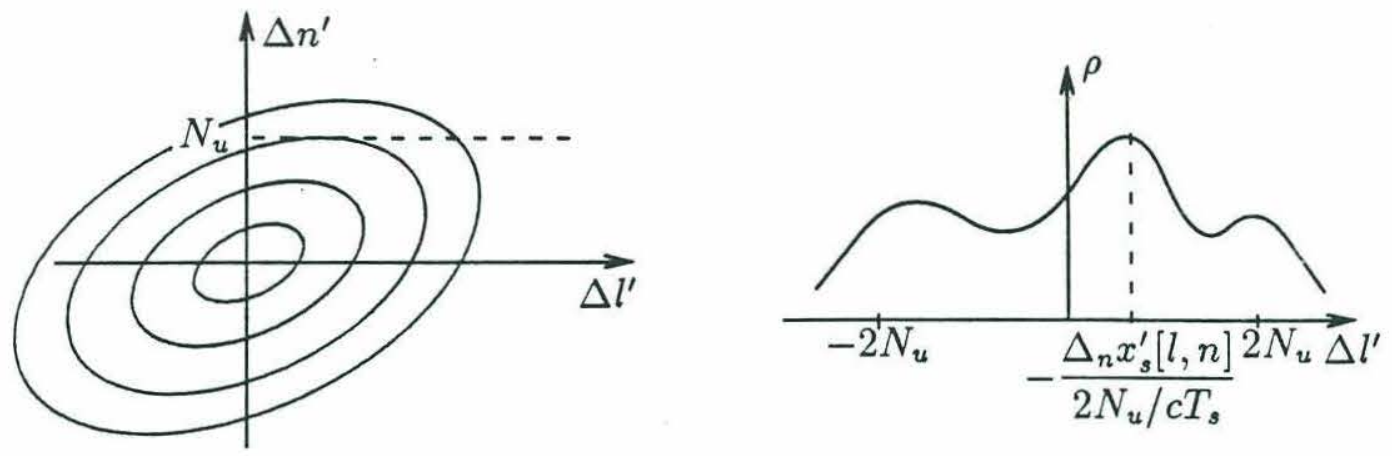

Figure 3.3: Sampling displacements in the cross-track direction, $\Delta_{n} x_{s}^{\prime}[l, n]$, are estimated from the location of the main peak of the cross-correlation sequence $\rho\left[\Delta l^{\prime}, \Delta n^{\prime} ; l N_{u}, n N_{u}\right]$, at an along-track lag $\Delta n^{\prime}=N_{u}$.

maximum point of the local autocorrelation function at a lag $\Delta n=1$ (or $\Delta n^{\prime}=N_{u}$, in the upsampled image). Part (b) of the figure represents a cross-section of $\rho\left[\Delta l^{\prime}, \Delta n^{\prime} ; l^{\prime}, n^{\prime}\right]$ at point $\left[l^{\prime}, n^{\prime}\right]=\left[l N_{u}, n N_{u}\right]$ and at an along-track lag $\Delta n^{\prime}=N_{u}$. The maximum point of this curve provides an estimate of the sampling displacement $\Delta_{n} x_{s}^{\prime}[l, n]$. The search for the maximum is limited to lags between -2 and 2 pixels $\left(-2 N_{u}\right.$ and $2 N_{u}$ pixels in the interpolated image), since it is unlikely that the lateral motion of the towfish between successive pulses would be greater than the corresponding distance of $\pm 40 \mathrm{~cm}$. Thus

$$
\left.\widehat{\Delta_{n} x_{s}^{\prime}}[l, n]=-\frac{c T_{s}}{2 N_{u}} \arg \max \rho\left[\Delta l^{\prime}, N_{u} ; l N_{u}, n N_{u}\right] . \Delta l^{\prime} \leq 2 N_{u}\right] .
$$

The $\left(c T_{s} / 2 N_{u}\right)$ factor is required for the conversion from $l^{\prime}$ (upsampled image coordinates in pixels) to $x^{\prime}$ (distances on the seafloor in meters), and the negative sign is included because, if line $(n+1)$ seems to be shifted in one direction with respect to line $n$, then the sampling points must have shifted in the opposite direction on the bottom from ane scan to the next.

If the sonograph of Fig. 1.2 is processed as explained in Section 5.1 to eliminate the slant-range distortion, we obtain the image shown in Fig. 3.4, from which the estimates of $\widehat{\Delta_{n} x_{s}^{\prime}}[l, n]$ are calculated as described above. The resulting estimates are shown in Fig. 3.5. The cross-correlations were calculated with $L=3$, i. e., with a window size of seven pixels, and at lag increments of $1 / 128$ pixel. The image seems to contain primarily the noise 


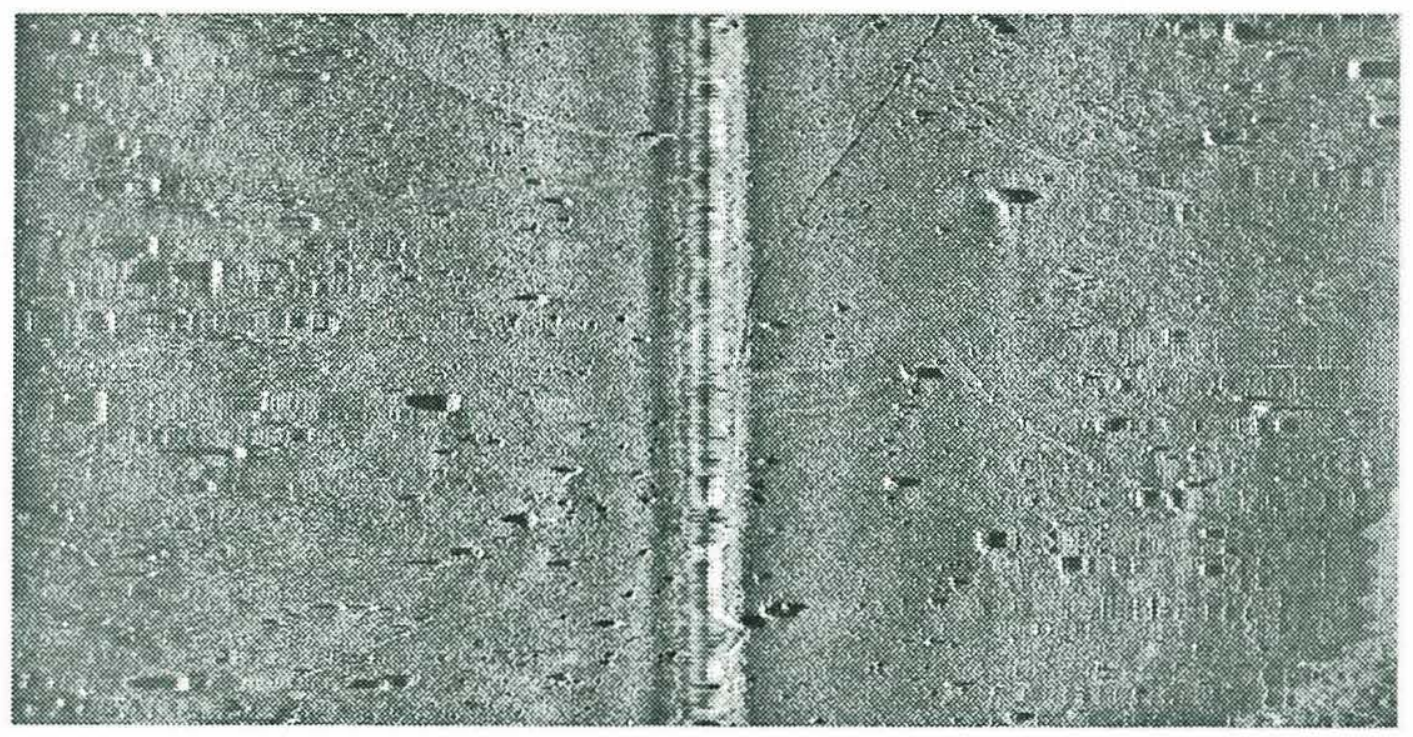

Figure 3.4: Sonograph of Fig. 1.2 after correction of the slant-range distortion, as explained in Section 5.1.
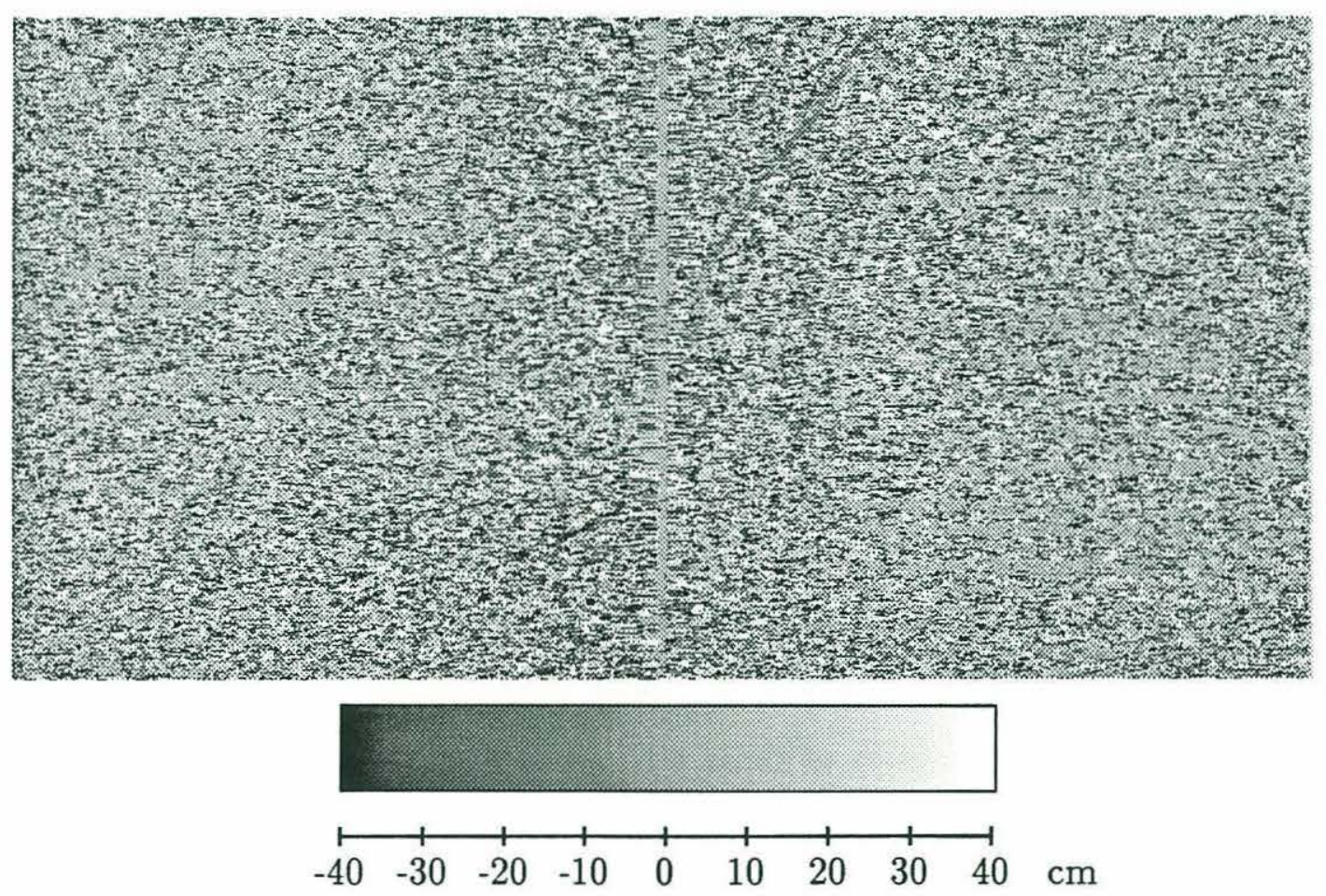

Figure 3.5: Estimates of $\Delta_{n} x_{s}^{\prime}[l, n]$ for the sonograph of Fig. 3.4. The image intensity at each point is proportional to the estimate $\widehat{\Delta_{n} x_{s}^{\prime}}[l, n]$ at that point, according to the tone scale shown above. 


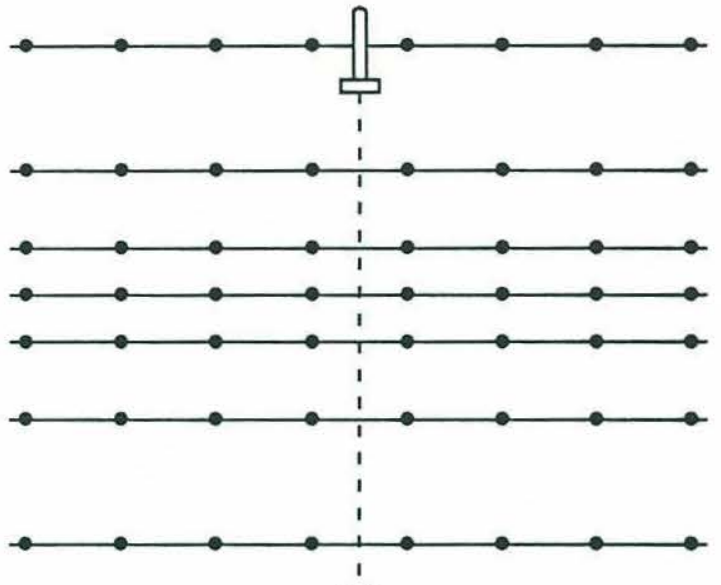

(a)

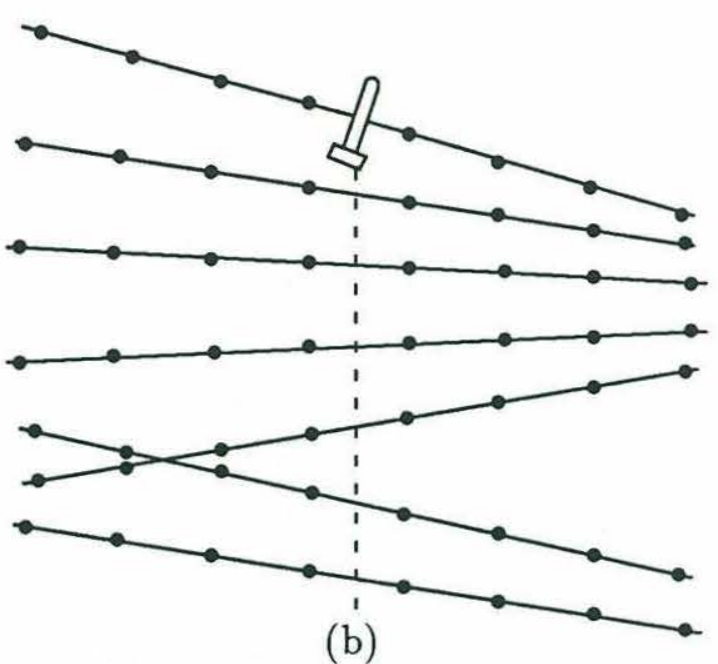

(b)

Figure 3.6: Effect of variations in speed and pitch angle (a) and yaw angle (b) on the sampling pattern, according to the linear model derived in Section 2.2.3.

resulting from the estimation error. However, at a closer look, some horizontal patterns are perceived which can be attributed to lateral displacement of the towfish. There are also other patterns that clearly result from linear objects in the original sonograph, such as the cables and scours on the bottom.

\subsection{Measuring Distortion in the Along-Track Di- rection}

In the along-track $(n)$ direction, distortions are caused by variations in the towfish speed and by pitching and yawing, which affect the sampling interval on the bottom by disturbing the constant pace at which the sonar beam would ideally move. Figure 3.6 illustrates the effect of variations of these attitude parameters on the sampling pattern on the bottom, again under the assumptions discussed at the beginning of Section 3.2. The resulting image looks "stretched" in areas where the sampling intervals in the alongtrack direction are smaller and "compressed" in areas where the sampling intervals are longer. A similar effect is observed in the shape of the local autocorrelation sequence of the image, as indicated in Fig. 3.7(a). The width of the main peak of the local sample 


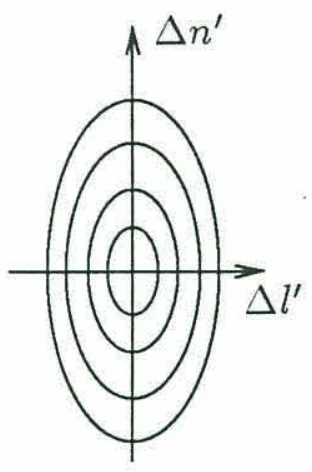

Stretched

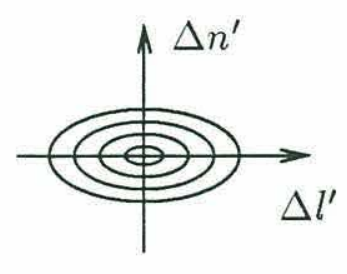

Compressed

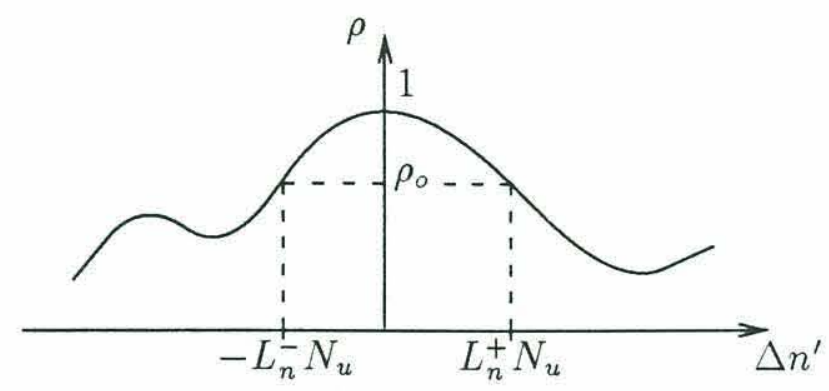

(b)

Figure 3.7: Along-track distortions cause local stretching or compressing of the autocorrelation sequence of the image in the $n$ direction (a). The distortions are reflected in the width of the main peak of the sample cross-correlation sequence $\rho$ at a cross-track lag $\Delta l^{\prime}=0$, and can be measured through the positive- and negative-lag correlation lengths, $L_{n}^{+}$and $L_{n}^{-}(\mathrm{b})$.

cross-correlation sequence at $\Delta l^{\prime}=0$ reflects the distortions in the image by becoming narrower or wider in different areas of the image. The width of the main peak can be measured through the correlation lengths $L_{n}^{+}[l, n]$ and $L_{n}^{-}[l, n]$, which are defined to be the positive and negative lags, respectively, at which the local sample cross-correlation sequence $\rho\left[0, \Delta n^{\prime} ; l N_{u}, n N_{u}\right]$ falls to a given threshold $\rho_{o}$, divided by the upsampling factor, $N_{u}$. Figure 3.7(b) illustrates the definition of the correlation lengths. Thus, we have

$$
\begin{aligned}
\rho\left[0, L_{n}^{+}[l, n] N_{u} ; l N_{u}, n N_{u}\right] & =\rho_{o} \\
\rho\left[0,-L_{n}^{-}[l, n] N_{u} ; l N_{u}, n N_{u}\right] & =\rho_{\circ} .
\end{aligned}
$$

Figure 3.8 shows enlargements of three regions of the sonograph in Figure 1.2, each one of size $31 \times 31$ pixels, along with the corresponding cross-correlation sequences at the center point and for $\Delta l^{\prime}=0$, and the correlation lengths in each case. They were calculated for a window seven pixels wide $(L=3)$ and a threshold $\rho_{0}$ of 0.5 . The first segment comes from an area of the image where there is no perceptible geometric distortion, whereas the other two exhibit noticeable stretching and compression in the 
along-track direction.

\subsubsection{Relation Between Correlation Lengths and Sampling Dis- placements}

In Section 3.2, we saw that it is straightforward to convert the location of the main peak of the sample cross-correlation sequence into an estimate of the sampling displacement $\Delta_{n} x_{s}^{\prime}[l, n]$. In fact, it is only necessary to multiply the lag at which the peak is located by the factor $-\left(c T_{s} / 2 N_{u}\right)$, as seen in Fig. 3.3. In the along-track direction, a complication arises because of the fact that the width of the main peak of the sample cross-correlation function is not directly related to the sampling displacement $\Delta_{n} y_{s}^{\prime}[l, n]$ that we seek to estimate, but rather to the sampling interval,

$$
P_{n}[l, n] \triangleq \sqrt{\left(\Delta_{n} x_{s}^{\prime}[l, n]\right)^{2}+\left(\Delta_{n} y_{s}^{\prime}[l, n]\right)^{2}},
$$

as illustrated in Fig. 3.9. This problem will be addressed later in this section, but first we will consider how the correlation lengths $L_{n}^{+}[l, n]$ and $L_{n}^{-}[l, n]$ are related to the sampling interval $P_{n}[l, n]$.

It is reasonable to expect that the correlation lengths should be approximately inversely proportional to the sampling interval at each point of the image. Specifically, in areas where the sonar beam scanned the bottom more rapidly, with a consequent increase in sampling interval, the sonograph image appears compressed, with a corresponding decrease in correlation lengths. Conversely, in areas where the sampling interval was smaller the sonograph appears stretched and the correlation lengths should be longer.

Let us consider for now only the positive-lags, for the sake of simplicity. Let $L_{b}[l, n]$ denote the correlation length of the autocorrelation function of the seabed backscattering function $b(x, y)$ at point $\left(x_{s}[l, n], y_{s}[l, n]\right)$, defined in the same manner as $L_{n}^{+}[l, n]$ was defined for the interpolated sonograph image in the preceding section. Because the autocorrelation function of $b(x, y)$ is assumed to be isotropic, its correlation length equals $L_{b}[l, n]$ in all directions. $L_{n}^{+}[l, n]$ is the distance in sonograph coordinates that we have to travel from point $[l, n]$ in the positive $n$ direction before the sample cross-correlation sequence drops below the threshold $\rho_{o}$ (see Eq. (3.5a)). Similarly, $L_{b}[l, n]$ is the distance 

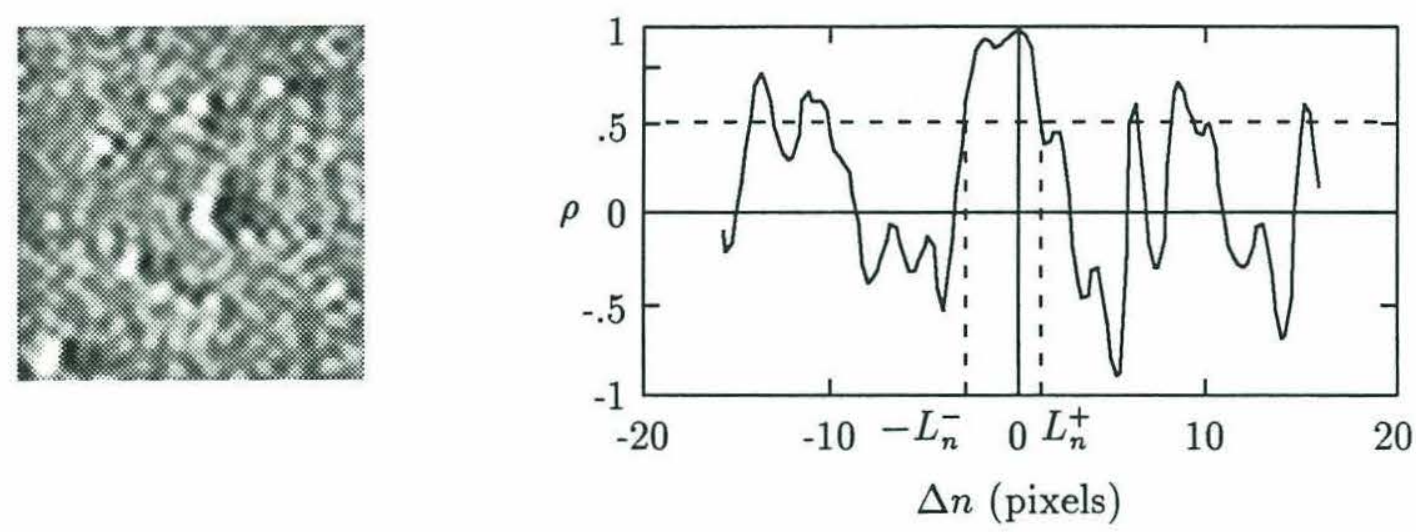

(a)
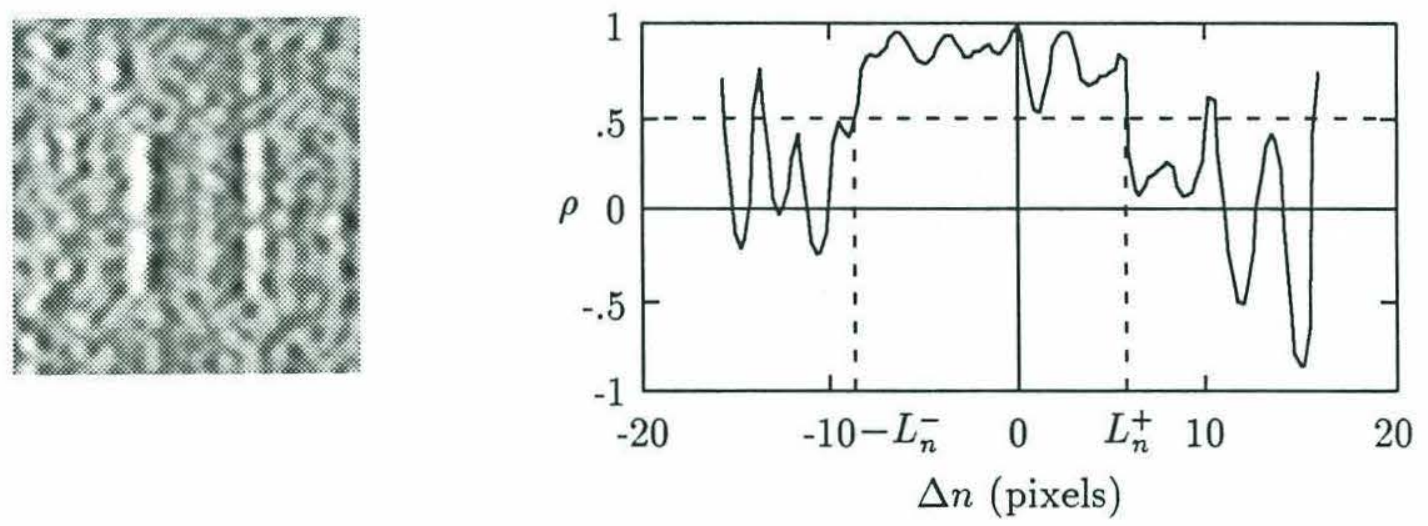

(b)
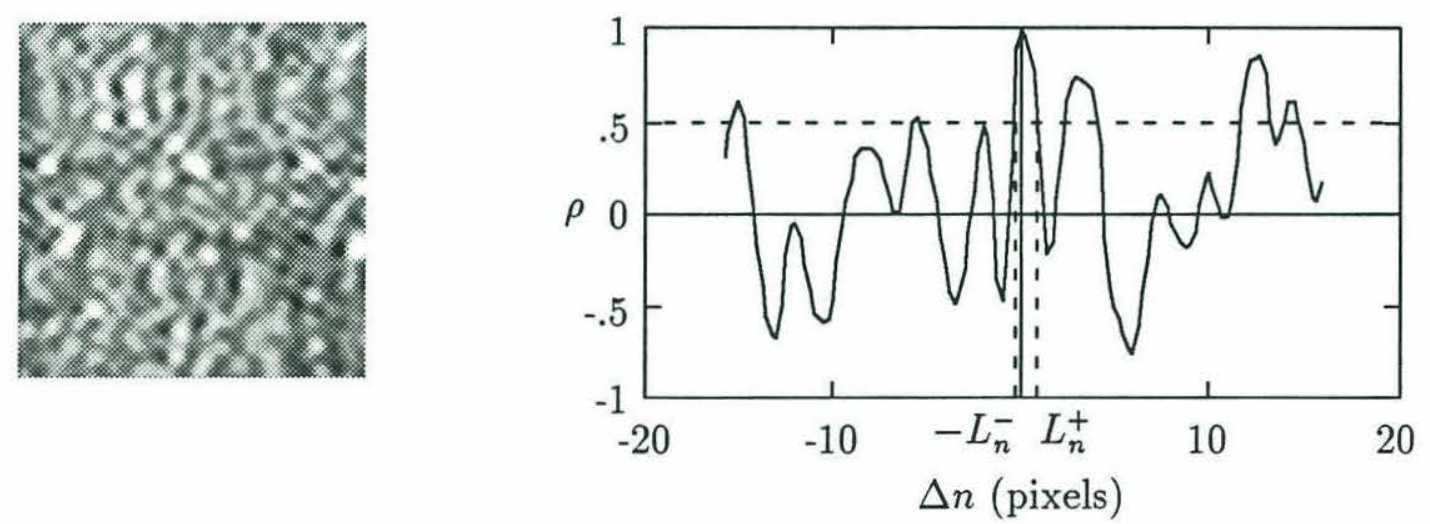

(c)

Figure 3.8: Examples of the effect of geometric distortions on the correlation length, for $15 \times 15$-pixel areas where the sampling rate was average $(a)$, greater than average $(b)$, and less than average (c). 


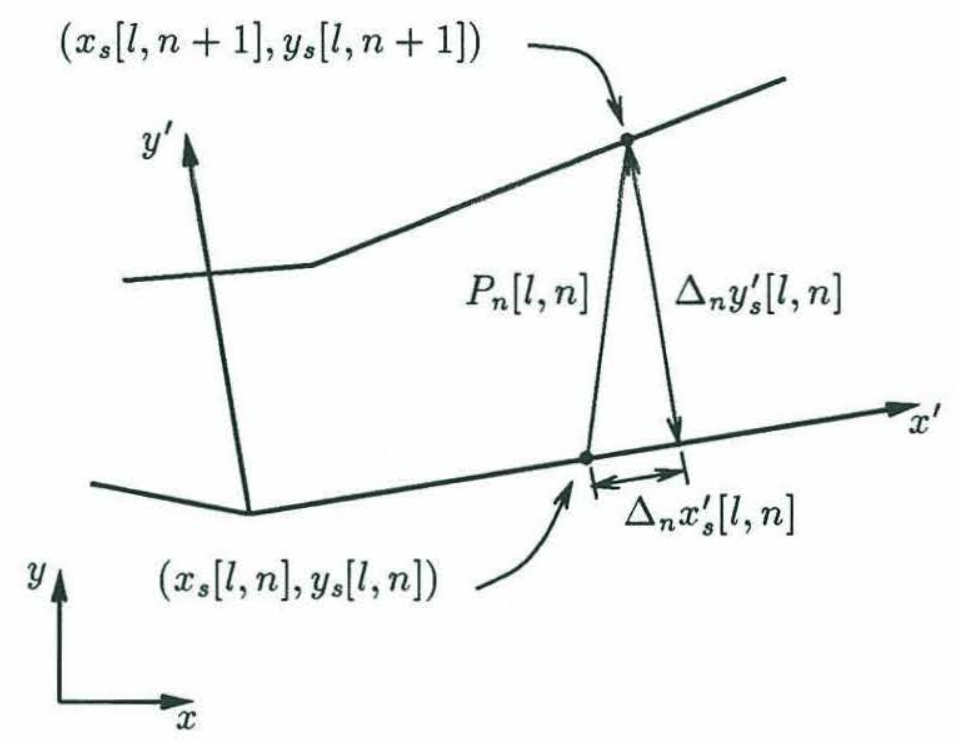

Figure 3.9: Relation between the sampling interval, $P_{n}[l, n]$, and the sampling displacements, $\Delta_{n} x_{s}^{\prime}[l, n]$ and $\Delta_{n} y_{s}^{\prime}[l, n]$.

the sonar beam has to travel on the seabed from point $\left(x_{s}[l, n], y_{s}[l, n]\right)$ before the autocorrelation function of $b(x, y)$ falls below that same threshold. Assuming for now that $L_{n}^{+}[l, n]$ is an integer number of pixels, one could say that $L_{b}[l, n]$ equals the sum of the $L_{n}^{+}[l, n]$ next sampling intervals $P_{n}[l, n]$ from point $\left(x_{s}[l, n], y_{s}[l, n]\right)$, or

$$
L_{b}[l, n]=\sum_{i=0}^{L_{n}^{+}[l, n]-1} P_{n}[l, n+i] .
$$

In general, however, the correlation length may not be an integer because, as explained in Section 3.1, the correlation coefficients are computed at non-integer lags so that $L_{n}^{+}[l, n]$ would not be subject to coarse quantization. Equation (3.6) can be modified to accommodate non-integer values of $L_{n}^{+}[l, n]$ by taking

$$
L_{b}[l, n]=\left(L_{n}^{+}[l, n]-\left\lfloor L_{n}^{+}[l, n]\right\rfloor\right) P_{n}\left[l, n+\left\lfloor L_{n}^{+}[l, n]\right\rfloor\right]+\sum_{i=0}^{\left\lfloor L_{n}^{+}[l, n]\right\rfloor-1} P_{n}[l, n+i],
$$

where $\left[L_{n}^{+}[l, n]\right\rfloor$ denotes $L_{n}^{+}[l, n]$ rounded towards zero.

Note that this equation may also be written as

$$
L_{b}[l, n]=L_{n}^{+}[l, n] \bar{P}_{n}^{+}[l, n]
$$


where $\bar{P}_{n}^{+}[l, n]$ represents the average of the $L_{n}^{+}[l, n]$ sampling intervals after point $[l, n]$. This shows that if $L_{b}[l, n]$ is constant, or at least fairly uniform throughout the sonograph, then, as expected, the positive-lag correlation length at point $[l, n]$ is inversely proportional to the sampling period, or, more accurately, to the average value of the sampling periods immediately after that point.

Now, let $L_{n o}[l]$ represent the average value of the correlation lengths in the absence of motion instabilities, i. e., with the towfish moving at a constant speed and with the heading aligned with the trajectory. ${ }^{3}$ No superscript is used in $L_{n o}[l]$ because it represents the averages of both $L_{n}^{+}[l, n]$ and $L_{n}^{-}[l, n]$, which should be equal if there is no geometric distortion. In the absence of motion instabilities the sampling periods equal $v T_{f}$, where $v$ is the speed of the deploying vessel, and Eq. 3.7 yields

$$
L_{b}[l, n]=v T_{f} L_{n o}[l]
$$

This allows us to rewrite that equation as

$$
v T_{f} L_{n o}[l]=\left(L_{n}^{+}[l, n]-\left\lfloor L_{n}^{+}[l, n]\right\rfloor\right) P_{n}\left[l, n+\left\lfloor L_{n}^{+}[l, n]\right\rfloor\right]+\sum_{i=0}^{\left\lfloor L_{n}^{+}[l, n]\right]-1} P_{n}[l, n+i] .
$$

This equation gives the relation between the sampling interval, $P_{n}[l, n]$ and the positive-lag correlation length, $L_{n}^{+}[l, n]$. Let us now consider how the correlation lengths may be related to the sampling displacements $\Delta_{n} y_{s}^{\prime}[l, n]$, the quantities we want to estimate. Recalling that $P_{n}[l, n]=\sqrt{\left(\Delta_{n} x_{s}^{\prime}[l, n]\right)^{2}+\left(\Delta_{n} y_{s}^{\prime}[l, n]\right)^{2}}$, and since $\Delta_{n} x_{s}^{\prime}[l, n]$ can be estimated separately as shown in the preceding section, we see that Eq. (3.8) may be used for estimating $\Delta_{n} y_{s}^{\prime}[l, n]$ from the correlation lengths $L_{n}^{+}[l, n]$, provided the value of $L_{n o}[l]$ is known. The estimation process would be complicated by the fact that this is a nonlinear equation. Notice, however, that if $\Delta_{n} x_{s}^{\prime}[l, n]$ is zero, then we have $P_{n}[l, n]=\left|\Delta_{n} y_{s}^{\prime}[l, n]\right|$ and $\mathrm{Eq}(3.8)$ becomes a linear equation relating the absolute value of $\Delta_{n} y_{s}^{\prime}[l, n]$ to $L_{n o}[l]$. This suggests that the task of estimating $\Delta_{n} y_{s}^{\prime}[l, n]$ can be considerably simplified if, before calculating the correlation lengths, we process the image to eliminate the distortions

\footnotetext{
${ }^{3}$ Strictly speaking, the average correlation length should be denoted $L_{n_{0}}[m]$, since the sonar beam pattern causes it to vary with range rather than with the horizontal distance on the bottom, as we will see in the next section. However, we denote it by $L_{n o}[l]$ for the sake of simplicity, with the conversion from $m$ to $l$ left implicit.
} 
in the cross-track direction, and thus eliminate the $\Delta_{n} x_{s}^{\prime}[l, n]$. That can be accomplished by shifting the lines of the image $\bar{s}[l, n]$ by the estimated values of the sampling displacements $\Delta_{n} x_{s}^{\prime}[l, n]$, thus obtaining a new image $\overline{\bar{s}}[l, n]$ for which $\Delta_{n} x_{s}^{\prime}[l, n]$ may be assumed to be zero. This procedure will be described in greater detail in Section 5.1. If the correlation lengths $L_{n}^{+}[l, n]$ are calculated as described before from the new image $\overline{\bar{s}}[l, n]$, instead of from $\bar{s}[l, n]$, then $\Delta_{n} x_{s}^{\prime}[l, n]$ can be set to zero in Eq. (3.8), yielding

$$
v T_{f} L_{n o}[l]=\left(L_{n}^{+}[l, n]-\left\lfloor L_{n}^{+}[l, n]\right\rfloor\right)\left|\Delta_{n} y_{s}^{\prime}\left[l, n+\left\lfloor L_{n}^{+}[l, n]\right\rfloor\right]\right|+\sum_{i=0}^{\left\lfloor L_{n}^{+}[l, n]\right]-1}\left|\Delta_{n} y_{s}^{\prime}[l, n+i]\right|,
$$

This is the desired expression relating the correlation lengths, $L_{n}^{+}[l, n]$, to the sampling displacements in the along-track direction, $\Delta_{n} y_{s}[l, n]$. If, for convenience, we define

$$
q[l, n] \triangleq \frac{\left|\Delta_{n} y_{s}^{\prime}[l, n]\right|}{v T_{f} L_{n o}[l]}
$$

then the last equation may also be written as

$$
\left(L_{n}^{+}[l, n]-\left\lfloor L_{n}^{+}[l, n]\right\rfloor\right) q\left[l, n+\left\lfloor L_{n}^{+}[l, n]\right]\right]+\sum_{i=0}^{L_{n}^{+}[l, n]-1} q[l, n+i]=1 .
$$

Similarly, for the negative-lag correlation lengths, we obtain

$$
\left(L_{n}^{-}[l, n]-\left\lfloor L_{n}^{-}[l, n]\right\rfloor\right) q\left[l, n+\left\lfloor L_{n}^{-}[l, n]\right]\right]+\sum_{i=0}^{L_{n}^{-}[l, n]-1} q[l, n+i]=1 .
$$

At each cross-track distance $l,(3.10 \mathrm{a})$ and $(3.10 \mathrm{~b})$ provide a set of $2\left(N_{n}-1\right)$ independent linear equations in the $\left(N_{n}-1\right)$ unknowns, ${ }^{4} q[l, n], n=0,1,2, \ldots, N_{n}-2$. These equations can then be used to find the deterministic least-squares estimates of those quantities from the correlation lengths $L_{n}^{+}[l, n]$ and $L_{n}^{-}[l, n]$ calculated from the image $\overline{\bar{s}}[l, n]$. If for each range index $l$ we define the vectors

$$
\left.q[l] \triangleq\left[\begin{array}{c}
q[l, 0] \\
q[l, 1] \\
\vdots \\
q\left[l, N_{n}-2\right]
\end{array}\right] \quad \text { and } \quad \underline{b} \triangleq\left[\begin{array}{c}
1 \\
1 \\
\vdots \\
1
\end{array}\right]\right\} 2\left(N_{n}-1\right)
$$

\footnotetext{
${ }^{4}$ There are only $\left(N_{n}-1\right)$ unknowns because we are dealing with sampling increments between lines of the image, and the number of increments is one less than the total number of lines, $N_{n}$.
} 
and form a matrix $D[l]$ with the coefficients of the $q[l, n]$ in Eqs. (3.10a) and (3.10b), then we may write

$$
D[l] \underline{q}[l]=\underline{b}[l]
$$

The estimates of $q[l, n], n=0,1, \ldots, N_{n}-1$ are finally obtained as the least-squares solution to this equation, i. e.,

$$
\hat{q}[l]=\left(D^{T}[l] D[l]\right)^{-1} D^{T}[l] \underline{b}[l]
$$

This procedure amounts to inverse filtering the $q[l, n]$ along each column of the sonograph, to compensate for the time-varying averaging implied by Eqs. (3.10a) and (3.10b)

We now have estimates of the quantities $q[l, n] \triangleq\left|\Delta_{n} y_{s}^{\prime}[l, n]\right| / v T_{f} L_{n o}[l]$. In order to obtain the desired estimates of the sampling displacements $\Delta_{n} y_{s}^{\prime}[l, n]$, it is necessary to know $L_{n o}[l]$, i. e., what the correlation lengths should be in the absence of geometric distortions. The next section will present a technique for its estimation.

\subsubsection{Correlation Lengths in the Absence of Geometric Dis- tortion}

The correlation lengths are affected by the composition of the seabed in the area scanned to form the sonograph. In fact, measures similar to the correlation lengths defined here have been used exactly for the purpose of classifying the different types of geological formations on the bottom, such as sand dunes, rock outcrops, mud, gravel, etc.[54] It is therefore necessary to know the correlation lengths associated with the different kinds of materials appearing in a sonograph, if one is to use variations in the correlation length as a measure of geometric distortion. This may require some sort of image segmentation process to divide the image in areas where the correlation lengths may be considered uniform. The sonograph we have been considering, however, presents fairly uniform topography and does not require such an operation.

The correlation lengths are also affected by small-scale variations caused by individual objects on the bottom, such as rocks, scours, cables, dunes, etc., and their acoustic shadows. What is important is that underneath these small-scale variations it is possible to 
perceive large-scale variations caused by geometric distortions, which will be used for estimating the towfish attitude parameters. The small-scale variations in correlation lengths translate into small-scale errors in the estimates of sampling displacements $\Delta_{n} y_{s}^{\prime}[l, n]$, which are incorporated as measurement noise in the model derived in Chapter 2.

As indicated in Chapter 1, the image intensity at a certain point of a sonograph ideally corresponds to the backscattering coefficient of the corresponding point on the seabed. Because of the sonar pulse envelope and beam spread, however, the image intensity at a given point is actually a weighted sum of the reflections from a small area around that point. Thus, a sonograph image in fact results from the convolution of the backscattering function of the seabed with the sonar beam pattern in the along-track direction and with the sonar pulse envelope in the cross-track direction. If the beam width changes as the wavefront propagates away from the sonar, there are corresponding changes in the sample cross-correlation sequence and, consequently, also in the correlation lengths. Figure 3.10 illustrates the effect of changes in beam width. The beam width is approximately equal to the length of the transducer array in the near field region and then starts to increase linearly with range in the far field, forming a wedge-shaped beam [11]. On average, the correlation lengths of the sonograph can be expected to exhibit a similar pattern.

We now need to estimate $L_{n o}[l]$ in order to obtain the final estimates of the sampling intervals $\Delta_{n} y_{s}^{\prime}[l, n]$. First we select a set $\mathcal{N}$ of lines from the sonograph where there is no backscanning and where the bottom morphology appears to be fairly uniform, so that $L_{b}[l, n]$ may be assumed constant and consequently the average correlation length may be a function of $l$ only. The lines should also come from areas of the sonograph where visual inspection reveals little or no geometric distortion, since $L_{n o}[l]$ is the correlation length in the absence of geometric distortions.

The average of the $q[l, n]$ over the $N_{\mathcal{N}}$ lines in the set $\mathcal{N}$ is ${ }^{5}$

$$
\frac{1}{N_{\mathcal{N}}} \sum_{n \in \mathcal{N}} q[l, n]=\frac{1}{N_{\mathcal{N}}} \sum_{n \in \mathcal{N}} \frac{\left|\Delta_{n} y_{s}^{\prime}[l, n]\right|}{v T_{f} L_{n o}[l]}
$$

\footnotetext{
${ }^{5}$ As previously noted, the average correlation length is actually a function of range $m$ rather than horizontal distance $l$. Therefore, the $q[l, n]$ should be added not for fixed $l$ but for values of $l$ corresponding to fixed values of $m$, according to the slant-range correction at each line. To avoid making the equations more complex, this modification is again left implicit.
} 


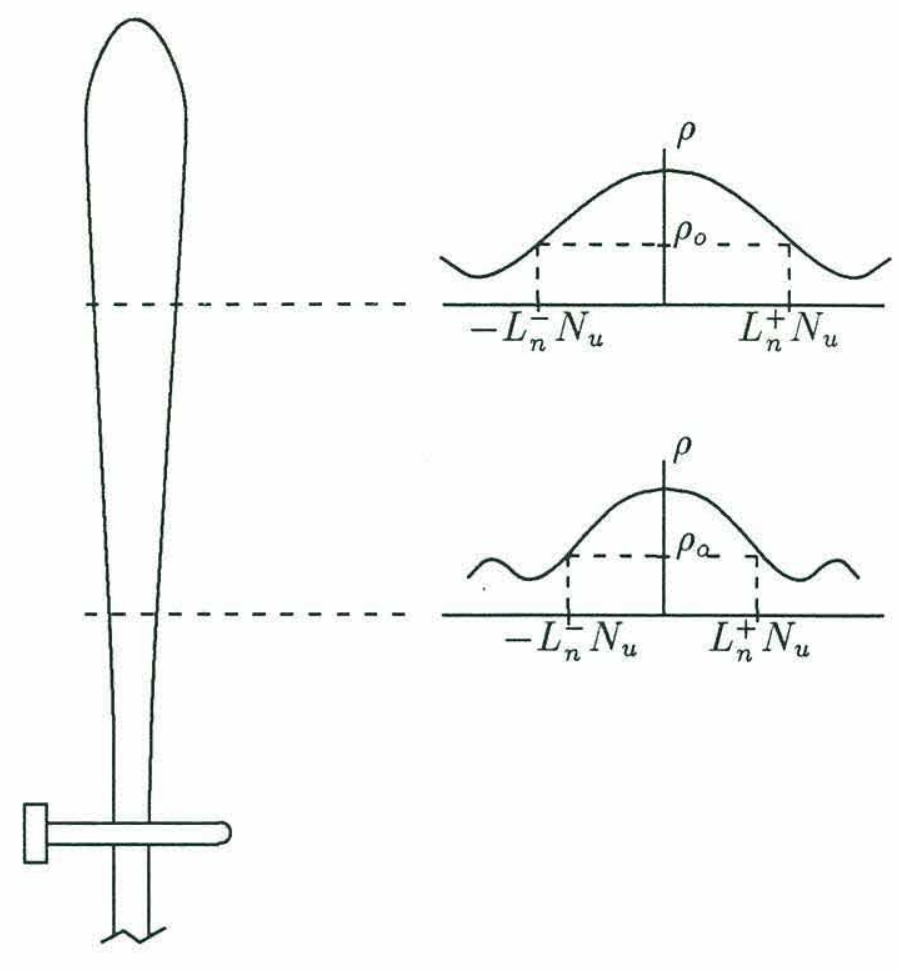

Figure 3.10: The widening of the sonar beam pattern with increasing range is responsible for a corresponding increase in the correlation lengths of the image.

$$
\begin{aligned}
& =\frac{1}{N_{\mathcal{N}} v T_{f} L_{n o}[l]} \sum_{n \in \mathcal{N}} \Delta_{n} y_{s}^{\prime}[l, n] \\
& =\frac{1}{N_{\mathcal{N}} v T_{f} L_{n o}[l]} \sum_{n \in \mathcal{N}}\left(\Delta y_{o}^{\prime}[n]+l\left(c T_{s} / 2\right) \Delta \theta[n]\right),
\end{aligned}
$$

where in the last step we used Eq. (2.12). If we symmetrically add the $q[l, n]$ for $l= \pm 1, \pm 2, \ldots, \pm\left(N_{l}-1\right)$, the yaw-dependent term disappears, yielding

$$
\frac{1}{2 N_{\mathcal{N}}} \sum_{n \in \mathcal{N}}(q[l, n]+q[-l, n])=\frac{1}{N_{\mathcal{N}} v T_{f} L_{n o}[l]} \sum_{n \in \mathcal{N}} \Delta y_{o}^{\prime}[n]
$$

We have thus reduced the problem to knowing the average value of only $\Delta y_{0}^{\prime}[n]$ (in the $\left(x^{\prime}, y^{\prime}\right)$ coordinate system) over the selected lines. In principle, this value is not known. However, the average value of $\Delta y_{o}[n]$ (in the $(x, y)$ system) over the entire sonograph should equal $v T_{f}$, the average distance traveled by the towing vessel in the $y$ direction 
between pulses. This assumes that at the end of the acquisition of the sonograph the distance between the towfish and the deploying vessel in the along-track direction is the same as in the beginning. We will use this assumption to calculate the average value of $\Delta y_{\sigma}^{\prime}[n]$ over the selected lines later on. For now we represent this average value by $v_{\mathcal{N}}^{\prime} T_{f}$, and incorporate it into $L_{n o}[l]$ as a scale factor. Thus the last equation yields

$$
\frac{v}{v_{\mathcal{N}}^{\prime}} L_{n o}[l]=\frac{1}{\frac{1}{2 N_{\mathcal{N}}} \sum_{n \in \mathcal{N}}(q[l, n]+q[-l, n])}
$$

Figure 3.11(a) shows the average value of $\hat{q}[l, n]$ for the sonograph we are considering, calculated from 51 lines (out of a total of 512 lines) that appear to present the least amount of geometric distortion. Since the effect of the beam pattern may be expected to vary smoothly, a fourth-order polynomial can be fitted to that average as seen in the figure. That polynomial constitutes our estimate of $\left(v / v_{\mathcal{N}}^{\prime}\right) \hat{L}_{n o}[l]$. The effect of the beam pattern is seen clearly in this curve, added to the constant level of the correlation length of the backscattering function. As expected, the curve is approximately constant in the near field portion of the beam and then increases linearly with range in the far field, before collapsing as a result of sound absorption by the water as we approach the maximum range of $100 \mathrm{~m}$. The dashed straight line shown in the figure was made to coincide with the approximately linear portion of the curve. By extrapolating it to a range of zero we can determine the value of the correlation length of the bottom (times the factor $\left.\left(v / v_{\mathcal{N}}^{\prime}\right)\right)$. The resulting value is $12.4 \mathrm{~cm}$. If we subtract that constant level from the polynomial and plot it twice "back-to-back" as in part (b) of the figure, we obtain a clear representation of the effect of the beam pattern on the correlation lengths.

The estimate $\hat{L}_{n o}[l]$ may now be multiplied by the estimates of $q[l, n]$ yielding estimates of the scaled sampling displacements $\left(v / v_{\mathcal{N}}^{\prime}\right)\left|\Delta_{n} y_{s}^{\prime}[l, n]\right|$,

$$
\frac{v}{v_{\mathcal{N}}^{\prime}}\left|\widehat{\Delta_{n} y_{s}^{\prime}}[l, n]\right|=\frac{v}{v_{\mathcal{N}}^{\prime}} \hat{L}_{n o}[l] \hat{q}[l, n]
$$

When we apply the linear model of Eq. (2.12) to these estimates of the sampling displacement, the resulting estimates of $\Delta y_{o}^{\prime}[n]$ and $\Delta \theta[n]$ reflect the scaling factor $\left(v / v_{\mathcal{N}}^{\prime}\right)$. Then we can finally calculate the value of $\left(v / v_{\mathcal{N}}^{\prime}\right)$ by requiring that the average value of 


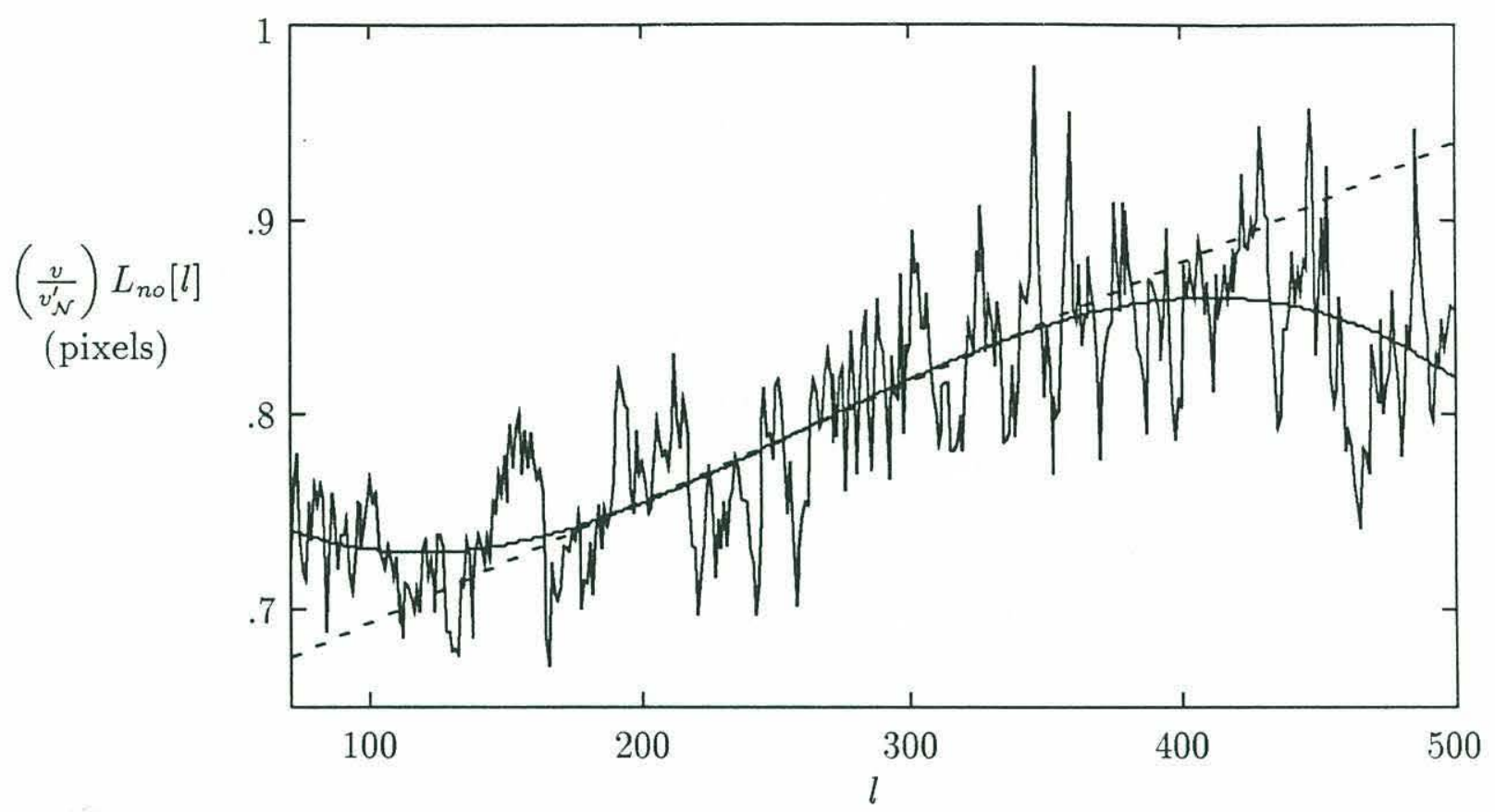

(a)

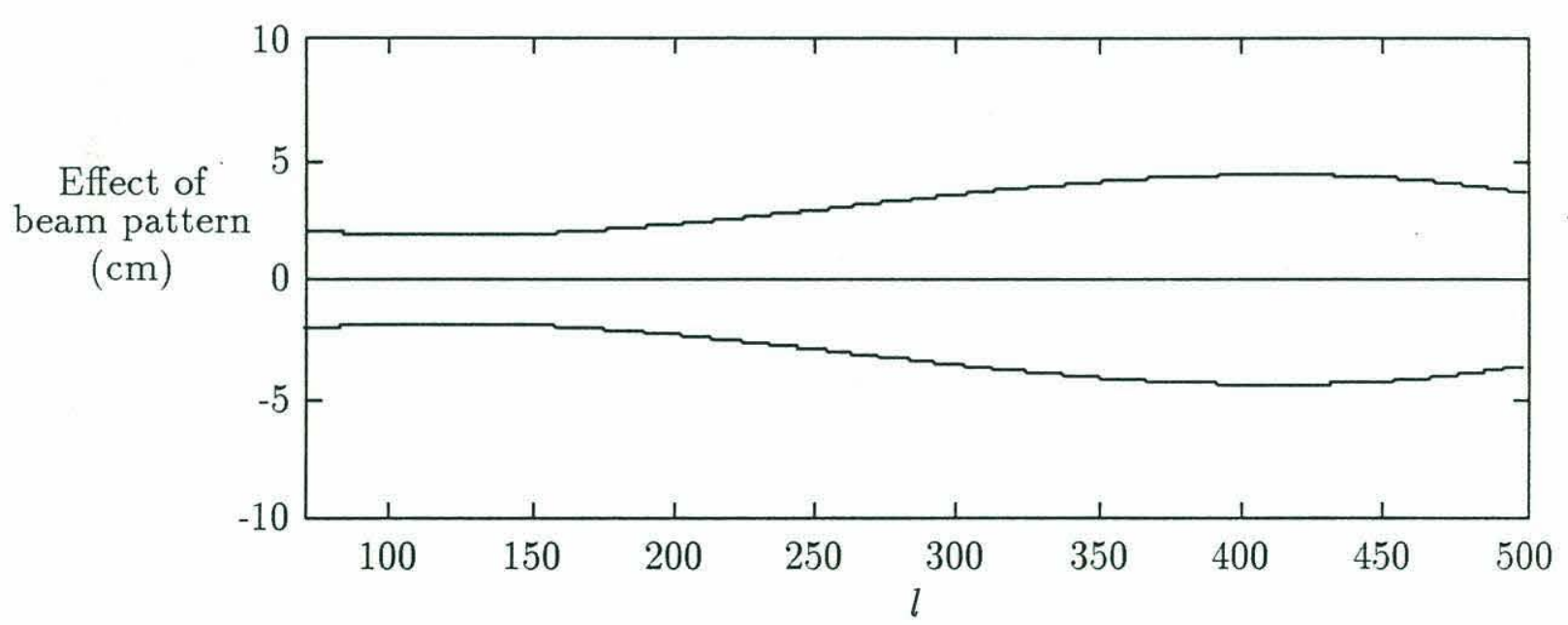

(b)

Figure 3.11: Estimate of $\left(v / v_{\mathcal{N}}^{\prime}\right) L_{n o}[l]$ obtained by averaging the correlation lengths for a selected set of lines of the sonograph (a). The increment in correlation lengths due to the effect of the beam pattern is represented by the curves in (b), which have the shape that could be expected for the beam pattern of a linear array. 
$\Delta y_{o}[n]$ over the entire sonograph be equal to $v T_{f}$. To keep the notation simple, we do not modify the equations of Chapter 2 to incorporate the scaling factor $\left(v / v_{\mathcal{N}}^{\prime}\right)$. Rather, we ignore its existence for now and simply rescale the parameter estimates obtained in Chapter 4 so that the mean value of $\Delta y_{o}[n]$ over the entire sonograph equals $v T_{f}$.

Figure 3.12 illustrates the complete process of estimating the sampling displacements, and corresponds to one of the blocks of the overall scheme shown in Fig. 2.7. Fig. 3.13 shows the estimates $\left|\widehat{\Delta_{n} y_{s}^{\prime}}[l, n]\right|$ for our sample sonograph, scaled by the as yet unknown factor, $\left(v / v_{\mathcal{N}}^{\prime}\right)$. The image intensity at each point is proportional to $\left(v / v_{\mathcal{N}}^{\prime}\right)\left|\widehat{\Delta_{n} y_{s}^{\prime}}[l, n]\right|$ according to the tone scale shown below the image.

\subsubsection{Detection of Backscanning}

The last problem to address is that we have obtained estimates not of the sampling displacements $\Delta_{n} y_{s}^{\prime}[l, n]$, but of their absolute values. Therefore, it is necessary to detect areas where $\Delta_{n} y_{s}^{\prime}[l, n]$ is negative, i. e., areas where backscanning occurs, and multiply our estimates by minus one in those areas. One possible way of detecting backscanning is to look for areas where objects appear in triplicate. A significant problem with that approach is that it is not always easy to identify these triple images because each one of them is made from a different viewing angle and may differ considerably from the others. Fortunately, there is a more practical alternative based on looking for indications of backscanning in the estimates of $\Delta_{n} y_{s}^{\prime}[l, n]$ themselves. If, for instance, the sampling intervals along one line of the sonograph decrease with range until they become very small and then start to increase again, then the portion of the line after the minimum was probably backscanned as a result of yawing. Visual inspection of the suspected area can confirm whether backscanning really occurred or whether we have a false alarm.

Figure 3.14 shows the sampling interval in the along-track direction for a line of the sonograph presenting backscanning caused by yawing. Since the measured sampling interval is typically very noisy, it was found useful to fit a third-order polynomial to the curve in order to help determine the minimum point, as shown in the figure. A simple rule that yields good results is to decide that backscanning occurred if the minimum value of the polynomial falls below a threshold chosen according to some given criterion. 


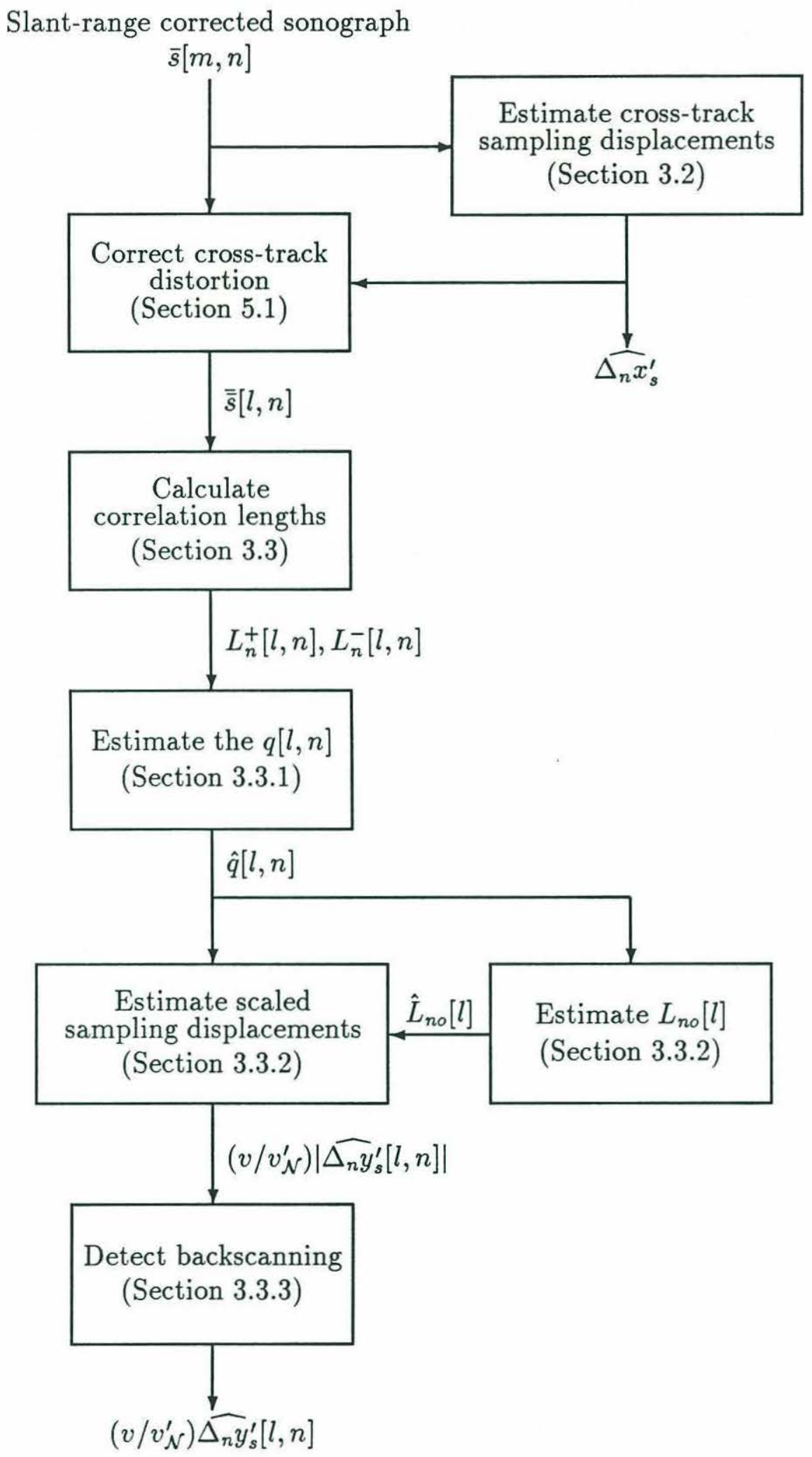

Figure 3.12: Overall technique for estimating sampling displacements. 

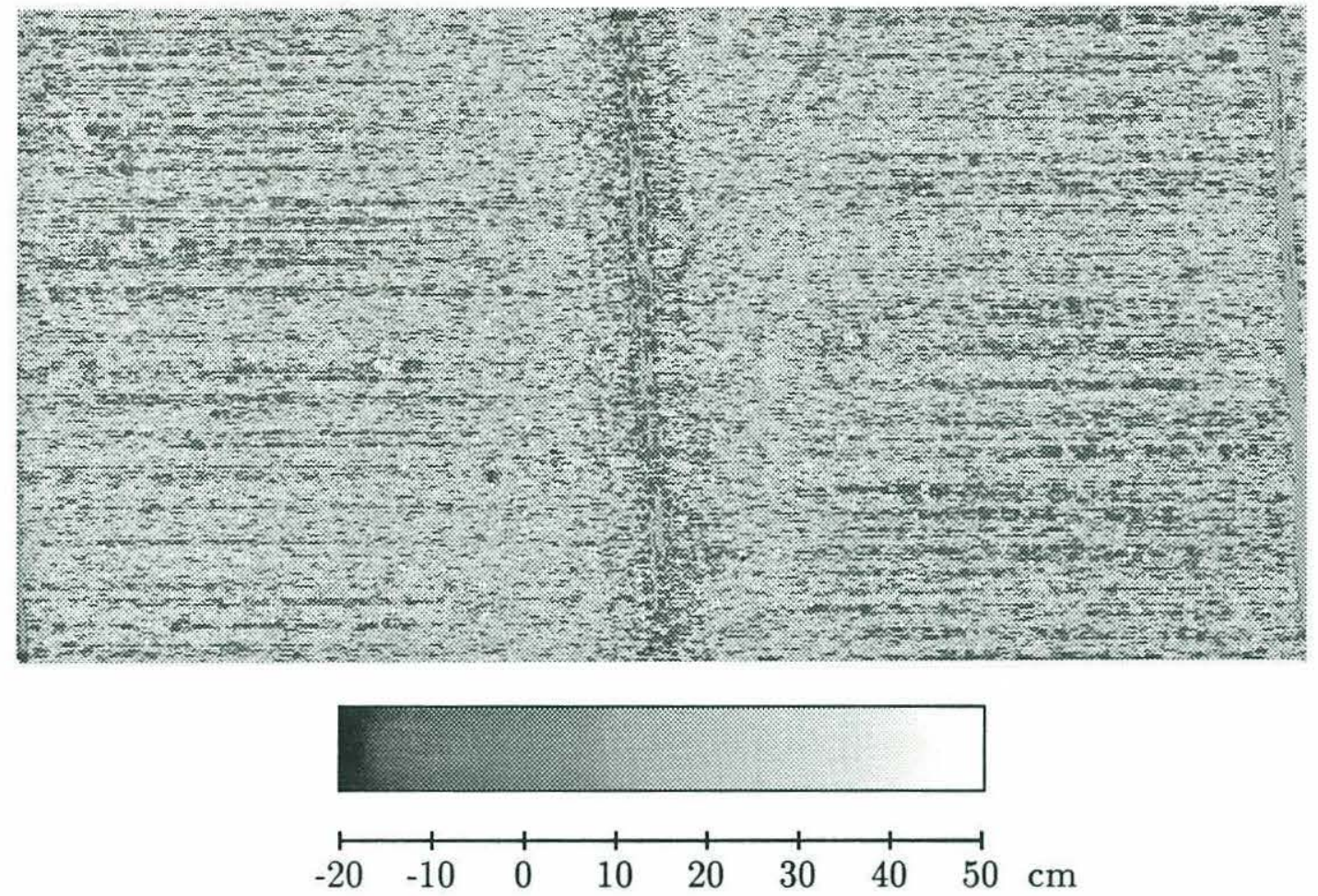

Figure 3.13: Estimates of $\left(v / v_{\mathcal{N}}^{\prime}\right)\left|\Delta_{n} y_{s}^{\prime}[l, n]\right|$ for the sonograph of Fig. 3.4. The image intensity at each point is proportional to $\left(v / v_{\mathcal{N}}^{\prime}\right)\left|\widehat{\Delta_{n} y_{s}^{\prime}}[l, n]\right|$ at that point, according to the tone scale shown above.

For instance, the threshold may be chosen so that the number of false alarms and misses is approximately the same. In our case, a threshold of 0.58 pixel or approximately $11 \mathrm{~cm}$ satisfies that criterion. As for pitch-induced backscanning, if we find two neighboring groups of lines of the sonograph where the sampling interval is very small on both the starboard and the port side, it is possible that the area between these two groups of lines was backscanned as a result of pitching. Again, visual inspection of the suspected strip can indicate whether backscanning actually occurred.

Figure 3.15(a) shows the areas in the sonograph where backscanning was detected, as estimated through this technique. Visual inspection of the original sonograph reveals 40 false alarms and 40 misses out of the total 512 lines (less than $8 \%$ in each case). Notice that in this sonograph the backscanned areas alternate between the starboard and port sides, indicating that the backscanning is due to yawing. Part (b) of the figure shows an enlargement of the lower right-hand corner of the original image for closer inspection of 


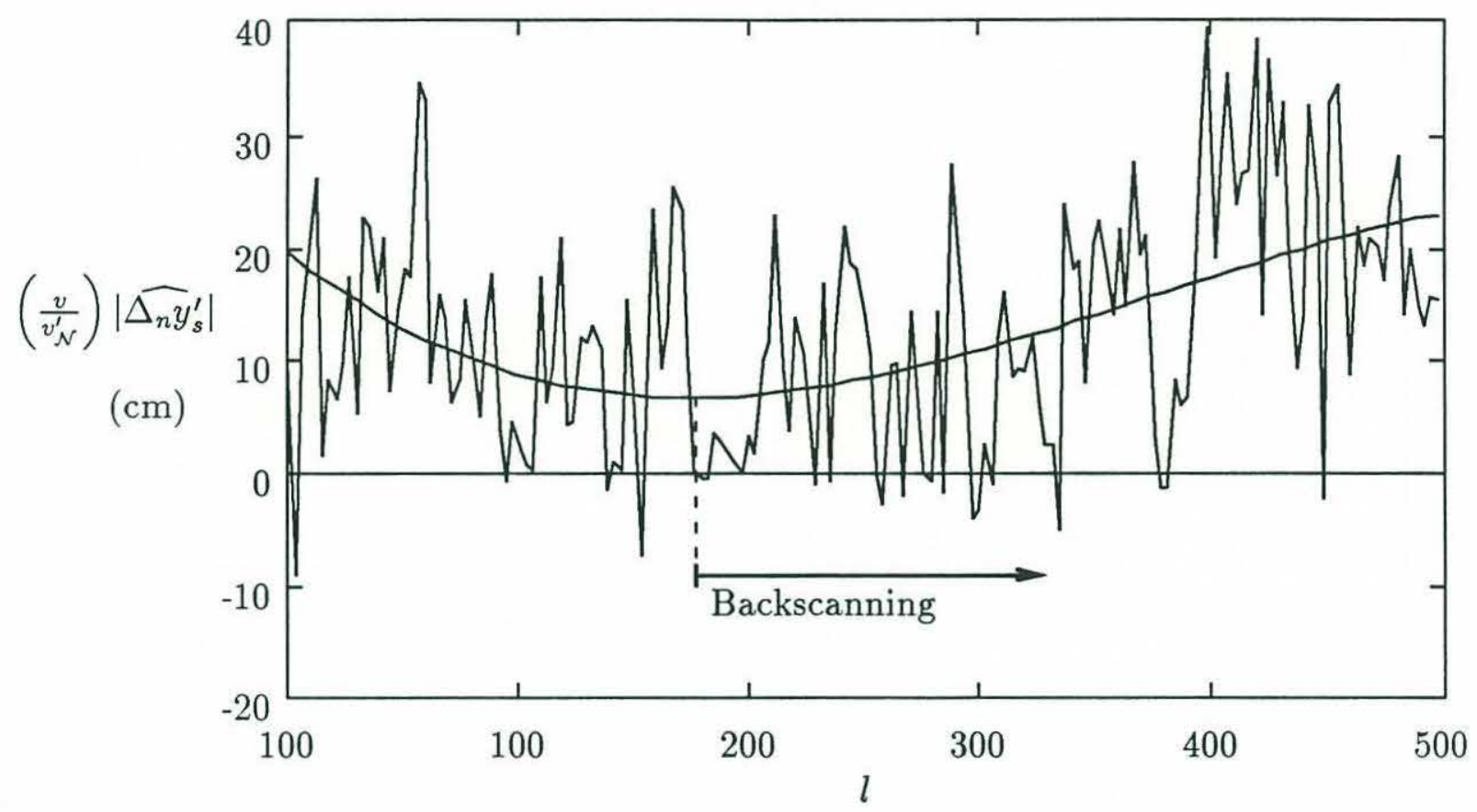

Figure 3.14: Backscanning induced by yawing can be detected by looking for lines where the estimates $\left|\Delta_{n} y_{s}^{\prime}[l, n]\right|$ decrease to approximately zero and than start increasing again, as shown in this example. A third-order polynomial is used to help locate the point where backscanning begins.

the backscanned areas. The presence of a cable on the bottom in that area provides a way to verify the accuracy of the technique for detecting backscanning. In fact, the lines where the cable appears to abruptly change direction were subject to backscanning, since in all likelihood it ran smoothly on the bottom towards the lower right-hand corner of the image. Comparing the original image with the image where backscanning is indicated, we see that the algorithm is very accurate in selecting the lines where the cable appears to change direction.

The last step in the estimation of sampling displacements is to multiply $\left|\widehat{\Delta_{n} y_{s}^{\prime}}[l, n]\right|$ by -1 in the areas where backscanning occurs. We thus obtain the estimates $\widehat{\Delta_{n} y_{s}^{\prime}}[l, n]$, which, along with the estimates $\widehat{\Delta_{n} x_{s}^{\prime}}[l, n]$ obtained in Section 3.2, are used in the next chapter for the estimation of the distortion parameters $\Delta x_{o}^{\prime}[n], \Delta y_{o}^{\prime}[n]$ and $\Delta \theta[n]$. 


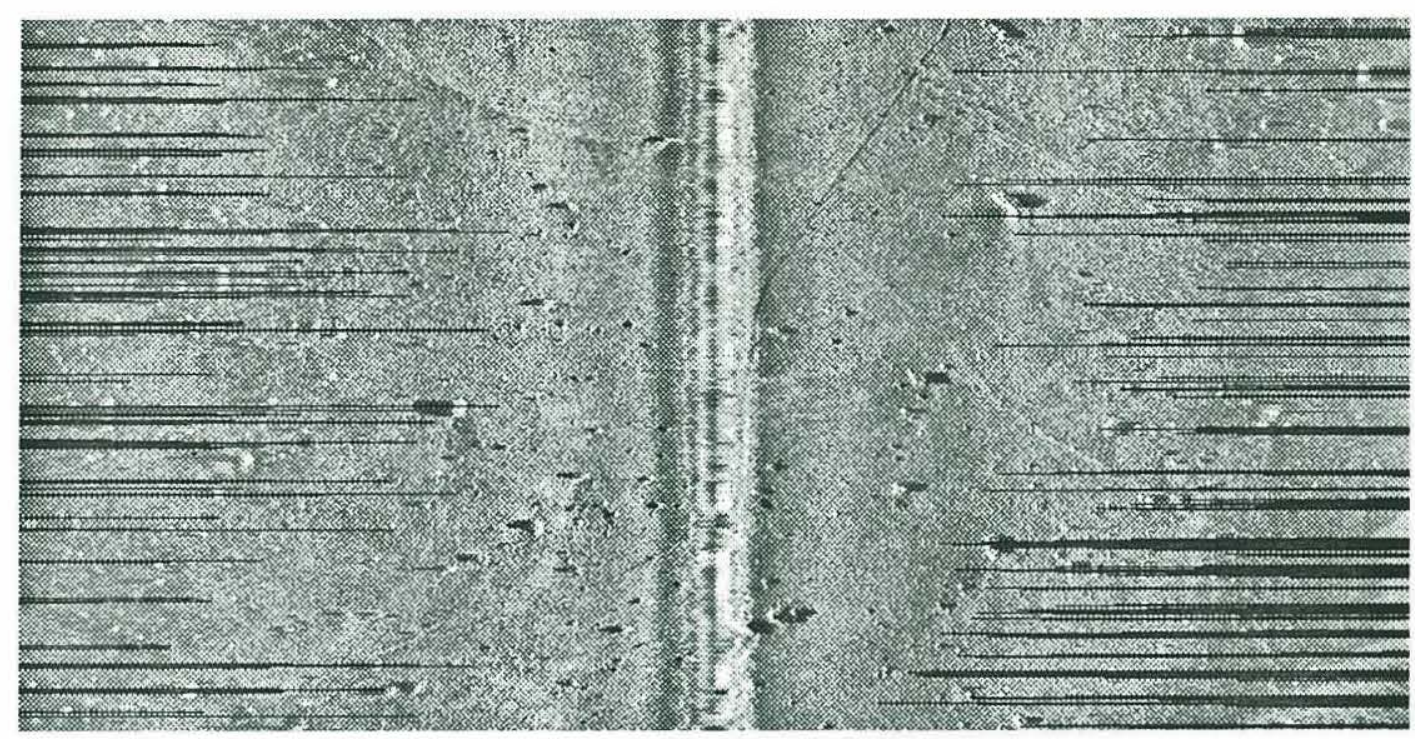

(a)
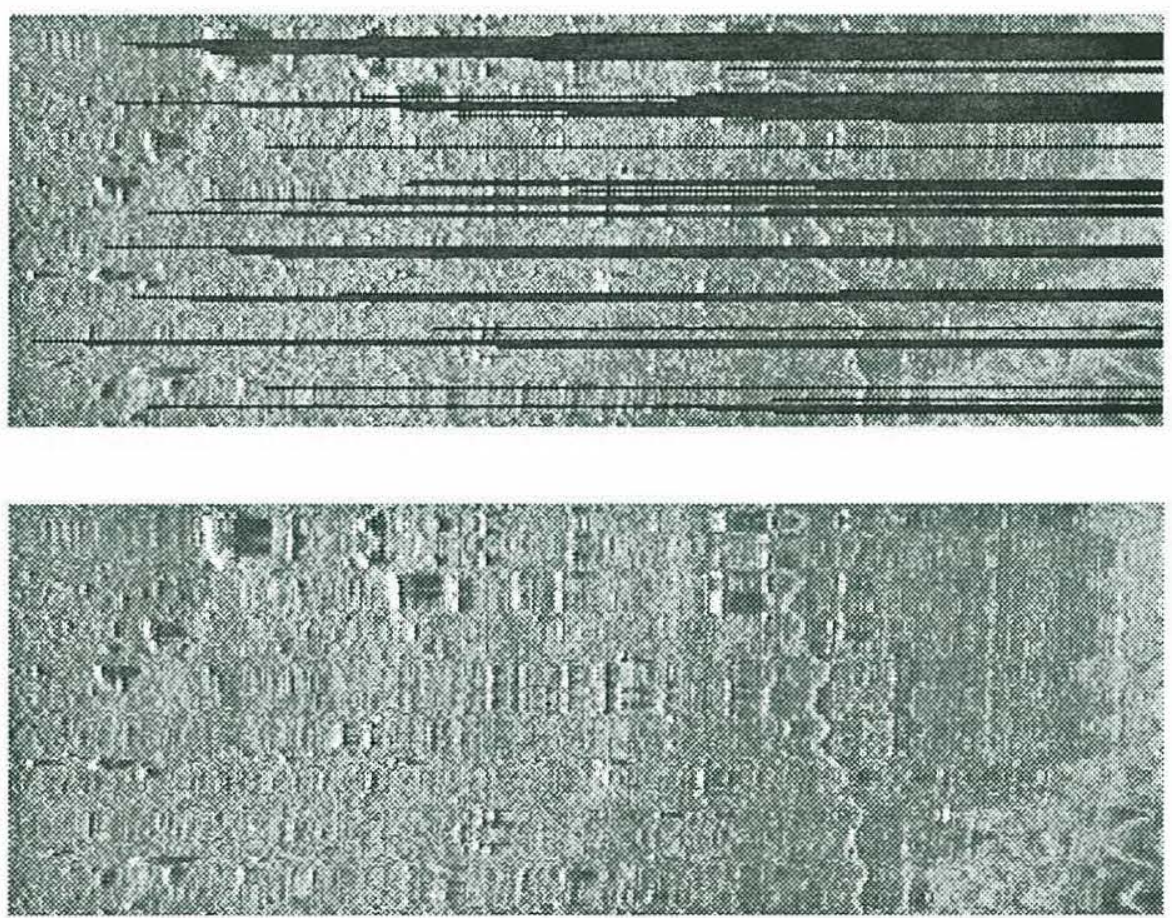

(b)

Figure 3.15: Areas where backscanning was detected are shown in black. In figure (a) these areas are seen to alternate between the starboard and port sides, indicating that backscanning in this sonograph was primarily caused by yawing. In (b) the lower righthand corner of the image is enlarged for comparison with the original image. 


\section{Chapter 4}

\section{Estimation of Distortion}

\section{Parameters}

In Chapter 3 we saw how the sampling displacements $\Delta_{n} x_{s}^{\prime}[l, n]$ and $\Delta_{n} y_{s}^{\prime}[l, n]$ can be estimated from the sonograph. We now consider how from those estimates we may in turn estimate the distortion parameters $\Delta x_{\circ}^{\prime}[n], \Delta y_{o}^{\prime}[n]$ and $\Delta \theta[n]$ of the linear model derived in Chapter 2. As explained in Section 2.2.2, by applying a lower-dimensional model to the estimates of sampling displacements, we may expect a reduction in their estimation error. As shown in the overall scheme presented in Fig. 2.7, we will then be ready to estimate the sampling coordinates of each point of the sonograph and proceed to rectify the geometric distortions in Chapter 5 .

\subsection{Selection of Observation Points}

We now seek to estimate the distortion parameters $\Delta x_{o}^{\prime}[n], \Delta y_{o}^{\prime}[n]$ and $\Delta \theta[n]$ from the estimates of $\Delta_{n} x_{s}^{\prime}[l, n]$ and $\Delta_{n} y_{s}^{\prime}[l, n]$ obtained in Chapter 3. The estimation is carried out on a set of distances $\mathcal{L}=\left\{l_{1}, l_{2}, \ldots, l_{p}\right\}$. In principle, this set could contain all columns of the sonograph from both the starboard and port sides, i. e., $\mathcal{L}=$ $\left\{-N_{l}, \ldots,-1,0,1, \ldots,\left(N_{l}-1\right)\right\}$. However, it has been found advantageous to attempt to render the estimation process more robust by choosing a subset of the columns for which the estimates of sampling displacements are judged more reliable according to 
some criterion. For instance, if visual inspection of the sonograph reveals areas where the correlation lengths may vary markedly because of the local morphology, or because of artifacts in the image such as acoustic shadows, then the selected set of distances should be adjusted, possibly on a line-by-line basis, to leave out those areas. We will therefore represent the selected distances for the $n$th line by $\mathcal{L}[n]$. As a general rule, we have found it to be advisable to omit the first few columns on each side of the bottom track (usually about $10 \%$ of the total number), since in that area the estimates of sampling displacements tend to be affected by the undersampling resulting from the slant-range distortion. That effect can be clearly seen in the stripe running vertically in the middle of Fig. 3.13, which displayed the estimates of $\Delta_{n} y_{s}^{\prime}[l, n]$.

Whenever the estimates of sampling displacements reveal pronounced yawing of the towfish, as in the sonograph we have been considering, it is also advisable to change $\mathcal{L}[n]$ from line to line so that the selected columns always come from the side towards which the towfish was turning at the time that line was acquired. In fact, yawing causes the beam to sweep the bottom faster on the other side, which may result in undersampling and a consequent loss of accuracy in the estimates of sampling displacements. Figure 4.1 illustrates this problem by presenting the estimates of $\Delta_{n} y_{s}^{\prime}[l, n]$ for a line of the sonograph in which the towfish turned towards the starboard side. The straight line shown in the figure is the result of fitting the linear model of Eq. (2.14b) to the estimates of $\Delta_{n} y_{s}^{\prime}[l, n]$ on the starboard side $(l>0)$. A good fit is obtained on that side, but on the port side the estimates of $\Delta_{n} y_{s}^{\prime}[l, n]$ depart from the model because of undersampling. Therefore, applying the linear model to both sides would result in less accurate estimates of the distortion parameters. An estimate of which side the towfish was turning towards for each line of the sonograph is easily obtained by computing the average value of $\widehat{\Delta_{n} y_{s}^{\prime}}[l, n]$ on the starboard and port sides of the line. The side with the smallest average sampling displacement is the side the towfish was turning towards, and the columns for $\mathcal{L}[n]$ are chosen from there.

For the estimations presented in the next sections we selected every fourth column from columns number 76 to 475 on the side with the smallest average sampling displace- 


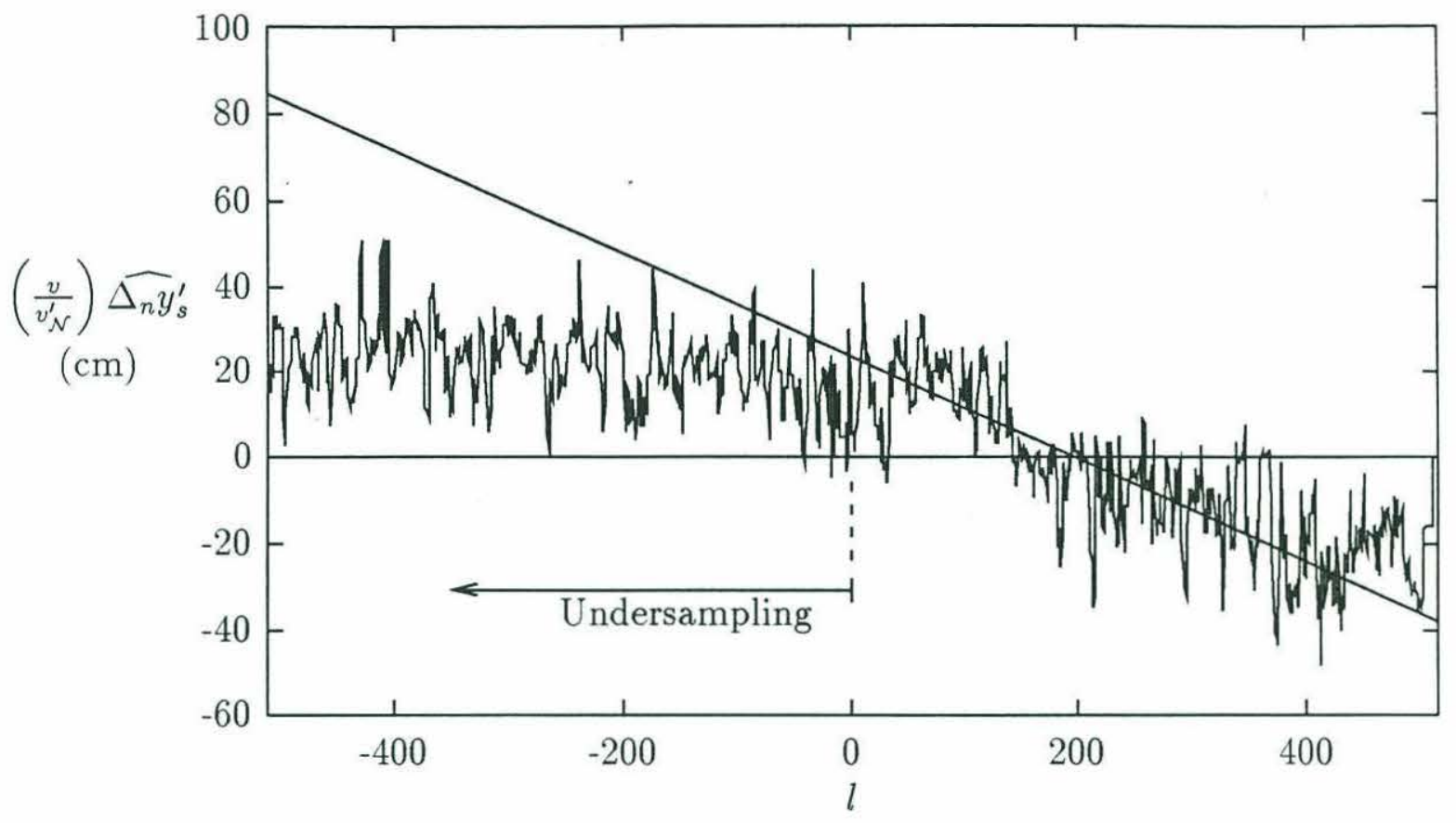

Figure 4.1: Example of a line affected by undersampling. The straight line is obtained by fitting the linear model to the estimates of sampling displacements on the starboard side $(l>0)$. Because of undersampling, the estimates of sampling displacements on the port side $(l<0)$ are smaller than what the linear model indicates they should be.

ment. Thus

$$
\mathcal{L}[n]= \begin{cases}\{76,80, \ldots, 472,475\}, & \text { if } \sum_{l=76}^{475} \widehat{\Delta_{n} y_{s}^{\prime}}[l, n]<\sum_{l=76}^{475} \widehat{\Delta_{n} y_{s}^{\prime}}[-l, n], \\ \{-76,-80, \ldots,-472,-475\}, & \text { otherwise. }\end{cases}
$$

The number of observations ( $p=100)$ was limited by the resulting memory requirements and execution times of the algorithms. In principle, better results might be obtained with a greater number of observations.

Figure 4.2 illustrates the technique for estimating the distortion parameters, and is part of the overall scheme presented in Fig. 2.7. For each line of the sonograph a set of distances $\mathcal{L}[n]$ is first chosen, as described above. The distortion parameters for that line are then estimated from the estimates of sampling displacements, $\widehat{\Delta_{n} x_{s}^{\prime}}[l, n]$ and $\widehat{\Delta_{n} y_{s}^{\prime}}[l, n]$, at $l \in \mathcal{L}[n]$ through one of two techniques described in the next sections. After all lines of the sonograph have been processed, the resulting estimates of $\Delta y_{0}[n]$ are then scaled 
so that their average value equals one, thus compensating for the scaling factor $\left(v / v_{\mathcal{N}}^{\prime}\right)$ introduced in Section 3.3.2. The estimates of $\Delta x_{o}^{\prime}[n]$ and $\Delta y_{o}^{\prime}[n]$ are then recalculated to reflect the scaling. Finally, the estimates of attitude parameters, $\hat{x}_{f}[n], \hat{\phi}[n]$ and $\hat{\theta}[n]$, may be calculated, though that is not required for the correction of geometric distortions, as indicated in Chapter 2. These steps will be examined in detail in the next sections.

\subsection{Deterministic Least-Squares Estimation}

One way of estimating the distortion parameters and attitude parameters is to do it separately for each line of the sonograph using deterministic least-squares estimation. This approach may be used in the absence of information about the statistics of the parameters and observation noise.

\subsubsection{Estimating the Distortion Parameters}

The model used for estimating the distortion parameters is

$$
\begin{aligned}
\widehat{\Delta_{n} x_{s}^{\prime}}[l, n] & =\Delta x_{o}^{\prime}[n]+v_{x}[l, n] \\
\widehat{\Delta_{n} y_{s}^{\prime}}[l, n] & =\Delta y_{o}^{\prime}[n]+l\left(c T_{s} / 2\right) \Delta \theta[n]+v_{y}[l, n],
\end{aligned}
$$

which corresponds to Eq. (2.12a) and (2.12b) with additional noise terms, $v_{x}[l, n]$ and $v_{y}[l, n]$, that represent the error incurred in replacing $\Delta_{n} x_{s}^{\prime}[l, n]$ and $\Delta_{n} y_{s}^{\prime}[l, n]$ with their estimates.

Employing deterministic least-squares approximation amounts to choosing $\widehat{\Delta x_{o}^{\prime}}[n]$, $\widehat{\Delta y_{0}^{\prime}}[n]$, and $\widehat{\Delta \theta}[n]$ so as to minimize the cost functions

$$
\begin{aligned}
& \varepsilon_{x}^{2}[n] \triangleq \sum_{l \in \mathcal{L}[n]}\left(\widehat{\Delta_{n} x_{s}^{\prime}}[l, n]-\widehat{\Delta x_{o}^{\prime}}[n]\right)^{2} \\
& \varepsilon_{y}^{2}[n] \triangleq \sum_{l \in \mathcal{L}[n]}\left(\widehat{\Delta_{n} y_{s}^{\prime}}[l, n]-\widehat{\Delta y_{o}^{\prime}}[n]-l\left(c T_{s} / 2\right) \widehat{\Delta \theta}[n]\right)^{2} .
\end{aligned}
$$

If $p[n]$ denotes the number of observation points in $\mathcal{L}[n]$, and if we define

$$
S_{l}[n] \triangleq \sum_{l \in \mathcal{L}[n]} l
$$




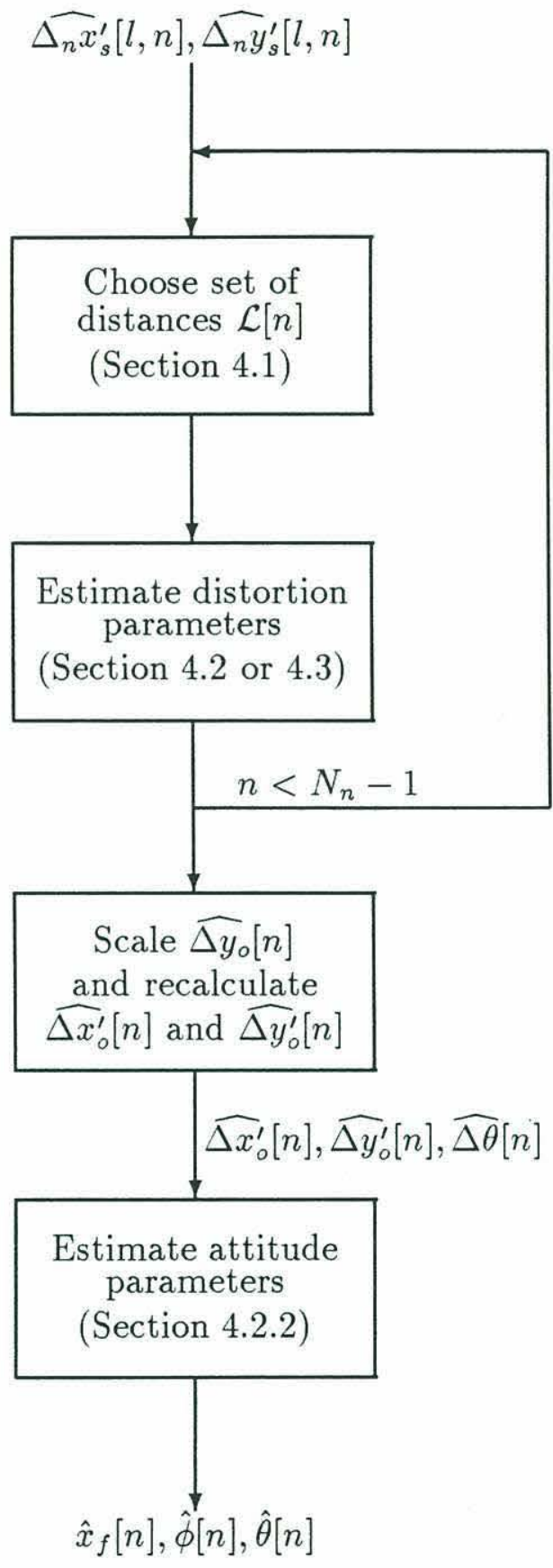

Figure 4.2: Technique for estimation of the distortion and attitude parameters. 


$$
\begin{aligned}
S_{l^{2}}[n] & \triangleq \sum_{l \in \mathcal{L}[n]} l^{2} \\
S_{x}[n] & \triangleq \sum_{l \in \mathcal{L}[n]} \widehat{\Delta_{n} x_{s}^{\prime}}[l, n] \\
S_{y}[n] & \triangleq \sum_{l \in \mathcal{L}[n]} \widehat{\Delta_{n} y_{s}^{\prime}}[l, n] \\
S_{l y}[n] & \triangleq \sum_{l \in \mathcal{L}[n]} l \widehat{\Delta_{n} y_{s}^{\prime}}[l, n] \\
\delta[n] & \triangleq p[n] S_{l^{2}}[n]-S_{l}^{2}[n],
\end{aligned}
$$

then the resulting estimates are given by

$$
\begin{aligned}
& \widehat{\Delta x_{o}^{\prime}}[n]=\frac{1}{p[n]} S_{x}[n] \\
& \widehat{\Delta y_{o}^{\prime}}[n]=\frac{S_{l^{2}}[n] S_{y}[n]-S_{l}[n] S_{l y}[n]}{\delta[n]} \\
& \widehat{\Delta \theta}[n]=\frac{p[n] S_{l y}[n]-S_{l}[n] S_{y}[n]}{\delta[n]} .
\end{aligned}
$$

It is now necessary to scale the estimates of $\Delta x_{o}^{\prime}[n]$ and $\Delta y_{o}^{\prime}[n]$ to compensate for the $\left(v / v_{\mathcal{N}}^{\prime}\right)$ factor introduced in Section 3.3.2. The first step is to obtain estimates of the yaw angle by cumulatively adding the increment estimates, $\widehat{\Delta \theta}[n]$. Thus,

$$
\hat{\theta}[n]=\hat{\theta}[0]+\sum_{i=0}^{n-1} \widehat{\Delta \theta}[n] .
$$

The estimate of the initial yaw angle, $\hat{\theta}[0]$ may be chosen, for instance, so that the average value of $\hat{\theta}[n]$ over the entire sonograph be zero. However, the choice of $\hat{\theta}[0]$ does not affect the correction of geometric distortions in the image, but only the orientation of the corrected image as a whole. If the assumption that the average yaw angle is zero is incorrect, the only error incurred is that the final image will be rotated from its true orientation by an angle equal to the difference between $\hat{\theta}[0]$ and the true initial yaw angle.

The next step is to obtain estimates of $x_{o}^{\prime}[n]$ and $y_{o}^{\prime}[n]$ in the $(x, y)$ coordinate system. We first convert the increments from the $\left(x^{\prime}, y^{\prime}\right)$ to the $(x, y)$ coordinate system, using 
Eqs. (2.13a) and (2.13b), obtaining

$$
\begin{aligned}
& \widehat{\Delta x}_{o}[n]=\widehat{\Delta x_{o}^{\prime}}[n] \cos \hat{\theta}[n]-\widehat{\Delta y_{o}^{\prime}}[n] \sin \hat{\theta}[n] \\
& \widehat{\Delta y}_{o}[n]=\widehat{\Delta y_{o}^{\prime}}[n] \cos \hat{\theta}[n]+\widehat{\Delta x_{o}^{\prime}}[n] \sin \hat{\theta}[n],
\end{aligned}
$$

The estimates of $\Delta y_{o}[n]$ must now be scaled to make their average value equal to $v T_{f}$, thus compensating for the $\left(v / v_{\mathcal{N}}^{\prime}\right)$ factor. In our case $v=3$ knots and $T_{f}=0.133 \mathrm{~s}$ (see Section 1.5), resulting in $v T_{f}=20 \mathrm{~cm}$. Before scaling, the average value of $\widehat{\Delta y}_{o}[n]$ is $22.2 \mathrm{~cm}$, meaning that $\left(v / v_{\mathcal{N}}^{\prime}\right)$ equals 1.11. Equations $(4.4 \mathrm{a})$ and $(4.4 \mathrm{~b})$ are now reversed so that $\widehat{\Delta x_{o}^{\prime}}[n]$ and $\widehat{\Delta y_{o}^{\prime}}[n]$ may be recalculated to compensate for the $\left(v / v_{\mathcal{N}}^{\prime}\right)$ factor. Thus,

$$
\begin{aligned}
& \widehat{\Delta x_{o}^{\prime}}[n]=\widehat{\Delta x}_{o}[n] \cos \hat{\theta}[n]+\left(v_{\mathcal{N}}^{\prime} / v\right){\widehat{\Delta y_{o}}}_{0}[n] \sin \hat{\theta}[n] \\
& \widehat{\Delta y_{o}^{\prime}}[n]=\left(v_{\mathcal{N}}^{\prime} / v\right){\widehat{\Delta y_{o}}}_{0}[n] \cos \hat{\theta}[n]-{\widehat{\Delta x_{o}}}_{0}[n] \sin \hat{\theta}[n]
\end{aligned}
$$

Figure 4.3 shows the estimates of distortion parameters obtained with this approach. Notice that the estimates of $\Delta x_{o}^{\prime}[n]$ and $\Delta y_{o}^{\prime}[n]$ have greater high-frequency content than the estimates of $\Delta \theta[n]$.

\subsubsection{Estimating the Attitude Parameters}

We now proceed to obtain estimates of the towfish attitude parameters from the estimates of distortion parameters. As indicated in Chapter 2, this is not required for correcting the geometric distortions in the image, but it is convenient to obtain estimates of parameters that describe more directly the position and orientation of the towfish.

The points $\left(x_{o}[n], y_{o}[n]\right)$ are estimated by cumulatively adding the estimates of the increments $\Delta x_{o}[n]$ and $\Delta y_{o}[n]$, in the latter case after multiplication by $\left(v_{\mathcal{N}}^{\prime} / v\right)$, i. e.,

$$
\begin{aligned}
& \hat{x}_{o}[n]=\hat{x}_{o}[0]+\sum_{i=0}^{n-1} \widehat{\Delta x}_{o}[i] \\
& \hat{y}_{o}[n]=\hat{y}_{o}[0]+\sum_{i=0}^{n-1}\left(v_{\mathcal{N}}^{\prime} / v\right) \widehat{\Delta y}_{0}[i],
\end{aligned}
$$

with the starting point arbitrarily chosen as the origin of the coordinate system,

$$
\hat{x}_{o}[0]=\hat{y}_{o}[0]=0 .
$$



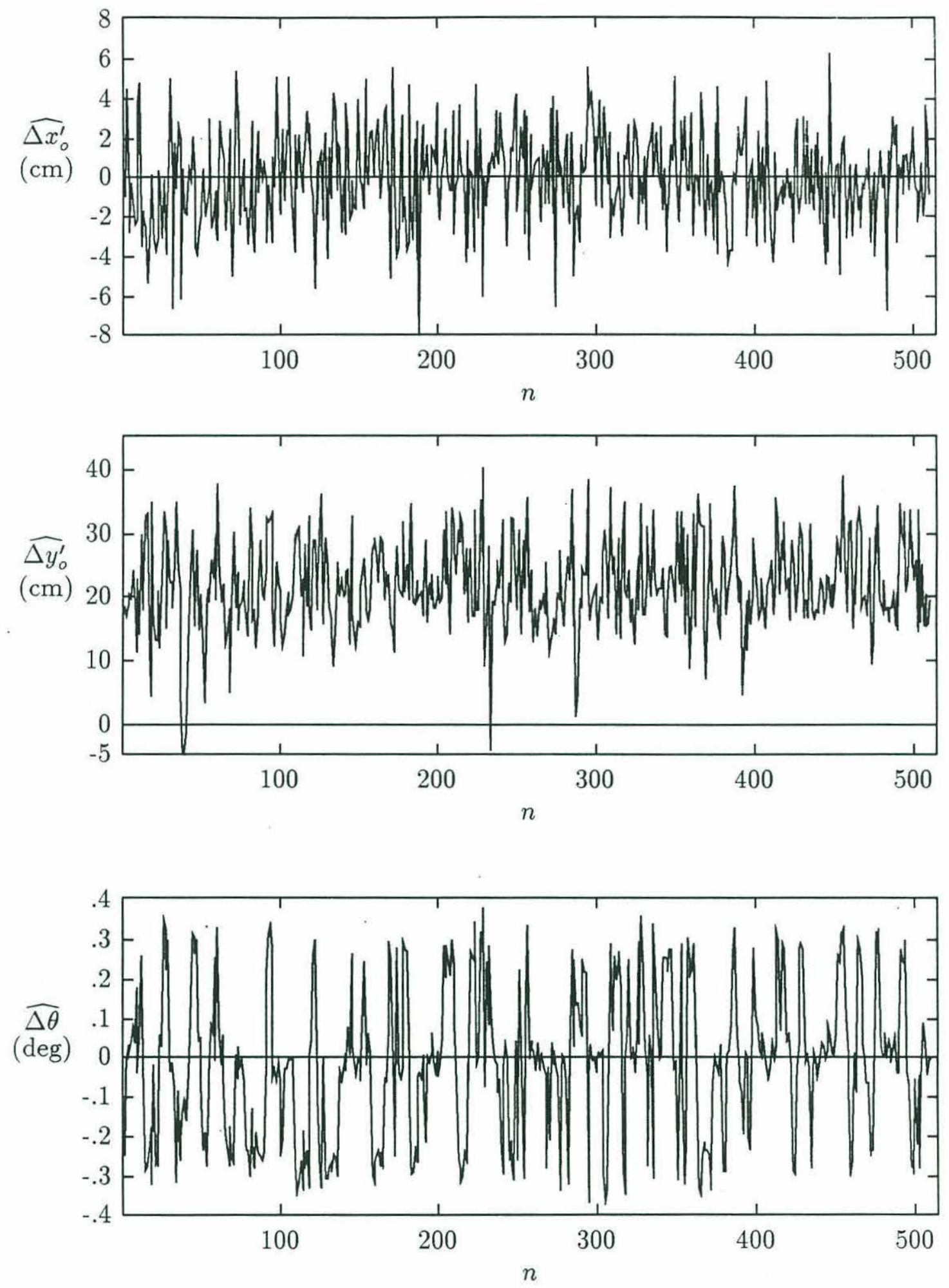

Figure 4.3: Estimates of distortion parameters obtained through deterministic leastsquares approximation. 
To estimate the attitude parameters, we use Eqs. (2.8a) and (2.8b), repeated here for convenience,

$$
\begin{aligned}
& x_{o}[n]=x_{f}[n]-z_{f}[n] \tan \phi[n] \sin \theta[n] \\
& y_{o}[n]=y_{f}[n]+z_{f}[n] \tan \phi[n] \cos \theta[n] .
\end{aligned}
$$

Referring to Fig. 2.4, we see that

$$
z_{f}[n]=h[n] \cos \phi[n]
$$

where $h[n]$ is the distance between the towfish and point $\left(x_{o}[n], y_{0}[n]\right)$ and can be measured directly from the sonograph as the height of the water column on each line. These three equations are all we have to obtain estimates of $x_{f}[n], y_{f}[n], z_{f}[n]$ and $\phi[n]$ from $h[n]$ and the estimates of $x_{o}[n], y_{\circ}[n]$ and $\theta[n]$. Therefore, it is possible to estimate only two of the attitude parameters, and the third one has to be estimated by different means. One solution to this problem is to assume that the along-track distance between the towfish and the deploying vessel remains constant during the acquisition of the sonograph. That allows $y_{f}[n]$ to be estimated from navigational measurements of the deploying vessel. If such measurements are not available, it is still possible to resort to the assumption that the towfish moved at a constant speed $v$, equal to the average speed of the deploying vessel. If $y_{f}[n]$ is measured, or assumed to satisfy these approximations, Eqs. (4.7a), (4.7b), and (4.8) may be solved for the remaining attitude parameters, lateral position, $x_{f}[n]$, and pitch angle, $\phi[n]$. Thus, with

$$
\hat{y}_{f}[n]=n v T_{f}
$$

we obtain from Eqs. (4.7b) and (4.8),

$$
\hat{\phi}[n]=\arcsin \left(\frac{\hat{y}_{o}[n]-n v T_{f}}{h[n] \cos \hat{\theta}[n]}\right) .
$$

Finally, from Eq. (4.7a),

$$
\hat{x}_{f}[n]=\hat{x}_{o}[n]+h[n] \sin \hat{\phi}[n] \sin \hat{\theta}[n]
$$

If measured values of $y_{f}[n]$ are not available, as in our case, and the approximation of (4.9) is adopted, then variations in towfish speed from the assumed fixed value $v$ affect 
the estimates of pitch angle, calculated through Eq. (4.10). Therefore, this equation should be used only with the clear understanding that the resulting estimates $\hat{\phi}[n]$ reflect not only the towfish pitch angle, but also variations in its speed.

Equations (4.3), (4.10), and (4.11) can be used for estimating $\theta[n], \phi[n]$, and $x_{f}[n]$ from $\widehat{\Delta x_{o}^{\prime}}[n], \widehat{\Delta y_{o}^{\prime}}[n]$ and $\widehat{\Delta \theta}[n]$. These equations can also be used for determining approximate confidence intervals for the resulting estimates. If the estimation errors, $v_{x}[l, n]$ and $v_{y}[l, n]$, are assumed to be Gaussian random variables, it can be shown [5] that the variances of the estimates of distortion parameters are given by

$$
\begin{aligned}
\sigma_{\overparen{\Delta x_{o}^{\prime}}}^{2}[n] & =\frac{1}{p[n]}\left(\frac{\varepsilon_{x}^{2}[n]}{p[n]-1}\right) \\
\sigma_{\overparen{\Delta y_{o}^{\prime}}}^{2}[n] & =\frac{S_{l^{2}}[n]}{\delta[n]}\left(\frac{\varepsilon_{y}^{2}[n]}{p[n]-2}\right) \\
\sigma_{\overparen{\Delta \theta}}^{2}[n] & =\frac{p[n]}{\delta[n]}\left(\frac{\varepsilon_{y}^{2}[n]}{p[n]-2}\right)
\end{aligned}
$$

Equations (4.4a) and (4.4b) may be used to obtain first-order estimates [43] for the variances of $\widehat{\Delta x}_{o}[n]$ and $\widehat{\Delta y}_{o}[n]$,

$$
\begin{aligned}
\sigma_{\widehat{\Delta x_{o}}}^{2}[n]= & \left(\frac{\partial \widehat{\Delta x_{o}}}{\partial \widehat{\Delta x_{o}^{\prime}}}\right)^{2} \sigma_{\widehat{\Delta x_{o}^{\prime}}}^{2}[n]+\left(\frac{\partial \widehat{\Delta x_{o}}}{\partial \widehat{\Delta y_{o}^{\prime}}}\right)^{2} \sigma_{\widehat{\Delta y_{o}^{\prime}}}^{2}[n]+\left(\frac{\partial \widehat{\Delta x_{o}}}{\partial \hat{\theta}}\right)^{2} \sigma_{\hat{\theta}}^{2}[n] \\
= & \cos ^{2} \hat{\theta}[n] \sigma_{\widehat{\Delta x_{o}^{\prime}}}^{2}[n]+\sin ^{2} \hat{\theta}[n] \sigma_{\widehat{\Delta y_{o}^{\prime}}}^{2}[n] \\
& +\left(\widehat{\Delta x_{o}^{\prime}}[n] \sin \hat{\theta}[n]+\widehat{\Delta y_{o}^{\prime}}[n] \cos \hat{\theta}[n]\right)^{2} \sigma_{\hat{\theta}}^{2}[n]
\end{aligned}
$$

and

$$
\begin{aligned}
\sigma_{\widehat{\Delta y_{o}}}^{2}[n]= & \left(\frac{\partial \widehat{\Delta y_{o}}}{\partial \widehat{\Delta x_{o}^{\prime}}}\right)^{2} \sigma_{\widehat{\Delta x_{o}^{\prime}}}^{2}[n]+\left(\frac{\partial \widehat{\Delta y_{o}}}{\partial \widehat{\Delta y_{o}^{\prime}}}\right)^{2} \sigma_{\widehat{\Delta y_{o}^{\prime}}}^{2}[n]+\left(\frac{\partial \widehat{\Delta y_{o}}}{\partial \hat{\theta}}\right)^{2} \sigma_{\hat{\theta}}^{2}[n] \\
= & \cos ^{2} \hat{\theta}[n] \sigma_{\widehat{\Delta y_{o}^{\prime}}}^{2}[n]+\sin ^{2} \hat{\theta}[n] \sigma_{\widehat{\Delta x_{o}^{\prime}}}^{2}[n] \\
& +\left(\widehat{\Delta x_{o}^{\prime}}[n] \cos \hat{\theta}[n]-\widehat{\Delta y_{o}^{\prime}}[n] \sin \hat{\theta}[n]\right)^{2} \sigma_{\hat{\theta}}^{2}[n] .
\end{aligned}
$$

Making the simplifying assumption that the increment estimates $\widehat{\Delta \theta}[n], \widehat{\Delta x_{0}}[n]$, and $\widehat{\Delta y}_{0}[n]$ for different lines of the sonograph are independent, we have

$$
\sigma_{\hat{\theta}}^{2}[n]=\sum_{i=0}^{n} \sigma_{\widehat{\Delta \theta}}^{2}[i]
$$




$$
\begin{aligned}
& \sigma_{\hat{x}_{o}}^{2}[n]=\sum_{i=0}^{n} \sigma_{\widehat{\Delta x_{o}}}^{2}[i] \\
& \sigma_{\hat{y}_{o}}^{2}[n]=\sum_{i=0}^{n} \sigma_{\widehat{\Delta y_{o}}}^{2}[i] .
\end{aligned}
$$

By adding $\pm 2 \sigma_{\hat{x}_{o}}[n], \pm 2 \sigma_{\hat{y}_{o}}[n]$, and $\pm 2 \sigma_{\hat{\theta}}[n]$ to $\hat{x}_{o}[n], \hat{y}_{o}[n]$, and $\hat{\theta}[n]$, respectively, we obtain confidence intervals for these estimates. If the assumptions made above are valid, namely, that the estimation errors are Gaussian random variables, that the increment estimates for different lines are independent, and that the first-order estimates of $\sigma_{\triangle x_{o}}^{2}[n]$ and $\sigma_{\Delta_{o}}^{2}[n]$ are accurate, then these confidence intervals define the ranges within which $x_{o}[n], y_{o}[n]$, and $\theta[n]$ can be expected to be, with $95 \%$ probability.

By applying Eqs. (4.11) and (4.10) to the lower and upper limits of the confidence intervals of $\hat{x}_{o}[n]$ and $\hat{y}_{o}[n]$ we also obtain confidence intervals for $\hat{x}_{f}[n]$ and $\hat{\phi}[n]$. The resulting estimates of attitude parameters and their confidence intervals are shown in Fig. 4.4. In view of all the approximations and simplifying assumptions that were made in the derivation of the confidence intervals, they should be considered only as approximate indicators of the magnitude of the estimation errors of the attitude parameters. For that reason, they are not labeled as $95 \%$ confidence intervals in the figures. However, notice that, though the precise significance of these confidence intervals is not rigorously established, they are useful for indicating relative trends in the evolution of the estimates and in their relative accuracy with respect to one another. For instance, notice that the confidence intervals grow larger with increasing $n$, since calculating the attitude parameters involves cumulatively adding the estimates of increments of distortion parameters, $\widehat{\Delta x_{o}^{\prime}}[n], \widehat{\Delta y_{o}^{\prime}}[n]$, and $\widehat{\Delta \theta}[n]$. Thus, the estimation error adds up and increases with $n$. The confidence intervals also indicate that the estimates of yaw angle, $\hat{\theta}[n]$, are more accurate than the estimates of pitch angle, $\hat{\phi}[n]$. That is explained by the fact that during the acquisition of our sonographs, the altitude of the towfish above the bottom was approximately one tenth of the maximum range (as is commonly the case). Thus, variations in yaw angle cause a displacement of the scan lines at long ranges that is 10 times larger than the displacement caused by equal variations in pitch angle. At shorter ranges the difference is not so marked, but still the average effect of yawing over all ranges is greater than the average effect of pitching, resulting in better estimates of the yaw angle. 

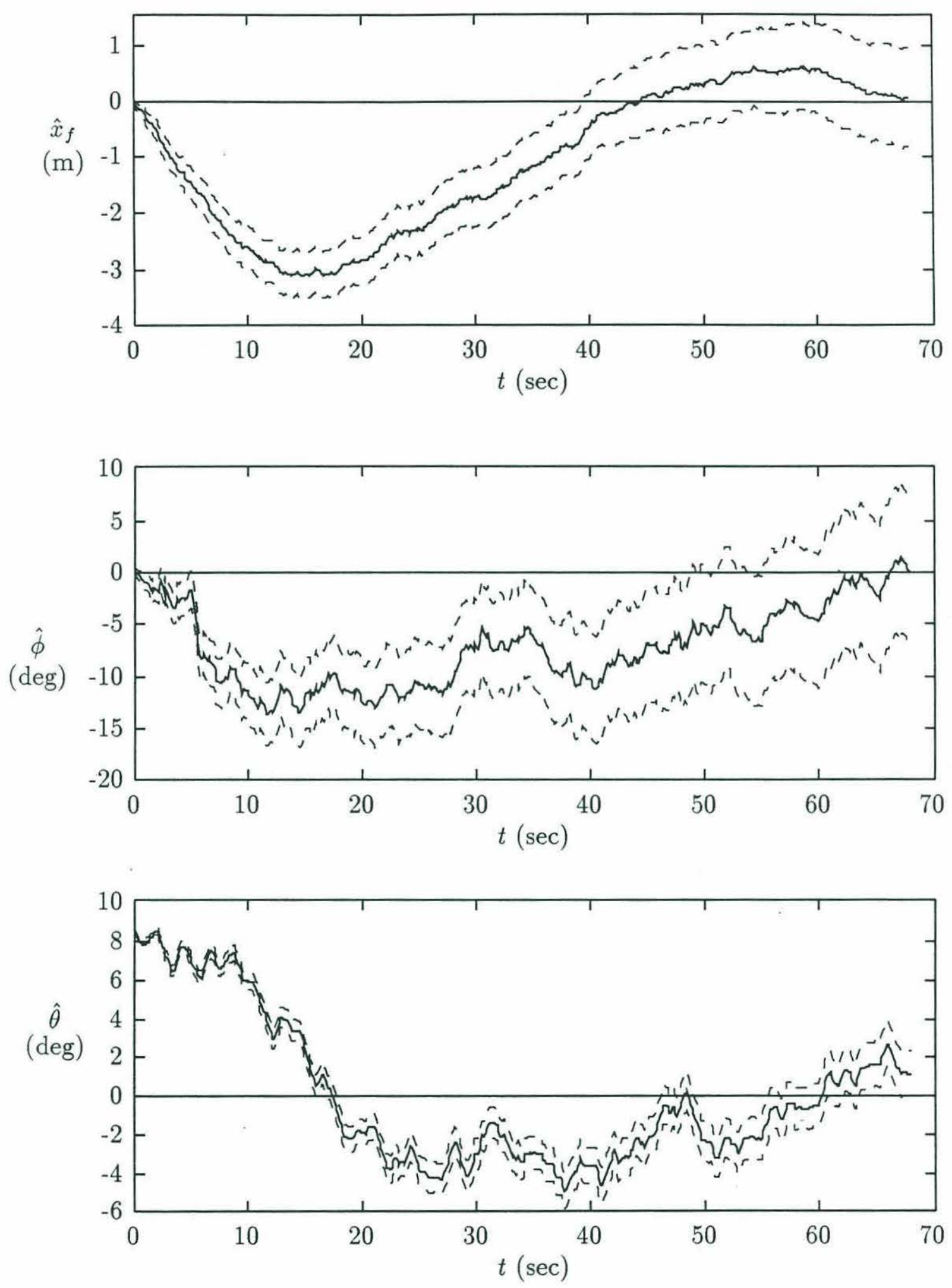

Figure 4.4: Estimates of attitude parameters obtained through deterministic least-squares estimation. The confidence intervals are indicated by dashed lines. 


\subsection{Bayesian Linear Least-Squares Estimation}

Another approach to the estimation of the distortion parameters is to use Bayesian least-squares (or minimum variance) estimation, which incorporates statistical information about the parameters and measurement noise, to minimize the variance of the estimation error [43]. Ideally, the statistical properties of the parameter and noise vectors should be derived independently, from experiments or from a theoretical analysis of the dynamics of the towfish and of the autocorrelation of the backscattering function of the bottom, respectively. It is also possible to attempt to derive such information from the same data from which the distortion parameters are estimated, but caution should be exercised in the interpretation of the results thus obtained. This section presents procedures for Bayesian linear least-squares estimation of the distortion parameters, first from each line separately, and then by recursively updating the estimates at each line. These procedures are described with the intent of providing a framework in which independently derived statistical information about the parameters and measurement noise may be easily incorporated in the future.

\subsubsection{Line-by-Line Estimation}

The correlation between the distortion parameters and measurement noise can be described more compactly in matrix form if we write Eqs. (4.1a) and (4.1b) as

$$
\underline{\zeta}[n]=C[n] \underline{\xi}[n]+\underline{v}[n],
$$

where $\zeta[n], \underline{\xi}[n]$ and $\underline{v}[n]$ are the vectors

$$
\underline{\zeta}[n] \triangleq\left[\begin{array}{c}
\widehat{\Delta_{n} x_{s}^{\prime}}\left[l_{1}, n\right] \\
\vdots \\
\widehat{\Delta_{n} x_{s}^{\prime}}\left[l_{p}, n\right] \\
\widehat{\Delta_{n} y_{s}^{\prime}}\left[l_{1}, n\right] \\
\vdots \\
\widehat{\Delta_{n} y_{s}^{\prime}}\left[l_{p}, n\right]
\end{array}\right] \quad \underline{\xi}[n] \triangleq\left[\begin{array}{c}
\Delta x_{o}^{\prime}[n] \\
\Delta y_{o}^{\prime}[n] \\
\Delta \theta[n]
\end{array}\right] \quad \underline{v}[n] \triangleq\left[\begin{array}{c}
v_{x}\left[l_{1}, n\right] \\
\vdots \\
v_{x}\left[l_{p}, n\right] \\
v_{y}\left[l_{1}, n\right] \\
\vdots \\
v_{y}\left[l_{p}, n\right]
\end{array}\right],
$$


and $C[n]$ is the observation matrix

$$
C[n] \triangleq\left[\begin{array}{ccc}
1 & 0 & 0 \\
\vdots & \vdots & \vdots \\
1 & 0 & 0 \\
0 & 1 & l_{1}\left(c T_{s} / 2\right) \\
\vdots & \vdots & \\
0 & 1 & l_{p}\left(c T_{s} / 2\right)
\end{array}\right] .
$$

Let $m_{\zeta}[n]$ and $m_{\xi}[n]$ denote the expected values of $\underline{\zeta}[n]$ and $\underline{\xi}[n]$, respectively, and assume that the expected value of $\underline{v}[n]$ is zero. Let $\Lambda_{\zeta}[n], \Lambda_{\xi}[n]$ and $R[n]$ denote their covariance matrices. Further, let $\Lambda_{\xi \zeta}[n]$ and $\Lambda_{\xi v}[n]$ denote the cross-covariance matrices of $\underline{\xi}[n]$ with $\underline{\zeta}[n]$ and $\underline{v}[n]$, respectively. ${ }^{1}$ Then the linear minimum variance estimate of $\underline{\xi}[n]$ given $\underline{\zeta}[n]$ is given by $[43]$

$$
\hat{\xi}[n]=m_{\xi}[n]+\Lambda_{\xi \zeta}[n] \Lambda_{\zeta}^{-1}[n]\left(\underline{\zeta}[n]-m_{\zeta}[n]\right),
$$

where, from Eq. (4.11),

$$
\begin{aligned}
m_{\zeta}[n] & =C[n] m_{\xi}[n] \\
\Lambda_{\xi \zeta}[n] & =\Lambda_{\xi}[n] C^{T}[n]+\Lambda_{\xi v}[n] \\
\Lambda_{\zeta}[n] & =C[n] \Lambda_{\xi}[n] C^{T}[n]+C[n] \Lambda_{\xi v}[n]+\Lambda_{\xi v}^{T}[n] C^{T}[n]+R[n] .
\end{aligned}
$$

If numerical values for the covariance matrices are stipulated theoretically or measured from the data itself, these equations may then be used for estimating the distortion parameter vector $\underline{\xi}[n]$ on a line-by-line basis.

\subsubsection{Recursive Estimation}

Bayesian least-squares estimation seeks to minimize the variance of the estimation error by exploiting the correlation between the components of the parameter and noise vectors at time $n$. However, it is also possible to take into account their temporal correlation. In other words, the distortion parameter vector $\xi[n]$ can be estimated through

\footnotetext{
${ }^{1}$ In this problem, $\Lambda_{\xi v}[n]$ is in general non-zero because the amplitude of the measurement noise can be affected by the degree of geometric distortion, and is therefore correlated with the distortion parameters.
} 
stochastic filtering. In this particular problem, a Kalman filter is a natural choice, since Kalman filters assume that the temporal correlation of the parameters to be estimated can be described by a state-space model, which in our case is a reasonable assumption, given that our parameters are related to the evolution of a hydrodynamic system. We employ a standard Kalman filter to obtain a causal estimation procedure, though it is also possible to use smoothing.

For an $N$ th-order state space model, the 3 -dimensional vectors of distortion parameters will be stacked to form a $3 N$-dimensional state vector

$$
\Xi[n] \triangleq\left[\begin{array}{c}
\underline{\xi}[n] \\
\underline{\xi}[n-1] \\
\vdots \\
\underline{\xi}[n-(N-1)] .
\end{array}\right]
$$

Adding to our model an $N$ th-order state propagation equation, we obtain the state-space model

$$
\begin{aligned}
\underline{\Xi}[n+1] & =A[n] \underline{\Xi}[n]+B[n] \underline{w}[n] \\
\hat{\zeta}[n] & =\bar{C}[n] \underline{\Xi}[n]+\underline{v}[n],
\end{aligned}
$$

where $\underline{w}[n]$ is a white process noise vector. For the second equation to be equivalent to Eq. (4.11), the new observation matrix is given by

$$
\bar{C}[n] \triangleq[C[n] \quad 0 \quad \cdots \quad 0] .
$$

Following the notation commonly adopted in the literature, the covariance matrices of the noise vectors $\underline{w}[n]$ and $\underline{v}[n]$ will be denoted by $Q[n]$ and $R[n]$, respectively, and their cross-covariance matrix will be denoted by $S[n]$. Two issues will be addressed next: how to use this state-space model to recursively estimate the parameters, and how to determine the unknown matrices $A[n], B[n], Q[n], R[n]$, and $S[n]$ of the model.

One way to determine the matrices of the state-space model of Eq. (4.15) is through a theoretical analysis of the towfish dynamics, possibly in conjunction with the estimation of some of the parameters in the theoretical model from actual attitude measurements obtained through experiments in which sensors are mounted on the towfish. If the model 
thus derived still contains unknown parameters or time-varying parameters that depend on the conditions during the survey and cannot be determined beforehand through experiments, then these parameters and the state vector itself may be concurrently estimated through adaptive Kalman filtering.

The general problem we face is to simultaneously estimate the state vectors $\Xi[n]$ and a number of unknown parameters of the state-space model, which will be combined into a parameter vector $\underline{\alpha}[n]$. In the next sections we consider how the state vectors and model parameters can be estimated separately by two recursive algorithms and how these algorithms can be combined to form an adaptive Kalman filter. Our treatment of these algorithms is not exhaustive; the intent here is to provide a general framework for the application of recursive estimation and identification techniques to the results derived in Chapters 2 and 3 .

\section{Recursive Identification of the State Vector}

The discrete-time Kalman filter is the optimal solution to the problem of recursively calculating the Bayesian linear least-squares estimate of the present value of a discretetime stochastic process given the present and past values of another stochastic process. It assumes that the dynamics of the process to be estimated and its relationship with the observation process can be described through a state-space model. The details of the derivation of the Kalman filter equations can be found in [1] and [26]. Using a standard Kalman filter would require inverting a matrix of order $2 p$, the dimension of the observation vector $\zeta[n]$, in the measurement update step of each iteration. In our case, it is more computationally efficient to use the so-called information filter version of the Kalman filter, that requires the inversion of a matrix of order $3 N$, the dimension of the state vector $\Xi[n]$. The information filter uses the quantities

$$
\begin{aligned}
\underline{\hat{a}}[n \mid n-1] & \triangleq \beta^{2 n} P^{-1}[n \mid k-1] \hat{\Xi}[n \mid n-1] \\
\underline{\hat{a}}[n \mid n] & \triangleq \beta^{2 n} P^{-1}[n \mid n] \hat{\Xi}[n \mid n],
\end{aligned}
$$


where $P[n \mid n]$ and $P[n \mid n-1]$ are the variances of the estimation and prediction errors, respectively, i. e.,

$$
\begin{gathered}
P[n \mid n] \triangleq E\left\{(\hat{\Xi}[n \mid n]-\Xi[n])(\hat{\Xi}[n \mid n]-\Xi[n])^{T}\right\} \\
P[n \mid n-1] \triangleq E\left\{(\hat{\Xi}[n \mid n-1]-\Xi[n])(\hat{\Xi}[n \mid n-1]-\Xi[n])^{T}\right\},
\end{gathered}
$$

and $\beta$ is a forgetting factor, $0<\beta \leq 1$, used to decrease the weight of past observations. With $\beta=1$ we obtain the standard Kalman filter. In the case of a linear model, such as the one given in (4.15), the estimates of the state vector that minimize $P[n \mid n]$ can be calculated recursively according to the following equations:

Measurement Update Step

$$
\begin{aligned}
\underline{\hat{a}}[n \mid n] & =\underline{\hat{a}}[n \mid n-1]+\bar{C}^{T}[n] R^{-1}[n] \underline{\zeta}[n] \\
P^{-1}[n \mid n] & =P^{-1}[n \mid n-1]+\beta^{-2 n} \bar{C}^{T}[n] R^{-1}[n-1] \bar{C}[n] \\
\hat{\Xi}[n \mid n] & =\beta^{-2 n} P[n \mid n] \underline{\hat{a}}[n \mid n]
\end{aligned}
$$

Prediction Step

$$
\begin{aligned}
F[n] & =A[n]-B[n] S[n] R^{-1}[n] \bar{C}[n] \\
G[n] & =\beta^{2(n+1)} F^{-T}[n] P^{-1}[n \mid n] F^{-1}[n] \\
H[n] & =G[n] B[n]\left(B^{T}[n] G[n] B[n]+\left(Q[n]-S[n] R^{-1}[n] S[n]\right)^{-1}\right)^{-1} \\
\underline{\hat{a}}[n+1 \mid n] & =\left(I-H[n] B^{T}[n]\right)\left(F^{-T}[n] \underline{\hat{a}}[n \mid n]+G[n] B[n] S[n] R^{-1}[n] \underline{\zeta}[n]\right) \\
P^{-1}[n+1 \mid n] & =\beta^{-2 n}\left(I-H[n] B^{T}[n]\right) G[n]
\end{aligned}
$$

These equations assume that the process noise $\underline{w}[n]$ and the measurement noise $\underline{v}[n]$ are white. If that is not the case, the state-space model can be augmented to incorporate a model of the temporal autocorrelation of the noise processes.

\section{Recursive Least-Squares Identification of the Model Parameters}

An autoregressive (AR) model was adopted for the dynamics of the state vector. While this is not the most general model that could be adopted, it illustrates this technique without unnecessarily complicating its formulation. In fact, an autoregressive 
model can be easily incorporated into the state-space model of (4.15) by simply adopting a companion-form matrix $A[n]$. The $N$ th-order autoregressive model given by

$$
\underline{\xi}[n+1]=A_{1} \underline{\xi}[n]+A_{2} \underline{\xi}[n-1]+\ldots+A_{N} \underline{\xi}[n-N+1]+\underline{w}[n],
$$

corresponds to Eq. (4.15) with

$$
A[n]=\left[\begin{array}{ccccc}
A_{1}[n] & A_{2}[n] & \cdots & A_{N-1}[n] & A_{N}[n] \\
I & 0 & 0 & \cdots & 0 \\
0 & I & 0 & \cdots & 0 \\
\vdots & \ddots & \ddots & \ddots & \vdots \\
0 & \cdots & 0 & I & 0
\end{array}\right] \text { and } B[n]=\left[\begin{array}{c}
I \\
0 \\
\vdots \\
0
\end{array}\right] \text {, }
$$

where $N$ is the dimension of the parameter vector and where $I$ and 0 represent identity and zero matrices of dimension equal to the number of parameters used in the state space model, in our case, three (namely, $\Delta x_{o}^{\prime}[n], \Delta y_{o}^{\prime}[n]$ and $\Delta \theta[n]$ ).

Our objective is to estimate the blocks $A_{1}[n], A_{2}[n], \ldots, A_{N}[n]$ of matrix $A[n]$. It will be useful to combine the first three rows of $A[n]$, which contain these sub-matrices, into a $9 N$-dimensional parameter vector

$$
\underline{\alpha}[n] \triangleq\left[\begin{array}{c}
(A)_{1}^{T}[n] \\
(A)_{2}^{T}[n] \\
(A)_{3}^{T}[n]
\end{array}\right],
$$

where $(A)_{i}[n]$ represents the $i$ th row of $A[n]$. It will also be useful to rearrange the state vectors into a new $(3 \times 9 N)$-dimensional matrix

$$
\chi[n] \triangleq\left[\begin{array}{ccc}
\Xi^{T}[n] & 0 & 0 \\
0 & \Xi^{T}[n] & 0 \\
0 & 0 & \Xi^{T}[n]
\end{array}\right],
$$

which is the multidimensional counterpart of what is usually called the regression vector in the one-dimensional case. The autoregressive model may be used to predict the next value of the state vector from the present and $(N-1)$ past values. Denoting by $\hat{\xi}[n+1 \mid n]$ the predicted value of $\xi[n+1]$ given $\xi[n], \xi[n-1], \ldots, \xi[n-N+1]$, we have

$$
\hat{\xi}[n+1 \mid n]=\chi[n] \underline{\alpha}[n] .
$$


There are several possible criteria for estimating the model parameters $\underline{\alpha}[n]$ from the vectors $\underline{\xi}[n][38]$. One is to minimize the mean-square value of the prediction error. This criterion motivates a number of closely related algorithms, among which is found the weighted least-squares method. In this method the parameter estimate is chosen according to

$$
\underline{\hat{\alpha}}[n]=\underset{\underline{\alpha}}{\arg \min } \sum_{k=0}^{n} \beta^{2(n-k)}(\underline{\xi}[k+1]-\underline{\hat{\xi}}[k+1 \mid k])^{T} Q^{-1}[k](\underline{\xi}[k+1]-\underline{\hat{\xi}}[k+1 \mid k]),
$$

where $\beta$ is again a forgetting factor satisfying $0<\beta \leq 1$. The recursive version of the weighted least-squares algorithm consists of calculating a new estimate $\underline{\alpha}[n]$ once $\underline{\xi}[n]$ is available, and then using it to calculate the prediction $\underline{\hat{\xi}}[n+1 \mid n]$ of its next value. It can be shown that if these estimates are to satisfy the least-squares criterion given above, they can be computed recursively as

$$
\begin{aligned}
J[n]= & U[n-1] \chi^{T}[n]\left(\beta^{2} Q[n]+\chi[n] U[n-1] \chi^{T}[n]\right)^{-1} \\
\underline{\hat{\alpha}}[n]= & \underline{\hat{\alpha}}[n-1]+J[n](\underline{\xi}[n]-\chi[n] \underline{\hat{\alpha}}[n-1]) \\
U[n]= & \frac{1}{\beta^{2}}\left(U[n-1]-U[n-1] \chi^{T}\left(\beta^{2} Q[n]+\chi[n] U[n-1] \chi^{T}[n]\right)^{-1}\right. \\
& \chi[n] U[n-1]) .
\end{aligned}
$$

For the derivation of the recursive least-squares algorithm see [38].

\section{The Extended Least-Squares Method}

If the model parameters are known, then $\hat{\underline{\Xi}}[n]$ can be calculated from the observations $\zeta[n]$ through a Kalman filter. Conversely, if the state vectors are known, then $\underline{\hat{\alpha}}[n]$ can be calculated from them and the observations through the recursive least-squares method. Figure 4.5(a) depicts this situation. When both the state and parameter vectors are unknown, the Kalman filter and the recursive least-squares algorithm can be combined into what is known as the extended least-squares method, as shown Fig. 4.5(b). It consists of calculating the parameter estimate $\underline{\hat{\alpha}}[n]$ at each iteration from the present and past state vector estimates, and then using it in the prediction step of the Kalman filter. The state and parameter estimates obtained through this method are suboptimal 

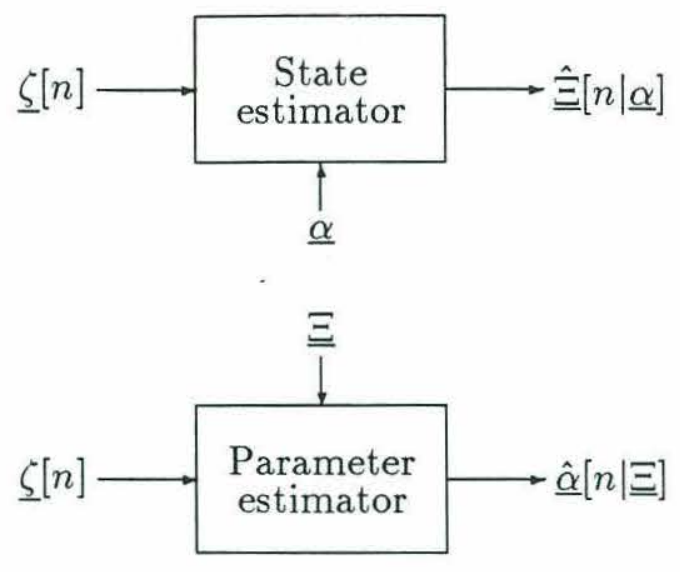

(a)

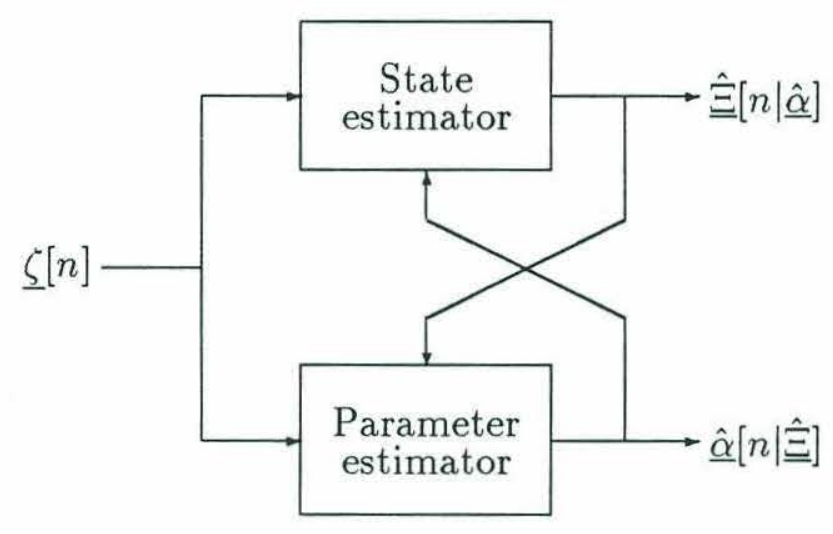

(b)

Figure 4.5: The Kalman filter and recursive least-squares algorithms (a) are combined in the extended least-squares method (b).

because they are not jointly estimated, but they can be shown to converge to the optimal estimates under certain conditions [1].

Since the covariance matrices are unknown, they are estimated by recursively computing the average of the outer products of the residual errors from the state and model parameter estimation steps. This too is a suboptimal procedure that, under certain circumstances, can be shown to converge to the optimal estimates [40]. The complete set of equations for the extended least-squares algorithm is: 


\section{State Estimation}

$$
\begin{aligned}
\underline{\hat{a}}[n \mid n] & =\underline{\hat{a}}[n \mid n-1]+\bar{C}^{T}[n] \widehat{R^{-1}}[n-1] \underline{\zeta}[n] \\
P^{-1}[n \mid n] & =P^{-1}[n \mid n-1]+\beta^{-2 n} \bar{C}^{T}[n] \widehat{R^{-1}}[n] \bar{C}[n] \\
\hat{\Xi}[n \mid n] & =\beta^{-2 n} P[n \mid n] \underline{\hat{a}}[n \mid n]
\end{aligned}
$$

\section{Model Estimation}

$$
\begin{aligned}
J[n]= & U[n-1] \hat{\chi}^{T}[n \mid n]\left(\beta^{2} \hat{Q}[n-1]+\chi[n \mid n] U[n-1] \hat{\chi}^{T}[n \mid n]\right)^{-1} \\
\underline{\hat{\alpha}}[n]= & \underline{\hat{\alpha}}[n-1]+J[n](\hat{\xi}[n \mid n]-\hat{\chi}[n \mid n] \underline{\hat{\alpha}}[n-1]) \\
U[n]= & \frac{1}{\beta^{2}}\left(U[n-1]-U[n-1] \hat{\chi}^{T}[n \mid n]\left(\beta^{2} \hat{Q}[n]+\hat{\chi}[n \mid n] U[n-1] \hat{\chi}^{T}[n \mid n]\right)^{-1}\right. \\
& \hat{\chi}[n \mid n] U[n-1]) .
\end{aligned}
$$

\section{Estimation of Covariance Matrices}

$$
\begin{aligned}
& \underline{\hat{\hat{w}}}[n]=\underline{\hat{\xi}}[n \mid n]-\hat{\chi}[n-1 \mid n-1] \hat{\alpha}[n] \\
& \underline{\hat{v}}[n]=\underline{\zeta}[n]-\bar{C}[n] \underline{\Xi}[n \mid n] \\
& \hat{Q}[n]=\frac{1-\beta^{2}}{1-\beta^{2(n+2)}}\left(\left(\frac{1-\beta^{2(n+1)}}{1-\beta^{2}}\right) \beta^{2} \hat{Q}[n-1]+\underline{\hat{w}}[n] \underline{\hat{w}}^{T}[n]\right) \\
& \widehat{R^{-1}}[n]=\frac{1-\beta^{2(n+2)}}{\beta^{2}\left(1-\beta^{2(n+1)}\right)}\left(R^{-1}[n-1]-\frac{\widehat{R^{-1}}[n-1] \underline{\hat{v}}[n]\left(\widehat{R^{-1}}[n-1] \underline{\hat{v}}[n]\right)^{T}}{\underline{v}^{T}[n] \widehat{R^{-1}}[n-1] \underline{\hat{v}}[n]+\frac{\beta^{2}\left(1-\beta^{2(n+1)}\right)}{1-\beta^{2}}}\right) \\
& \hat{S}[n]=\frac{1-\beta^{2}}{1-\beta^{2(n+2)}}\left(\left(\frac{1-\beta^{2(n+1)}}{1-\beta^{2}}\right) \beta^{2} \hat{S}[n-1]+\underline{\hat{v}}[n] \underline{\hat{w}}^{T}[n]\right)
\end{aligned}
$$

\section{State Prediction}

$$
\begin{aligned}
F[n] & =\hat{A}[n]-B[n] \hat{S}[n] \widehat{R^{-1}}[n] \bar{C}[n] \\
G[n] & =\beta^{2(n+1)} F^{-T}[n] P^{-1}[n \mid n] F^{-1}[n] \\
H[n] & =G[n] B[n]\left(B^{T}[n] G[n] B[n]+\left(\hat{Q}[n]-\hat{S}[n] \widehat{R^{-1}}[n] \hat{S}[n]\right)^{-1}\right)^{-1} \\
\underline{\hat{a}}[n+1 \mid n] & =\left(I-H[n] B^{T}[n]\right)\left(F^{-T}[n] \underline{\hat{a}}[n \mid n]+G[n] B[n] \hat{S}[n] \widehat{R^{-1}}[n] \zeta[n]\right) \\
P^{-1}[n+1 \mid n] & =\beta^{-2 n}\left(I-H[n] B^{T}[n]\right) G[n]
\end{aligned}
$$

Figure 4.6 shows the estimates of distortion parameters obtained through this algo- 
rithm, with $N=4$ and $\beta=1$. The initial state estimate was

$$
\hat{\xi}[0 \mid-1]=\left[\begin{array}{c}
0 \\
v T_{f} \\
0
\end{array}\right],
$$

and initial estimates for the covariance matrices were obtained from the parameter and error estimates of the deterministic least-squares algorithm. The initial estimates of the state-space model parameters corresponding to the autoregressive coefficients of $\Delta x_{o}^{\prime}[n], \Delta y_{o}^{\prime}[n]$, and $\Delta \theta[n]$ were equal to $1 / N$ and all the coefficients for the cross terms were equal to zero. Thus, recalling the definition of $\alpha[n]$ in Eq. (4.19), we have, for $N=4$,

$$
\hat{\alpha}_{i}[-1]= \begin{cases}0.25, & \text { for } i=1,4,7,10,14,17,20,23,27,30,33,36, \\ 0, & \text { otherwise. }\end{cases}
$$

With this choice of the $\hat{\alpha}_{i}[n]$ the initial autoregressive model amounts to taking the prediction of the next value of the state vector as the average of its last $N$ values. Finally, the initial covariance matrix $U[-1]$ was a $9 N$-dimensional identity matrix.

The state-space model causes the estimates of $\Delta x_{o}^{\prime}[n]$ and $\Delta y_{o}^{\prime}[n]$ to have less highfrequency content than the corresponding estimates obtained through the deterministic least-squares algorithm. The estimates of $\Delta \theta[n]$, however, do not change as much since they already have low high-frequency content in the deterministic least-squares case, probably as a result of the smaller estimation errors achieved for that parameter. Figure 4.7 shows the estimates of attitude parameters for the extended least-squares algorithm. These do not differ significantly from the estimates obtained through deterministic least-squares estimation, which could be expected since the only a priori information introduced was the autoregressive structure of the state-space model, and its order. If some of the model parameters are determined through a theoretical analysis of the towfish dynamics or through experiments, then a greater difference may result between the estimates obtained through the two algorithms. Notice, however, that the confidence intervals resulting from the extended least-squares algorithm are tighter than those obtained through deterministic least-squares estimation. This is a consequence of the reduction of the variance of the estimation error that is theoretically expected 

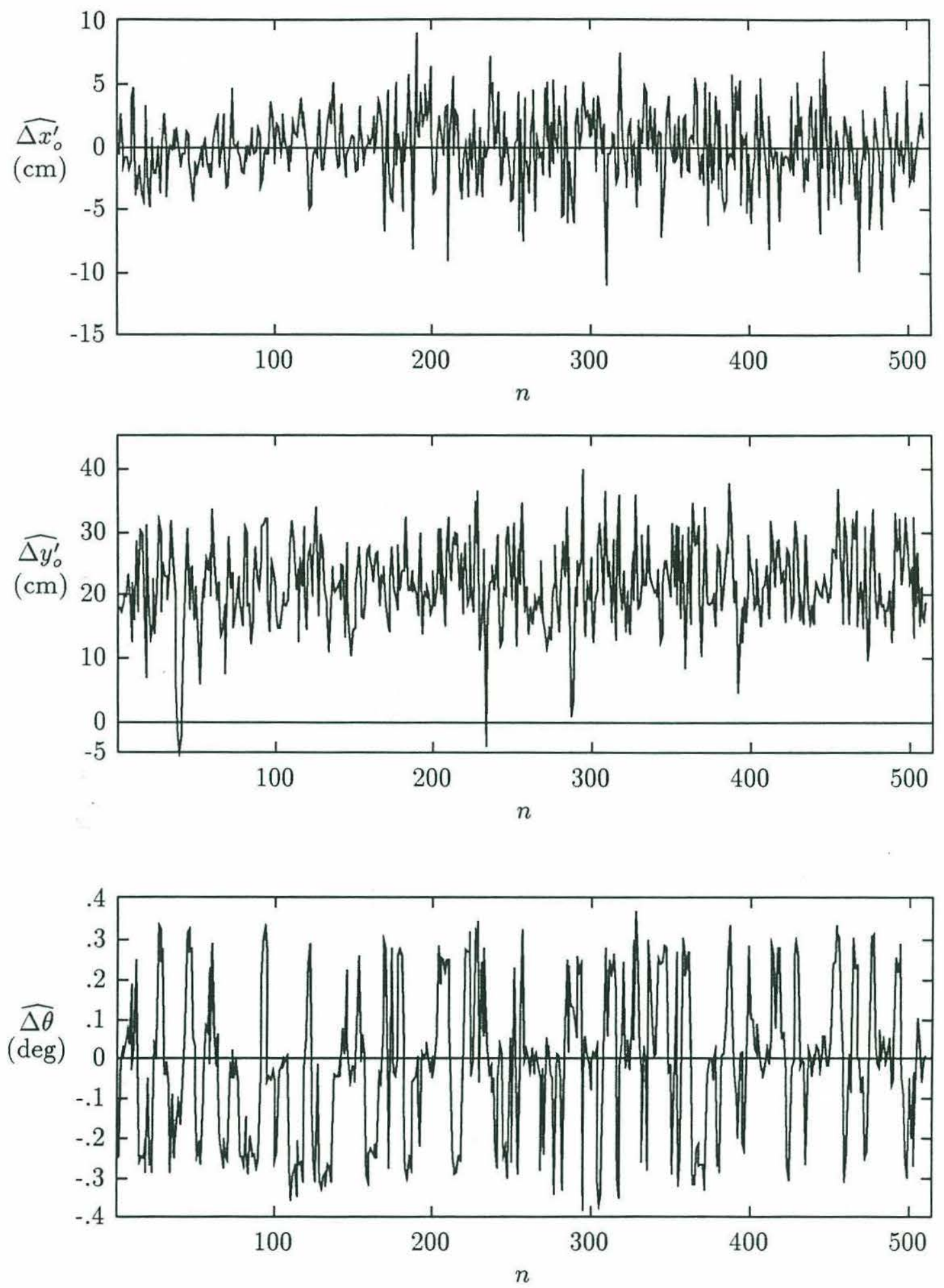

Figure 4.6: Estimates of distortion parameters obtained through the extended leastsquares algorithm with $N=4$ and $\beta=1$. 

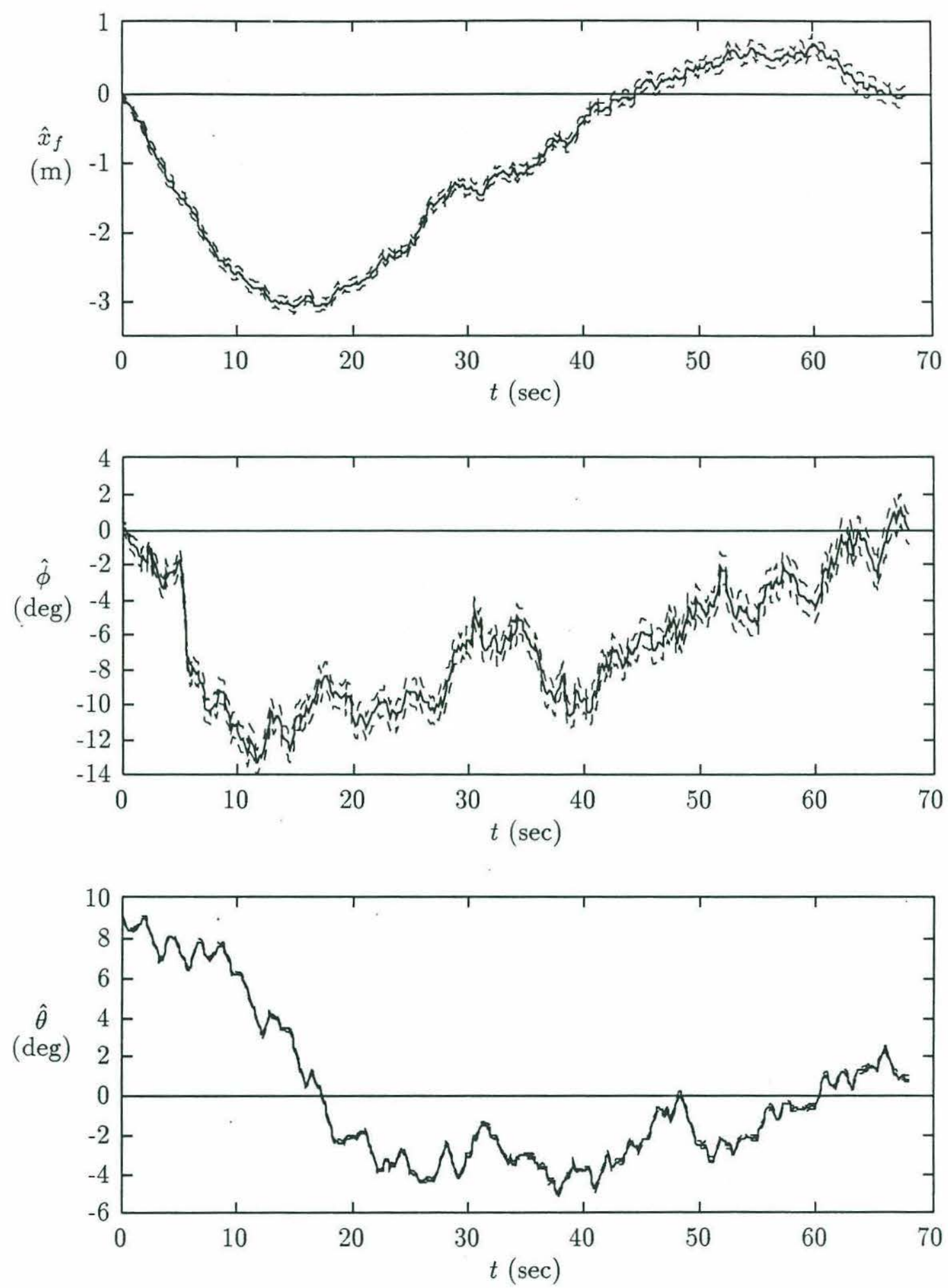

Figure 4.7: Estimates of attitude parameters obtained through the extended least-squares algorithm with $N=4$ and $\beta=1$. 

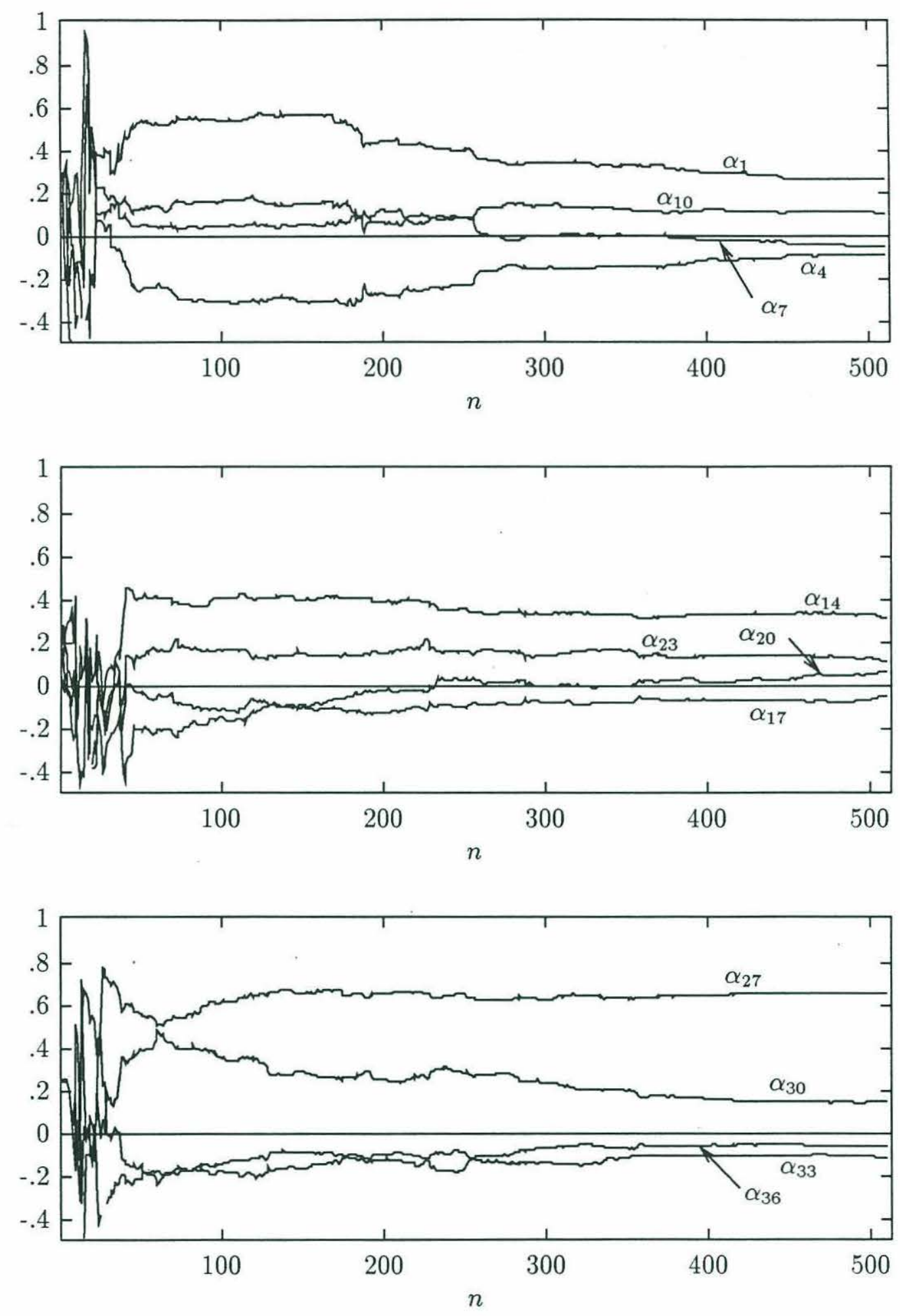

Figure 4.8: Estimates of state-space model parameters obtained through the extended least-squares algorithm with $N=4$ and $\beta=1$. These figures show the evolution of the estimates of the AR coefficients for $\Delta x_{\circ}^{\prime}[n]$ (top), $\Delta y_{o}^{\prime}[n]$ (middle), and $\Delta \theta[n]$ (bottom). For simplicity, the cross-term coefficients are not shown. 
to result from the incorporation of tempcral correlation information on the parameters through the state-space model. Figure 4.8 shovs the estimates of the AR coefficients for $\Delta x_{o}^{\prime}[n], \Delta y_{o}^{\prime}[n]$ and $\Delta \theta[n]$. For simplicity, the cross-term coefficients (i. e., those relating $\Delta x_{o}^{\prime}[n]$ to $\Delta y_{o}^{\prime}[n]$ and $\Delta \theta[n]$, etc.) were not plotted.

Figures 4.9 and 4.10 show the estimates of attitude and model parameters for $N=4$ and $\beta=0.987$ and the same initial estimates used before. In this case, the weight of the observations decreases progressively as they become "old," turning the algorithm into an adaptive Kalman filter capable of responding to variations in the dynamics of the towfish and in the statistics of the observation and process noises. Taking $\beta=0.987$ amounts to applying an exponentially decreasing weight to the data, with a time constant of $10 \mathrm{sec}$. The estimates of attitude parameters in this case do not differ much from those obtained with $\beta=1$. However, the confidence intervals are slightly wider because the estimation at each point no longer takes equally into account all previous observations, but gradually "forgets" old observations. The estimates of the AR model parameters are shown in Fig. 4.10.

The estimates of $\Delta x_{o}^{\prime}[n], \Delta y_{o}^{\prime}[n]$ and $\Delta \theta[n]$ obtained in this chapter will be used in Chapter 5 for correcting the geometric distortions in the sonograph. 

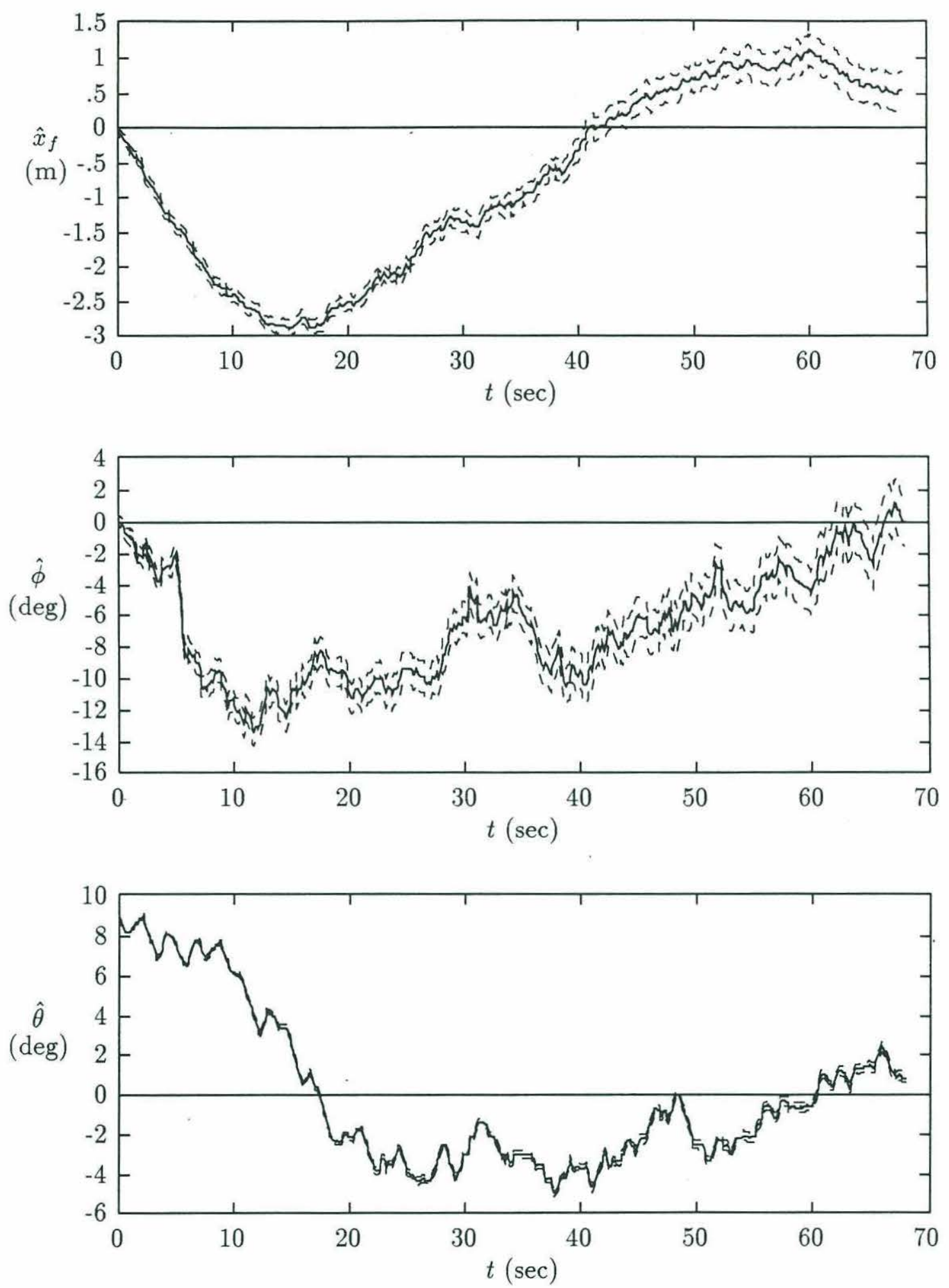

Figure 4.9: Estimates of attitude parameters obtained through adaptive Kalman filtering with $N=4$ and $\beta=0.987$. 

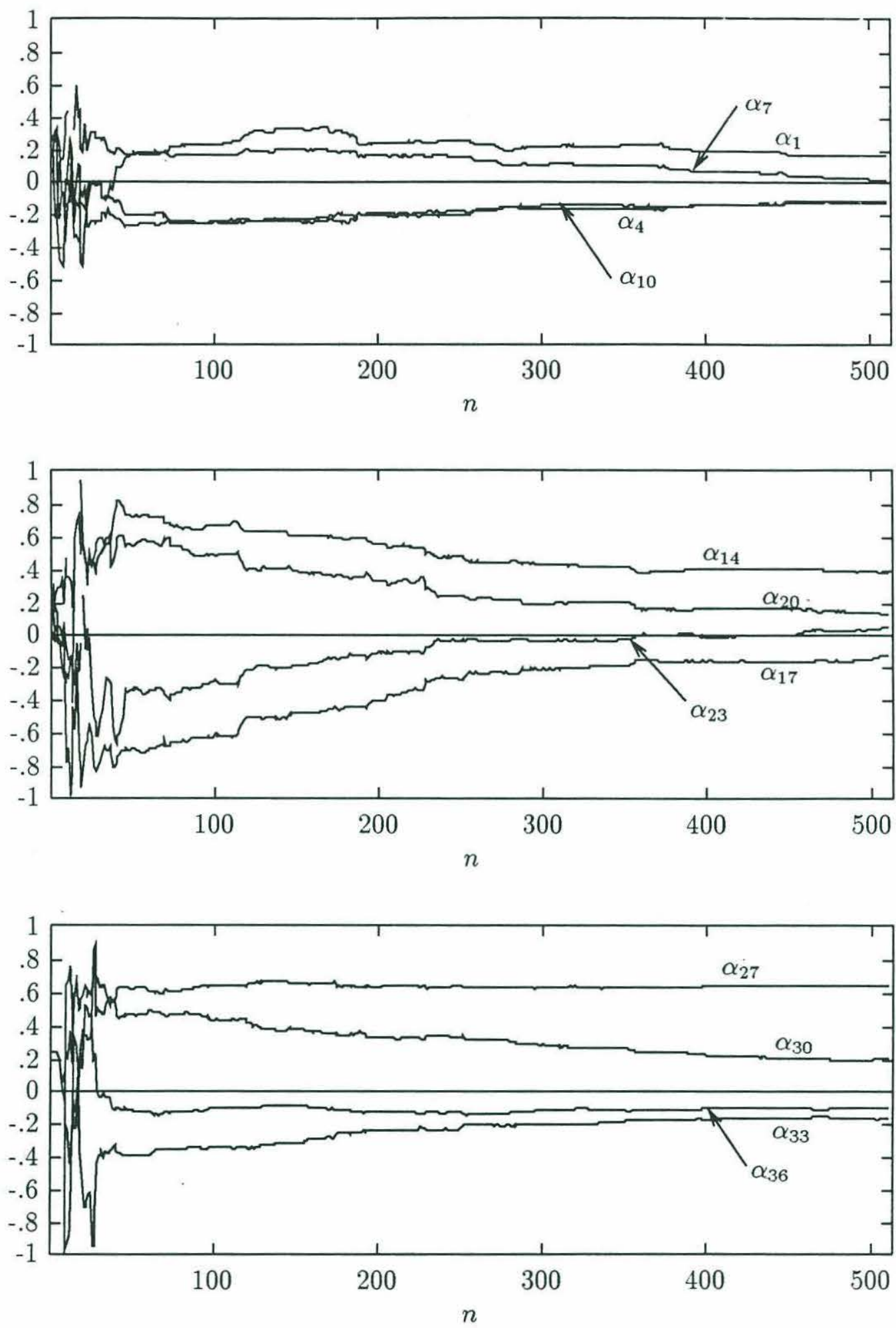

Figure 4.10: Estimates of state-space model parameters obtained through the extended least-squares algorithm with $N=4$ and $\beta=0.987$. These figures show the evolution of the estimates of the AR coefficients for $\Delta x_{o}^{\prime}[n]$ (top), $\Delta y_{o}^{\prime}[n]$ (middle), and $\Delta \theta[n]$ (bottom). For simplicity, the cross-term coefficients are not shown. 


\section{Chapter 5}

\section{Correction of Geometric Distortions}

The distortion parameters estimated through the technique described in Chapter 4 are now used for correcting the geometric distortions in the sonograph. In this final step the sonograph is resampled so that the coordinates of a point in the processed image correspond more closely to its true location on the seabed, assuming the estimates of distortion parameters are accurate. Visual inspection of the corrected sonograph reveals considerable reduction of the geometric distortions. A simulation is carried out to provide further, and more objective, evidence of the efficacy of our technique for estimating and correcting geometric distortions in sonographs.

\subsection{Correction of Cross-Track Distortions}

As indicated in Section 3.2, the slant-range distortion in the sonograph has to be corrected before the sampling displacements $\Delta_{n} x_{s}^{\prime}[l, n]$ can be estimated. This is accomplished by resampling the original sonograph, $s[m, n]$, whose $m$ coordinate corresponds to range to the towfish, to obtain a new image $\bar{s}[l, n]$, whose $l$ coordinate corresponds to horizontal distances on the seabed plane.

Let $s_{i}(r, n]$ denote the sonograph after interpolation along each line. ${ }^{1}$ The slant-range

\footnotetext{
${ }^{1}$ The combination of parenthesis and square bracket is used to indicate that $r$ is a continuous variable, while $n$ is a discrete variable.
} 


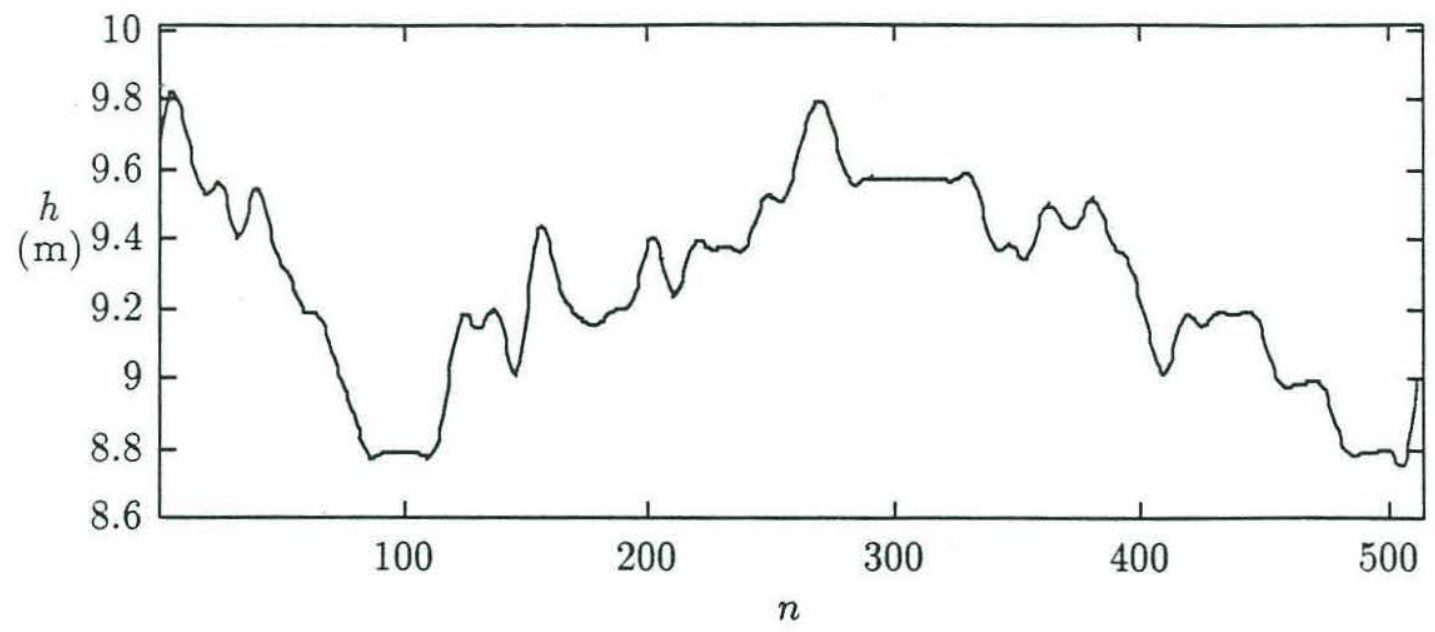

Figure 5.1: Height of the water column measured from the sonograph after lowpass filtering to eliminate discontinuities caused by quantization.

corrected image is given by

$$
\bar{s}[l, n]=\left.s_{i}(r, n]\right|_{r=\sqrt{l^{2}+\left(h[n] /\left(c T_{s} / 2\right)\right)^{2}}},
$$

where $h[n]$ is the distance between the towfish and point $\left(x_{o}[n], y_{\circ}[n]\right)$, as shown in Fig. 2.4, and can be measured from the sonograph as the height of the water column at each line. The interpolation is carried out by multiplying the discrete Fourier transform of the line by a phase shift corresponding to the desired sampling location, before calculating the inverse transform. However, other techniques, such as truncated-sinc or cubic-spline interpolation, may be used instead. If $S_{n}[k], k=0,1, \ldots, N_{m}-1$ denotes the DFT of the $n$th line of $s[m, n]$, we have

$$
\bar{s}[l, n]=\sum_{k=0}^{N_{m}-1} S_{n}[k] e^{\frac{2 \pi}{N_{m}} \sqrt{l^{2}+\left(h[n] /\left(c T_{s} / 2\right)\right)^{2}}} .
$$

Figure 5.1 shows $h[n]$ for the sonograph of Fig. 1.2 after lowpass filtering to eliminate the discontinuities resulting from the quantization of the width of the water column to an integer number of pixels. The slant-range corrected sonograph was shown in Fig. 3.4. From it, the estimates of $\Delta_{n} x_{s}^{\prime}[l, n]$ are calculated.

As discussed in Section 3.3, before the correlation lengths can be calculated, it is necessary to shift the lines of the slant-range corrected image, $\bar{s}[l, n]$, to obtain a new 
image, $\overline{\bar{s}}[l, n]$, for which the sampling displacements $\Delta_{n} x_{s}^{\prime}[l, n]$ are zero. According to the linear model of Section 2.2.3, at each line of the image we have $\Delta_{n} x_{s}^{\prime}[l, n]=\Delta x_{0}^{\prime}[n]$. Therefore, to make the $\Delta_{n} x_{s}^{\prime}[l, n]$ equal to zero, it is enough to shift line $(n+1)$ of the image by $-\widehat{\Delta x_{o}^{\prime}}[l, n] /\left(c T_{s} / 2\right)$ with respect to line $n$, for $n=0,1, \ldots, N_{n}-1$. Equivalently, the $n$th line of the image has to be shifted by $\sum_{i=0}^{n-1} \widehat{\Delta x_{o}^{\prime}}[i] /\left(c T_{s} / 2\right)$ with respect to line number zero. That can be done by multiplying the DFT of the $n$th line, which is denoted by $\bar{S}_{n}[k]$, by the appropriate phase shift, and then taking the inverse transform. Thus,

$$
\overline{\bar{s}}[l, n]=\mathcal{F}^{-1}\left\{\bar{S}_{n}[k] e^{-\frac{2 \pi}{N_{l}} k \sum_{i=0}^{n-1} \widehat{\Delta x_{0}^{\prime}}[i] /\left(c T_{s} / 2\right)}\right\} .
$$

The estimates $\widehat{\Delta x_{0}^{\prime}}[n]$ used in this operation are those obtained through deterministic least-squares, i. e., they are simply the average of the estimates of sampling displacements, $\widehat{\Delta_{n} x_{s}^{\prime}}[l, n]$ for each line of the image. If one wishes to estimate the distortion parameters through adaptive Kalman filtering, then the $\Delta x_{\circ}^{\prime}[n]$ are later re-estimated with the other two distortion parameters, $\Delta y_{0}^{\prime}[n]$ and $\Delta \theta[n]$, simultaneously, through the extended leastsquares algorithm.

The image $\overline{\bar{s}}[l, n]$ is used for calculating the correlation lengths, $L_{n}^{+}[l, n]$ and $L_{n}^{-}[l, n]$, as described in Section 3.3.

\subsection{Estimating the Sampling Point Coordinates}

After the distortion parameters are estimated through one of the techniques presented in Chapter 4, the slant-range corrected sonograph is resampled for correction of the remaining geometric distortions. That requires obtaining estimates of the sampling coordinates, $x_{s}[l, n]$ and $y_{s}[l, n]$, for each point of the image, from the estimates of distortion parameters, $\widehat{\Delta x_{o}^{\prime}}[n], \widehat{\Delta y_{0}^{\prime}}[n]$ and $\widehat{\Delta \theta}[n]$. Using Eqs. (2.12a) and (2.12b), we have

$$
\begin{aligned}
& \widehat{\Delta_{n} x_{s}^{\prime}}[l, n]=\widehat{\Delta x_{0}^{\prime}}[n] \\
& \widehat{\Delta_{n} y_{s}^{\prime}}[l, n]=\widehat{\Delta y_{0}^{\prime}}[n]+l\left(c T_{s} / 2\right) \widehat{\Delta \theta}[n] .
\end{aligned}
$$

These estimates are then converted from the $\left(x^{\prime}, y^{\prime}\right)$ to the $(x, y)$ coordinate system. From Eqs. (2.13a) and (2.13b) we obtain

$$
x=\left(x^{\prime}-x_{0}[n]\right) \cos \theta[n]-\left(y^{\prime}-y_{0}[n]\right) \sin \theta[n]
$$




$$
y=\left(y^{\prime}-y_{0}[n]\right) \cos \theta[n]+\left(x^{\prime}-x_{0}[n]\right) \sin \theta[n] .
$$

Therefore the sampling displacements may be converted from one coordinate system to the other through

$$
\begin{aligned}
& \widehat{\Delta_{n} x_{s}}[l, n]=\widehat{\Delta_{n} x_{s}^{\prime}}[l, n] \cos \hat{\theta}[n]-\widehat{\Delta_{n} y_{s}^{\prime}}[l, n] \sin \hat{\theta}[n] \\
& \widehat{\Delta_{n} y_{s}}[l, n]=\widehat{\Delta_{n} y_{s}^{\prime}}[l, n] \cos \hat{\theta}[n]+\widehat{\Delta_{n} x_{s}^{\prime}}[l, n] \sin \hat{\theta}[n] .
\end{aligned}
$$

Finally, the estimates of sampling point coordinates are calculated recursively from the estimates of sampling displacements,

$$
\begin{aligned}
& \hat{x}_{s}[l, n+1]=\hat{x}_{s}[l, n]+\widehat{\Delta_{n} x}[l, n] \\
& \hat{y}_{s}[l, n+1]=\hat{y}_{s}[l, n]+\widehat{\Delta_{n} y_{s}}[l, n]
\end{aligned}
$$

starting from estimates of the sampling coordinates of the first line, $\hat{x}_{s}[l, 0]$ and $\hat{y}_{s}[l, 0]$, $l=0, \pm 1, \ldots, \pm\left(N_{l}-1\right)$. Equations (2.11a) and (2.11b) may be used to calculate these coordinates, with $x_{0}[0]=y_{0}[0]=0$ and $\theta[0]=\hat{\theta}[0]$.

A graphic representation of the estimates of sampling coordinates can be provided by plotting the estimated sampling points $\left(\hat{x}_{s}[m, n], \hat{y}[m, n]\right)$ in a mesh. Figure 5.2 shows the resulting mesh plot for our sample sonograph. In producing this figure we used the estimates of distortion parameters obtained through deterministic least-squares estimation in Section 4.2 and plotted only every fourth line and column so the mesh would not be too dense. This plot gives a clear idea of how the sonar beam moved across the bottom on that area, according to the estimates of distortion parameters.

\subsection{Correcting Geometric Distortions}

We now address the issue of correcting the geometric distortions affecting the sonograph. The procedure is divided into two steps: interpolation in the cross-track direction followed by interpolation in the along-track direction. 


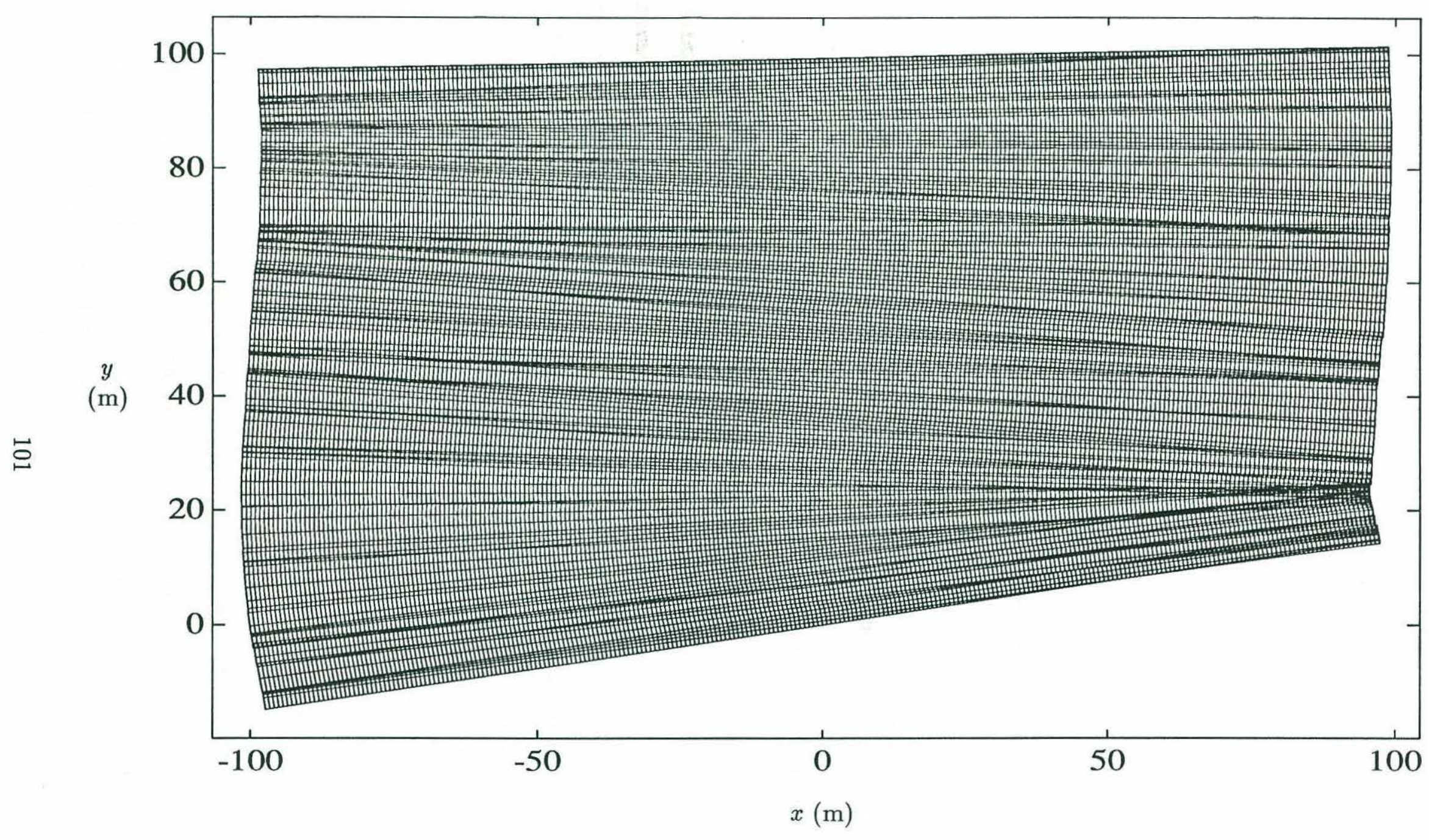

Figure 5.2: Mesh plot illustrating the geometric distortion in the sonograph of Fig. 3.4.

The grid marks the estimated location of the sampling points on the seabed plane. 


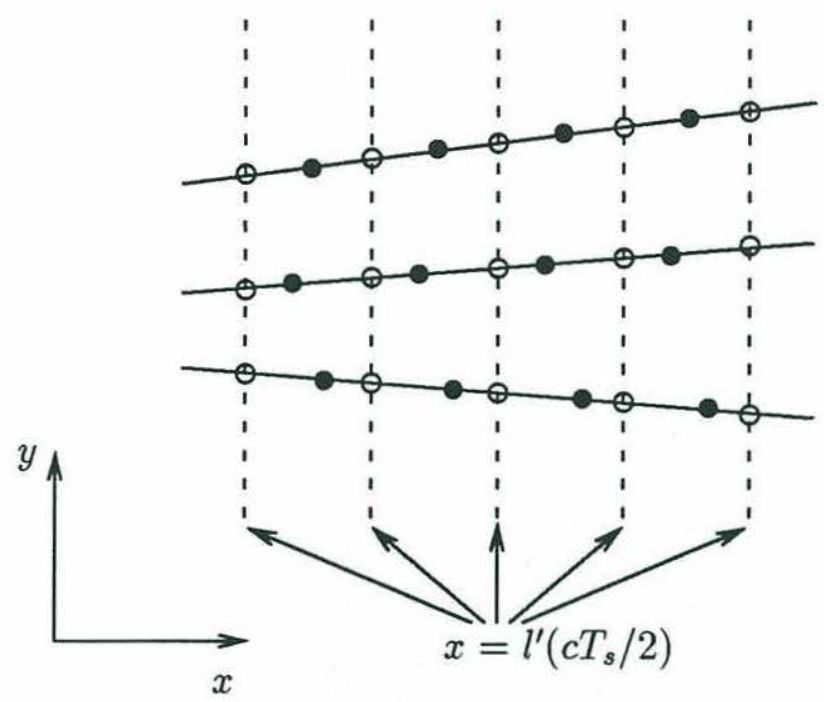

Figure 5.3: Determining the points for interpolation in the cross-track direction. Each line of the image is resampled at points that are equally spaced in the $x$ direction.

\subsubsection{Interpolation in the Cross-Track Direction}

The slant-range corrected image $\bar{s}[l, n]$ is first interpolated to produce a new image $s_{c}\left[l^{\prime}, n\right]$ so its corresponding sampling points on the seabed are equally spaced in the $x$ direction. Figure 5.3 illustrates how the points for interpolating each line of $\bar{s}[l, n]$ are determined. The black dots shown in the figure represent the estimates of the location of sampling points on the seabed plane. Because of the linear model used in Chapter 2, the estimates of sampling points for each sonar pulse fall on a straight line that approximates the trajectory of the sound pulse on the seabed as it travels away from the towfish.

The sampling points of $s_{c}\left[l^{\prime}, n\right]$ are indicated in the image by the uniformly spaced circles whose cross-track coordinates are

$$
x_{c}\left[l^{\prime}\right]=l^{\prime}\left(c T_{s} / 2\right), \quad l^{\prime}=0, \pm 1, \pm 2, \ldots, \pm\left(N_{l^{\prime}}-1\right),
$$

where $N_{l}$ is the desired number of columns on the starboard and port sides of the resampled image. The corresponding distances along the $n$th scan line are denoted by $l_{c}\left[l^{\prime}, n\right]$ and in general are non-integer. They are calculated by finding the two points $\left[l_{1}, n\right]$ and $\left[l_{2}, n\right]$ whose sampling coordinate estimates $\hat{x}_{s}\left[l_{1}, n\right]$ and $\hat{x}_{s}\left[l_{2}, n\right]$ are located 
immediately before and immediately after $x_{c}\left[l^{\prime}\right]$, i. e.,

$$
\hat{x}_{s}\left[l_{1}, n\right] \leq x_{c}\left[l^{\prime}\right]<\hat{x}_{s}\left[l_{2}, n\right], \quad l_{2}=l_{1}+1
$$

and then by computing $l_{c}\left[l^{\prime}, n\right]$ by linearly interpolating the corresponding $l$ coordinates,

$$
l_{c}\left[l^{\prime}, n\right]=l_{1}+\left(l_{2}-l_{1}\right) \frac{x_{c}\left[l^{\prime}\right]-\hat{x}_{s}\left[l_{1}, n\right]}{\hat{x}_{s}\left[l_{2}, n\right]-\hat{x}_{s}\left[l_{1}, n\right]} .
$$

In the next subsection, we will need to know the along-track coordinates of these points, which we denote by $y_{c}\left[l^{\prime}, n\right]$. These are computed by linearly interpolating between the $y$ coordinates of points $\left[l_{1}, n\right]$ and $\left[l_{2}, n\right]$,

$$
y_{c}\left[l^{\prime}, n\right]=\hat{y}_{s}\left[l_{1}, n\right]+\left(\hat{y}_{s}\left[l_{2}, n\right]-\hat{y}_{s}\left[l_{1}, n\right]\right) \frac{x_{c}\left[l^{\prime}\right]-\hat{x}_{s}\left[l_{1}, n\right]}{\hat{x}_{s}\left[l_{2}, n\right]-\hat{x}_{s}\left[l_{1}, n\right]} .
$$

The $n$th line of the slant-range corrected image, $\bar{s}[l, n]$, is then interpolated at points $l_{c}\left[l^{\prime}, n\right], l^{\prime}=0,1, \ldots,\left(N_{l^{\prime}}-1\right)$. The interpolation is again performed by multiplying the DFT of the line by the appropriate phase shift for each sample point, before calculating the inverse transform. Thus, if $\bar{S}_{n}[k], k=0,1, \ldots, N_{l}-1$ denotes the DFT of the $n$-th line of $\bar{s}[l, n]$, we have

$$
s_{c}\left[l^{\prime}, n\right]=\sum_{k=0}^{N_{l}-1} \bar{S}_{n}[k] e^{\frac{2 \pi}{N_{l}} k l_{c}\left[l^{\prime}, n\right]} .
$$

\subsubsection{Interpolation in the Along-Track Direction}

By following the procedure of the last subsection one obtains a new image $s_{c}\left[l^{\prime}, n\right]$, whose corresponding sampling points on the seabed are now correctly located in the $x$ direction, but are still irregularly spaced in the $y$ direction. The final step for correcting the geometric distortions is to resample each column of $s_{c}\left[l^{\prime}, n\right]$ to produce a final image $s_{a}\left[l^{\prime}, n^{\prime}\right]$ whose corresponding sampling points on the seabed plane are regularly spaced in both the $x$ and $y$ directions.

Figure 5.4 illustrates this new situation. The circles shown in that figure are the same as those shown in Fig. 5.3 and mark the location of the sampling points associated with $s_{c}\left[l^{\prime}, n\right]$; the points where each column should be interpolated are marked in the figure by small boxes. Their along-track coordinates are given by

$$
y_{a}\left[n^{\prime}\right]=n^{\prime}\left(v T_{f}\right), \quad n^{\prime}=0,1, \ldots, N_{n^{\prime}}-1,
$$




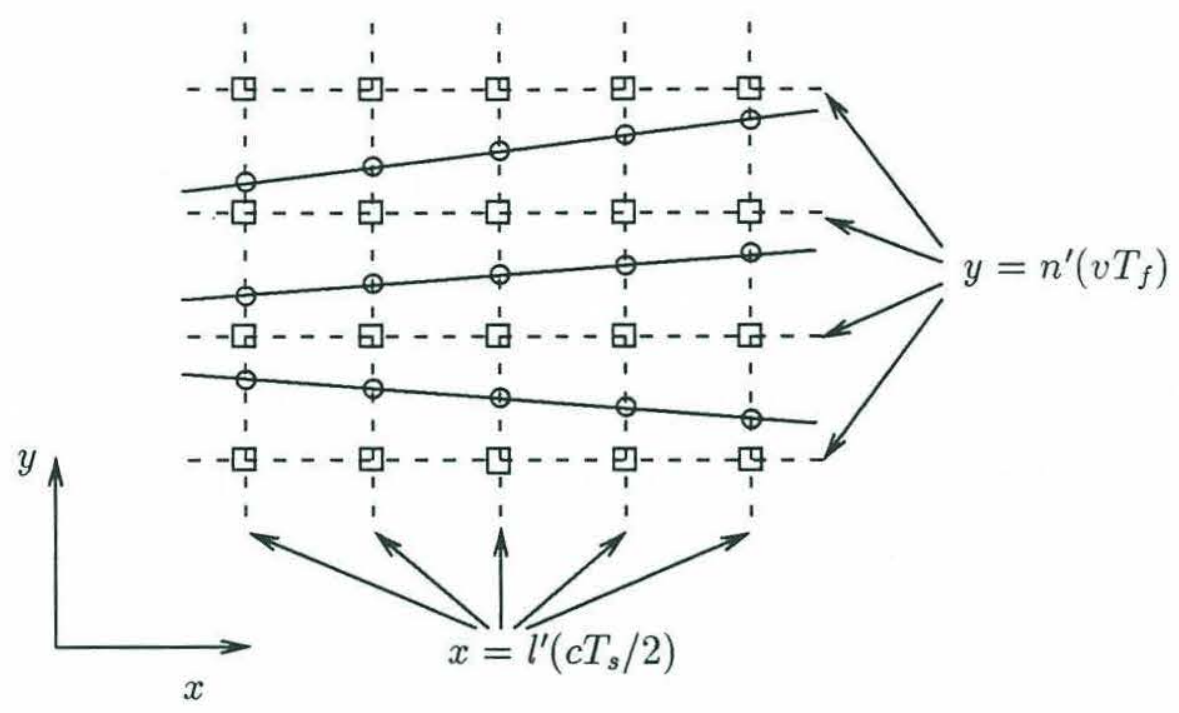

Figure 5.4: Determining the points for interpolation in the along-track direction.

where $N_{n^{\prime}}$ is the desired number of lines in the final image. The corresponding distances along the $l^{\prime}$-th column of $s_{c}\left[l^{\prime}, n\right]$ are denoted by $n_{a}\left[l^{\prime}, n^{\prime}\right]$ and are in general non-integer. Paralleling the procedure of the previous section, these are calculated by finding the two points $\left[l^{\prime}, n_{1}\right]$ and $\left[l^{\prime}, n_{2}\right]$ whose sampling coordinates $y_{c}\left[l^{\prime}, n_{1}\right]$ and $y_{c}\left[l^{\prime}, n_{2}\right]$, calculated through Eq. (5.5) are located immediately before and immediately after $y_{a}\left[n^{\prime}\right]$, i. e.,

$$
y_{c}\left[l^{\prime}, n_{1}\right] \leq y_{a}\left[n^{\prime}\right]<y_{c}\left[l^{\prime}, n_{2}\right], \quad n_{2}=n_{1}+1,
$$

and then by computing $n_{a}\left[l^{\prime}, n^{\prime}\right]$ by linearly interpolating the corresponding $n$ coordinates,

$$
n_{a}\left[l^{\prime}, n^{\prime}\right]=n_{1}+\left(n_{2}-n_{1}\right) \frac{y_{a}\left[n^{\prime}\right]-y_{c}\left[l^{\prime}, n_{1}\right]}{y_{c}\left[l^{\prime}, n_{2}\right]-y_{c}\left[l^{\prime}, n_{1}\right]} .
$$

Before proceeding to interpolate the columns, we need to address a problem that is not present in the cross-track direction. Because of the occurrence of backscanning, the sequence of coordinates $y_{c}\left[l^{\prime}, n\right], n=0,1, \ldots, N_{n}-1$, may not be strictly increasing, i. e., we do not necessarily have $y_{c}\left[l^{\prime}, n_{1}\right]>y_{c}\left[l^{\prime}, n_{2}\right]$ for $n_{1}>n_{2}$. As pointed out in Chapter 1 , backscanned areas appear in triplicate in the image, although the three passes may not look exactly the same due to the different viewing angles. Because of that fact, and because the registration among the three passes may not be accurate enough, attempting 
to merge the three views of a backscanned area during the interpolation of the columns is not likely to yield good results. Instead, we choose to preserve only one view of the backscanned area. To achieve greater image continuity, it is better to select either the first or the third pass, since the middle pass is isolated from both the preceding and the forthcoming portions of the sonograph. Furthermore, the beam is likely to scan the bottom more slowly during the first pass, when it is de-accelerating to reverse its direction of movement, than during the third pass when, after reversing direction a second time, it is accelerating to compensate for the time lost during backscanning. Therefore, we select only the first pass over backscanned areas. In practice, that amounts to considering only progressively increasing values of $y_{c}\left[l^{\prime}, n\right]$ when looking for the interval surrounding the interpolating coordinate $y_{a}\left[n^{\prime}\right]$.

Resampling in the along-track direction is again accomplished by multiplying the DFT of each column of $s_{c}\left[l, n^{\prime}\right]$ by the appropriate phase shift for each point before computing the inverse transform. Denoting by $S_{l^{\prime}}[k], k=0,1, \ldots, N_{n}-1$, the DFT of the $l^{\prime}$ th column of $s_{c}\left[l^{\prime}, n\right]$, we have

$$
s_{a}\left[l^{\prime}, n^{\prime}\right]=\sum_{k=0}^{N_{n}-1} S_{l^{\prime}}[k] e^{\frac{2 \pi}{N_{n}} k n_{a}\left[l^{\prime}, n^{\prime}\right]} .
$$

Figure 5.5 shows the sonograph of Fig. 1.2 after correction of the geometric distortions as described in this section. The estimates of distortion parameters used in estimating the sampling coordinates are those obtained through deterministic least-squares estimation in Chapter 4. The lower right corner of the image is shown enlarged in Fig. 5.6 alongside the corresponding area of the original sonograph. Notice how the cable lying on the bottom that appeared pronouncedly jagged as a result of yawing now presents a slowly varying curvature that is likely closer to its true shape. Notice also how the multiple images of objects in backscanned areas are correctly replaced by single images of those objects. However, some areas of the corrected image in Fig. 5.5 appear slightly blurred, as a result of aliasing during the acquisition of the original sonograph. In fact, perfect reconstruction of the image is not possible in areas where the sampling rate in the alongtrack direction is greater than the Nyquist rate of the backscattering function $b(x, y)$, as a result of faster motion of the sonar beam. 


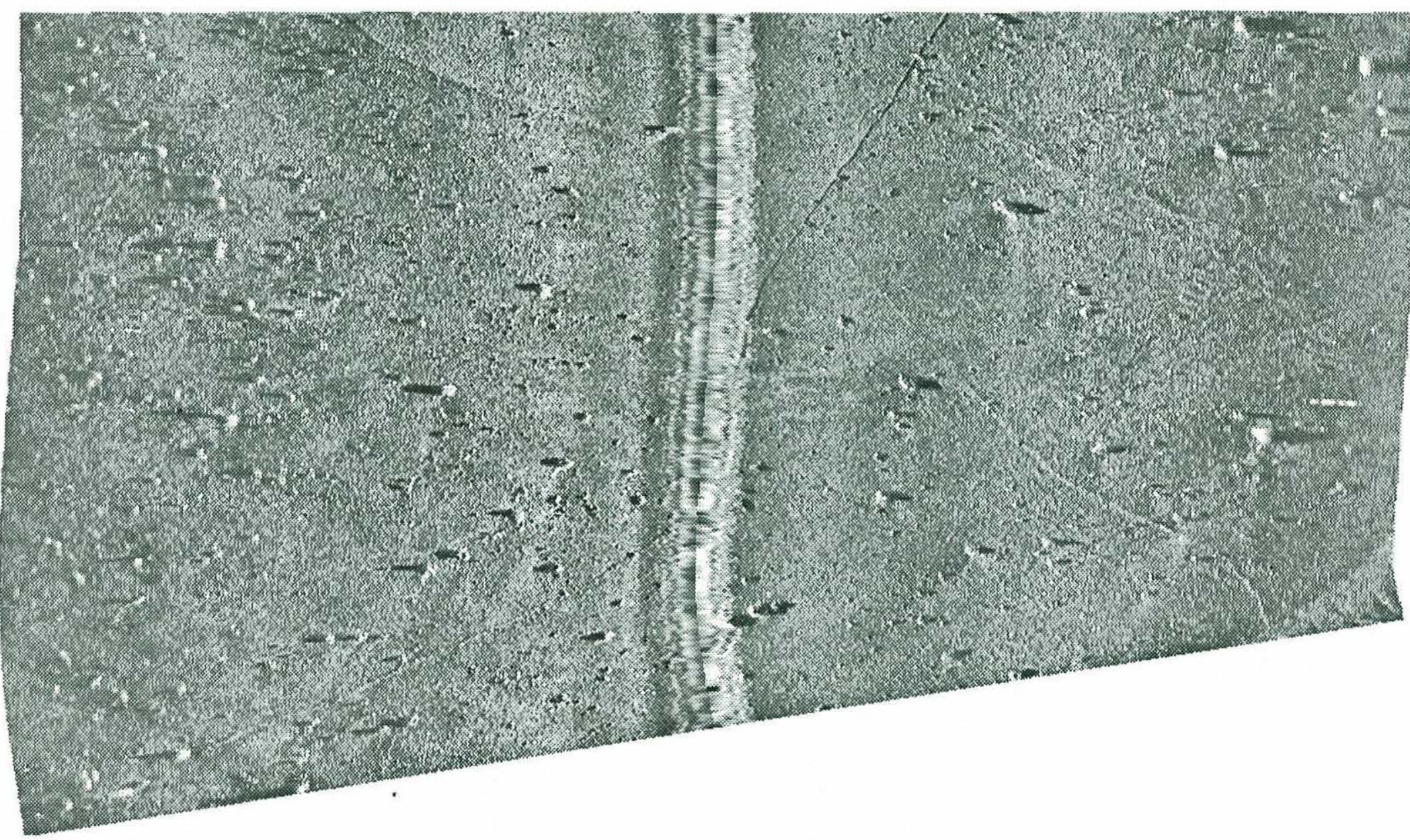

Figure 5.5: Sonograph of Fig. 1.2 after correction of geometric distortions. 

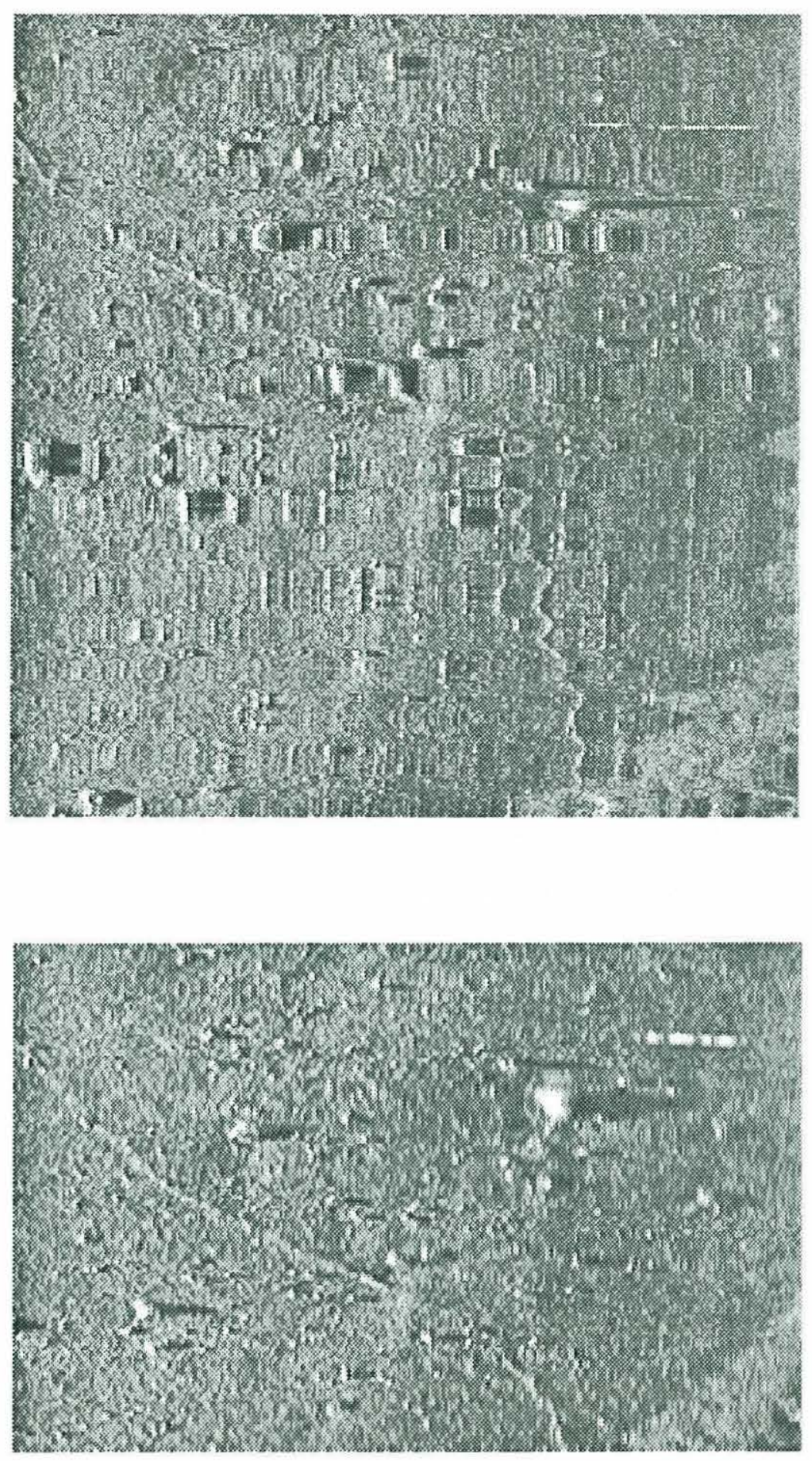

Figure 5.6: Comparison of a detail of the original and corrected sonographs. Correction of geometric distortions eliminates the jagged appearance of the cable and the multiple images of rocks, caused by backscanning. 


\subsection{Simulation}

The side-scan sonar employed for making the sonographs used in this thesis was a common commercial unit and was not equipped with attitude sensors. Therefore, measurements of the true values of the attitude parameters are not available for comparison with the estimates obtained in Chapter 4. As seen in Section5.3, visual inspection of the sonograph after correction of the geometric distortions provides strong subjective indication of the efficacy of our algorithm. However, a more objective evaluation can be provided by a simulation.

Simulating geometric distortions requires an undistorted image of the seabed that can be resampled in an irregular pattern to simulate the effect of towfish instabilities. Since all the sonographs in our data set present some degree of geometric distortion, it is necessary to artificially generate an image on which to perform the simulation. The sonograph of Fig. 5.5 itself could be used to that end, provided that application of the algorithm of Chapters 3 and 4 to that corrected image indicates that it is free of geometric distortions. However, such is not the case, because of the occurrence of aliasing in the original sonograph. As explained in Section 5.3, in areas where the original sonograph is aliased, the corrected image appears blurred, which causes the correlation lengths to be longer than in areas free of aliasing. As a result, the algorithm indicates that the corrected image is still affected by geometric distortions.

To obtain the desired image for the simulation, it is necessary to circumvent the problem presented by aliased areas. In Chapter 4 the distortion parameters for each line of the sonograph were estimated from the side that had smaller estimated sampling intervals $\Delta_{n} y_{s}^{\prime}[l, n]$. That situation was illustrated in Fig. 4.1. In that example, when the geometric distortions are corrected the sampling displacements on the port side $(l<0)$ are made larger to fit the linear model represented by the straight line. That results in the corrected image appearing blurred on the port side at that line, as explained above. That effect can be avoided if the distortion parameters are estimated separately from each side of the line, as indicated in Fig. 5.7. Figure 5.8 shows the result of processing each side of the sonograph with estimates of distortion parameters obtained from that 


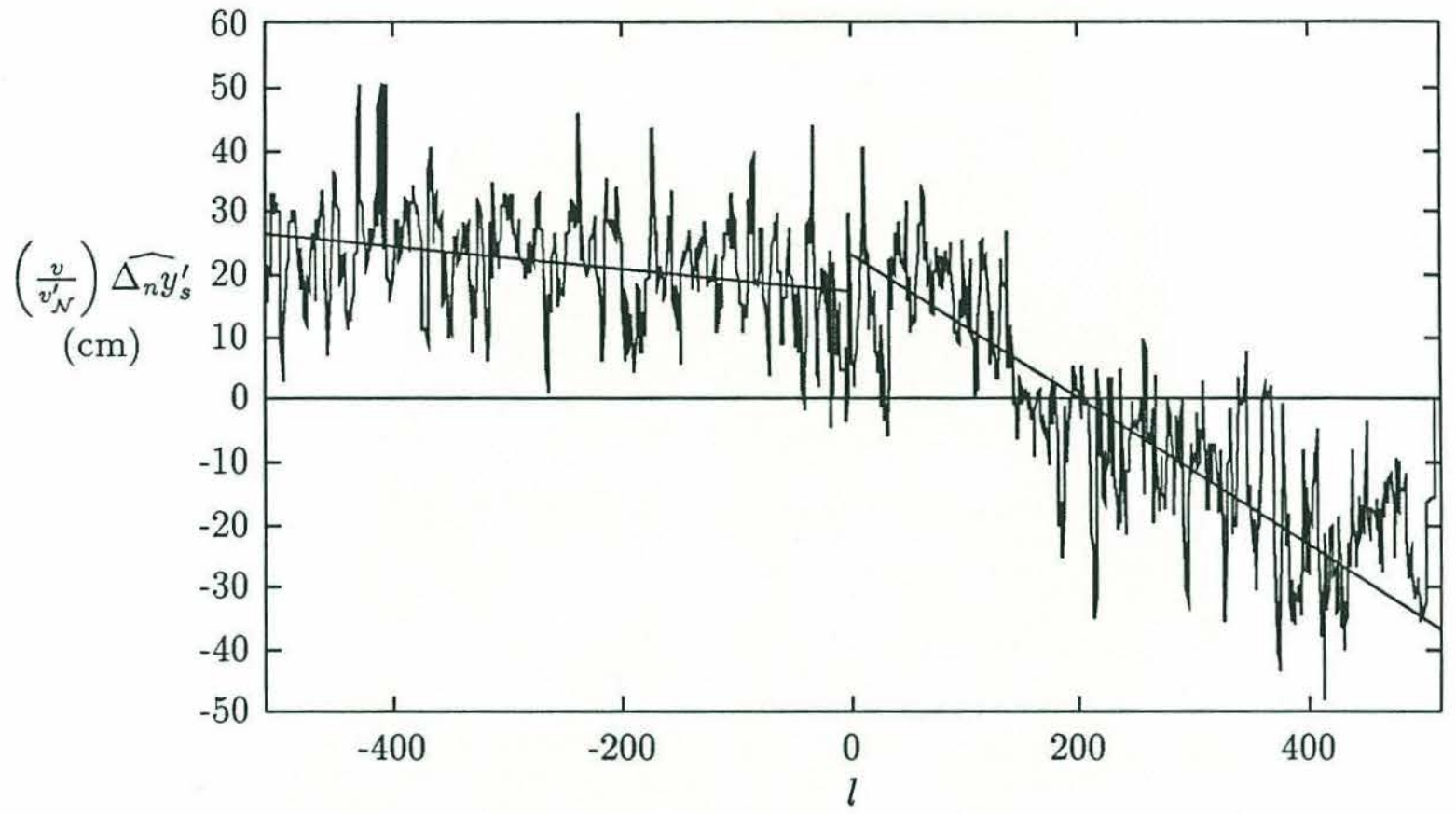

Figure 5.7: An image unaffected by aliasing-related blurring can be obtained by estimating the distortion parameters separately from each side of the sonograph, as illustrated in this example.

side only. The rectangular areas outlined in each image are then cut out and spliced side by side to produce the image in Fig. 5.9, that can be used for the simulation.

The image of Fig. 5.9 is now resampled on an irregular grid to simulate the production of a sonograph affected by geometric distortions. The simulated distortion parameters are generated by passing white Gaussian noise through an autoregressive model with 4 poles of magnitude 0.8 and phases $\pm \pi / 8$ and $\pm \pi / 16$ radians. The location of the poles and the energy of the input noise were chosen so that the resulting simulated parameters had characteristics similar to those of the distortion parameters estimated from the original sonograph. Figure 5.10 shows the undistorted image and the simulated distorted sonograph. The figure also shows the simulated sonograph after correction of the slant-range distortion and detection of backscanning.

Figure 5.11 shows the simulated attitude parameters, along with the estimates obtained through deterministic least-squares estimation, adaptive Kalman filtering, and 

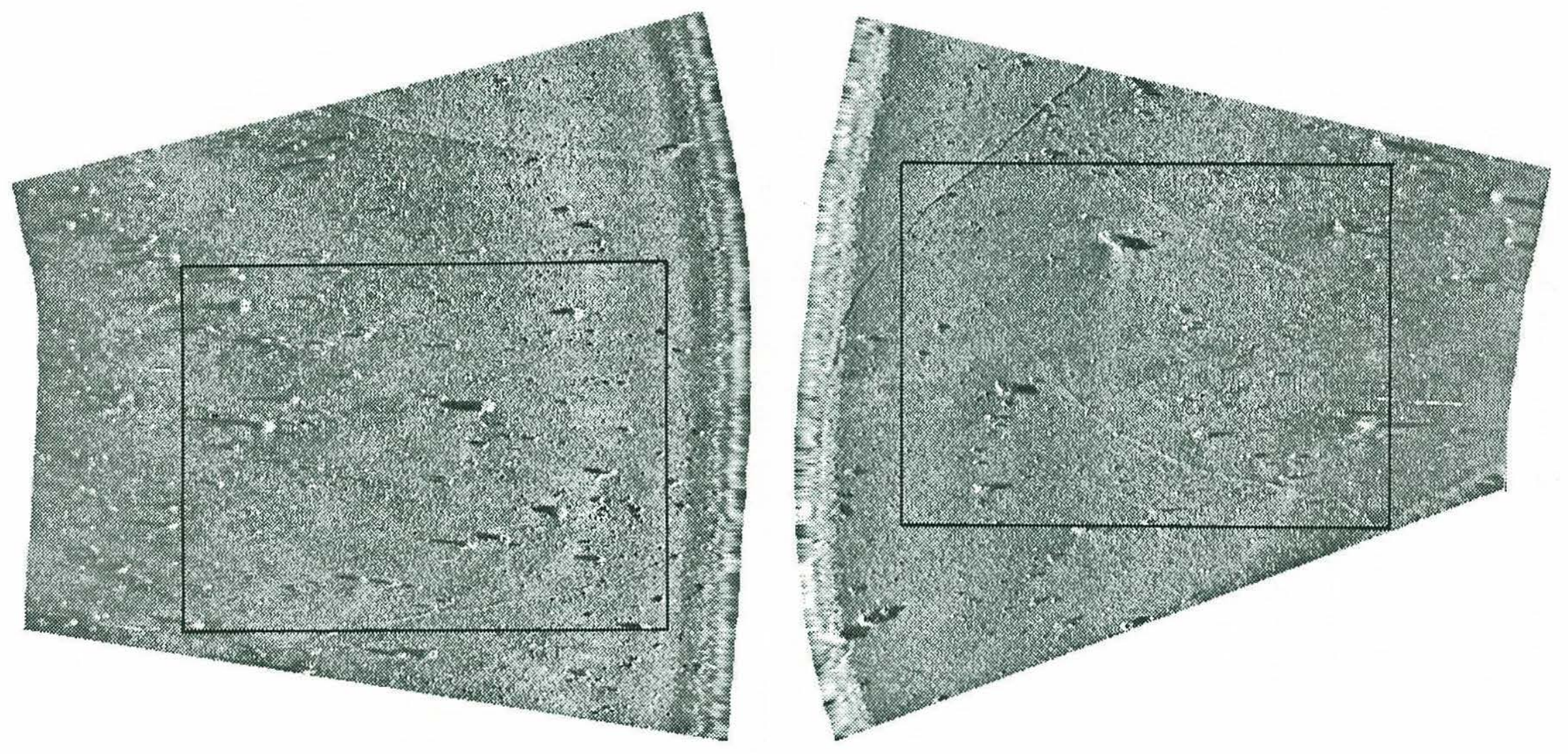

Figure 5.8: Result of independently processing the starboard and port sides of the sonograph, to avoid blurring of aliased areas. 


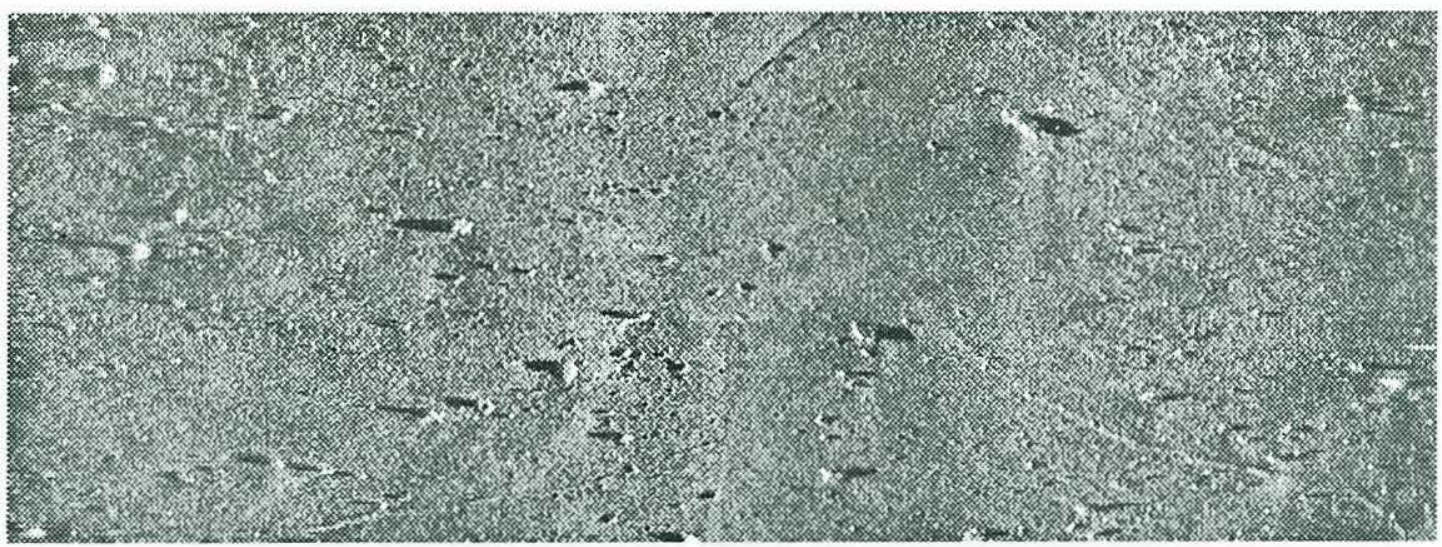

Figure 5.9: Image used in the simulation. It was formed by joining the rectangular sections outlined in the images of Fig. 5.8.

with a Kalman filter using the true state-space model employed to generate the simulated distortion parameters. The estimates obtained through adaptive Kalman filtering do not differ significantly from those obtained through deterministic least-squares estimation, except for a smaller high-frequency content. As noted in Chapter 4, this is because all the autoregressive parameters are being estimated concurrently with the distortion parameters and, consequently, the state-space model contributes little a priori information. In fact, the estimates of state-space model parameters, $\underline{\hat{\alpha}}[n]$, obtained through the adaptive Kalman filter failed to converge to the true values. However, if the true state-space model is used in a standard Kalman filter, the resulting estimates show a noticeable improvement, as seen in the figure.

Table 6.1 presents the maximum absolute estimation errors for the deterministic least-squares (DLS), adaptive Kalman filter (AKF), and standard Kalman filter (KF) algorithms. Notice that using the true state-space model in the standard Kalman filter reduces the maximum absolute estimation error of all three parameters by approximately $50 \%$ with respect to deterministic least-squares estimation and adaptive Kalman filtering. The estimates of yaw angle obtained through any of the three algorithms are remarkably accurate, with the standard Kalman filter yielding a maximum absolute estimation error of less than one degree. The estimates presented here were obtained with $p=140$. Smaller estimation errors may be expected from all algorithms with a larger number of 

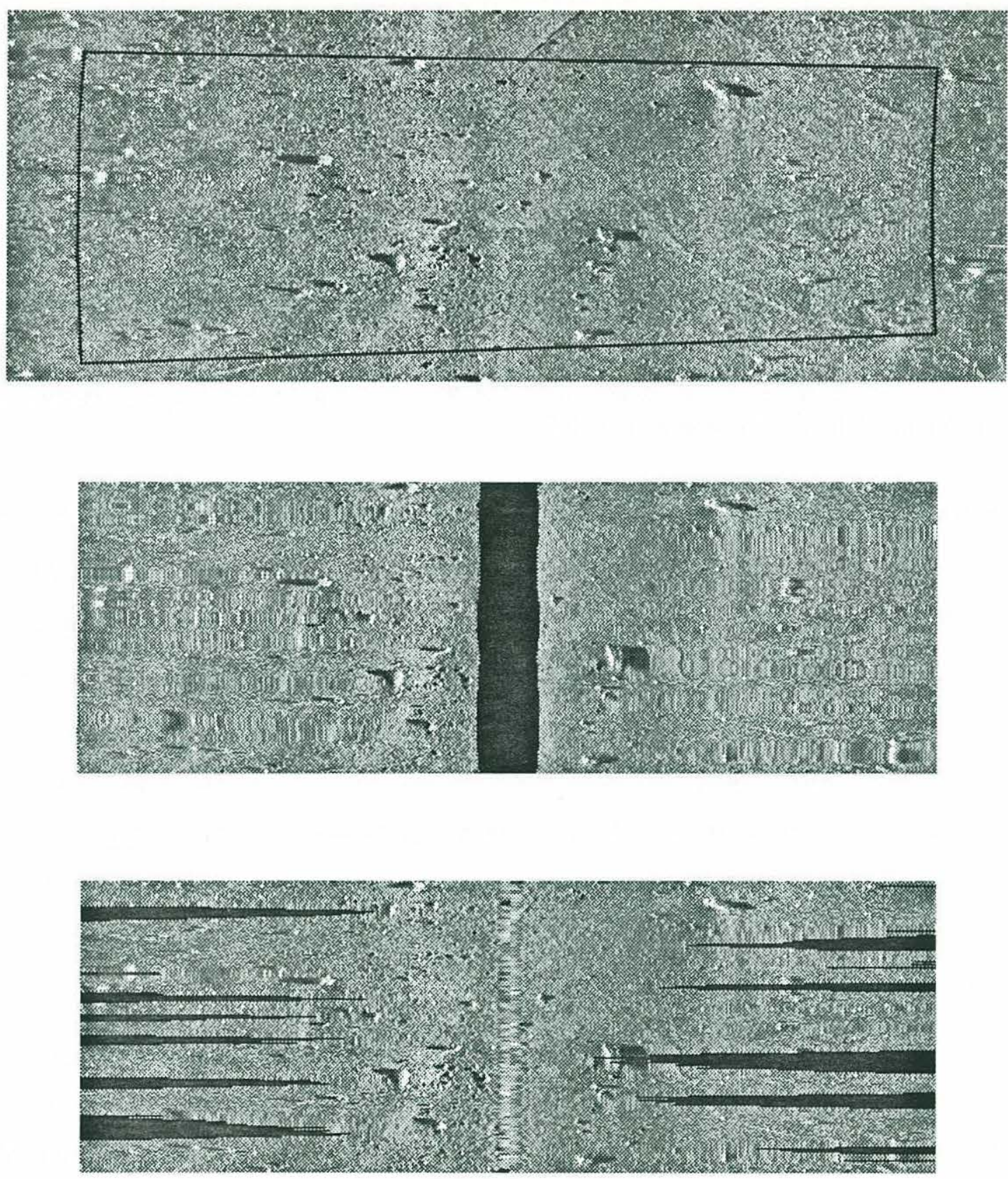

Figure 5.10: The area outlined in the undistorted image (top) is resampled as if scanned by a side-scan sonar subject to motion instabilities, to produce a simulated distorted sonograph (middle). The bottom image is the simulated sonograph after correction of the slant-range distortion and detection of backscanning. 

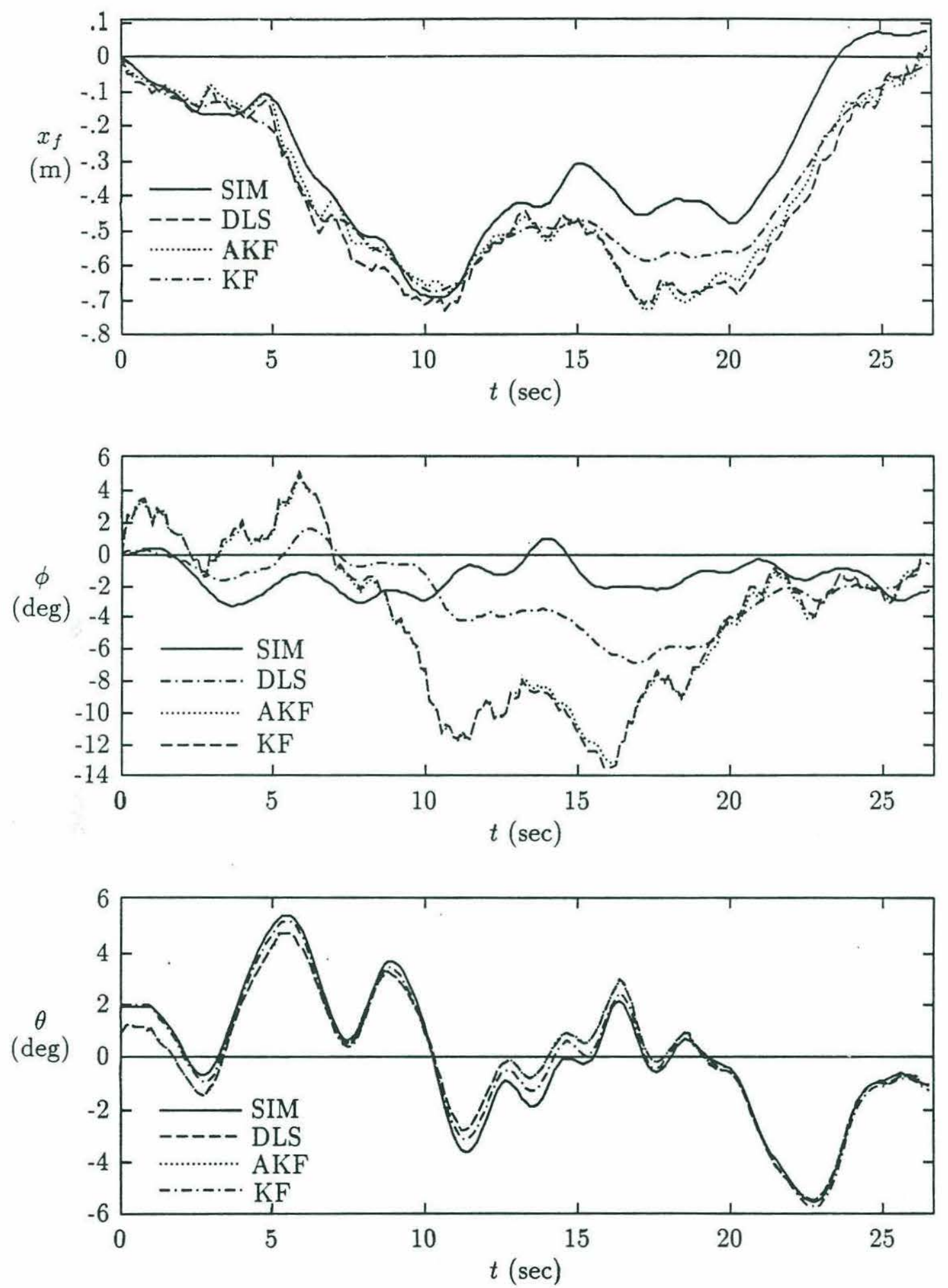

Figure 5.11: Simulated attitude parameters (SIM) and estimates obtained through deterministic least-squares estimation (DLS), adaptive Kalman filtering (AKF), and standard Kalman filtering (KF). 
Table 5.1: Maximum absolute estimation errors.

\begin{tabular}{|c|c|c|c|}
\hline Algorithm & $x_{f}$ & $\phi$ & $\theta$ \\
\hline DLS & $26.87 \mathrm{~cm}$ & $11.37^{\circ}$ & $1.18^{\circ}$ \\
AKF & $29.25 \mathrm{~cm}$ & $11.03^{\circ}$ & $1.19^{\circ}$ \\
KF & $18.84 \mathrm{~cm}$ & $4.84^{\circ}$ & $0.66^{\circ}$ \\
\hline
\end{tabular}

observation points.

The results obtained with the adaptive Kalman filter and with the standard Kalman filter correspond to extreme cases in which either none of the autoregressive model parameters are known or all of them are known, respectively. Intermediate values of maximum estimation errors, as well as better estimates of the unknown model parameters, can be expected from the adaptive Kalman filter if only a few of the autoregressive model parameters are unknown.

Figure 5.12 shows the undistorted image along with the simulated sonograph and the reconstructed image. Comparison of the shapes of rocks and scours in the three images shows that the algorithm achieves a significant reduction of the simulated geometric distortions. As pointed out before, perfect reconstruction is not possible in areas where the sampling interval was large enough to cause aliasing.

\subsection{Further Examples}

A second sonograph from our data set is shown in Fig. 5.13, and in Fig. 5.14 after correction of the slant-range distortion and backscanning detection. The corrected sonograph is shown in Fig. 5.15. The correction of geometric distortions is most evident in the upper right-hand corner of the image, where features on the bottom appear pronouncedly stretched in the original sonograph, but acquire more natural shapes after processing. The estimates of attitude parameters obtained from that sonograph through deterministic least-squares estimation are shown in Fig. 5.16.

A third example is given in Figs. 5.17 through 5.19. As in the first two examples, the jagged appearance of the cable seen in the original sonograph is made consider- 

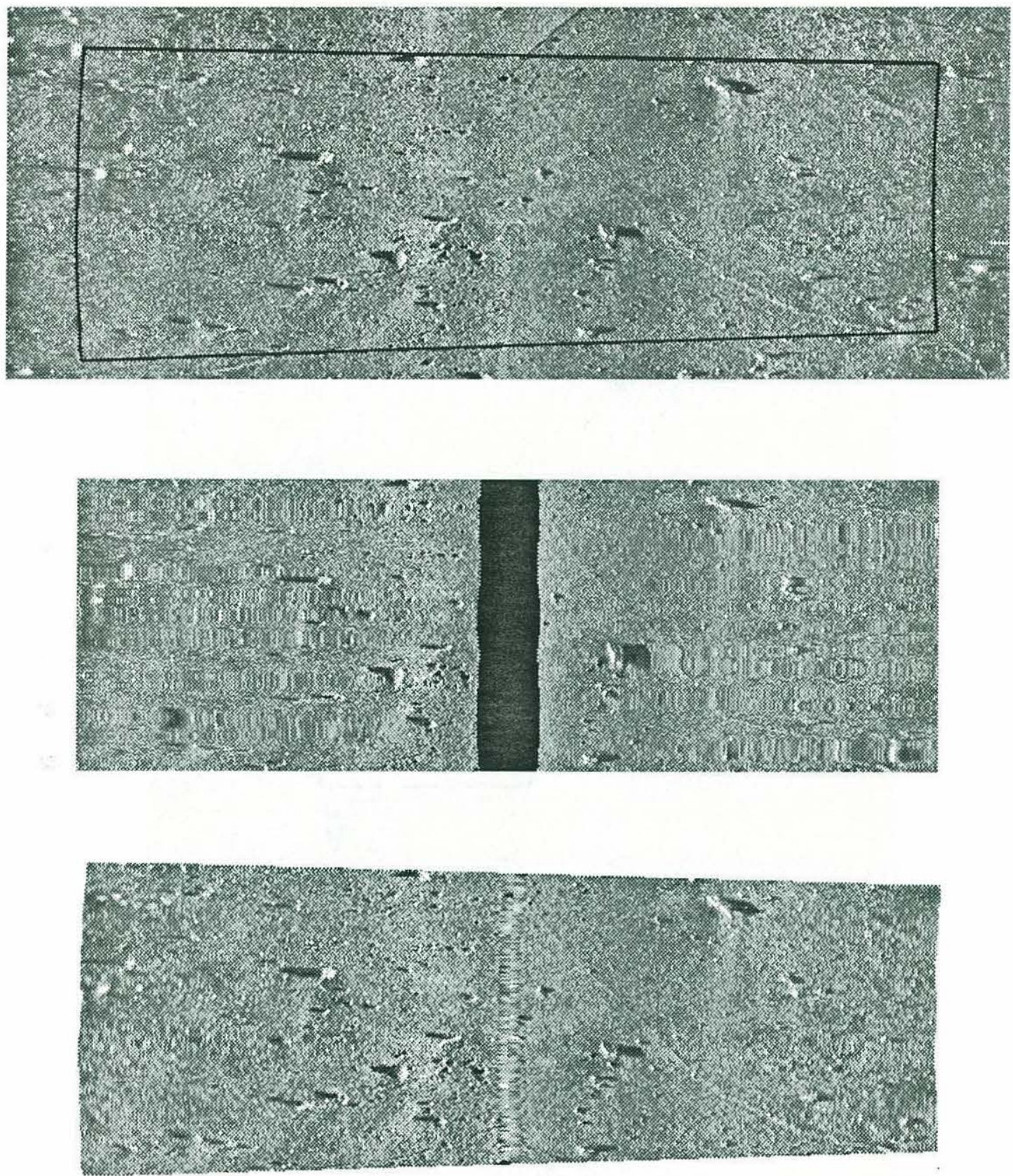

Figure 5.12: Comparison of the original image (top), the simulated sonograph (middle) and reconstructed image (bottom) reveals that the algorithm is capable of effectively reducing geometric distortions. 


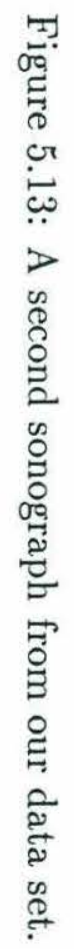

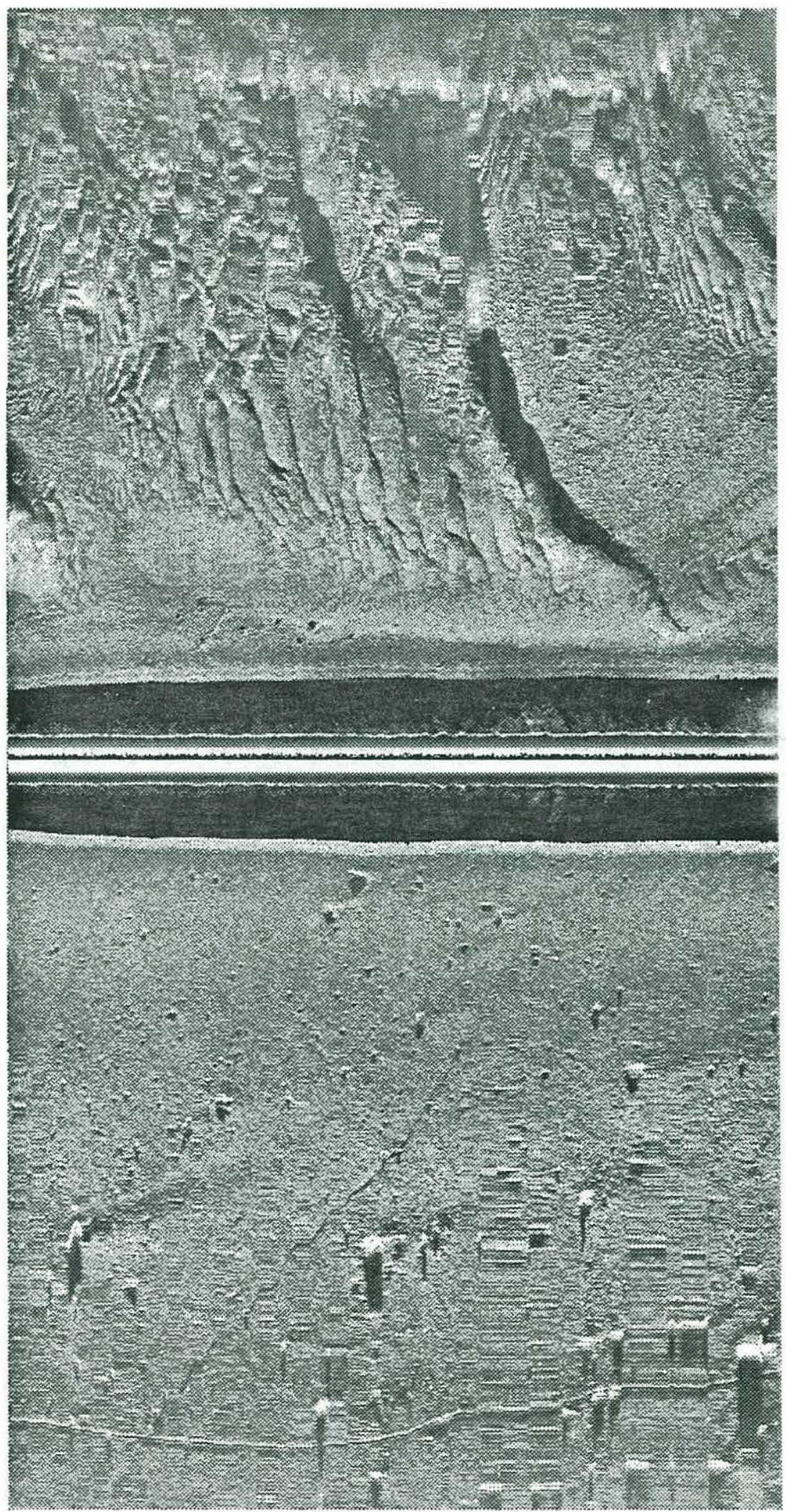




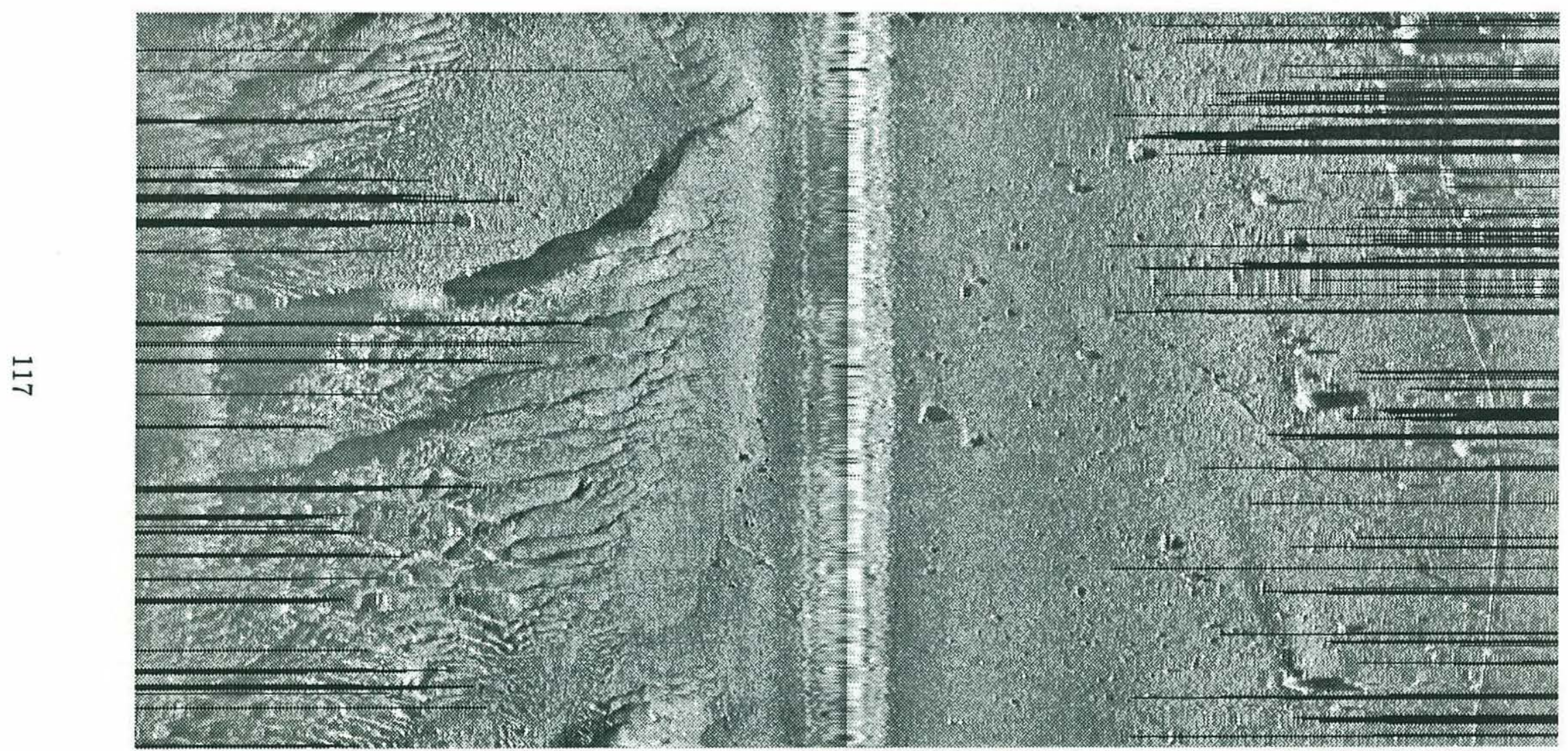

Figure 5.14: Sonograph of Fig. 5.13 after correction of the slant-range distortion and backscanning detection. 


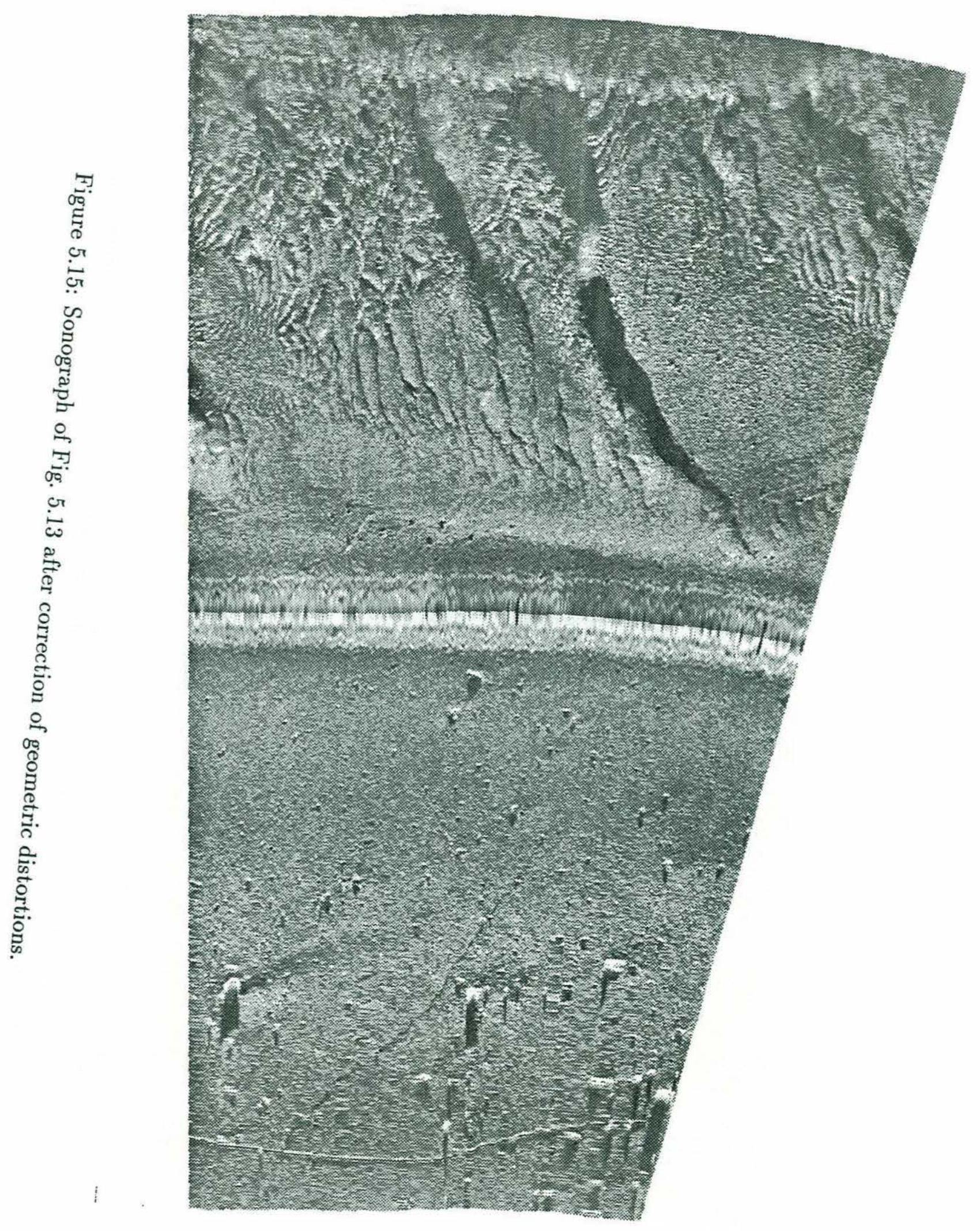



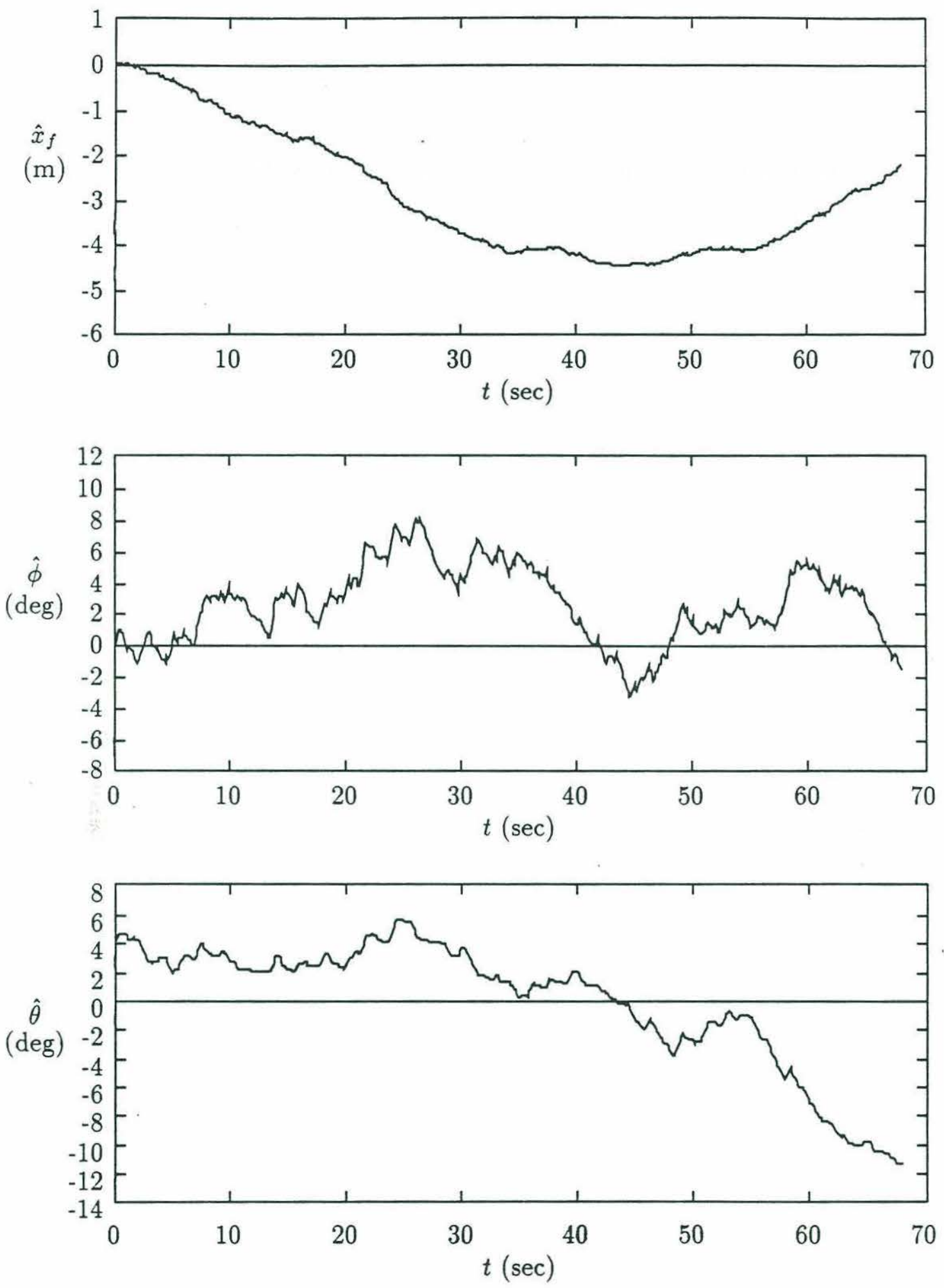

Figure 5.16: Estimates of attitude parameters for the sonograph of Fig. 5.13. 
ably smoother by the correction of geometric distortions. Several rocks that appear compressed, stretched, or with multiple images in the original sonograph acquire more natural shapes after processing, which are likely closer to their true shapes on the seabed.

Geometrically corrected sonographs, such as those shown in Figs. 5.5, 5.15, and 5.19, are the end product of the techniques presented in this thesis. The next chapter presents final considerations. 

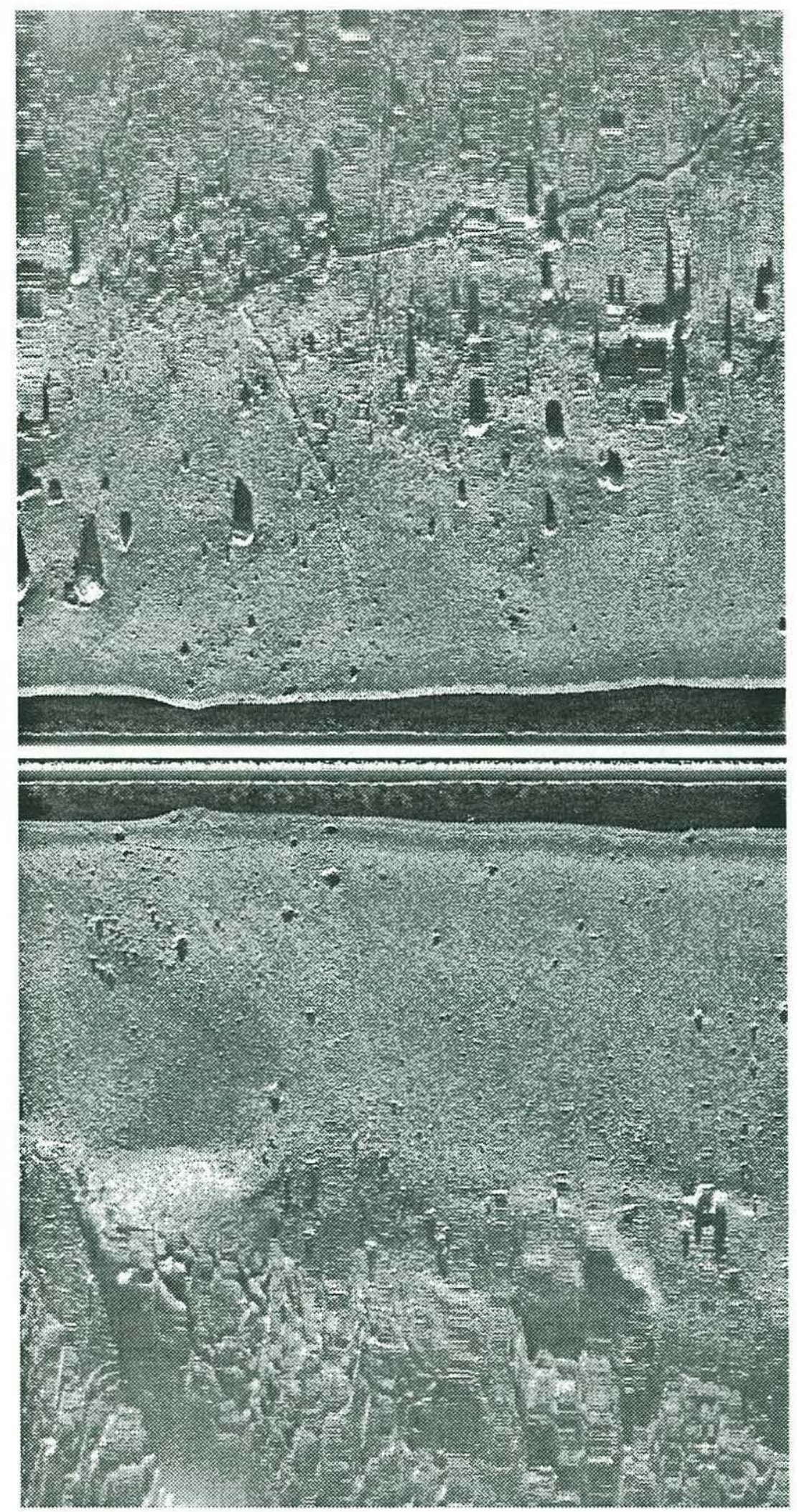

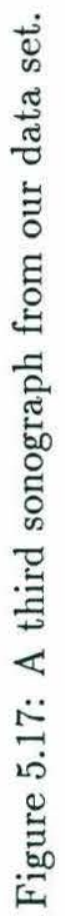




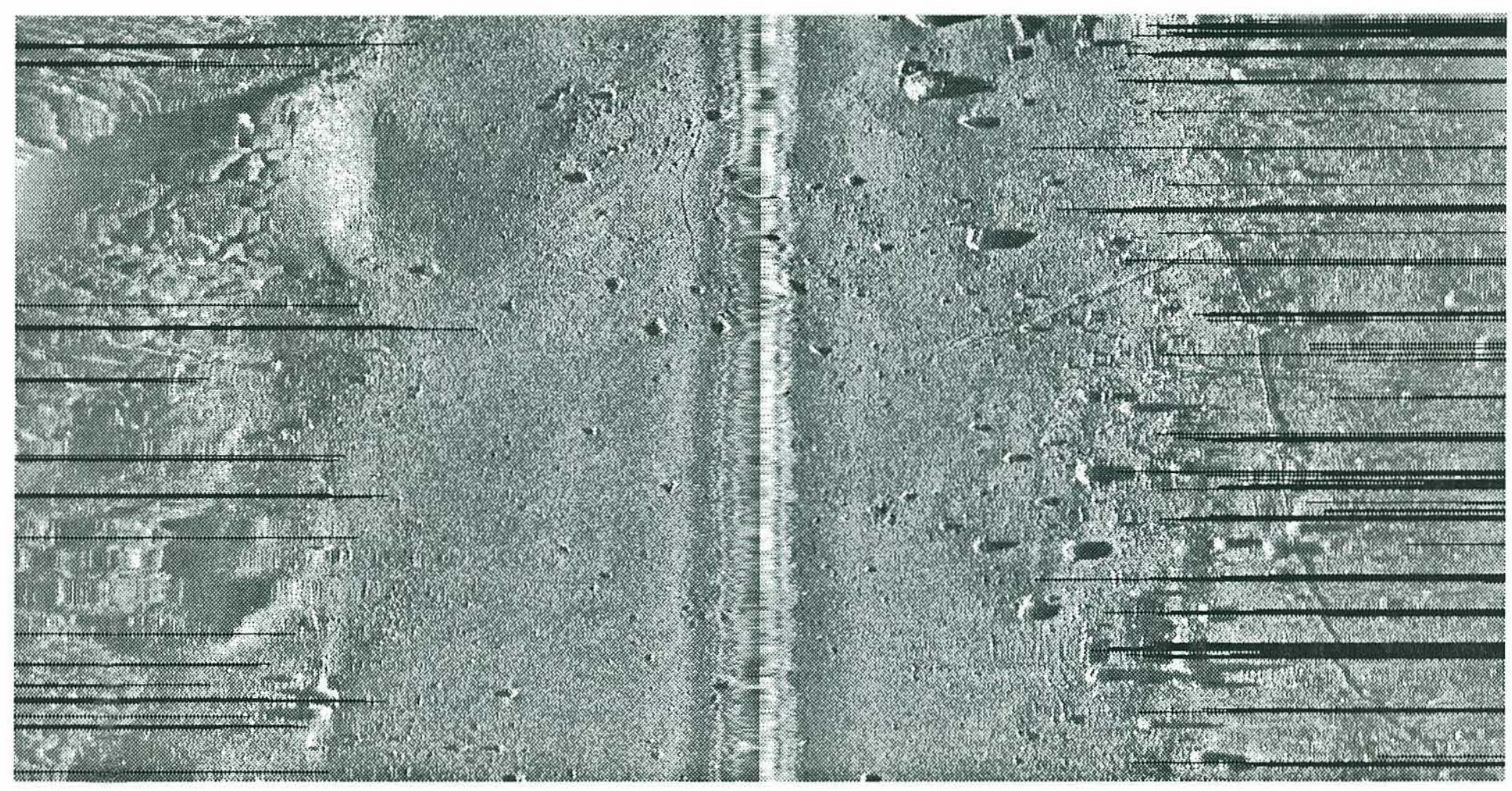

Figure 5.18: Sonograph of Fig. 5.17 after correction of the slant-range distortion and backscanning detection. 


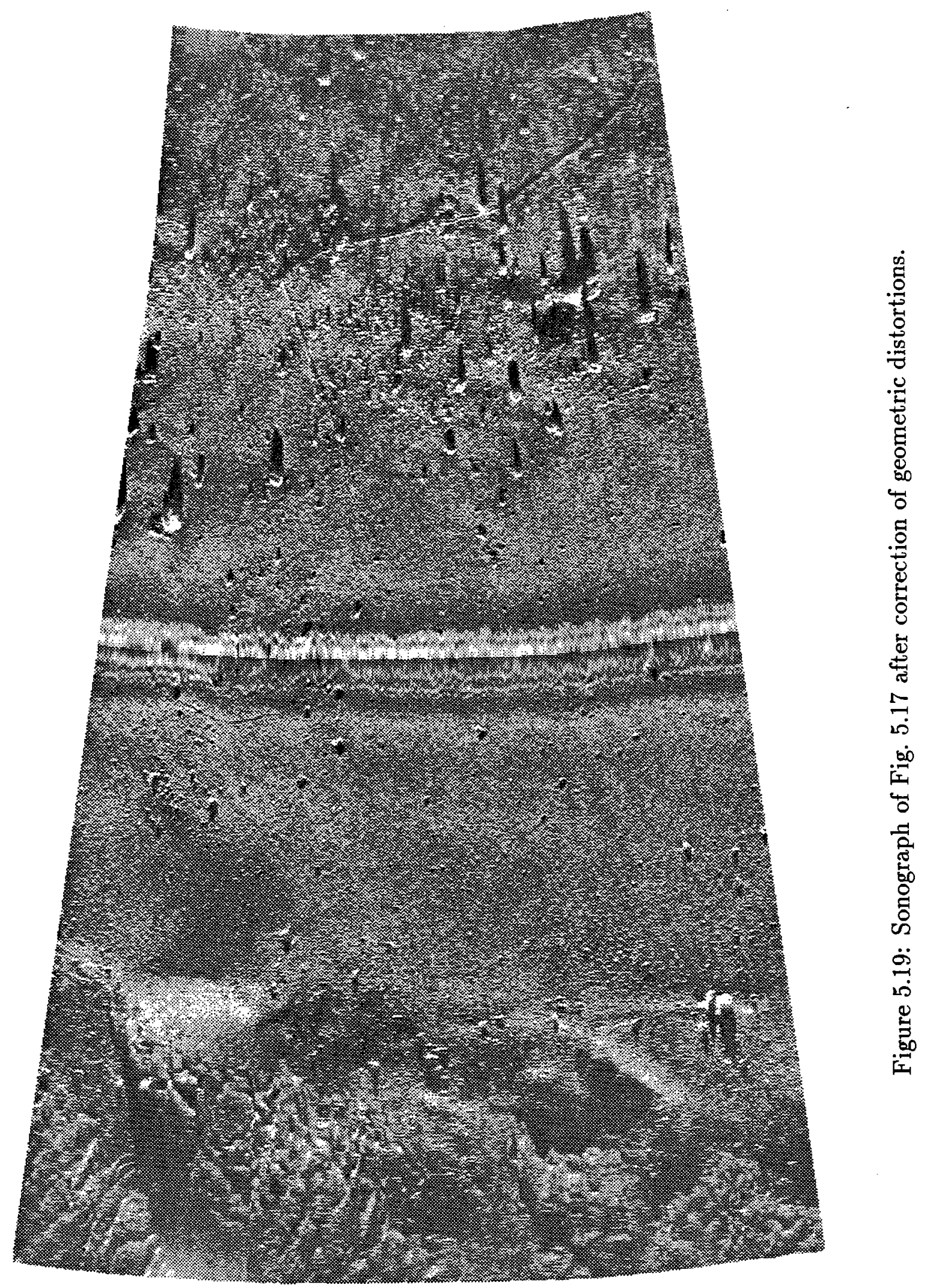



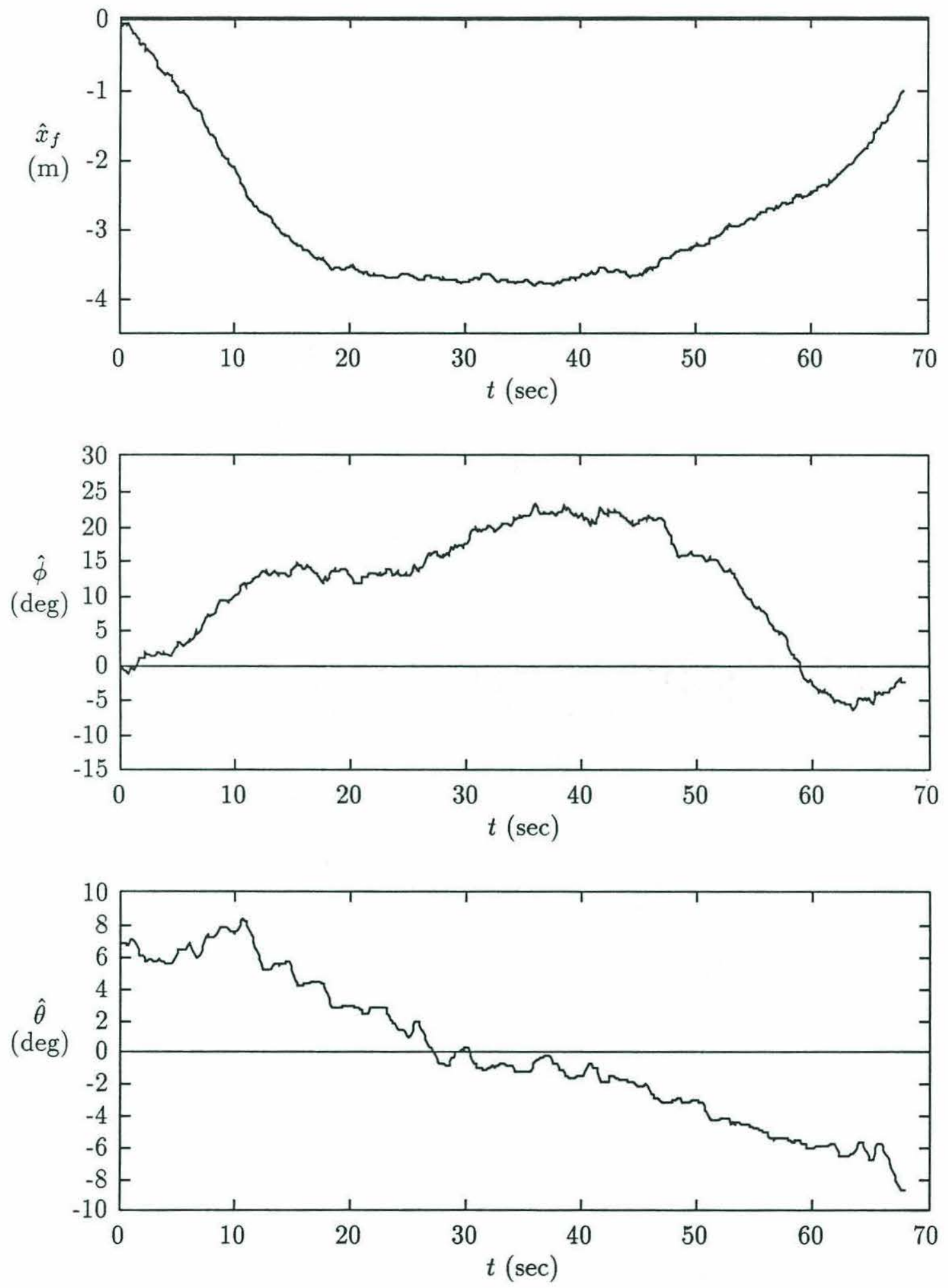

Figure 5.20: Estimates of attitude parameters for the sonograph of Fig. 5.17. 


\section{Chapter 6}

\section{Final Considerations}

In this chapter we offer final considerations concerning the theoretical developments, algorithms and results presented in this thesis, along with suggestions for future research on the correction of geometric distortions in sonographs.

\subsection{Evaluation of Results}

Visual inspection of the corrected sonographs presented in Chapter 5 offers strong indication that the techniques developed in this thesis can effectively reduce the degree of geometric distortion in sonographs, thus fulfilling their main goal. The algorithm is original in that it does not require navigational or attitude measurements for correcting the geometric distortions, but relies solely on the information contained in the image itself, and on certain assumptions about its statistical properties. In the process of correcting the geometric distortions, the algorithm produces other relevant information that can prove useful in the interpretation of sonographs. For instance, it provides a means of detecting lines affected by backscanning, apparently with relatively low percentages of false alarms and misses, as seen in Chapter 3. It also allows reconstruction of the sampling pattern on the bottom in the form of mesh plots such as the one presented in Chapter 5 .

Another by-product of the algorithm are estimates of the towfish attitude parameters, namely its lateral displacement in the cross-track direction and its pitch and yaw angles. Because of the accumulation of the estimation errors of parameter increments, 
the estimates of attitude parameters become progressively less reliable, as indicated by the widening of the confidence intervals shown in the plots of Chapter 4, and, consequently, so do the estimates of sampling point coordinates. The estimates of distortion parameters, $\Delta x_{o}^{\prime}[n], \Delta y_{o}^{\prime}[n]$ and $\Delta \theta[n]$, however, remain just as accurate, which means that the relative location of points of the image with respect to neighboring points can be estimated more accurately than their absolute location. In other words, the algorithm is more capable of correcting local geometric distortion than of determining the absolute location of objects in the image.

The simulation carried out in Chapter 5 provides more objective evidence of the efficacy of the different forms of the algorithm. According to that simulation, very accurate estimates of the towfish yaw angle are obtained through both deterministic least-squares estimation and adaptive Kalman filtering, as well as fairly accurate estimates of its horizontal lateral displacement. The estimates of pitch are less reliable, because variations in pitch angle produce less geometric distortion than equal variations in yaw angle.

The estimates of distortion and attitude parameters obtained through the adaptive Kalman filter do not differ considerably from those obtained through deterministic leastsquares estimation, because all the parameters of the state-space model used in the filter are unknown and are simultaneously estimated with the distortion parameters. In that case, the state-space model contributes little a priori information. Improved results can be expected if the state-space model is derived from a theoretical analysis of the dynamics of the towfish and only a few of its parameters are unknown.

\subsection{Suggestions for Future Research}

The most important path for future research on the technique presented in this thesis is a more definitive evaluation of its accuracy. One way that may be accomplished is through further and more extensive simulations. Definitive evaluation, however, can be accomplished by comparing the estimates of attitude parameters with real measurements obtained through attitude sensors mounted on a towfish. Another possible means of evaluation is to acquire sonographs of areas for which sufficiently detailed bathymetric 
charts are available, and then attempt to match points in the corrected sonographs with points in the chart.

Another goal for future research could be to make the estimates of distortion parameters more robust by improving the process of selection of observation points for each line of the sonograph. As mentioned in Chapter 3, that would probably require the inclusion of image segmentation techniques to divide the sonograph in areas where the correlation lengths may be assumed constant. A more straightforward modification that can also be made to the algorithm is to incorporate a high-pass filter or some other device to periodically correct the estimates of attitude parameters in order to keep them from diverging as a result of the accumulation of the estimation errors of the parameter increments.

As pointed out in Chapter 4, our intent in employing a Kalman filter in the estimation of distortion parameters is to provide a framework in which knowledge of the dynamics of the towfish may be readily incorporated in the future. Improvements in the estimates can in principle be obtained by replacing the general auto-regressive model we adopted with a more specific state-space model derived from a mechanical analysis of the dynamics of towed cylinders. Additional improvements can be expected if the estimation of distortion parameters is carried out through smoothing or fixed-lag smoothing instead of through regular Kalman filtering, and if a greater number of observation points is used.

On a more practical side, an issue to be investigated is the feasibility of implementing the algorithm in real time, so that the corrected image may be seen on a display in the deploying vessel as the towfish scans the bottom.

\subsection{Conclusions}

The main contribution of this thesis is the development of a technique for the estimation and correction of geometric distortions in side-scan sonar images. Other techniques previously reported in the literature utilize navigational data to correct large-scale distortions caused by variations in the course of the deploying vessel and by the slant-range effect. In the case of a relatively few more sophisticated units equipped with attitude sensors, it is also possible to correct geometric distortions due to motion instabilities of the 
towfish. By comparison, in the technique presented in this thesis the geometric distortion is estimated from the image itself, requiring no navigational or attitude measurements. It may thus be applied to any existing digitized sonographs or to analog sonographs stored on magnetic tape.

A very detailed analysis of the side-scan sonar geometry was carried out, leading to a nonlinear model that expresses the sampling point coordinates as a function of the towfish attitude parameters. Unlike previous studies found in the side-scan sonar literature (see Chapter 2), this model takes into account the misalignment of the transmitting and receiving beams.

The techniques presented here are likely to find wider application in the enhancement of high-resolution sonographs (roughly, those with operating frequencies of $100 \mathrm{kHz}$ or higher). High-resolution side-scan sonars are usually very compact, portable units in which the transducer array is mounted on a relatively small towfish. In this type of sonar, the towfish is typically not equipped with sensors to provide attitude measurements that could be used for correcting geometric distortions. Furthermore, their small size makes them more susceptible to motion instabilities than their larger and heavier long-range counterparts. Those factors make this type of side-scan sonar the most likely beneficiary of the techniques presented here. 


\section{Appendix A}

\section{Summary of Notation}

The tables presented in this Appendix list the definitions of variables by chapter, in order of appearance. For the complete definition of each variable, refer to the sections, equations or figures listed in the reference columns.

Table A.1: Definitions from Chapter 2

\begin{tabular}{|c|l|l|}
\hline Symbol & \multicolumn{1}{|c|}{ Description } & \multicolumn{1}{c|}{ Reference } \\
\hline \hline$b$ & Backscattering strength on the seabed. & Sec. 2.1 \\
$s$ & Image intensity of the digitized sonograph. & Sec. 2.1 \\
{$[m, n]$} & Coordinate system defined on the sonograph. & Fig. 2.1 \\
$(x, y, z)$ & Coordinate system defined on the seabed. & Fig. 2.1 \\
$\left(x_{s}, y_{s}\right)$ & Coordinates of sampling points. & Sec. 2.1, Fig. 2.4 \\
$\left(x_{f}, y_{f}, z_{f}\right)$ & Coordinates of the towfish. & Fig. 2.3 \\
$\phi$ & Pitch angle of the towfish. & Figs. 1.4, 2.3 \\
$\theta$ & Yaw angle of the towfish. & Figs. 1.4, 2.3 \\
$t$ & Time from start of acquisition of the sonograph. & Sec. 2.2.1 \\
$t_{T}$ & Time when a pulse is transmitted. & Sec. 2.2.1 \\
$t_{R}$ & Time when the returned signal is sampled. & Sec. 2.2.1 \\
$\left(x_{e}, y_{e}, z_{e}\right)$ & Point on the axial plane of the effective beam. & Eq. $(2.3)$, Fig. 2.4 \\
$\phi_{e}, \theta_{e}$ & Pitch and yaw angle of the effective beam. & Eq. 2.2$),$ Fig. 2.4 \\
$\left(x_{o}, y_{o}\right)$ & Reference point in the effective beam plane. & Fig. 2.4 \\
$d$ & Distance between $\left(x_{s}, y_{s}\right)$ and ( $\left.x_{o}, y_{o}\right)$. & Fig.2.3 \\
$h$ & Distance between $\left(x_{o}, y_{o}\right)$ and the towfish. & Fig. 2.3 \\
$r$ & Range from a sampling point to the towfish. & Sec. 2.2.1 \\
$c$ & Speed of sound in water. & Sec. 2.2.1 \\
\hline
\end{tabular}


Table A.2: Definitions from Chapter 2 (Continued)

\begin{tabular}{|c|l|l|}
\hline Symbol & \multicolumn{1}{|c|}{ Description } & \multicolumn{1}{c|}{ Reference } \\
\hline \hline$T_{f}$ & Firing period of the sonar. & Sec. 2.2 .1 \\
$T_{s}$ & Sampling period used in digitizing the signal. & Sec. 2.2 .1 \\
$N_{m}$ & Number of samples per line of the sonograph. & Sec. 2.2 .1 \\
$N_{n}$ & Number of lines in the sonograph. & Sec. 2.2 .1 \\
$\bar{s}$ & Image after correction of the slant-range distortion. & Sec. 2.2 .2 \\
$N_{l}$ & Number of pixels per line per channel of $\bar{s}$. & Sec. 2.2 .2 \\
$\Delta_{n} x_{s}$ & Sampling displacement in the $x$ direction. & Eq. (2.7a), Fig. 2.5 \\
$\Delta_{n} y_{s}$ & Sampling displacement in the $y$ direction. & Eq. (2.7b), Fig. 2.5 \\
$\left(x^{\prime}, y^{\prime}\right)$ & Auxiliary coordinate system. & Fig. 2.6 \\
\hline
\end{tabular}

Table A.3: Definitions from Chapter 3

\begin{tabular}{|c|l|l|}
\hline Symbol & \multicolumn{1}{|c|}{ Description } & \multicolumn{1}{|c|}{ Reference } \\
\hline \hline$R_{b}$ & Autocorrelation function of $b(x, y)$. & Sec. 3.1 \\
$s_{u}$ & Upsampled sonograph image. & Sec. 3.1 \\
$N_{u}$ & Upsampling factor. & Sec. 3.1 \\
$l^{\prime}$ & Cross-track coordinate in the upsampled image. & Sec. 3.1 \\
$n^{\prime}$ & Along-track coordinate in the upsampled image. & Sec. 3.1 \\
$\mu_{s}$ & Mean of line segments. & Eq. $(3.1)$ \\
$\sigma_{s}$ & Standard deviation of line segments. & Eq. $(3.2)$ \\
$\rho$ & Normalized cross-correlation of line segments. & Eq. 3.3. Fig. 3.1 \\
$\Delta l^{\prime}$ & Relative shift between line segments in the $l^{\prime}$ direction. & Fig. 3.1 \\
$\Delta n^{\prime}$ & Relative shift between line segments in the $n^{\prime}$ direction. & Fig. 3.1 \\
$2 L+1$ & Length of line segments for calculating cross-correlations. & Fig. 3.1 \\
$P_{n}$ & Sampling interval in the along-track direction. & Fig. 3.9 \\
$L_{n}^{+}, L_{n}^{-}$ & Positive- and negative-lag correlation lengths. & Fig. 3.7 \\
$\rho_{o}$ & Threshold for calculating the correlation length. & Fig. 3.7 \\
$L_{b}$ & Correlation length of the backscattering function $b(x, y)$. & Sec. 3.3 .1 \\
$L_{n o}$ & Average correlation length in the absence of distortions. & Sec. 3.3 .1 \\
$q$ & Scaled sampling displacements. & Eq. $(3.9)$ \\
$D$ & Matrix of coefficients for the system of equations on $q$. & Sec. 3.3 .1 \\
$\underline{b}$ & Inhomogeneous vector for the system of equations on $q$. & Eq. $(3.11)$ \\
$\mathcal{N}$ & Set of lines chosen for estimation of $L_{n o}$. & Sec. 3.8 \\
$N_{\mathcal{N}}$ & Number of lines in $\mathcal{N}$. & Sec. 3.8 \\
$v$ & Average speed of the deploying vessel. & Sec. 3.8 \\
$v_{\mathcal{N}}$ & Average speed of the towfish over the lines in $\mathcal{N}$. & Sec. 3.8 \\
\hline
\end{tabular}


Table A.4: Definitions from Chapter 4

\begin{tabular}{|c|c|c|}
\hline Symbol & Description & Reference \\
\hline$\overline{\mathcal{L}}$ & Set of distances of selected observation points. & Sec. 4.1 \\
\hline$p$ & Number of points in $\mathcal{L}$ & Sec. 4.1 \\
\hline$\varepsilon_{x}^{2}, \varepsilon_{y}^{2}$ & Chi-square cost functions. & Sec. 4.2 \\
\hline$\sigma_{\overparen{\Delta x^{\prime}}}^{2}$ & Variance of the estimation error of $\Delta x_{o}^{\prime}$ & Sec. 4.2 \\
\hline$\sigma_{\triangle y_{o}^{\prime}}^{2}$ & Variance of the estimation error of $\Delta y_{o}^{\prime}$ & Sec. 4.2 \\
\hline$\sigma_{\widehat{\Delta A}}^{2}$ & Variance of the estimation error of $\Delta \theta$ & Sec. 4.2 \\
\hline$\sigma_{\hat{x}_{0}}^{2}$ & Variance of the estimation error of $x_{0}$ & Sec. 4.2 \\
\hline$\sigma_{\hat{y}_{o}}^{2}$ & Variance of the estimation error of $y_{0}$ & Sec. 4.2 \\
\hline$\sigma_{\hat{\theta}}^{2}$ & Variance of the estimation error of $\theta$ & Sec. 4.2 \\
\hline$\underline{\zeta}$ & Observation vector. & Eq. (4.13) \\
\hline$\underline{\xi}$ & Vector of distortion parameters. & Eq. (4.13) \\
\hline$\underline{v}$ & Observation noise vector. & Eq. (4.13) \\
\hline$C$ & Observation matrix. & Eq. (4.14) \\
\hline$\Lambda_{\zeta}, \Lambda_{\xi}$ & Covariance matrices of $\underline{\zeta}$ and $\xi$. & Sec. 4.3 .1 \\
\hline$\Lambda_{\xi \zeta}, \Lambda_{\xi v}$ & Cross-covariance matrices of $\underline{\xi}$ with $\underline{\zeta}$ and $\underline{v}$. & Sec. 4.3.1 \\
\hline$N$ & Order of the state-space model. & Sec. 4.3 .2 \\
\hline$\Xi$ & State vector. & Sec. 4.3 .2 \\
\hline A & State transition matrix. & Eq. (4.16) \\
\hline$B$ & Input matrix. & Eq. (4.16) \\
\hline $\bar{C}$ & Redefined observation matrix. & Eq. (4.16) \\
\hline$\underline{w}$ & Process noise vector. & Eq. (4.16) \\
\hline$R$ & Covariance matrix of $\underline{w}$. & Sec. 4.3 .2 \\
\hline$S$ & Cross-covariance matrix of $\underline{v}$ and $\underline{w}$. & Sec. 4.3 .2 \\
\hline$\underline{a}$ & Auxiliary vector of the information filter. & Sec. 4.3 .3 \\
\hline$P$ & Covariance matrix of the state estimation error. & Sec. 4.3 .3 \\
\hline$G, N$ & Auxiliary matrices in the information filter. & Sec. 4.3 .3 \\
\hline$\beta$ & Forgetting factor. & Secs. $4.3 .3,4.3 .4$ \\
\hline$A_{1}, \ldots, A_{N}$ & Matrices of the multi-dimensional AR model. & Sec. 4.3 .4 \\
\hline$\underline{\alpha}$ & Vector of model parameters. & Sec. 4.3.4 \\
\hline$\chi$ & Regression matrix. & Sec. 4.3.4 \\
\hline$U$ & Covariance matrix of the parameter estimation error. & Sec. 4.3.4 \\
\hline$J$ & Auxiliary matrix in the RLS algorithm. & Sec. 4.3.4 \\
\hline
\end{tabular}


Table A.5: Definitions from Chapter 5

\begin{tabular}{|c|l|l|}
\hline Symbol & \multicolumn{1}{|c|}{ Description } & Reference \\
\hline \hline$s_{c}$ & Image after correction in the cross-track direction. & Sec. 5.2.1 \\
$l^{\prime}$ & Cross-track coordinate of $s_{c}$. & Sec. 5.2.1 \\
$N_{l^{\prime}}$ & Number of pixels per line per channel in $s_{c}$. & Sec. 5.2.1 \\
$l_{c}$ & Coordinates for resampling the lines of $\bar{s}$. & Sec. 5.2 .1 \\
$\left(x_{c}, y_{c}\right)$ & Coordinates of sampling points associated with $s_{c}$. & Sec. 5.2 .1 \\
$S_{n}$ & DFT of the $n$-th line of $\bar{s}$ & Sec. 5.2.1 \\
$s_{a}$ & Final image after correction of geometric distortions. & Sec. 5.2.2 \\
$n^{\prime}$ & Along-track coordinate of $s_{a}$. & Sec. 5.2.2 \\
$N_{n^{\prime}}$ & Number of pixels per column in $s_{a}$. & Sec. 5.2.2 \\
$n_{a}$ & Coordinates for resampling the columns of $s_{c}$. & Sec. 5.2.2 \\
$S_{l^{\prime}}$ & DFT of the $l^{\prime}$-th line of $s_{c}$ & Sec. 5.2.2 \\
\hline
\end{tabular}




\section{Appendix B}

\section{Derivation of Results from the Geometry of the Problem}

In Section 2.2.1 it was necessary to express the distance $d$ defined in Fig. 2.4 as a function of the towfish attitude parameters at times $t_{T}$ and $t_{R}$. The key to deriving this expression is that the round-trip travel time of the wavefront from the point where the towfish was located at time $t_{T}$ to the sampling point $\left(x_{s}\left(t_{R}\right), y_{s}\left(t_{R}\right)\right)$ and back to the point where the towfish was located at time $t_{R}$ has to equal $\left(t_{R}-t_{T}\right)$. Let us denote by $r_{T}$ and $r_{R}$ the distances from points $\left(x_{f}\left(t_{T}\right), y_{f}\left(t_{T}\right), z_{f}\left(t_{T}\right)\right)$ and $\left(x_{f}\left(t_{R}\right), y_{f}\left(t_{R}\right), z_{f}\left(t_{R}\right)\right)$ to point $\left(x_{s}\left(t_{R}\right), y_{s}\left(t_{R}\right)\right)$, i. e.,

$$
\begin{aligned}
& r_{T} \triangleq\left(\left[x_{f}\left(t_{T}\right)-x_{s}\left(t_{R}\right)\right]^{2}+\left[y_{f}\left(t_{T}\right)-y_{s}\left(t_{R}\right)\right]^{2}+z_{f}^{2}\left(t_{T}\right)\right)^{\frac{1}{2}} \\
& r_{R} \triangleq\left(\left[x_{f}\left(t_{R}\right)-x_{s}\left(t_{R}\right)\right]^{2}+\left[y_{f}\left(t_{R}\right)-y_{s}\left(t_{R}\right)\right]^{2}+z_{f}^{2}\left(t_{R}\right)\right)^{\frac{1}{2}} .
\end{aligned}
$$

Then, as argued above, we must have

$$
r_{T}+r_{R}=c\left(t_{R}-t_{T}\right)
$$

where $c$ is the speed of sound in water. This equation describes a three-dimensional ellipsoid with focii at points $\left(x_{f}\left(t_{T}\right), y_{f}\left(t_{T}\right), z_{f}\left(t_{T}\right)\right)$ and $\left(x_{f}\left(t_{R}\right), y_{f}\left(t_{R}\right), z_{f}\left(t_{R}\right)\right)$. We will denote by $r$ the average of $r_{T}$ and $r_{R}$,

$$
r \triangleq \frac{r_{T}+r_{R}}{2}
$$


Thus, $r$ is also given by

$$
r=\frac{c\left(t_{R}-t_{T}\right)}{2} .
$$

From Fig. 2.4 we had obtained Eqs. (2.4a) and (2.4b), repeated here for convenience

$$
\begin{aligned}
& x_{s}\left(t_{R}\right)=x_{o}\left(t_{R}\right)+d \cos \theta_{e}\left(t_{R}\right) \\
& y_{s}\left(t_{R}\right)=y_{o}\left(t_{R}\right)+d \sin \theta_{e}\left(t_{R}\right) .
\end{aligned}
$$

Our task now is to solve Eqs. (B.1)-(B.5) for $d$, eliminating $x_{s}\left(t_{R}\right)$ and $y_{s}\left(t_{R}\right)$. The solution will determine the two points where the three dimensional ellipsoid is intersected by the line defined by the intersection of the axial plane of the effective beam and the seabed plane.

From Eq. (B.3) we have

$$
\begin{aligned}
r_{T}^{2}-r_{R}^{2}= & {\left[x_{f}\left(t_{T}\right)-x_{s}\left(t_{R}\right)\right]^{2}+\left[y_{f}\left(t_{T}\right)-y_{s}\left(t_{R}\right)\right]^{2}+z_{f}^{2}\left(t_{T}\right) } \\
& -\left[x_{f}\left(t_{R}\right)-x_{s}\left(t_{R}\right)\right]^{2}-\left[y_{f}\left(t_{R}\right)-y_{s}\left(t_{R}\right)\right]^{2}-z_{f}^{2}\left(t_{R}\right) \\
= & {\left[x_{f}\left(t_{T}\right)-x_{o}\left(t_{R}\right)-d \cos \theta_{e}\left(t_{R}\right)\right]^{2} } \\
& +\left[y_{f}\left(t_{T}\right)-y_{o}\left(t_{R}\right)-d \sin \theta_{e}\left(t_{R}\right)\right]^{2}+z_{f}^{2}\left(t_{T}\right) \\
& -\left[x_{f}\left(t_{R}\right)-x_{o}\left(t_{R}\right)-d \cos \theta_{e}\left(t_{R}\right)\right]^{2} \\
& -\left[y_{f}\left(t_{R}\right)-y_{o}\left(t_{R}\right)-d \sin \theta_{e}\left(t_{R}\right)\right]^{2}-z_{f}^{2}\left(t_{R}\right) \\
= & {\left[x_{f}\left(t_{T}\right)-x_{o}\left(t_{R}\right)\right]^{2}+\left[y_{f}\left(t_{T}\right)-y_{o}\left(t_{R}\right)\right]^{2}+z_{f}^{2}\left(t_{T}\right) } \\
& +2 d \cos \theta_{e}\left(t_{R}\right)\left[x_{f}\left(t_{R}\right)-x_{f}\left(t_{T}\right)\right] \\
& -\left[x_{f}\left(t_{R}\right)-x_{o}\left(t_{R}\right)\right]^{2}-\left[y_{f}\left(t_{R}\right)-y_{o}\left(t_{R}\right)\right]^{2}-z_{f}^{2}\left(t_{R}\right) \\
& +2 d \sin \theta_{e}\left(t_{R}\right)\left[y_{f}\left(t_{R}\right)-y_{f}\left(t_{T}\right)\right],
\end{aligned}
$$

or

$$
r_{T}^{2}-r_{R}^{2}=2 e d+f,
$$

where

$$
\begin{aligned}
e \triangleq & {\left[x_{f}\left(t_{R}\right)-x_{f}\left(t_{T}\right)\right] \cos \theta_{e}\left(t_{R}\right)-\left[y_{f}\left(t_{R}\right)+y_{f}\left(t_{T}\right)\right] \sin \theta_{e}\left(t_{R}\right) } \\
f \triangleq & {\left[x_{f}\left(t_{T}\right)-x_{o}\left(t_{R}\right)\right]^{2}+\left[y_{f}\left(t_{T}\right)-y_{o}\left(t_{R}\right)\right]^{2}+z_{f}^{2}\left(t_{T}\right) } \\
& -\left[x_{f}\left(t_{R}\right)-x_{o}\left(t_{R}\right)\right]^{2}-\left[y_{f}\left(t_{R}\right)-y_{o}\left(t_{R}\right)\right]^{2}-z_{f}^{2}\left(t_{R}\right) .
\end{aligned}
$$


From Eqs. (B.1), (B.2), (B.4), and (B.5) we also have

$$
\begin{aligned}
& r_{T}^{2}+r_{R}^{2}+2 r_{T} r_{R}=4 r^{2} \\
\Rightarrow & 2 r_{T} r_{R}=4 r^{2}-r_{T}^{2}-r_{R}^{2} \\
\Rightarrow & 4 r_{T}^{2} r_{R}^{2}=16 r^{4}+r_{T}^{4}+r_{R}^{4}+2 r_{T}^{2} r_{R}^{2}-8 r^{2}\left(r_{T}^{2}+r_{R}^{2}\right) \\
\Rightarrow & 16 r^{4}-8 r^{2}\left(r_{T}^{2}+r_{R}^{2}\right)+r_{T}^{4}+r_{R}^{4}-2 r_{T}^{2} r_{R}^{2}=0 .
\end{aligned}
$$

Therefore,

$$
16 r^{4}-8 r^{2}\left(r_{T}^{2}+r_{R}^{2}\right)+\left(r_{T}^{2}-r_{R}^{2}\right)^{2}=0
$$

From Eqs. (B.1), (B.2), (B.4), and (B.5),

$$
\begin{aligned}
r_{T}^{2}+r_{R}^{2}= & {\left[x_{f}\left(t_{T}\right)-x_{s}\left(t_{R}\right)\right]^{2}+\left[y_{f}\left(t_{T}\right)-y_{s}\left(t_{R}\right)\right]^{2}+z_{f}^{2}\left(t_{T}\right) } \\
& +\left[x_{f}\left(t_{R}\right)-x_{s}\left(t_{R}\right)\right]^{2}+\left[y_{f}\left(t_{R}\right)-y_{s}\left(t_{R}\right)\right]^{2}+z_{f}^{2}\left(t_{R}\right) \\
= & {\left[x_{f}\left(t_{T}\right)-x_{o}\left(t_{R}\right)-d \cos \theta_{e}\left(t_{R}\right)\right]^{2} } \\
& +\left[y_{f}\left(t_{T}\right)-y_{o}\left(t_{R}\right)-d \sin \theta_{e}\left(t_{R}\right)\right]^{2}+z_{f}^{2}\left(t_{T}\right) \\
& +\left[x_{f}\left(t_{R}\right)-x_{o}\left(t_{R}\right)-d \cos \theta_{e}\left(t_{R}\right)\right]^{2} \\
& +\left[y_{f}\left(t_{R}\right)-y_{o}\left(t_{R}\right)-d \sin \theta_{e}\left(t_{R}\right)\right]^{2}+z_{f}^{2}\left(t_{R}\right) \\
= & {\left[x_{f}\left(t_{T}\right)-x_{o}\left(t_{R}\right)\right]^{2}+\left[y_{f}\left(t_{T}\right)-y_{o}\left(t_{R}\right)\right]^{2}+z_{f}^{2}\left(t_{T}\right) } \\
& +d^{2}-2 d \cos \theta_{e}\left(t_{R}\right)\left[x_{f}\left(t_{R}\right)+x_{f}\left(t_{T}\right)-2 x_{o}\left(t_{R}\right)\right] \\
& +\left[x_{f}\left(t_{R}\right)-x_{o}\left(t_{R}\right)\right]^{2}+\left[y_{f}\left(t_{R}\right)-y_{o}\left(t_{T}\right)\right]^{2}+z_{f}^{2}\left(t_{R}\right) \\
& +d^{2}-2 d \sin \theta_{e}\left(t_{R}\right)\left[y_{f}\left(t_{T}\right)+y_{f}\left(t_{R}\right)-2 y_{o}\left(t_{R}\right)\right],
\end{aligned}
$$

or

$$
r_{T}^{2}+r_{R}^{2}=2 d^{2}-2 g d+2 h^{2},
$$

where

$$
\begin{aligned}
g \triangleq & {\left[x_{f}\left(t_{R}\right)+x_{f}\left(t_{T}\right)-2 x_{o}\left(t_{R}\right)\right] \cos \theta_{e}\left(t_{R}\right)+\left[y_{f}\left(t_{R}\right)+y_{f}\left(t_{T}\right)-2 y_{o}\left(t_{R}\right)\right] \sin \theta_{e}\left(t_{R}\right) } \\
h^{2} \triangleq & \frac{1}{2}\left(\left[x_{f}\left(t_{T}\right)-x_{o}\left(t_{R}\right)\right]^{2}+\left[y_{f}\left(t_{T}\right)-y_{o}\left(t_{R}\right)\right]^{2}+z_{f}^{2}\left(t_{T}\right)\right. \\
& \left.+\left[x_{f}\left(t_{R}\right)-x_{o}\left(t_{R}\right)\right]^{2}+\left[y_{f}\left(t_{R}\right)-y_{o}\left(t_{R}\right)\right]^{2}+z_{f}^{2}\left(t_{R}\right)\right) .
\end{aligned}
$$


Substituting Eqs. (B.8) and (B.6) into (B.7) we obtain

$$
16 r^{4}-8 r^{2}\left(2 d^{2}-2 g d+2 h^{2}\right)+(2 e d+f)^{2}=0,
$$

which, after rearranging the terms, yields

$$
4\left(e^{2}-4 r^{2}\right) d^{2}+4\left(e f+4 g r^{2}\right) d+16 r^{4}-16 h^{2} r^{2}+f^{2}=0 .
$$

The solution to this quadratic equation in $d$ yields

$$
d=\frac{-4\left(e f+4 g r^{2}\right) \pm \sqrt{16\left(e f+4 g r^{2}\right)^{2}-16\left(e^{2}-4 r^{2}\right)\left(16 r^{4}-16 h^{2} r^{2}+f^{2}\right)}}{8\left(e^{2}-4 r^{2}\right)},
$$

and after simplification we obtain the final result

$$
d=\frac{e f-g r^{2} \pm 2 r \sqrt{16 r^{4}+4\left(g^{2}-e^{2}-4 h^{2}\right) r^{2}+f^{2}+2 e f g+4 e^{2} h^{2}}}{2\left(r^{2}-e^{2}\right)} .
$$




\section{Bibliography}

[1] Brian D. O. Anderson and John B. Moore. Optimal Filtering. Prentice-Hall, Inc., Englewood Cliffs, N. J., 1979.

[2] Jean-Marie Augustin. Side scan acoustic images processing software. In Proceedings of the 1986 Working Symposium on Oceanographic Data Systems, pages 221-228, San Diego, CA, February 1986.

[3] Jean-Marie Augustin and Michel Voisset. Images sonar et cartographie en géologie. L'Onde Électrique, 69(3):20-27, May/June 1989.

[4] J. M. Berkson and C. S. Clay. Transformation of side-scan sonar records to a linear display. Int. Hydrogr. Rev., 50(2):55-59, 1973.

[5] Phillip R. Bevington. Data Reduction and Error Analysis for the Physical Sciences. McGraw-Hill, New York, 1969.

[6] J. G. Blackinton, D. M. Hussong, and J. G. Kosalos. First results from a combination side-scan sonar and seafloor mapping system (SeaMARC II). In Proceedings of the 15th Annual Offshore Technology Conference, volume 1, pages 307-314, Houston, Texas, May 1983.

[7] R. S. Bryant. Side scan sonar for hydrography. Int. Hydrogr. Rev., 52(1):43-56, 1975.

[8] Pat S. Chavez, Jr. Processing techniques for digital sonar images from GLORIA. Photogrammetric Eng. and Remote Sens., 52(8):1133-1145, August 1986.

[9] W. D. Chesterman, P. R. Clynick, and A. H. Stride. An acoustic aid to seabed survey. Acoustica, 8:285-290, 1958.

[10] C. S. Clay, John Ess, and Irving Weisman. Lateral echo sounding of the ocean bottom on the continental rise. J. of Geophysical Research, 69(18):3823-3835, September 1964.

[11] C. S. Clay and H. Medwin. Acoustical Oceanography. John Wiley \& Sons, New York, 1977.

[12] E. Clerici. Evaluation of relative effectiveness of some image processing techniques applied to side-scan sonar data. In Proceedings of the US-Australia Workshop on 
Image Processing Techniques for Remote Sensing, p.ıges 1-10, Carberra, Australia, May 1978.

[13] Peter Clifford. Real time seafloor mapping. Sea Technology, 20:22-26, May 1979.

[14] R. Cooper. An analysis of the effect of vehicle yaw on gloria sonographs. Report 6, Institute of Oceanographic Sciences, Godalming, U.K., 1974.

[15] P. N. Denbigh. A bathymetric side-scan sonar. In Ultrasonics International 79 Conference Procedings, pages 321-326, May 1979.

[16] P. N. Denbigh. A review of rapid depth measuring techniques and the development of bathymetric side scan sonar. In W. G. A. Russell-Cargill, editor, Recent Developments in Side Scan Sonar Techniques, chapter 3, pages 47-79. Central Acoustics Laboratory, University of Cape Town, South Africa, 1982.

[17] P. N. Denbigh. Stereoscopic visualization and contour mapping of the sea bed using a bathymetric sidescan sonar (BASS). The Radio and Electronic Engineer, 53(7/8):301-307, July/August 1983.

[18] P. N. Denbigh and B. W. Flemming. Range prediction and calibration in side scan sonar. In W. G. A. Russell-Cargill, editor, Recent Developments in Side Scan Sonar Techniques, chapter 4, pages 81-100. Central Acoustics Laboratory, University of Cape Town, South Africa, 1982.

[19] T. D. Donovan and A. H. Stride. An acoustic survey of the sea floor south of Dorset and its geological interpretation. Philos. Trans. R. Soc., B244:299-330, 1961.

[20] C. Eaves-Walton and G. A. Shippey. Digital image processing for sidescan sonar data analysis. In Fifth International Conference on Electronics for Ocean Technology, number 72, pages 203-209, March 1987.

[21] H. E. Edgerton. Sonar Images. Prentice Hall, Englewood Cliffs, 1986.

[22] Marguerite Ennis and Johannes W. J. van Wick. Image processing on side scan sonar records. In Proceedings of the Fourth South African Symposium on Digital Image Processing, pages 83-93, Durban, South Africa, July 17-18 1986.

[23] B. W. Flemming. Causes and effects of sonograph distortion and some graphical methods for their manual correction. In W. G. A. Russell-Cargill, editor, Recent Developments in Side Scan Sonar Techniques, chapter 5, pages 103-138. Central Acoustics Laboratory, University of Cape Town, South Africa, 1982.

[24] B. W. Flemming. A historical introduction to underwater acoustics with special reference to echo sounding, sub-bottom profiling and side scan sonar. In W. G. A. Russell-Cargill, editor, Recent Developments in Side Scan Sonar Techniques, chapter 1, pages 3-9. Central Acoustics Laboratory, University of Cape Town, South Africa, 1982. 
[25] P. W. Flemming. Side-scan sonar: A practical guide. Int. Hydrogr. Rev., 53(1):65-92, January 1976.

[26] Arthur Gelb, editor. Applied Optimal Estimation. The MIT Press, Cambridge, Mass., 1974.

[27] Peter T. Gough. A synthetic aperture sonar system capable of operating at high speed and in turbulent media. IEEE J. Ocean Eng., OE-11(2):333-339, April 1986.

[28] J. C. Hopkins. A note on methods of producing corrected side-scan sonar displays. Int. Hydrogr. Rev., 49(2):100-106, 1972.

[29] J. C. Hopkins. Tape recording of side scanning sonar signals. Int. Hydrogr. Rev., 49(1):59-70, 1972.

[30] Didier Jan and Joël Minot. Les traitements d'image en sonar latéral. L'Onde Électrique, 69(3):13-19, May/June 1989.

[31] M. Klein. Side scan sonar. Undersea Technology, 8:24-26, April 1967.

[32] M. Klein. A modular sonar system for seabed mapping. In W. G. A. Russell-Cargill, editor, Recent Developments in Side Scan Sonar Techniques, chapter 2, pages 11-44. Central Acoustics Laboratory, University of Cape Town, South Africa, 1982.

[33] Dieter Kolouch. Interferometric side-scan sonar: A topographic sea-floor mapping system. Int. Hydrogr. Rev., 61(2):35-49, July 1984.

[34] Donald J. Krotser and Martin Klein. Side-scan sonar: Selective textural enhancement. In Oceans ' 76 Conference Proceedings, pages 16E-1 to 16E-6. IEEE-MTS, September 1976.

[35] W. Kunze. General aspects of application of horizontal echo sounding method to shipping. Int. Hydrogr. Rev., 34:63-72, 1957.

[36] A. S. Laughton. The first decade of GLORIA. J. of Geophysical Research, 86(B12):11511-11534, December 1981.

[37] O. Leenhardt. Side scanning sonar - a theoretical study. Int. Hydrogr. Rev., 51:6180, 1974.

[38] Lennart Ljung. System Identification. Prentice-Hall, Inc., Englewood Cliffs, N. J., 1987.

[39] Bruce P. Luyendyk, Earl J. Hajic, and David S. Simonett. Side-scan sonar mapping and computer-aided interpretation in the santa barbara channel, california. Marine Geophysical Researches, 5(4):365-388, 1983.

[40] Raman K. Mehra. On-line identification of linear dynamic systems with applications to kalman filtering. IEEE Trans. on Automatic Control, AC-16(1), February 1971. 
[41] Peter R. Paluzzi et al. Computer image processing in marine resource exploration. In Oceans '76 Conference Proceedings, pages 4D-1 to 4D-10, Washington, D.C., September 1976. IEEE-MTS.

[42] Peter R. Paluzzi et al. Computer rectification and mosaicking of side-looking sonar images. In Proceedings of the 13-th Annual Offshore Technology Conference, pages 103-114, Houston, Texas, May 1981.

[43] Athanasios Papoulis. Probability, Random Variables, and Stochastic Processes. McGraw-Hill, Inc., New York, $2^{\text {nd }}$ edition, 1984.

[44] J. M. Preston. Coordinates as determined by side scan sonar. Technical Memorandum 88-2, Defence Research Establishment Pacific, Victoria, BC, January 1988.

[45] David B. Prior, James M. Coleman, and Louis E. Garrison. Digitally acquired undistorted side scan sonar images of submarine landslides, Mississippi River delta. Geology, 7:423-425, September 1979.

[46] Thomas Beckett Reed IV and Donald Hussong. Digital image processing techniques for enhancement and classification of SeaMARC II side scan sonar imagery. J. of Geophysical Research, 94(B6):7469-7490, June 1989.

[47]. Stuart Rusby. A long range side-scan sonar for use in the deep sea (G.L.O.R.I.A. Project). Int. Hydrogr. Rev., 47(2):25-39, 1970.

[48] I. C. Russel. Dual channel sidescan sonar. Int. Hydrogr. Rev., 55(1):27-100, January 1978.

[49] Masaaki Shishido and Kenji Naito. A study on picture improvement for side looking sonar - part 1. NEC Research \& Development, (53):62-74, April 1979.

[50] Merrill Ivan Skolnik. Introduction to Radar Systems. McGraw-Hill, Inc., New York, $2^{\text {nd }}$ edition, 1980 .

[51] M. L. Somers et al. GLORIA II - an improved long range sidescan sonar. In Oceanology International Conference Proceedings, pages 16-24, London, 1978.

[52] M. L. Somers and A. R. Stubbs. Sidescan sonar. IEEE Proceedings, 131(3):243-256, June 1984.

[53] James Spottiswoode and Maxwell Dobson. A fibre optic recording oscilloscope for the display of side-scan sonar and seismic data. Marine Geology, 18(5):M73-M76, 1975 .

[54] T. K. Stanton. Sonar estimates of seafloor microroughness. J. Acoust. Soc. Am., 75(3):809-818, March 1984.

[55] W. Kenneth Stewart, Jr. Multisensor Modeling Underwater with Uncertain Information. PhD thesis, Massachusetts Institute of Technology, Cambridge, Mass., July 1988. 
[56] A. R. Stubbs. Identification of patterns on asdic records. Int. Hydrogr. Rev., 40(2):53-68, 1963.

[57] P. G. Teleki et al. Sonar survey of the U.S. atlantic continental slope; acoustic characteristics and image processing techniques. In Proceedings of the 13-th Annual Offshore Technology Conference, pages 91-102, Houston, Texas, May 1981.

[58] D. G. Tucker. Sideways looking sonar for marine geology. Geo-Marine Technology, 2(9):18-23, October 1966.

[59] M. J. Tucker and A. R. Stubbs. A narrow-beam echo-ranger for fishery and geological investigations. British J. Appl. Phys., 12:103-110, 1961.

[60] R. J. Urick. Principles of Underwater Sound for Engineers. McGraw-Hill, New York, 1967.

[61] C. D. T. Walker. Development of a ground speed corrected side scan sonar display system. Ultrasonics, 16:108-110, May 1978.

[62] E. Whipp and D. A. Horne. Digitising of side-scan sonar signals. Ultrasonics, 14(5):201-204, September 1976. 